Florida International University FIU Digital Commons

3-18-2014

\title{
Mechanisms Governing the Eyewall Replacement Cycle in Numerical Simulations of Tropical Cyclones
}

zhenduo zhu

thornpig@gmail.com

DOI: $10.25148 /$ etd.FI14040859

Follow this and additional works at: https://digitalcommons.fiu.edu/etd

Part of the Atmospheric Sciences Commons

\section{Recommended Citation}

zhu, zhenduo, "Mechanisms Governing the Eyewall Replacement Cycle in Numerical Simulations of Tropical Cyclones" (2014). FIU Electronic Theses and Dissertations. 1389.

https://digitalcommons.fiu.edu/etd/1389 


\section{FLORIDA INTERNATIONAL UNIVERSITY}

Miami, Florida

\section{MECHANISMS GOVERNING THE EYEWALL REPLACEMENT CYCLE IN NUMERICAL SIMULATIONS OF TROPICAL CYCLONES}

A dissertation submitted in partial fulfillment of the requirements for the degree of DOCTOR OF PHILOSOPHY

in

GEOSCIENCES

by

Zhenduo Zhu 
To: Dean Kenneth G. Furton

College of Arts and Sciences

This dissertation, written by Zhenduo Zhu, and entitled Mechanisms Governing the Eyewall Replacement Cycle in Numerical Simulations of Tropical Cyclones, having been approved in respect to style and intellectual content, is referred to you for judgment.

We have read this dissertation and recommend that it be approved.

$\begin{array}{r}\hline \text { Hugh Willoughby } \\ \hline \text { Haiyan Jiang } \\ \hline \text { Ming Zhao } \\ \hline \text { Frank Marks } \\ \hline \text { Ping Zhu, Major Professor }\end{array}$

Date of Defense: March 18, 2014

The dissertation of Zhenduo Zhu is approved.

$\begin{array}{r}\text { Dean Kenneth G. Furton } \\ \text { College of Arts and Sciences } \\ \hline \begin{array}{r}\text { Dean Lakshmi N. Reddi } \\ \text { University Graduate School }\end{array}\end{array}$

Florida International University, 2014 


\section{ACKNOWLEDGMENTS}

First and foremost, I owe a debt of gratitude to my advisor, Dr. Ping Zhu, who has offered me substantial invaluable support, guidance and insight throughout my time at FIU. I count myself very blessed to have had the opportunity to work with him. Great appreciation is also extended to my committee members, Dr. Hugh Willoughby, Dr. Frank Marks, Dr. Haiyan Jiang and Dr. Ming Zhao, who have taken their time to offer support and helpful suggestions throughout this research.

I would also thank Dr. David Nolan for guiding me to set up idealized TC simulations in WRF, Cen Gao for helpful discussions about WRF simulations, Jonathan Furst for correcting my writing in the dissertation proposal and Konstantinos Menelaou for sharing with me his collection of useful codes and models.

This dissertation would be incomplete without mentioning the support from my family and friends over these years. I would especially thank my wife for her constant encouragement and love during this arduous process.

Financial support of this research has been provided by the FIU Presidential Fellowship and the National Science Foundation under grant AGS-0847332. 


\title{
ABSTRACT OF THE DISSERTATION \\ MECHANISMS GOVERNING THE EYEWALL REPLACEMENT CYCLE IN \\ NUMERICAL SIMULATIONS OF TROPICAL CYCLONES
}

\author{
by
}

Zhenduo Zhu

Florida International University, 2014

Miami, Florida

Professor Ping Zhu, Major Professor

Eyewall replacement cycle (ERC) is frequently observed during the evolution of intensifying Tropical Cyclones (TCs). Although intensely studied in recent years, the underlying mechanisms of ERC are still poorly understood, and the forecast of ERC remains a great challenge. To advance our understanding of ERC and provide insights in improvement of numerical forecast of ERC, a series of numerical simulations is performed to investigate ERCs in TC-like vortices on a f-plane. The simulated ERCs possess key features similar to those observed in real TCs including the formation of a secondary tangential wind maximum associated with the outer eyewall. The SawyerEliassen equation and tangential momentum budget analyses are performed to diagnose the mechanisms underlying the secondary eyewall formation (SEF) and ERC. Our diagnoses reveal crucial roles of outer rainband heating in governing the formation and development of the secondary tangential wind maximum and demonstrate that the outer rainband convection must reach a critical strength relative to the eyewall before SEF and the subsequent ERC can occur. A positive feedback among low-level convection, acceleration of tangential winds in the boundary layer, and surface evaporation that leads 
to the development of ERC and a mechanism for the demise of inner eyewall that involves interaction between the transverse circulations induced by eyewall and outer rainband convection are proposed. The tangential momentum budget indicates that the net tendency of tangential wind is a small residual resultant from a large cancellation between tendencies induced by the resolved and sub-grid scale (SGS) processes. The large SGS contribution to the tangential wind budget explains different characteristics of ERC shown in previous numerical studies and poses a great challenge for a timely correct forecast of ERC. The sensitivity experiments show that ERCs are strongly subjected to model physics, vortex radial structure and background wind. The impact of model physics on ERC can be well understood with the interaction among eyewall/outer rainband heating, radilal inflow in the boundary layer, surface layer turbulent processes, and shallow convection in the moat. However, further investigations are needed to fully understand the exhibited sensitivities of ERC to vortex radial structure and background wind. 


\section{TABLE OF CONTENTS}

CHAPTER

PAGE

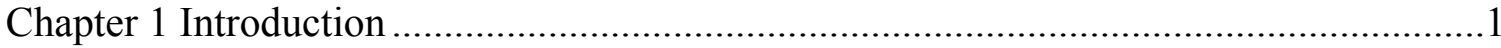

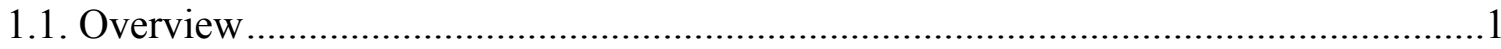

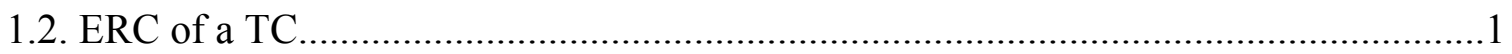

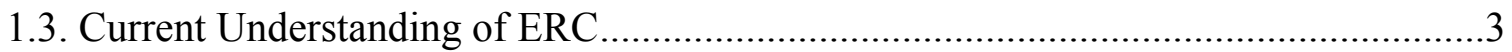

1.3.1. Secondary Eyewall Formation .........................................................

1.3.2. Demise of Primary Eyewall ……………………………………....

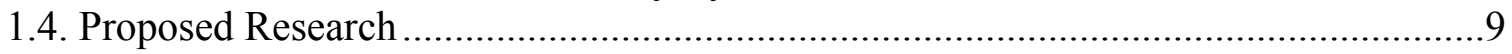

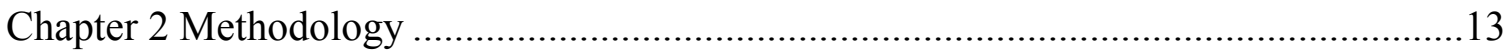

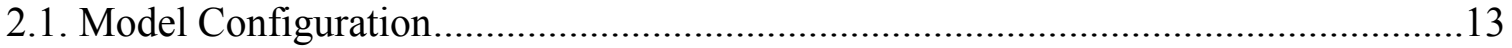

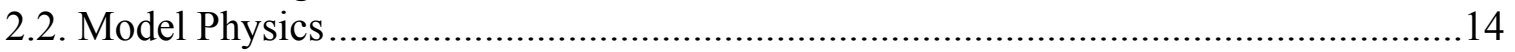

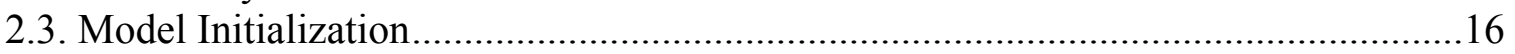

2.4. Cylindrical Coordinate Used for Analyses ...........................................................18

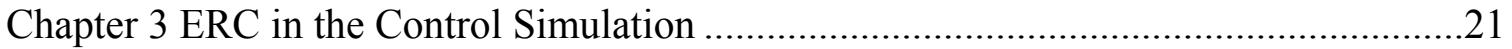

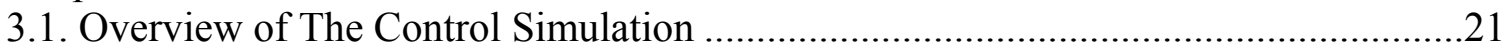

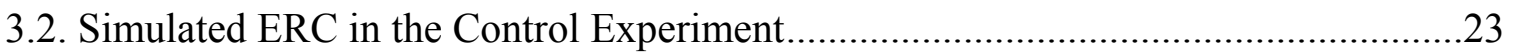

3.3. Axisymmetric Balanced Aspects of SEF and ERC ……….....................................27

3.3.1. Tangential Wind Budget and Sawyer-Eliassen Equation ........................................29

3.3.2. Validation of Sawyer-Eliassen Diagnoses ............................................................33

3.3.3. Formation and Development of Secondary Tangential Wind Maximum............35

3.3.4. Development of Outer Rainband Convection During ERC ....................................45

3.3.5. Interaction between Eyewall and Outer Rainbands During ERC .............................48

3.3.6. Propagation of Outer Rainband Convection During ERC ........................................51

Chapter 4 Sensitivities of ERC to Model Physics ……………………..........................82

4.1. Sensitivity of ERC to vertical SGS turbulent mixing parameterization ..........82

4.2. Complications of ERC in numerical simulations............................................97

4.2.1. Sensitivity of ERC to Initial Structure of Vortex ..............................98

4.2.2. Sensitivity of ERC to Snow Terminal Velocity ………...................101

4.2.3. Sensitivity of ERC to Radiation....................................................103

4.2.4. Impact of Background Wind on ERC ............................................104

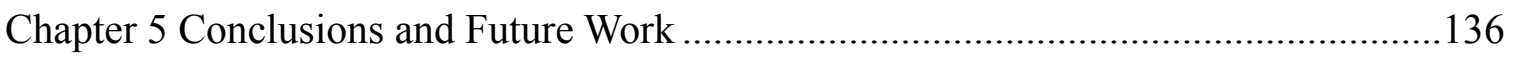

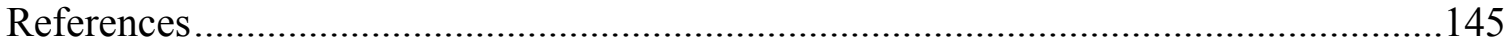

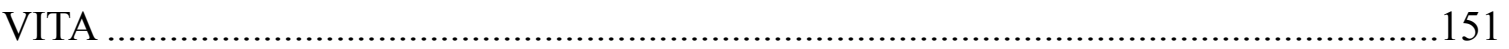




\section{LIST OF FIGURES}

FIGURE

PAGE

Figure 1.1 Research aircraft radar reflectivity composite of Ivan (2004) with concentric eyewalls.

Figure 2.1 Radial profiles of tangential wind (solid) and relative vorticity (dash) for the initial vortex used in the control simulation.

Figure 3.1 Time evolution of the minimum surface pressure (SLPMIN in hPa, black curve), the maximum mean tangential wind at $1 \mathrm{~km}$ (vtmax in $\mathrm{m} / \mathrm{s}$, blue curve), and the radius of maximum tangential wind (RMW in $\mathrm{km}$, green curve).

Figure 3.2 Snapshots of synthetic radar reflectivity (dBZ) at $1 \mathrm{~km}$ level from $56 \mathrm{~h}$ to $86 \mathrm{~h}$ at a time interval of 2 hours. The black circles indicate $50 \mathrm{~km}, 100$ $\mathrm{km}, 150 \mathrm{~km}$ and $200 \mathrm{~km}$ radii.

Figure 3.3 Radial profiles of azimuthal mean potential vorticity (smoothed with 5 passes of 1:2:1 smoother) at $1 \mathrm{~km}$ level between 66 and $78 \mathrm{~h}$ at a time interval of $6 \mathrm{~h}$.

Figure 3.4 Time-radius plots of azimuthal-mean (a) vertical velocity $(\mathrm{m} / \mathrm{s})$ at $5 \mathrm{~km}$ level, (b) tangential wind $(\mathrm{m} / \mathrm{s})$ at $1000 \mathrm{~m}$ level, (c) radial wind $(\mathrm{m} / \mathrm{s})$ at $500 \mathrm{~m}$ level. The contours in (c) are horizontal divergence at $500 \mathrm{~m}$ level, with negative and positive values plotted in white and black colors, respectively. Contour intervals are 10 for values between -50 . and 50., and 25 for values above 50 . and below -50 .

Figure 3.5 Azimuthal-mean vertical velocities simulated by WEF (left column) and those from the Sawyer-Eliassen diagnoses at the $65^{\text {th }} \mathrm{h}$ and $80^{\text {th }} \mathrm{h}$. Black contours in the figure indicate zero vertical velocities.

Figure 3.6 Correlation coefficient and the associated confidence level between the azimuthal-mean vertical velocities simulated by WRF and those from the Sawyer-Eliassen diagnoses calculated using the data from the $50^{\text {th }} \mathrm{h}$ to $90^{\text {th }} \mathrm{h}$.

Figure 3.7 Time evolution of azimuthal-mean tangential wind simulated by WRF from the $70^{\text {th }} \mathrm{h}$ to $84^{\text {th }} \mathrm{h}$.

Figure 3.8 Time evolution of horizontal wind speed at $1 \mathrm{~km}$ simulated by WRF from the $70^{\text {th }} \mathrm{h}$ to $84^{\text {th }} \mathrm{h}$. 
Figure 3.9 Tangential wind budget analyses averaged over the period from the $79^{\text {th }}$ $\mathrm{h}$ to $81^{\text {st }} \mathrm{h}$.

Figure 3.10 The radial profiles of tangential wind budget shown in Figure 3.8 at $500 \mathrm{~m}$ and $1000 \mathrm{~m}$, respectively.

Figure 3.11 Azimuthal-mean diabatic heating derived from the WRF simulations at different time from the $50^{\text {th }} \mathrm{h}$ to $80^{\text {th }} \mathrm{h}$.

Figure 3.12 Time-height variation of daibatic heating averaged over the radii of $60-80 \mathrm{~km}$.

Figure 3.13 Momentum forcing derived from the WRF simulation from $50^{\text {th }} \mathrm{h}$ to $80^{\text {th }} \mathrm{h}$.

Figure 3.14 (a) Total diabatic heating at the $79^{\text {th }} h$. (b): Eyewall diabatic heating with vertical velocity greater than $0.5 \mathrm{~m} / \mathrm{s}$. (c): Outer rainband diabatic heating with vertical velocity greater than $0.5 \mathrm{~m} / \mathrm{s}$.

Figure3.15 Tendencies of tangential wind at $500 \mathrm{~m}$ induced by eyewall and outer rainbands derived from the Sawyer-Eliassen diagnoses indicated by the blue and red colors, respectively. The tendencies induced by the diabatic heating are indicated by the dashed curves and the tendencies induced by the momentum forcings are indicated by the dotted curves. ..70

Figure 3.16 The same as Figure 3.15 but for the height of $1000 \mathrm{~m}$. .71

Figure 3.17 (a) Tendencies of tangential wind induced by the outer rainband heating with different strength (indicated by the different colors) averaged over the radii of 50-70 km subtracted by the corresponding tendency induced by the eyewall heating. (b): Ratio of the strongest outer rainband heating to the strongest eyewall heating for different experiments. .72

Figure 3.18 Other rainband diabatic heating (upper row), tendency of tangential wind induced by the rainband heating ( $\mathrm{mid}$ row), and divergence of radial flow induced by the rainband heating (bottom row) at different time.

Figure 3.19 Upper row: Outer rainband diabatic heating and the induced tendency of tangential wind and divergence of radial flow at the $78^{\text {th }} \mathrm{h}$. Bottom row: The same as the upper row but with the heating below $3 \mathrm{~km}$ being removed.

Figure 3.20 Time evolution of cloud water (a), rain water (b), relative humidity (c), equivalent potential temperature (d), difference of potential temperature 
between $3 \mathrm{~km}$ and $2 \mathrm{~km}(\mathrm{e})$, and surface buoyancy fluxes (f) averaged over the radii of $50-80 \mathrm{~km}$.

Figure 3.21 Subsidence (white contours) induced by the outer rainbad heating overlapped onto the vertical velocity (color shades) simulated by WRF. 76

Figure 3.22 Subsidence (white contours) induced by the eyewall heating overlapped with the vertical velocity (color shades) simulated by WRF.

Figure 3.23 Radial flow induced by the eyewall heating (black contours) and outer rainband heating (white contours) overlapped onto the vertical velocity simulated by WRF. Radial inflow and outflow are indicated by solid and dashed contours, respectively.

Figure 3.24 NPS of wavenumber 1, 2, 3, and sum of higher wavenumbers than 3 at the height of $2 \mathrm{~km}$ (shades) overlapped with the basic state PV radial gradient (contours). The blue and red contours indicate the PV radial gradient with value of $-5 \times 10^{-10}$ and $5 \times 10^{-10} \mathrm{~s}^{-1}$ respectively.

Figure 3.25 The same of Figure 3.24 but for NPS at $5 \mathrm{~km}$.

Figure 3.26 Radial-height plot of diabatic heating (shades) overlapped with convergence of radial flow (green contours) and symmetric contribution to the tendency of tangential wind at different time during ERC. The contours of radial flow convergence are: $-3 \times 10^{-4}$ and $-6 \times 10^{-4} \mathrm{~s}^{-1}$. The contours of tangential wind tendency are $1 \times 10^{-3}, 4 \times 10^{-3}$, and $7 \times 10^{-3} \mathrm{~ms}^{-2}$.

Figure 4.1 Vertical velocities at $5 \mathrm{~km}$ (left column) and tangential wind at $1 \mathrm{~km}$ (right column) from the control run, and YSU and MYNN experiments, respectively

Figure 4.2 Height-radius plots of tangential wind from the YSU experiment at different times from the $61^{\text {st }} \mathrm{h}$ to $93^{\text {rd }} \mathrm{h}$.

Figure 4.3 The same as Figure 4.2 but for the MYNN experiment.

Figure 4.4 Vertical velocity at the height of $5 \mathrm{~km}$ at the two instantaneous times during the first and second outer raniband activity from the MYNN and YSU experiments.

Figure 4.5 The same as Figure 4.4 but for horizontal wind speed at the height of 1 $\mathrm{km}$.

Figure 4.6 Tangential wind budget analyses (the same as Figure 3.9 but for the MYNN ex-periment) averaged over the period from the 70th $\mathrm{h}$ to 72nd h. 
Figure 4.7 Height-radius plot of azimuthal-mean diabatic heating (white contours), tangential wind (black contours), and net tendency of tangential wind at different times in the ERC period from the MYNN experiment. Note that the diabatic heating has been multiplied by a factor of 1000. The contour starts from 2 with an increment interval of 3

Figure 4.8 Tendency of tangential wind induced by the horizontal advection of absolute vorticity and vertical advection of tangential wind (color shades) and convergence of radial inflow (black contours with interval $-1,-0.5$, and $-0.11 / \mathrm{s}$ ) at the height of $1 \mathrm{~km}$ from the MYNN experiment. Note that both tangential wind tendency and convergence of radial inflow have been multiplied by a factor of 1000 .

Figure 4.9 Time evolution of (a) relative humidity at the height of $300 \mathrm{~m}$, (b) equivalent potential temperature at the height of $300 \mathrm{~m}$, (c) surface buoyancy fluxes, and (d) cloud water mixing ratio averaged over the radii of $60-100 \mathrm{~km}$.

Figure 4.10 Diabatic heating, surface latent heat fluxes, 10-m wind speed, and friction velocity at the $70^{\text {th }} \mathrm{h}$ and $85^{\text {th }} \mathrm{h}$ respectively from the MYNN experiment.

Figure 4.11 Diabatic heating (color shades) and vertical velocity (contours) at the $65^{\text {th }} \mathrm{h}$ and $85^{\text {th }} \mathrm{h}$ from the MYNN experiment where updraft and downdraft are indicated by black $(0.1$ and $0.5 \mathrm{~m} / \mathrm{s})$ and white contours $(-0.05 \mathrm{~m} / \mathrm{s})$, respectively.

Figure 4.12 Radial flow induced by the eyewall and outer rainband heating at the $65^{\text {th }} \mathrm{h}$ and $85^{\text {th }} \mathrm{h}$ from the MYNN experiment where the radial inflow and outflow induced by the eyewall heating are indicated by solid and dashed white contours and those induced by the outer rainband heating are indicated by solid and dashed black contours, respectively. The contour values of inflow and outflow are $-0.2,-1$, and 1,3 , and $5 \mathrm{~m} / \mathrm{s}$, respectively.

Figure 4.13 Diabatic heating, surface latent heat fluxes, 10-m wind speed, and friction velocity at the $75^{\text {th }} \mathrm{h}$ and $85^{\text {th }} \mathrm{h}$ respectively from the YSU experiment.

Figure 4.14 Radial distribution of diabatic heating and 2-m potential temperature during ERC events from the MYNN and YSU experiments.

Figure 4.15 Time evolution of vertical velocit at $5 \mathrm{~km}$ and tangnetial wind at $1 \mathrm{~km}$ from the control run and MYJ-BL2 experiment.

Figure 4.16 Height-radius plots of tangential wind from the MYJ-BL2 experiment at different times from the $61^{\text {st }} \mathrm{h}$ to $93^{\text {rd }} \mathrm{h}$. 
Figure 4.17 Radial distribution of diabatic heating, surface latent heat flux, 10-m wind speed, and 2-m potential temperature during ERC events from the MYJ-BL2 experiment.

Figure 4.18 Radial profiles of tangential wind (solid) and relative vorticity (dash) for control simulation (black) and sensitivity tests with double RMAX (green), stronger tangential winds outside RMAX ( $\mathrm{n}=1.35$; blue) and with an annulus vortex $(\mathrm{k}=1.05$; red $)$.

Figure 4.19 Time evolution of vertical velocity at $5 \mathrm{~km}$ and tangential wind at 1 $\mathrm{km}$ from the control run, annulus, doubling RMW, and large PV skirt experiments.

Figure 4.20 Height-radius plots of tangential wind at different times during the ERC period from the double RMW experiments.

Figure 4.21 Height-radius plots of tangential wind at different times during the ERC period from the large PV skirt experiments.

Figure 4.22 Time evolution of vertical velocity at $5 \mathrm{~km}$ and tangential wind at 1 $\mathrm{km}$ from the control run and large/small snow terminal velocity experiments.

Figure 4.23 Diabatic heating (color shades) and radial inflow (white contours, -3 $\mathrm{m} / \mathrm{s}$ and $-12 \mathrm{~m} / \mathrm{s}$ ) at the $78^{\text {th }} \mathrm{h}$ from the control run and large/small snow terminal velocity experiments.

Figure 4.24 Time evolution of vertical velocity at $5 \mathrm{~km}$ and tangential wind at 1 $\mathrm{km}$ from the control run and the no radiation experiment.

Figure 4.25 Time evolution of vertical velocity at $5 \mathrm{~km}$ and tangential wind at 1 $\mathrm{km}$ from the control run and sensitivity experiments with background wind of $5 \mathrm{~m} / \mathrm{s}$ and $10 \mathrm{~m} / \mathrm{s}$, respectively. The vertical black line in (a), (c), and (e) indicates the time for the height-radius structure analyses shown in Figure 4.26

Figure 4.26 Height-radius plots of diabatic heating at an arbitrary time (indicated in Figure 4.25) during the ERC period from the control run (a), experiments with background wind of $5 \mathrm{~m} / \mathrm{s}$ (b) and $10 \mathrm{~m} / \mathrm{s}$ (c).

Figure 4.27 Time evolution of vertical velocity at $5 \mathrm{~km}$ and tangential wind at 1 $\mathrm{km}$ from the three sensitivity experiments on the initial vortex with a large PV skirt, (a) without background wind, (b) with $5 \mathrm{~m} / \mathrm{s}$ prescribed background wind, and (c) with $10 \mathrm{~m} / \mathrm{s}$ prescribed background wind. ....135 


\section{ABBREVIATIONS AND ACRONYMS}

ERC

IB

MYJ

MYNN

NPS

PBL

PV

RMW

RMW

SEF

SGS

SL

SLPMIN

SST

TC

TKE

VRW

VTMAX

WISHE

WRF

YSU
Eyewall Replacement Cycle

Inertial-Buoyancy

Mellor-Yamada- Janjic Scheme

Mellor-Yamada-Nakanishi-Nino-2.5 level TKE scheme

Normalized Power Spectrum

Planetary Boundary Layer

Potential Vorticity

Radius of Maximum Mean Tangential Winds

Radius of Maximum Wind

Secondary Eyewall Formation

Sub-grid Scale

Surface Layer

Minimum-Sea-Level-Pressure

Sea Surface Temperature

Tropical Cyclone

Turbulent Kinetic Energy

Vortex Rossby Wave

Maximum Azimuthal Mean Tangential Winds

Wind-Induced Surface Heat Exchange

Weather Research and Forecasting Model

Yonsei University Scheme 


\section{Chapter 1 Introduction}

\subsection{Overview}

This dissertation focuses on a better understanding of physical mechanisms that control the eyewall replacement cycle (ERC) of tropical cyclones (TCs). The first section of this chapter describes the common features of ERC and presents the scientific questions of ERC that motivate this research. The second section gives a brief review of current understanding of ERC followed by a summary of the objectives and the structure of this dissertation.

\subsection{ERC of a TC}

Eyewall replacement cycle is a common phenomenon often associated with dramatic changes in TC intensity and inner-core structure. It was first documented and studied in detail from observations by Willoughby et al. (1982). The ERC begins with the formation of a partial or complete ring of convection with heavy precipitation outside the eyewall of a TC. This ring of convection usually possesses a well-defined local maximum of tangential winds and a convergence in low-level radial winds, very similar to the primary eyewall, and is thus generally called concentric or secondary eyewall. Figure 1.1 shows an example of the concentric eyewall in Hurricane Ivan (2004). In addition to the concentric eyewall, radar images often show that there is a precipitation free or weak precipitation annular zone that separates the outer ring of convection from the inner eyewall, often called moat (Houze et al. 2007). A moat is visible between the concentric eyewalls of Hurricane Ivan (2004) shown in figure 1.1. After the secondary eyewall 
forms, the inner eyewall stops developing and starts to weaken as the outer eyewall contracts and intensifies. The inner eyewall eventually collapses and the secondary eyewall assumes the role of its predecessor, which concludes an ERC.

While the formation of an outer eyewall may cause a TC to weaken temporarily, the wind maximum associated with the outer eyewall often broadens the hurricane wind field, and thereby enhances the integrated kinetic energy of a TC (Maclay et al. 2008). The increase of kinetic energy can have profound impacts on coastal regions if a TC is close to shore. A sudden expansion of TC force winds near landfall can affect a larger coastal area while reducing the preparation time. Even when a TC is a little farther from shore, the expended wind field is likely to lead to a greater storm surge (Irish et al. 2008). Moreover, the contraction of the outer eyewall near the end of an ERC can sometimes lead to a rapid intensification to result in a more intense TC than that prior to the ERC. This scenario was the case of Hurricane Andrew (1992), which intensified to a category-5 hurricane as it approached the southeast coast of Florida after an ERC (Willoughby and Black 1996; Landsea et al. 2004).

Satellite and radar observations show that intense TCs may undergo multiple ERCs during their lifetime. On the basis of a 10-yr data (1997-2006) of passive microwave satellite imagery, Hawkins and Helveston (2008) showed that more than $50 \%$ of all TCs that reach 120 kts have multiple eyewalls present during their lifespan. The percentage of TCs with concentric eyewalls varies by basins with the western Pacific the highest ( $\sim 80 \%)$, the southern Hemisphere the lowest $(\sim 30 \%)$, and the Atlantic $(\sim 40 \%)$ and the eastern Pacific $(\sim 60 \%)$ in between. 
Because of the dramatic intensity and structure changes associated with ERC and its frequent occurrence, the motivation to understand and forecast this phenomenon has remained high for decades. As will be reviewed in the next section, due to its intricate nature, the most basic question about ERC is still open: What are the leading physical mechanisms that control the formation of the secondary eyewall and the demise of the primary eyewall? Advancing our understanding of ERC and addressing various scientific issues associated with ERC are the goals and motivation of this dissertation research.

\subsection{Current understanding of ERC}

A complete theory of the ERC requires physical explanations of the formation of the secondary eyewall and the subsequent demise of the primary eyewall. A review of current understanding of these two ERC components is presented below.

\subsubsection{Secondary Eyewall Formation (SEF)}

Over the years, extensive research on SEF and ERC has yielded many hypotheses on SEF but some of them are now abandoned according to the recent studies, for example, the topographic forcing (Hawkins 1983), the asymmetric friction due to storm motion (Willoughby 1979), and the synoptic-scale forcings in the wind-induced surface heat exchange (WISHE) instability for triggering SEF (Nong and Emanuel 2003) are now approved to be the unnecessary conditions for SEF. The leading theories on SEF may be summarized as follows.

(a) Dynamical adjustment to latent heating outside the primary eyewall.

Using Eliassen's (1951) diagnostic technique, Shapiro and Willoughby (1982, hereafter SW82) calculated the secondary circulation induced by point sources of heat 
and momentum placed at different heights and different radial locations with respect to the radius of maximum wind (RMW) in balanced axisymmetric TC-like vortices. They showed that for a barolinic vortex the lower tropospheric heat sources close to the $0^{0} \mathrm{C}$ isotherm placed outside the RMW can generate the strongest positive tendency of tangential wind somewhere between the RMW and the heat source or at the source itself. If the pattern of tendencies were to persist for some time, then, a secondary maximum of tangential wind would form near the source radius and surrounding the original RMW. This mechanism may explain how an outer concentric eyewall can form and eventually replace the inner eyewall. In a recent study, Rozoff et al. (2012) evaluated the pioneering theoretical analysis of SW82 using the data generated from a full physics simulation of a real TC. They showed that the sustained azimuthal-mean latent heating outside the primary eyewall eventually leads to SEF facilitated by a broadening wind field, which enhances not only the frictional inflow but the inertial stability as well. Their result ultimately confirms that the SEF mechanism of SW82, which was obtained in a much simpler framework, is still relevant in a complicated realistic TC environment.

The importance of diabatic heating to SEF is also emphasized by a number of other studies. Using cloud resolving simulations of real TCs, Judt and Chen (2010) showed that the high potential vorticity (PV) generation and accumulation from the convective activities in the rainbands in the incipient outer eyewall region play a key role in SEF. Moon and Nolan (2010) demonstrated that the diabatic heating resulted from convective and stratiform precipitation in outer rainbands can induce a secondary wind maximum. They argued that the accelerated tangential wind can wrap around the entire vortex if the diabatic heating lasts long enough, which could lead to SEF. Wang (2009) also found that 
the enhanced latent heating in the outer rainbands favors the outward expansion of winds and SEF. Fang and Zhang (2012) further illustrated that on a beta-plane the shear effect can have a substantial impact on the asymmetric diabatic heating associated with the stratiform and convective precipitation that promotes SEF. Although all these studies showed convincing evidence to support SW82's pioneering theoretical analyses on SEF, they all eluded answering the question why SEF does not always occur considering the robust diabatic heating outside the RMW observed in almost all TCs.

(b) Asymmetric vortex interaction, vortex Rossby wave, and axisymmetrization.

Unlike SW82's mechanism of SEF, which involves a vertical secondary overturning circulation, Kuo et al. (2004 and 2008) showed that the concentric vorticity structure can be resulted from the interaction between a small/strong inner vortex (resembling a TC core) and asymmetric neighboring weak vortices (resembling the vorticities induced by convection outside RMW) through the axisymmetrizing process in a 2D nondivergent barotropic model. In the same 2D barotropic framework, Montgomery and Kallenback (1997) showed that the negative radial vorticity gradient outside RMW of the basic-state PV field supports the outward propagation of vortex Rossby waves (VRWs) until the waves slow down approaching the stagnation radius where the group velocity of VRWs goes to zero. Their calculation indicates that the maximum acceleration of tangential wind generated by wave-mean-flow interaction generally occurs outside the radius of initial asymmetry, suggesting that the wave-mean-flow interaction based on the VRW dynamics may provide a mechanism for SEF. This mechanism is confirmed by Martinez et al. (2010) who showed that in the same 2D framework the nonlinear 
evolution of asymmetric disturbances outside a strong vortex ring with a large vorticity skirt may relax to form concentric rings of enhanced vorticity that contain a secondary wind maximum. However, the vortex interaction and VRW mechanisms for SEF suffer serious limitations because of the 2D barotropic framework that excludes 3D dynamics, boundary layer friction, and convective processes. It is unclear how these mechanisms function in a real TC.

Terwey and Montgomery (2008) proposed a beta-skirt axisymmetrization mechanism that incorporates both barotropic dynamics and 3D convective processes. The beta-skirt owns its name since there is a weak but persistently nonzero radial gradient of azimuthal-mean vertical vorticity existing outside the primary eyewall of a TC and the spatial change of basic-state vorticity is often referred to 'beta' in geophysical fluid dynamics. Within the beta-skirt region, the activity of sporadic convection depends on convective available potential energy, convective inhibition, and filamentation time scale. With favorable conditions, which are determined by the beta length scale and filamentation time scale, the perturbation vorticity and kinetic energy of sporadic deep convection in the beta-skirt region can be transferred (or axisymmetrized) upscale into the azimuthal-mean flow through the anisotropic cascade process in a similar manner to eddy kinetic energy being directed upscale into the quasi-zonal jet in the classic 2D betaplane turbulence theory. Using data from a full physics 3D simulation, Terwey and Montgomery (2008) tested their hypothesis and confirmed that the beta-skirt axisymmetrization is responsible for the SEF in their simulation. However, questions remain. As shown by Mallen et al. (2005), all fully developed TCs typically have a betaskirt of vertical vorticity outside the main core, then, why does the beta-skirt 
axisymmetrization mechanism operate in some cases leading to SEF but fail to work in other cases?

(c) Unbalanced dynamics associated with the boundary layer processes.

The mechanisms for SEF reviewed in (a) and (b) are obtained in the balanced framework and essentially inviscid. In a recent study, Huang et al. (2012) showed that the SEF in their simulation of a real mature TC is intimately involved with the unbalanced boundary layer processes and undergoes a sequence of structural changes occurring in the outer-core region. It begins with a broadening of the tangential winds followed by an increase of boundary layer inflow underneath the zone of broadened tangential winds and the development of a convergence zone within and just above the boundary layer outside of the primary eyewall. This narrow region of convergence is where supergradient winds develop and initiates the eruption of moist air out of the boundary layer to foster deep convection that forms the root of secondary eyewall. Their result suggests that the unbalanced response in the boundary layer serves as an important mechanism for initiating and sustaining a ring of deep convection in a narrow supergradient wind zone outside the primary eyewall. Recent studies by Abarca and Montgomery (2013) and Kepert (2013) confirms Huang et al. (2012)'s hypothesis for SEF via a progressive boundary layer control of vortex dynamics in response to a broadening of the tangential wind field. Using an axisymmetric nonlinear slab boundary layer model, Abarca and Montgomery (2013) further showed that the boundary layer dynamics alone are capable of developing secondary wind maxima outside the primary eyewall. But Kepert (2013) concluded that the development of supergradient flow is not essential to the development 
of the outer maximum of surface convergence based on his study. Despite the robust signal of broadening of the tangential winds as a precursor to SEF, none of these studies addressed what initiates the broadening of the tangential winds, a key process in this mechanism. It is unclear if the broadening of the tangential winds is the cause for initiating a SEF or the outcome of an initiated SEF by other processes.

\subsubsection{Demise of Primary Eyewall}

Previous studies suggested that the demise of inner eyewall convection during an ERC is likely caused by a disruption of its transverse circulation during the contraction and intensification of the outer eyewall. Two distinct mechanisms for this disruption have been proposed.

(a) Cutting off the boundary layer inflow into the inner eyewall by the outer eyewall

By comparing observations of concentric eyewalls with theoretical findings of SW82, Willoughby et al. (1982) hypothesized that the outer eyewall creates hostile conditions for the inner eyewall by cutting off the boundary layer inflow into the inner eyewall. This hypothesis was investigated later by Zhou and Wang (2011b) who used the 3D full physics simulations of an idealized TC vortex undergoing an ERC. Their budget analyses of equivalent potential temperature generally support Willoughby et al. (1982)'s hypothesis about the demise of primary eyewall.

However, for a secondary eyewall with a large radius, the "cut-off" process appears to be inefficient, which cannot explain the observed pace of eyewall replacement.

(b) Subsidence over the inner eyewall induced by the outer eyewall 
Also on the basis of the findings of SW82, Willoughby et al. (1982;1990) suggested that as the outer eyewall intensifies and moves inward, the diabatic heating released from the deep convection of the outer eyewall could induce substantial subsidence over the inner eyewall region to inhibit the updrafts of the inner transverse circulation and eventually lead to the demise of the inner eyewall. However, the theoretical analyses done by Rozoff et al. (2008) do not support this hypothesis. By dividing a TC vortex into five radial regions, namely, the eye, inner eyewall, moat, outer eyewall, and far-field, Rozoff et al. (2008) were able to derive an analytical solution of the transverse circulation equation of a balanced vortex model. Their results show that the subsidence induced by the outer eyewall is primarily within the eye and the moat, and thus, does not directly weaken the inner eyewall updrafts. The conflict between Willoughby et al. (1982;1990)'s hypothesis and Rozoff et al. (2008)'s finding may be due to the fact that different diabatic forcings were prescribed in these studies. Moreover, both studies used the inviscid, axisymmetric, quasi-static balanced vortex model, which is highly idealized compared with a real TC, and therefore, it is worthwhile to further examine the mechanisms that control the demise of inner eyewall with more realistic diabatic forcings that can be readily obtained from $3 \mathrm{~d}$ full-physics TC simulations.

\subsection{Proposed Research}

As reviewed in the previous section, SEF and ERC are the outcome of a complicated internal interaction among TC vortex, diabatic heating associated with eyewall and rain-band convection, and boundary layer processes. However, the details of this interaction in governing the occurrence and course of SEF and ERC are still not well 
understood. Moreover, in numerical simulations, the realization of this interaction depends strongly on how the sub-grid scale (SGS) processes, in particular, cloud microphysics, turbulent mixing, and atmosphere-ocean interface energy and momentum exchange, are parameterized. To date, the impact of SGS model physics on SEF and ERC has not been addressed, but a thorough understanding of this important impact of SGS parameterizations is critical for a better understanding of SEF and ERC and an accurate prediction of this unique phenomenon and the associated change in storm intensity by numerical models.

The objectives of the present dissertation research are to advance our understanding of underlying mechanisms that control SEF and ERC and investigate the impact of SGS parameterizations on SEF and ERC in 3D full physics simulations. Specifically, this dissertation aims to (1) investigate the physical mechanisms underlying SEF and ERC, (2) tackle two crucial but yet unanswered questions of SEF: What is the role of outer rainband convection in the SEF? What physical processes lead to the formation and development of secondary maximum of tangential wind? and (3) address how SGS parameterizations affect SEF and ERC in 3D full physics numerical simulations. To achieve the research goals, a series of 3D full physics TC simulations has been conducted and will be presented in the dissertation. The structure of the dissertation is organized as follows. Chapter 2 describes the methodology including the configuration of the numerical model, model physics, the initial conditions, and methods used to process model outputs. Chapter 3 summarizes the evolution of the simulated TC in the control experiment and the spontaneously generated ERC in this run, followed by a detailed investigation of the dynamical processes leading to the SEF and ERC. In Chapter 4, a series of carefully de- 
signed sensitivity tests will be presented, which will be used to investigate the sensitivity of the simulated ERCs to model initialization, model resolution, and model physics including cloud microphysical, vertical turbulent mixing, and surface exchange parameterizations, and explore the mechanisms underlying the sensitivities. These experiments will also be used to further assessing our findings in Chapter 3. Conclusions, discussions and future work on ERCs will be summarized in Chapter 5. 


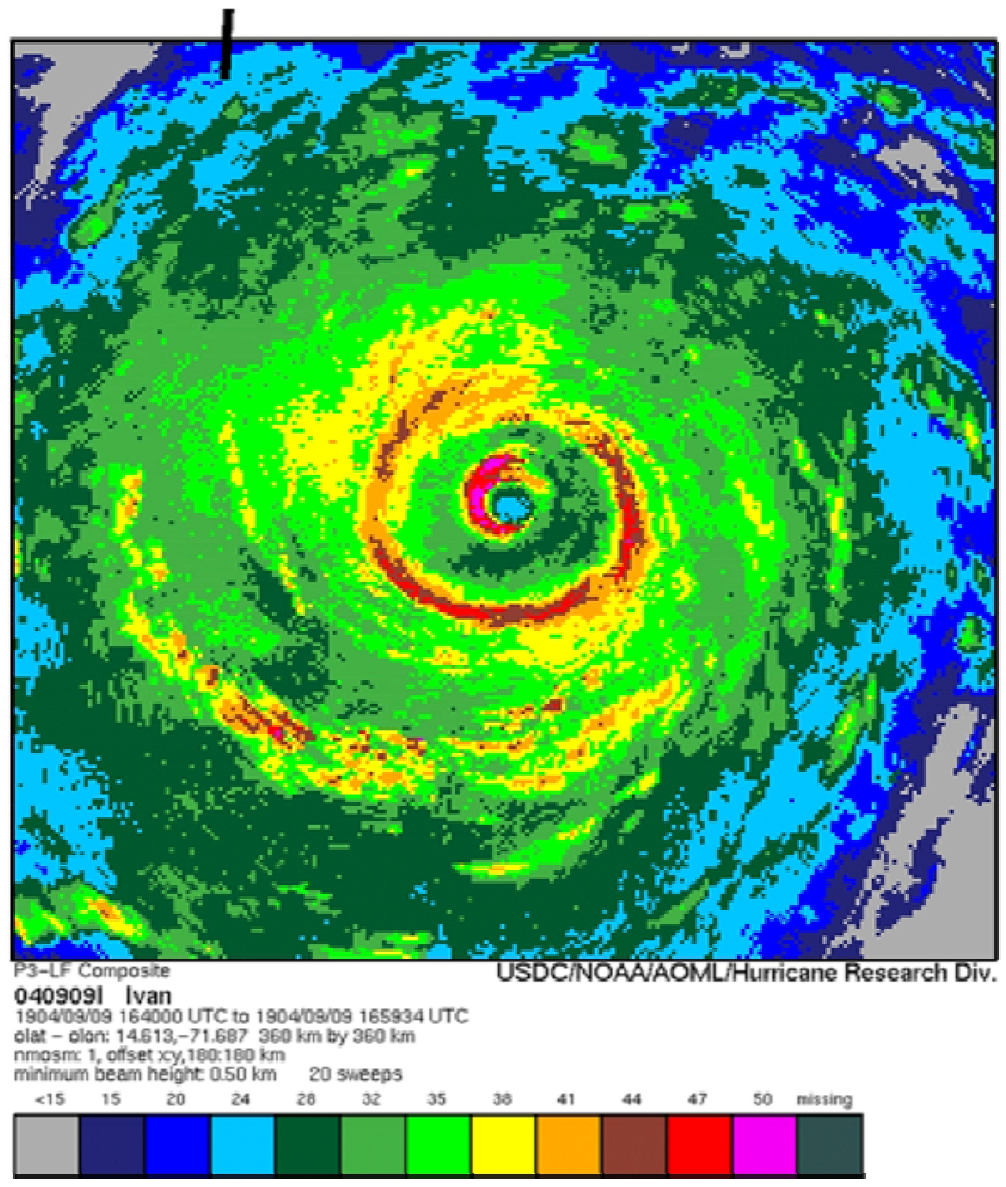

Figure 1.1 Research aircraft radar reflectivity composite of Ivan (2004) with concentric eyewalls (From Terwey and Montgomery, 2008). 


\section{Chapter 2 Methodology}

\subsection{Model configuration}

The Weather Research and Forecasting Model (WRF) with the Advanced Research WRF (ARW) dynamical core, version 3.3.1, is used for all simulations performed in this study. WRF is a fully compressible, nonhydrostatic model (Shamarock et al. 2008), and has been used successfully for both real and idealized TC simulations (Fierro et al. 2009; Nolan et al. 2009a,b; Stern and Nolan 2009; Wu et al. 2012; Zhu et al. 2013; Fovell et al. 2009; Hill and Lackmann 2009a,b). The WRF-ARW uses a terrain-following hydrostaticpressure (sigma) vertical coordinate (Laprise, 1992), and the primitive equations cast in the flux format are solved discretely on an Arakawa C-grid. In all simulations performed in this study, the model surface is set to be water only, and the sea surface temperature (SST) is uniformly fixed to $29{ }^{\circ} \mathrm{C}$. All map factors are set to 1 and the reciprocal of earth radius is set to 0 so that the computations are carried out on a Cartesian coordinate grid. All simulations are performed on an f-plane with a constant Coriolis parameter set to a value equivalent to that at $20^{\circ} \mathrm{N}$. The periodic lateral boundary condition and symmetric lateral boundary condition (free-slip wall) are applied to the western/eastern boundaries and southern/northern boundaries, respectively (Nolan 2011). All simulations contain three two-way nested square domains with a horizontal resolution of $18,6,2 \mathrm{~km}$, respectively. The outermost domain is configured sufficiently large (301x301 gridpoints) such that the lateral boundary conditions have a negligible impact on the dynamics of a simulated TC. The middle domain serves as a transition region and contains $181 \times 181$ gridpoints. The innermost nest contains $301 \times 301$ gridpoints giving a domain size of 
$600 \times 600 \mathrm{~km}$, which is large enough to cover the entire inner core (eyewall and main rainbands) of a simulated TC. The inner two domains are centered on the initial TC vortex and fixed throughout the simulation. The choice of motionless inner domains substantially improves the accuracy of tendency budget analyses frequently used in this study $^{1}$. All simulations use 47 vertical levels with a model top approximately at $26 \mathrm{~km}$ $(20 \mathrm{hPa})$. The 10 lowest half-levels ${ }^{2}$ are set at the heights approximately at $66 \mathrm{~m}, 200 \mathrm{~m}$, $335 \mathrm{~m}, 471 \mathrm{~m}, 610 \mathrm{~m}, 750 \mathrm{~m}, 893 \mathrm{~m}, 1037 \mathrm{~m}, 1209 \mathrm{~m}$, and $1459 \mathrm{~m}$ in the eyewall region. Grids with relatively high vertical resolutions are concentrated in the boundary layer (BL) and the upper troposphere (near $15-18 \mathrm{~km}$ ) since these two regions of the atmosphere have been found to be critical to the TC dynamics ${ }^{3}$. All simulations are run for 8 days, which allows the vortex structure to evolve sufficiently for the purposes of this study.

\subsection{Model physics}

All simulations use the Thompson microphysics scheme (Thompson et al. 2004). It includes 6 hydrometeor species: water vapor, cloud water, rain, ice, snow and graupel. In addition to the mixing ratios of each species, the scheme also predicts the number concentration of ice and rain. Observations show that the hydrometeors in the TC core are dominated by ice crystals and snow aggregates (Black and Hallett, 1986), and therefore,

\footnotetext{
${ }^{1}$ Since simulations are performed on a f-plane and there is no environmental flow, the simulated vortex does not drift far away from its initial position. Because of this, the innermost domain is able to cover the entire inner core region throughout the simulation even though the nest does not follow the vortex.

${ }^{2}$ Full levels are where the vertical velocity and geopotential are predicted, whereas half levels are where the horizontal velocities, temperature, moisture, and pressure are computed and stored.

${ }^{3}$ A TC is fueled by the air-sea surface flux exchanges of moist enthalpy via surface latent heat and sensible heat fluxes (e.g., Emanuel 1986), while the maintenance of the secondary circulation of a TC requires an uninterrupted anticyclonic ventilation flow in the upper troposphere (Zhang et al. 2002; Fierro et al. 2009), and hence, a better representation of these two regions can lead to a more realistic simulation of TCs.
} 
the ability to predict ice number concentration is desirable for a realistic TC simulation. Moreover, the treatment of snow growth in this scheme is designed to prevent or alleviate the problem of over-prediction of graupel, a problem that is shared by many singlemomentum microphysics schemes (Hong et al. 2004). These advantages make the Thompson scheme an appealing choice when limited computation resource is not sufficient for performing a set of numerical experiments with double-momentum or bin microphysics schemes. The Kain-Fritch cumulus scheme (Kain 2004) is used to represent subgrid-scale convection in the outermost domain, but it is inactivated in the two inner domains in which deep convective clouds are explicitly resolved. Horizontal diffusion is determined by the 2D Smagorinsky (1963) turbulence scheme. The Rapid Radiative Transfer Model (RRTM) radiation scheme (Mlawer et al. 1997) and the Dudhia scheme (Dudhia 1989) are used for longwave and shortwave radiative transfer calculation, respectively.

Fluxes and friction in the surface layer (SL) and vertical mixing in and above the PBL are parameterized using the Mellor-Yamada- Janjic (MYJ; Janjić 1996,2002;) suite of PBL and SL parameterizations (except for sensitivity tests of PBL schemes to be presented in chaper 4). The MYJ PBL scheme predicts the generation and redistribution of turbulent kinetic energy (TKE) and computes the eddy viscosities of momentum, heat and moisture from the predicted TKE to determine the SGS tendencies of these quantities. It is a "level 2.5" scheme in terms of the 4 "levels" of complexity for PBL schemes originally proposed by Mellor and Yamada (1974). The MYJ scheme is one of the PBL parameterization schemes that may be used for TC simulations recommended by Kepert 
(2012) because of its good performance in predicting near-surface wind profiles. The MYJ scheme available in WRF uses a wind speed-dependent formula (Charnok 1955) to determine the oceanic surface roughness $\left(\mathrm{z}_{0}\right)$. The strong dependence of the Charnock formula on wind speed results in a steady increase of the surface drag coefficient $\left(\mathrm{C}_{\mathrm{d}}\right)$ with the increase of wind speed. However, recent studies based on both hurricane dropsondes (Powell et al. 2003) and laboratory experiments (Donelan et al. 2004) show that $\mathrm{C}_{\mathrm{d}}$ does not increase unlimitedly with wind speeds, rather, it starts to level off at a certain large wind speed, for example, $30 \mathrm{~m} / \mathrm{s}$ according to Donelan et al. (2004), although there is a disagreement on the wind speed at which $C_{d}$ starts to level off. To account for this effect, the default Charnock formula of $z_{0}$ in the MYJ SL scheme is replaced by the one formulated by Davis et al. (2008) to match the findings by Donelan et al. (2004):

$$
\mathrm{z}_{0}=10 \exp \left(-\frac{10}{\mathrm{U}_{*}^{1 / 3}}\right),
$$

where $\mathrm{z}_{0}$ is limited within the range of $1.27 \times 10^{-7} \mathrm{~m} \ll \mathrm{z}_{0} \ll 2.85 \times 10^{-3} \mathrm{~m}$.

\subsection{Model initialization}

All simulations are initialized with an idealized vortex embedded in a quiescent background (except for sensitivity test with background winds to be presented in chapter 4) whose temperature and humidity profiles are specified by the non-SAL (Saharan air layer) sounding of Dunion and Marron (2008), which is an updated version of the original Jordan (1958) mean Caribbean hurricane-season sounding. The vortex and all the inner domains are initially placed at the center of the outermost domain. To shorten 
the evolution time of the initial vortex reaching its steady state, a category 1 vortex is used. The vortex has an axisymmetric structure with a maximum wind speed of 36.0 $m s^{-1}$ at surface at a radius of $45.0 \mathrm{~km}$. Its inner-core wind field realistically slopes outward with height. The pressure and temperature fields that hold the vortex wind field are in hydrostatic and gradient wind balance, which are derived following the iterative method proposed by Nolan and Montgomery (2002).

Following Wood and White (2011), the vortex tangential wind profile is determined parametrically by

$$
\mathrm{v}=\mathrm{v}_{\max } \frac{\rho^{\mathrm{k}}}{\left[1+\mathrm{kn}^{-1}\left(\rho^{\mathrm{n} / \lambda}-1\right)\right]^{\lambda}}, 0<k<n, \lambda>0
$$

where $\rho=\frac{r}{r_{\max }}, r_{\max }$ is the RMW; $v_{\max }$ is the maximum tangential wind; $\mathrm{K}, \mathrm{n}$, and $\lambda$ are the parameters that control the linearity or nonlinearity of the inner velocity profile between the vortex center and $r_{\max }$, the size of the decaying outer velocity profile, and the radial width of the velocity profile in the annular zone of the maximum, respectively. This parametric model is advantageous over the Rankine model since it produces a continuous tangential velocity profile and allows one to tune the wind profile with the three parameters (Wood and White 2011). In the control simulation, $\mathrm{k}=1, \mathrm{n}=1.6$ and $\lambda=0.2$ are chosen, which yields a solid body vortex profile similar to that of catergory 1 hurricane constructed by Nolan and Montgomery (2002). Figure 2.1 shows the radial profiles of tangential velocity and vorticity for this initial vortex. To prevent the vortex from having infinite radial extent, the profile determined by Equation 2.1 is multiplied by 
$\exp \left(-\left(\frac{r}{r_{\text {cut }}}\right)^{6}\right)$, which forces $v$ smoothly approaching to zero beyond a cutoff (in this case $600 \mathrm{~km}$ ) radius $\left(\mathrm{r}_{\text {cut }}\right)$. The adjusted profile is then extended into the vertical through an analytic function proposed by Nolan and Montgomery (2002),

$$
\mathrm{v}(\mathrm{z})=\mathrm{v} \times \exp \left[-\left(\frac{\mathrm{cz}}{\mathrm{z}_{\mathrm{top}}}\right)^{\alpha}\right] \times\left\{1-\gamma \exp \left[-\left(\frac{\mathrm{r}}{\delta+\mu \mathrm{z}}\right)^{\beta}\right]\right\}
$$

The first multiplicative factor on the right hand side (rhs) of Equation 2.2 causes the velocity field to decay appropriately with height consistent with what is observed in real TCs; $\mathrm{z}_{\mathrm{top}}$ is approximately the upper limit of the wind field. The second multiplicative factor has an $\mathrm{r}$ dependence that causes the inner-core wind field (i.e., the eyewall) to realistically slope outward with height. Parameter $\delta$ serves to prevent the last bracketed term from being undefined at $z=0$. Parameter $\gamma$ is chosen to be either one or zero depending on whether eyewall sloping is desired or not. In the Control simulation, the parameter settings are: $\mathrm{z}_{\mathrm{top}}=16 \mathrm{~km}, \alpha=2.0, \mathrm{c}=1.7, \beta=2.7, \delta=1$, and $\mu=4.0$.

2.4 Cylindrical coordinate used for analyses

Some of the data analyses used in this study, such as azimuthal average, Fourier decomposition of simulated fields, and budget analyses, require the data to be on the cylindrical coordinate centered at the vortex center. The cylindrical coordinate used in this study has an azimuthal resolution of 1 degree, a radial resolution of $2 \mathrm{~km}$, and a vertical resolution of $500 \mathrm{~m}$ within the radius and height of $300 \mathrm{~km}$ and $18 \mathrm{~km}$, respectively. All the data to be analyzed are interpolated from the model coordinate to the cylindrical coordinate. The (height-invariant) vortex center is defined as the geometric 
center, or centroid, of the surface pressure field (Braun et. al 2000). The location of the minimum pressure is used as the first guess of the center. A variational approach is then used to adjust the location of the center until the azimuthal variance of the pressure field at all radii between the center and the outer portion of the eyewall $(100 \mathrm{~km})$ is minimized. 


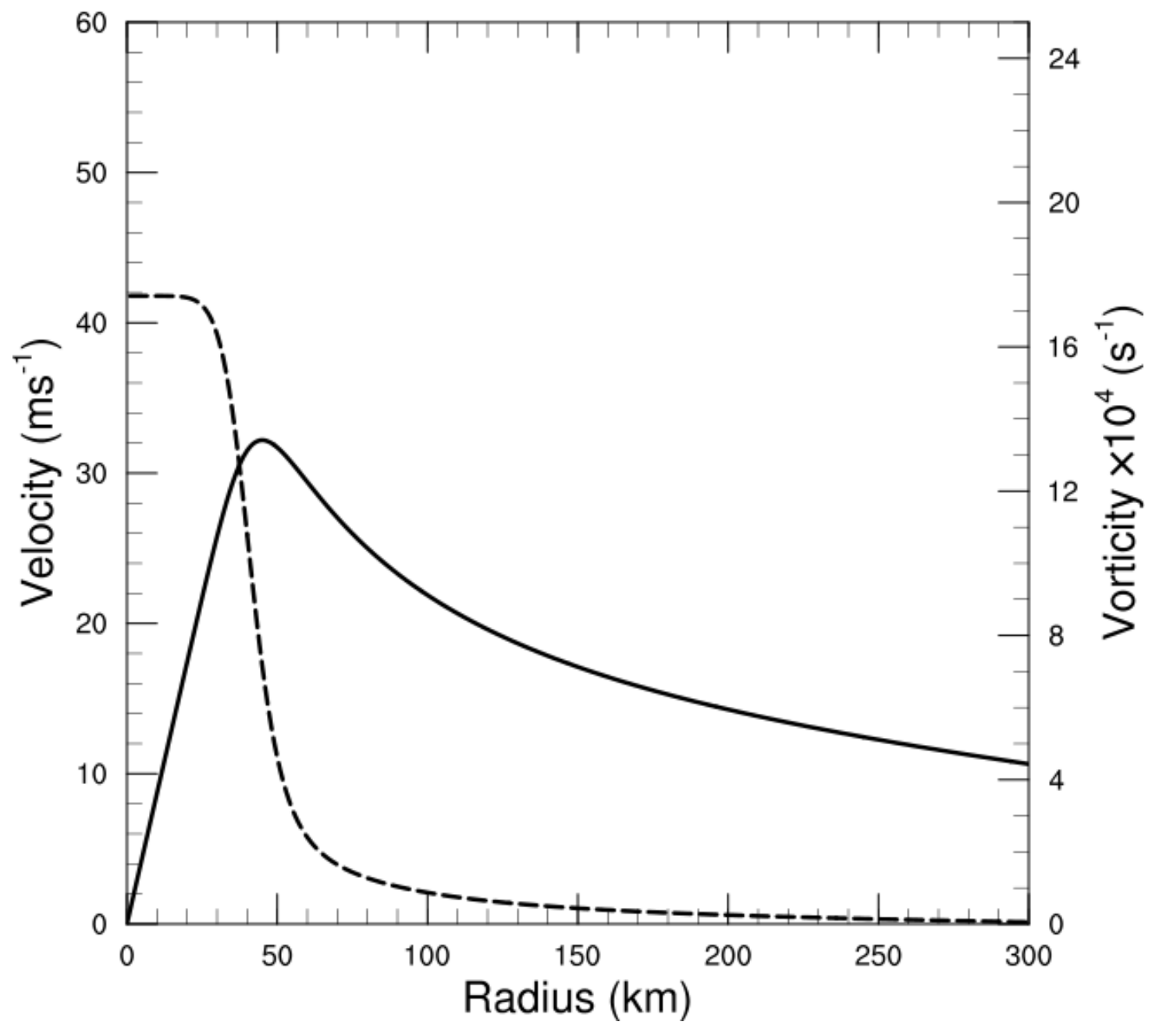

Figure 2.1 Radial profiles of tangential wind (solid) and relative vorticity (dash) for the initial vortex used in the control simulation. 


\section{Chapter 3 ERC in the control simulation}

3.1. Overview of the control simulation

Figure 3.1 shows the time series of minimum-sea-level-pressure (SLPMIN), maximum azimuthal mean tangential winds (VTMAX), and radius of maximum mean tangential winds (RMW) of the simulated vortex in the control simulation. These three metrics are widely used for evaluating the intensity and structure of a TC. Since the tangential winds of TCs typically maximize around the height of $1 \mathrm{~km}$ due to surface friction, VTMAX and RMW at $1 \mathrm{~km}$ level are presented in this figure. The initial SLPMIN is $990 \mathrm{hPa}$, and it remains nearly constant for the first 12 hours. Since no vertical motion nor any initial condensates are included in the initial vortex, during the first simulation hour the vortex is slowly spinning down (as seen in VTMAX) due to the surface friction. It takes about 6 hours till the developing organized deep convection can maintain itself. The RMW fluctuates about its initial value $(45 \mathrm{~km})$ by less than $7 \mathrm{~km}$ during this period. As the convection nearly saturates the core at mid-levels, which is required for storm intensification (Nolan et al., 2007), the SLPMIN begins to fall rapidly accompanied by a substantial increase of wind speed. This period approximately starts from $12^{\text {th }} \mathrm{h}$ and ends $66^{\text {th }} \mathrm{h}$ when the SLPMIN drops $900 \mathrm{hPa}$ and VTMAX reaches 96 $\mathrm{m} / \mathrm{s}$. The intensification rate in this period generally exceeds the criterion for Rapid Intensification (RI) $(15.4 \mathrm{~m} / \mathrm{s}$ in $24 \mathrm{~h}$ (Kaplan and DeMaria, 2003)) except for the period

between $36^{\text {th }} \mathrm{h}$ and $50^{\text {th }} \mathrm{h}$. The RMW rapidly decreases to about $22 \mathrm{~km}$ during the first 6 hours of this RI period, and then remains nearly constant until its end. Immediately following this RI period is a striking "V" shaped variation in the VTMAX, which 
terminates around $96^{\text {th }} \mathrm{h}$. The VTMAX decreases by $22 \mathrm{~m} / \mathrm{s}$ during the first half of this period and increases by $31 \mathrm{~m} / \mathrm{s}$ during the second half of this period. In the meantime, the RMW suddenly increases by $30 \mathrm{~km}$ at the midpoint of this $\mathrm{V}$-shaped period $(80 \mathrm{~h})$. Detailed examinations (to show later) show that during this period the simulated vortex undergoes an ERC, a reorganization process of TC inner core reviewed and summarized in Chapter 1. In many observational and simulation studies including this dissertation, events of dramatic change in VTMAX and sudden RMW increase are frequently used as preliminary evidences of TC's ERCs. The RMW rapidly decreases to $42 \mathrm{~km}$ during the next 8 hours after its sudden increase, indicating the contraction of the new dominant eyewall as the vortex intensifies. After that, RMW slightly increases as the vortex continues to intensify evidenced from the drop of SLPMIN till about $104^{\text {th }} \mathrm{h}$. During the next 34 hours following the intensification of the vertex after the ERC, approximately from $104^{\text {th }} \mathrm{h}$ to $138^{\text {th }} \mathrm{h}$, the vortex weakens by about $17 \mathrm{hPa}$ in SLPMIN and $15 \mathrm{~m} / \mathrm{s}$ in VTMAX. This weakening event is not completely clear but likely due to active mesovortices in the eyewall region (figures not shown), which may reduce storm intensity by asymmetric mixing (Kuo et al. 1999; Schubert et al. 1999). How eyewall mesovortices affect TC intensification is an interesting but complicated question. A thorough addressing this issue is beyond the scope of this dissertation study. After this weakening period, starting from $138^{\text {th }} \mathrm{h}$ the vortex reintensifies again and reaches its peak intensity (879 $\mathrm{hPa}$ in SLPMIN and $110 \mathrm{~m} / \mathrm{s}$ in VTMAX) around $176 \mathrm{~h}$. After that a quasisteady state appears to be reached and roughly maintained until the end of the simulation. 
Note that after the ERC event occurred around $72^{\text {nd }} h-86^{\text {th }} h$, the RMW generally takes on a slow increasing trend except for the period between $162^{\text {nd }} \mathrm{h}$ and $174^{\text {th }} \mathrm{h}$. The spatial expansion of the matured TC vortex appears to be in contradiction with the eyewall contraction predicted by axisymmetric balanced dynamics (SW82), however, the expansion of TC vortices is not a rare feature in 3D full physics numerical simulations (e.g., Hill and Lackman, 2009a; Terwey and Montgomery, 2008; Wang 2009). Wang (2009) suggested that the diabatic heating of outer rainbands may cause the expansion of a TC vortex. Detailed explanation of this phenomenon is beyond the scope of this study.

\subsection{Simulated ERC in the control experiment}

To clearly illustrate the detailed inner-core structure change associated with the ERC, Figure 3.2 shows a series of horizontal plots of synthetic radar reflectivity at the height of $1 \mathrm{~km}$ between $56^{\text {th }} \mathrm{h}$ to $86^{\text {th }} \mathrm{h}$ at an interval of 2 hours, which clearly depict how the outer rainbands develop and emerge into an intact outer ring of convection and how inner eyewall weakens and outer ring of convection develops to complete an ERC. Before the $60^{\text {th }} \mathrm{h}$, the spiral convective rain-bands are mainly active between the radii of $100 \mathrm{~km}$ and $160 \mathrm{~km}$. As time proceeds, the strong convective cells gradually approach closer to the TC center and start to appear in the region with radii between $60 \mathrm{~km}$ and 100 $\mathrm{km}$ around the $66^{\text {th }} \mathrm{h}$. After the $70^{\text {th }} \mathrm{h}$, the convective cells in this region rapidly develop in both their intensities and the areas that they cover. Meanwhile, the disconnected convective cells gradually consolidate into rainbands and eventually emerge into a ring of convection outside the primary eyewall with a radius of approximately $60 \mathrm{~km}$ at $78^{\text {th }} \mathrm{h}, 2$ hours right before the sudden increase of RMW (Figure 3.1). The $78^{\text {th }} \mathrm{h}$ is thus hereafter 
referred to as the "formation time" of the secondary eyewall, and the region with the radii between $60 \mathrm{~km}$ and $100 \mathrm{~km}$ hereafter is referred to as the "SEF region". At the $78^{\text {th }} \mathrm{h}$, a closed secondary concentric eyewall is clearly visible outside the inner eyewall. The formation and development of the secondary concentric eyewall is clearly reflected in the azimuthal-mean radial PV profile shown in Figure 3.3. At the $66^{\text {th }} \mathrm{h}$, a small local PV maximum is somewhat visible at the radius of $64 \mathrm{~km}$. It intensifies and contracts with time. By the time of $78^{\text {th }} \mathrm{h}$ when the closed secondary concentric eyewall forms, a clean secondary PV peak is shown in the PV profile that is separated from the primary eyewall PV maximum by the moat. The eyewall replacement follows the next 6 hours. It is noteworthy that the strong convective cells rarely reach inside the $55 \mathrm{~km}$ circle. This is due to the existence of a moat outside the inner eyewall before the SEF. The width of the moat at the height of $5 \mathrm{~km}$ is approximately $30 \mathrm{~km}$ in this case. With the development of the outer ring of convection, the inner eyewall begins to weaken around the $72^{\text {nd }} \mathrm{h}, 6$ hours before the SEF. This weakening appears to accelerate as the outer ring of convection contracts and intensifies. The inner eyewall eventually disappears around the $84^{\text {th }} \mathrm{h}$, approximately 4 hours after the sudden increase of RMW, and the ERC concludes when the newly developed eyewall takes the role of the primary eyewall of the vortex. The whole eyewall replacement process takes about 6 hours. The newly developed eyewall stops contracting at a radius about $20 \mathrm{~km}$ larger than the original RMW, leading to a larger new primary eyewall than the old one.

To gain further understanding of the basic kinematic and thermodynamic changes throughout the period preceding and accompanying the ERCs, the vertical velocity, 
tangential wind component, and radial wind component of the simulated vortex are further examined. Figure $3.4 \mathrm{a}$ shows the mid-level $(5 \mathrm{~km})$ azimuthal-mean vertical velocity. Weak subsidence starts to appear outside of the primary eyewall around the $52^{\text {nd }}$ $\mathrm{h}$ as the primary eyewall intensifies. This weak subsidence gradually develops into the so-called moat with a width approximately $10 \mathrm{~km}$ at this time. Starting from about the $60^{\text {th }} \mathrm{h}$, notable diffusive updrafts begin to develop between the radii of $100 \mathrm{~km}$ and 180 $\mathrm{km}$, indicating the development of asymmetric outer rain-bands. It appears that these outer rain-bands propagate inward with time but stay outside the moat region, and they gradually solidify into a more coherent structure around the $70^{\text {th }} \mathrm{h}$. After that, the inner rain-bands rapidly intensify and eventually evolve into the secondary eyewall. As the outer rainbands propagate inward, the moat also strengthens and slightly broadens but its width is confined by the primary eyewall and inner rain-bands. After the primary eyewall begins to lose its strength, the moat narrows and weakens substantially as the secondary concentric eyewall forms. It eventually merges into the subsidence in the eye as the inner eyewall disappears.

The radial structure of tangential wind also has a substantial change before and during the ERC. Figure $3.4 \mathrm{~b}$ shows the tangential wind at $1 \mathrm{~km}$ level. The tangential wind generally expands outward with time. The expansion of the tangential wind outside 150 $\mathrm{km}$ radius begins well before the $40^{\text {th }} \mathrm{h}$, likely due to the rapid intensification of the TC vortex. The expansion appears to gradually extend from the outer region to the inner region with time. The tangential wind in the SEF region begins to increase around the $60^{\text {th }} \mathrm{h}$, roughly the same time as the outer diffusive updrafts appear shown in Figure $3.4 \mathrm{a}$. 
The increase of tangential wind is accelerated as the updrafts emerge in this region after the $65^{\text {th }} \mathrm{h}$ (Figure3.4a), and is significantly enhanced after the updrafts begin to solidify around the $70^{\text {th }} \mathrm{h}$. A striking secondary tangential wind maximum first appears near the radius of $60 \mathrm{~km}$ around the $76^{\text {th }} \mathrm{h}, 2$ hours right before the first appearance of the intact secondary ring of convection (Figure3. 2). The vertical structure of the secondary maximum of tangential wind is shown in Figure 3.7 and will be discussed later.

Figure $3.4 \mathrm{c}$ shows the evolution of low-level $(500 \mathrm{~m})$ radial flow overlapped with its radial divergence. While the flow is generally inward outside of the primary eyewall, outward flow is seen in the eye throughout the simulation. Like the tangential wind shown in Figure 3.4b, low-level inflow generally expands outward with time except that it weakens within the moat region as the moat develops. As indicated by the divergence of radial flow (contours), there is a radial divergence in the moat, but a convergence outside the moat consistent with the weakening and intensification of low-level inflow within and beyond the moat region. It is worth noting that the increase of inflow in the SEF region appears to be more dramatic than that of tangential wind before the updrafts begin to solidify $(70 \mathrm{~h})$. The vertical structure of radial flow will be shown and discussed later in this section.

Although the numerical experiment presented above is from a 3D full physics simulation of an idealized TC-like vortex, the key features of the simulated ERC are consistent with those shown in observations. Sitkowski et al. (2011) performed excellent composite analyses of ERCs with flight-level data and satellite microwave imagery. The key features of the observed ERCs based on their study include: 1) the secondary maxima 
of tangential wind are evident before the concentric eyewalls have clearly emerged in the precipitation fields; 2) the storm intensity and the inner wind maximum begin to weaken as the inner-core structure organizes into concentric rings; 3) the reintensification of storms after the ERC can be quite dramatic and often brings storms to their maximum lifetime intensity; 4) the contraction of the outer wind maximum usually ceases at a radius larger than that of the inner wind maximum at the start of the ERC. In comparison with the observations, the simulated ERC presented above shows: 1) the secondary wind maximum occurs 2 hours before the concentric eyewalls clearly emerges; 2) the primary eyewall begins to weaken 6 hours before the SEF; 3) the reintensification of the vortex is quite dramatic although it does not bring the storm to reach its maximum lifetime intensity; 4) the outer eyewall stops contraction at a radius $\sim 20 \mathrm{~km}$ larger than the RMW of the primary eyewall. In short, the control simulation of an idealized TC-like vortex is able to capture the key features of the observed ERCs. This provides us the confidence that the simulated ERC can be used to address various unanswered issues associated with ERC.

\subsection{Axisymmetric balanced aspects of SEF and ERC}

SW82 first demonstrated that the evolution of a balanced symmetric TC-like vortex may be understood from the transverse circulation induced by point heat and momentum sources via Sawyer-Eliassen equation. Recent studies, such as Bui et al. (2009), Rozoff et al. (2012), and Sun et al. (2013) examined SW82's symmetric aspects of TCs by diagnosing the secondary circulation induced by the diabatic heating and frictional forcings using data from realistic 3D full physics simulations. These studies show that in many ways the 
transverse circulation induced by the symmetric latent heating and friction derived from 3D simulations during an ERC can match the azimuthal-mean secondary circulation directly simulated by $3 \mathrm{D}$ models. However, all these simulations used relatively low model resolutions. For example, the analyses by Bui et al. (2009) were based on the simulation that has a coarse horizontal resolution of $5 \mathrm{~km}$ and 24 levels in the vertical. Although the simulation analyzed by Rozoff et al. (2012) has a high horizontal resolution of $1 \mathrm{~km}$, it only has 35 levels in the vertical, in particular, the lowest model level in their simulation was set to the height of $600 \mathrm{~m}$, which can barely resolve the fine vertical structure of the lower part of the inflow layer of a TC that has been shown to be critical to SEF and ERC by many studies. In Sun et al. (2013)'s simulation, the vertical resolution increases to 40 levels, but they used a relatively low horizontal resolution of $3.6 \mathrm{~km}$. Although these studies provided some insight into SEF and ERC, the key processes that govern SEF and ERC have yet to be clearly identified. Therefore, there is a need to revisit the SW82 symmetric aspects of SEF and ERC using the realistic numerical data in which the boundary layer inflow of a TC is better resolved. Moreover, the diagnostic ability of the Sawyer-Elisassen equation has not been fully explored in these studies. In this study, we will show that the mechanisms of SEF and ERC can be well demonstrated from a balanced symmetric perspective using Sawyer-Elisassen diagnoses and tangential wind budget analyses. Finally, in all previous studies the Sawyer-Eliassen and tangential wind budget analyses were applied to a single simulation of a TC vortex. However, as it will be shown in the next chapter of this dissertation, the simulated SEF and ERC are sensitive to the changes in model physics and initial vortex. It is interesting to see how the diagnosed transverse circulation in the symmetric balanced framework responds to these changes 
and if the Sawyer-Eliassen and tangential wind budget analyses can shed any new light on the sensitivity of SEF and ERC to the changes in model physics and initial condition (the diagnoses of sensitivity experiments will be provided in the next chapter). In the next section, we will provide a detailed derivation of azimuthal-mean tangential wind budget equation and Sawyer-Eliassen equation and explain how to use 3D numerical data to perform the analyses.

\subsubsection{Tangential wind budget and Sawyer-Eliassen equation}

Under the assumption of small perturbation of air density, i.e., $\left|\rho^{\prime \prime} / \rho_{0}<<1\right|$, where $\rho_{0}$ is the horizontally averaged air density satisfying $\rho_{0}=\rho_{0}(z)$ and the superscript double-prime indicates perturbations away from the horizontal mean, the governing equations of the atmospheric flow in hydrostatic balance in the cylindrical coordinate may be written as

$$
\begin{aligned}
& \frac{\partial u}{\partial t}+u \frac{\partial u}{\partial r}+v \frac{\partial u}{r \partial \lambda}+w \frac{\partial u}{\partial z}-C=-\frac{1}{\rho_{0}} \frac{\partial p}{\partial r}+T S G S_{u} \\
& \frac{\partial v}{\partial t}+u \frac{\partial v}{\partial r}+v \frac{\partial v}{r \partial \lambda}+w \frac{\partial v}{\partial z}+u\left(f+\frac{v}{r}\right)=-\frac{1}{r \rho_{0}} \frac{\partial p}{\partial \lambda}+T S G S_{v}, \\
& g=-\frac{1}{\rho_{0}} \frac{\partial p}{\partial r}, \\
& \frac{\partial \theta}{\partial t}+u \frac{\partial \theta}{\partial r}+v \frac{\partial \theta}{r \partial \lambda}+w \frac{\partial \theta}{\partial z}=\dot{\theta}+T S G S_{\theta}, \\
& \frac{\partial\left(\rho_{0} r u\right)}{\partial r}+v \frac{\partial\left(\rho_{0} v\right)}{\partial \lambda}+\frac{\partial\left(\rho_{0} r w\right)}{\partial z}=0
\end{aligned}
$$


where $r, \lambda$, and $z$ represent the radial, azimuthal, and vertical coordinates; $u, v, w, \theta$, and $p$ are the model grid-box mean wind components in three directions, potential temperature, and atmospheric pressure, respectively; $g$ is the acceleration of gravity; $\dot{\theta}$ is the diabatic heating rate; $T S G S_{U}, T S G S_{V}$, and $T S G S_{\theta}$ denote the SGS tendencies for radial wind, tangential wind, and potential temperature, respectively. $C=v^{2} / r+f v$ is the sum of the centrifugal and Coriolis force. Equations (3.1)-(3.5) represent the radial and tangential wind budget equations, hydrostatic equation, thermodynamic equation, and continuity equation, respectively. By decomposing the atmospheric flow into the axisymmetric and asymmetric components, i.e., $S=\bar{S}+S^{\prime}$, where $S$ is a generic variable, overbar and prime indicate the azimuthal mean and the perturbation from the azimuthal mean, the governing equations for the mean axisymmetric flow can be written as,

$$
\begin{aligned}
& \frac{\partial \bar{u}}{\partial t}+\bar{u} \frac{\partial \bar{u}}{\partial r}+\bar{w} \frac{\partial \bar{u}}{\partial z}-\bar{C}=-\frac{1}{\rho_{0}} \frac{\partial \bar{p}}{\partial r}+\bar{F}_{r} \\
& \frac{\partial \bar{v}}{\partial t}+\bar{u} \bar{\zeta}_{a}+\bar{w} \frac{\partial \bar{v}}{\partial z}=\bar{F}_{\lambda} \\
& g=-\frac{1}{\rho_{0}} \frac{\partial \bar{p}}{\partial r} \\
& \frac{\partial \bar{\chi}}{\partial t}+\bar{u} \frac{\partial \bar{\chi}}{\partial r}+\bar{w} \frac{\partial \bar{\chi}}{\partial z}=-\bar{\chi}^{2} \bar{Q} \\
& \frac{\partial\left(\rho_{0} r \bar{u}\right)}{\partial r}+\frac{\partial\left(\rho_{0} r \bar{w}\right)}{\partial z}=0
\end{aligned}
$$


where $\theta$ has been replaced by $\theta=1 / \chi$ for convenience of derivation. $\varsigma_{a}=f+v / r+\partial v / \partial r$ is the vertical component of absolute vorticity. The forcing terms, $\bar{F}_{r}, \bar{F}_{\lambda}$, and $\bar{Q}$, resulted from both resolved and SGS processes can be represented as,

$$
\begin{aligned}
& \bar{F}_{r}=\overline{\overline{u^{\prime} \frac{\partial u^{\prime}}{\partial r}}}-\overline{v^{\prime} \frac{\partial u^{\prime}}{r \partial \lambda}}-\overline{w^{\prime} \frac{\partial u^{\prime}}{\partial z}}+\overline{\frac{v^{\prime 2}}{r}}+\overline{T S G S_{u}}, \\
& \bar{F}_{\lambda}=-\overline{u^{\prime} \varsigma_{a}^{\prime}}-\overline{v^{\prime} \frac{\partial v^{\prime}}{r \partial \lambda}}-\overline{w^{\prime} \frac{\partial v^{\prime}}{\partial z}}+\overline{T S G S_{v}}, \\
& \bar{Q}=\overline{\dot{\theta}}+\overline{u^{\prime} \frac{\partial \theta^{\prime}}{\partial r}}+\overline{v^{\prime} \frac{\partial \theta^{\prime}}{r \partial \lambda}}+\overline{w^{\prime} \frac{\partial \theta^{\prime}}{\partial z}}+\overline{T S G S_{\theta}}
\end{aligned}
$$

where the SGS tendencies and latent heating are determined by the activated model parameterizations in simulations and saved directly in the WRF output. $\overline{T S G S_{u}}, \overline{T S G S_{v}}$, and $\overline{T S G S_{\theta}}$ include both azimuthal-mean SGS vertical and horizontal diffusion. $\bar{\theta}$ is the sum of azimuthal-mean diabatic heating sources from microphysics and radiation physics. The azimuthal-mean tangential wind budget analyses performed in this study are based on Equation (3.7).

The Sawyer-Eliassen equation for the transverse circulation induced by the symmetric latent heating and friction can be obtained by assuming a strict gradient wind balance, which reduces the azimuthal-mean radial wind budget, Equation (3.6), to,

$$
\bar{C}=-\frac{1}{\rho_{0}} \frac{\partial p}{\partial r} .
$$


With a little algebra derivation, Smith et al. (2005) showed that Equation (3.14) can be rewritten in the format of,

$$
g \frac{\partial \bar{\chi}}{\partial r}+\frac{\partial(\bar{\chi} \bar{C})}{\partial z}=0
$$

The azimuthal-mean continuity equation, Equation (3.10), implies that the azimuthalmean radial wind and vertical velocity may be expressed in terms of a streamfunction $\psi$,

$$
\bar{u}=-\frac{1}{r \rho_{0}} \frac{\partial \bar{\psi}}{\partial z}, \quad \bar{w}=\frac{1}{r \rho_{0}} \frac{\partial \bar{\psi}}{\partial r} .
$$

By differentiating Equation (3.15) with respect to time, eliminating the time derivatives of $\bar{\chi}$ and $\bar{v}$ using Equations (3.7) and (3.9), and combining Equation (3.16), the SawyerEliassen equation can be obtained in the form of,

$$
\begin{aligned}
\frac{\partial}{\partial r}[ & \left.-g \frac{\partial \bar{\chi}}{\partial z} \frac{1}{r \rho_{o}} \frac{\partial \bar{\psi}}{\partial r}-\frac{\partial(\bar{\chi} \bar{C})}{\partial z} \frac{1}{r \rho_{o}} \frac{\partial \bar{\psi}}{\partial z}\right]+\frac{\partial}{\partial z}\left[\left(\bar{\xi} \bar{\chi}(\bar{\zeta}+f)+\bar{C} \frac{\partial \bar{\chi}}{\partial r}\right) \frac{1}{r \rho_{o}} \frac{\partial \bar{\psi}}{\partial z}-\frac{\partial(\bar{\chi} \bar{C})}{\partial z} \frac{1}{r \rho_{o}} \frac{\partial \bar{\psi}}{\partial r}\right] \\
& =g \frac{\partial}{\partial r}\left(\bar{\chi}^{2} \bar{Q}\right)+\frac{\partial}{\partial z}\left(\bar{C}^{2} \bar{\chi}^{2} \bar{Q}\right)-\frac{\partial}{\partial z}\left(\bar{\chi}_{\bar{\xi}} \bar{F}_{\lambda}\right),
\end{aligned}
$$

where $\xi=2 v / r+f$ is twice the local absolute angular velocity, and $\varsigma=v / r+\partial v / \partial r$ is the vertical component of relative vorticity.

To obtain the balanced response of a TC vortex to the prescribed axisymmetric diabatic heating and friction sources by solving the Sawyer-Eliassen equation, Equation (3.17), the azimuthal-mean tangential wind, potential temperature, air density, and forc- 
ing terms resulted from both resolved and SGS processes in a cylindrical coordinate are needed. All these quantities are directly available from WRF output except for air density, which is diagnosed from pressure, potential temperature, water vapor specific humidity, and hydrometeor content. The data in the model Cartesian coordinate saved at a 10-min interval are converted into the cylindrical coordinate defined in section 2.4 using vertical linear interpolation and horizontal bilinear interpolation.

The Sawyer-Eliassen equation is solved in a domain of $250 \mathrm{~km}$ (radius) $\times 18 \mathrm{~km}$ (height). To reduce the localized static instability that can be problematic in obtaining the solutions of balanced response, the azimuthal-mean tangential wind and potential temperature are smoothed slightly. All differentiation terms are calculated with the center finite difference, and the differential equation is solved with MUDPACK ${ }^{4}$. The boundary conditions for the Sawyer-Eliassen equation are set to $\psi=0$ at $r=0 \mathrm{~km}, \mathrm{z}=0 \mathrm{~km}$, and $\mathrm{z}$ $=18 \mathrm{~km}$; and $\frac{\partial \Psi}{\partial \mathrm{r}}=0$ at $\mathrm{r}=250 \mathrm{~km}$, same as those used in Fudeyasu and Wang (2010).

\subsubsection{Validation of Sawyer-Eliassen diagnoses}

The Sawyer-Eliassen equation was derived in the symmetric balanced framework. In SW82, it was applied to highly idealized TC-like vortices forced by point sources of heating and momentum. Thus, before applying the Sawyer-Eliassen analyses to realistically simulated TCs by a 3D full physics model, it is necessary to examine the extent to which the directly simulated vertical transverse circulation by WRF can be represented

\footnotetext{
${ }^{4}$ Multigrid Software For Elliptic Partial Differential Equations, which is a elliptic-partial-differentialequation solver using a multigrid method (Adams, 1999)
} 
by the diagnosed secondary circulation forced by the symmetric diabatic heating and friction obtained from the WRF simulation. To do so, careful comparisons between the simulated and diagnosed radial-height transverse circulations are made before, during, and after the SEF and ERC. As an example, Figure 3.5 shows the azimuthal-mean vertical velocities from the WRF simulation at the $65^{\text {th }} \mathrm{h}$ and $80^{\text {th }} \mathrm{h}$ respectively and those from the Sawyer-Eliassen diagnoses. There is a very good agreement between the WRF simulated azimuthal-mean vertical velocities and the Sawyer-Eliassen diagnoses in both magnitude and radial-vertical structure. In particular, the Sawyer-Eliassen diagnoses well capture the concentric eyewall structure and the moat between the inner and outer eyewalls at the $80^{\text {th }} \mathrm{h}$ simulated by WRF. To provide a further evaluation of how well the SawyerEliassen diagnoses can represent the simulated azimuthal-mean radial-vertical structure of vertical velocity, we computed the correlation coefficient between the simulated azimuthal-mean vertical velocities and those diagnosed from the Sawyer-Eliassen equation from $50^{\text {th }} \mathrm{h}$ to $90^{\text {th }} \mathrm{h}$, a period that covers the entire ERC from a time well before the SEF to a time when the vortex reintensifies after the completeness of the ERC. Figure3.6 shows the results and the associated confidence level of the statistical calculation. The directly simulated and diagnosed vertical velocities are well correlated except for the region near the model top. Below the height of $16 \mathrm{~km}$ and above the boundary layer, the correlation coefficients exceed 0.8 from the eyewall to the radii near the lateral boundaries, indicating a good representation of the vortex vertical velocity field by the SawyerEliassen diagnoses for most of the area involved in SEF and ERC. Even in the boundary layer, where the gradient wind balance is invalid due to the friction, the correlation coefficients are still greater than 0.6 , suggesting that to the first order approximation the Saw- 
yer-Eliassen analysis still applies. One may wonder why the Sawyer-Eliassen equation derived in a balanced symmetric framework can represent the transverse circulation in the boundary layer fairly well. This is because the boundary layer frictional effect has been indirectly included in the Sawyer-Eliassen equation through the forcing term $\bar{F}_{\lambda}$. Similar good correlations are also seen between the simulated radial flow and the SawyerEliassen diagnoses (not shown here). These statistical analyses give us the confidence that the Sawyer-Eliassen equation provides a useful analytical tool for investigating SEF and ERC in realistic 3D full physics simulations of a TC vortex despite the fact that it is derived in a symmetric balanced framework.

\subsubsection{Formation and development of secondary tangential wind maximum}

One of the key features of SEF and ERC is the formation and development of the secondary maximum of tangential wind associated with the outer concentric eyewall. Figure 3.7 shows the radial-vertical azimuthal-mean tangential wind field at different time from the $70^{\text {th }} \mathrm{h}$ to $84^{\text {th }} \mathrm{h}$, which clearly depicts the evolution of secondary wind maximum associated with the ERC. It begins with an expansion of tangential wind speed in the upper part of the boundary layer centered around $500-1000 \mathrm{~m}$ in the outer rainband region. With the expansion of the boundary layer tangential winds, the wind maximum of the primary eyewall starts to weaken and the secondary wind maximum gradually develops. The secondary tangential wind maximum reaches the magnitude comparable to that of the primary tangential wind maximum at about $80^{\text {th }} \mathrm{h}$. After that, the primary tangential wind maximum weakens rapidly and merges with the secondary tangential wind max-

imum. At about $82^{\text {nd }} h$, the primary tangential wind maximum completely disappears and 
the secondary tangential wind maximum assumes the role of its predecessor and continues to intensify. The evolution of horizontal instantaneous wind speed at $1 \mathrm{~km}$ is shown in Figure3.8. This figure indicates formation and development of a clean secondary maximum of the total wind speed associated with the ERC. What is depicted in Figs. 3.7 and 3.8 is consistent with recent studies by Huang et al. 2012, Abarca and Montgomery 2013, and Kepert 2013 who showed that the SEF is initiated with a broadening of the tangential winds followed by an increase of boundary layer inflow and the development of a convergence zone within and just above the boundary layer outside of the primary eyewall. However, although these studies correctly described the developing procedure that the tangential wind undergoes, these studies are unable to explain what causes the expansion of tangential winds outside the RMW at the top of the boundary layer. It should be noted that these studies attempted to seek mechanisms governing SEF and ERC from the perspective of unbalanced dynamics associated with the boundary layer processes. In this study we will show that the development of secondary maximum of tangential wind depicted in Figure 3.7 can be well addressed within the symmetric balanced framework. The detailed analyses will be provided below to demonstrate how the Sawyer-Eliassen diagnoses can be used to explain the expansion of tangential wind outside the RMW, the development of the secondary tangential wind maximum, and the demise of primary tangential wind maximum.

The evolution of tangential wind field shown in Figure 3.7 may be better understood by analyzing the tangential momentum budget equation, Equation 3.7. In the symmetric balanced framework, $\bar{F}_{\lambda}$ is treated as an external forcing. SW82 investigated 
how the tendencies of tangential wind (i.e., $\frac{\partial \bar{v}}{\partial t}=-\bar{u} \bar{\zeta}_{a}-\bar{w} \frac{\partial \bar{v}}{\partial z}$ ) change in response to the prescribed point sources of heating and momentum by solving the Sawyer-Eliassen equation. Since SW82 only focused on the tendency at the surface, $-\bar{u} \bar{\zeta}_{a}$ is the sole term that had been examined in their study considering that $\bar{w}(z=0)$ is zero. However, it will be shown shortly that both $-\bar{u} \bar{\zeta}_{a}$ and $-\bar{w} \frac{\partial \bar{v}}{\partial z}$ are the large terms in the boundary layer and generally tend to cancel each other due to the opposite signs. To understand the role of each term in the budget equation (Equation 3.7) in governing SEF and ERC, detailed budget analyses are performed using the WRF output, which provide many insights into the development of secondary wind maximum. The budget analyses averaged over $79^{\text {th }}-$ $81^{\text {st }} \mathrm{h}$ will be presented below as an example to illustrate the contribution of each term to the tendency of tangential wind at an important stage of the ERC.

The radial-vertical structure of each term in Equation 3.7 is shown in Figure 3.9, where the budget analyses are done every $10 \mathrm{~min}$, and then, averaged over $79^{\text {th }}-81^{\text {st }} \mathrm{h}$. The decomposition of the budget clearly reveals the role of each term in governing the evolution of tangential wind during the ERC. Here we want to emphasize a few key points that the budget analyses reveal. First, the positive and negative tendencies of tangential wind correspond well with the development of the secondary eyewall and the decay of the primary eyewall (Figure 3.9a). The net tendency ${ }^{5}$, however, is a small residual of a large cancellation between the two large tendencies induced by the resolved and SGS mixing processes (Figs. $3.9 \mathrm{~b}$ and 3.9c). It is, thus, not surprising that the simulated SEF

\footnotetext{
${ }^{5}$ Note that it has been multiplied by a factor of 10 for a clear presentation.
} 
and ERC show a strong sensitivity to model SGS turbulent mixing schemes. This may also explain why the SEF and ERCs in previous numerical studies show different characteristics. Part of the differences is likely caused by different model physics activated in the simulations. The large SGS contribution to the tendency of tangential wind poses a great challenge for a correct numerical forecasting of this phenomenon. Second, it is important to point out that the resolved processes are mainly responsible for generating positive tendencies in the boundary layer in the "SEF region" defined in Section 3.2, whereas the SGS tendencies in this region is negative, which is dominated by the SGS vertical diffusion (Figure 3.9h). The tendency induced by SGS horizontal diffusion is nearly 20 times smaller than that of SGS vertical diffusion (Figure 3.9i). Since the development of a secondary maximum of tangential wind requires a positive tendency, this result suggests that the resolved processes are the driving force for SEF and ERC and the SGS tendency may be considered as a response to such changes in resolved processes. Therefore, the resolved processes are the keys to understand SEF and ERC. For this reason, in the remaining of this chapter, the focus of analyses will be on the resolved processes. However, since the SGS tendency has a magnitude comparable to that of resolved tendency, the SGS parameterizations can have a profound impact on the occurrence and course of SEF and ERC, which will be discussed in detail in the next chapter. Third, the resolved horizontal advection term $\left(-\bar{u} \bar{\zeta}_{a}\right.$, Figure 3.9d) and vertical advection term $\left(-\bar{w} \frac{\partial \bar{v}}{\partial z}\right.$, Figure3.9e) are the two leading contributions in the budget but have opposite signs within and above the boundary layer. The cancellation of the two terms (Figure 3.9f), however, results in the positive tendencies in the boundary layer in the "SEF region". The resolved 
asymmetric eddy contribution (Figure 3.9g, multiplied by a factor of 4 for a clear presentation) is much smaller than the net symmetric contribution $\left(-\bar{u} \bar{\zeta}_{a}-\bar{w} \frac{\partial \bar{v}}{\partial z}\right)$, indicating that it may only have a minor effect on SEF and ERC. It should also be pointed out that the resolved asymmetric eddy term is generally negative in the boundary layer with two centers near the eyewall and the radius of $50 \mathrm{~km}$, which are likely caused by the eyewall and rainband perturbations. The negative tendency in the boundary layer induced by the asymmetric eddies suggests that it is not a driving force for the development of the secondary maximum of tangential wind associated with SEF and ERC.

To better illustrate the budget of azimuthal-mean tangential wind, the radial profiles of each term shown in Figure 3.9 at $500 \mathrm{~m}$ and $1000 \mathrm{~m}$ are plotted in Figure3.10. The tendency directly calculated from the WRF simulated tangential wind (black curve in Figs. 3.10a and 3.10c) does not exactly match the sum of all terms associated with the resolved and SGS processes (red curve in Figs. 3.10a and 3.10c), which is understandable due to the 10-min interval of budget calculation. But the fact that the two tendencies have similar radial structure and magnitude suggests that the 10-min budget analyses are fairly accurate, giving us extra confidence that the results gained from the analyses are reliable. The tendencies induced by the resolved processes in the "SEF region" at $500 \mathrm{~m}$ and 1000 $\mathrm{m}$ are both positive (blue curve in Figs. 3.10a and 3.10c), but for different reasons due to the sign change of the two leading terms $\left(-\bar{u} \bar{\zeta}_{a}\right.$ and $\left.-\bar{w} \frac{\partial \bar{v}}{\partial z}\right)$ in the budget. At $500 \mathrm{~m}$, terms $-\bar{u} \bar{\zeta}_{a}$ and $-\bar{w} \frac{\partial \bar{v}}{\partial z}$ are consistently positive and negative respectively in the "SEF 
region" with peak at the radius of $\sim 50 \mathrm{~km}$; whereas at $1000 \mathrm{~m}-\bar{u} \bar{\varsigma}_{a}$ and $-\bar{w} \frac{\partial \bar{v}}{\partial z}$ change their sign at the radius of $\sim 50 \mathrm{~km}$. The decomposition of tangential wind budget shown in Figs. 3.9 and 3.10 indicates that the intuition of SW82 in analyzing the term, $-\bar{u} \bar{\zeta}_{a}$, is scientifically right although it was not possible for them to perform a detailed budget analysis at that time. It is one of the leading terms in the budget and can induce positive tendency in the "SEF region". However, as we showed here, aside from the highly idealized vortex and forcing used by SW82, their analyses are incomplete in that they did not consider another important term, $-\bar{w} \frac{\partial \bar{v}}{\partial z}$, which tends to cancel the effect of $-\bar{u} \bar{\varsigma}_{a}$. In addition, only considering the tangential wind tendency at the surface is insufficient since both $-\bar{u} \bar{\zeta}_{a}$ and $-\bar{w} \frac{\partial \bar{v}}{\partial z}$ change their sign in the boundary layer as shown in Figs. 3.9 and 3.10. For this reason, in this study we shall treat the term $-\bar{u} \bar{\varsigma}_{a}-\bar{w} \frac{\partial \bar{v}}{\partial z}$ as a whole (due to the large cancellation between them) to understand the processes that control SEF and ERC.

The Sawyer-Eliassen equation is a powerful diagnostic tool. It allows us to look into the response of transverse circulation and the induced tangential wind tendency to different diabatic heating and momentum forcings in detail. To better understand the forced transverse circulation and the associated tangential wind tendency, it is useful to first examine how the heating and momentum forcings evolve during the SEF and ERC. Figure 3.11 shows the azimuthal-mean diabatic heating derived from the WRF simulations at different time from $50^{\text {th }} \mathrm{h}$ to $80^{\text {th }} \mathrm{h}$. The diabatic heating is mainly concentrated in the 
eyewall as expected, but it is also seen outside the eyewall mainly associated with the convective activities in the outer rainbands. The outer rainband heating is separated from the eyewall heating by the moat. As indicated by the figures, for a long period of time before the SEF, the outer rainband heating stays weak till the $72^{\text {nd }} h$. After that, there is a rapid enhancement of outer rainband heating near the radii of $\sim 50-70 \mathrm{~km}$, which eventually develops into the secondary eyewall. To clearly illustrate the development of outer rainband heating, Figure 3.12 shows the time-height variation of diabatic heating averaged over the radii of $60-80 \mathrm{~km}$ where most of outer rainband heating is located according to Figure 3.11. It clearly shows that there is a sudden increase in diabatic heating around the $72^{\text {nd }} \mathrm{h}$. It starts with an enhancement of low convection just above the boundary layer and quickly extends to the mid- and upper-troposphere. We will show shortly that this sudden increase in outer rainband heating is the key process that governs the $\mathrm{SEF}$ and ERC. The decrease of outer rainband heating after $82^{\text {nd }} \mathrm{h}$ shown in Figure3.12 is due to the fact that the convection moves radially inward and eventually becomes the outer eyewall at the radius of $\sim 50 \mathrm{~km}$. The momentum forcing derived from the WRF simulation at different time is shown in Figure 3.13. As expected the momentum forcing is mainly concentrated in the boundary layer in vicinity of the eyewall before the SEF. It extends radially outward as the convection associated with the outer rainbands develops.

To seek and identify the key processes that govern SEF and ERC, several partitions of the total heating and momentum forcings are carried out to examine how diabatic heating and momentum forcings lead to the formation and development of secondary tangential wind maximum. These include the decomposition of forcings in terms of microphysi- 
cal processes, different radial ranges, and eyewall/rainbands. It turns out that the interaction between eyewall and outer rainband heating plays a key role in governing the evolution of secondary tangential wind maximum. To clearly demonstrate the underlying mechanism and remove unnecessary complication, for this analysis only heating associated with vertical velocity greater than $0.5 \mathrm{~m} / \mathrm{s}$ is considered. This allows us to cleanly separate the eyewall heating and outer rainband heating as illustrated by Figure 3.14. The same method is used to separate the eyewall and outer rainband momentum forcing. The separated forcings are then used to solve the Sawyer-Eliassen equation so that the contribution from eyewall and outer rainband heating and momentum forcing to the forced transverse circulation and tangential wind tendency can be quantified.

Figs. 3.15 and 3.16 show the symmetric contribution $\left(-\bar{u} \bar{\zeta}_{a}-\bar{w} \frac{\partial \bar{v}}{\partial z}\right)$ to the tendency of tangential wind at $500 \mathrm{~m}$ and $1000 \mathrm{~m}$ induced by the eyewall and outer rainband diabatic heating and momentum forcing derived from the Sawyer-Eliassen diagnoses. Although the magnitudes are different, both figures share similar characteristics. The tendency generated by the eyewall forcing is negative in the eye but it quickly changes to positive with a large peak at the inner edge of the eyewall. The positive tendency drops quickly and then gradually slopes radially outward and eventually approaches zero at a sufficiently large radius. These features share the similar characteristics to the surface tendency induced by the point heating source placed at the vicinity of the eyewall found by SW82. It is apparent that the eyewall forcing alone will never induce a secondary tangential wind maximum. The tendency generated by the outer rainband forcing remains small till the $72^{\text {nd }} \mathrm{h}$, but after that, its magnitude increases rapidly over the radial range of 
$50-80 \mathrm{~km}$ where the outer rainband activity is the strongest. The negative value is at the inward edge of the tendency induced by the outer rainband forcing but is mainly confined in the lower boundary layer. At the height of $1000 \mathrm{~m}$, it becomes very small. This negative tendency tends to cancel the positive tendency induced by the eyewall forcing, which may serve to separate the secondary tangential wind maximum from the primary wind maximum. It is clear that the large positive tendency induced by the outer rainband forcing is the leading cause for the secondary tangential wind maximum. This is more apparent at the height of $1000 \mathrm{~m}$, which may explain why the tangential wind peaks at the height of $1000 \mathrm{~m}$ shown in Figure 3.7. Note that the large positive tendency in the inner eyewall induced by the eyewall forcing reduces dramatically at the height of $1000 \mathrm{~m}$, which is consistent with the decrease of primary tangential wind during the ERC. Figs. 3.15 and 3.16 also indicate that the tendency induced by the momentum forcing is much smaller than the diabatic heating induced tendency, suggesting that the momentum forcing mainly associated with friction is of secondary importance to SEF and ERC at least from the symmetric perspective.

The above Sawyer-Eliassen diagnoses on the symmetric contribution to the tendency of tangential wind reveal an important role of outer rainband heating in governing SEF and ERC. Comparison of Figs. 3.15, 3.16, 3.11, and 3.12 suggests that although outer rainband activity always exists during the evolution of a $\mathrm{TC}$, it must reach a certain strength before it can initiate and drive the secondary tangential wind maximum via horizontal advection of absolute vorticity and vertical advection of tangential wind (i.e., $\left.-\bar{u} \bar{\zeta}_{a}-\bar{w} \frac{\partial \bar{v}}{\partial z}\right)$. To better illustrate this mechanism, a series of Sawyer-Eliassen experi- 
ments are conducted using the artificially reduced outer rainband heating. Figure 3.17 shows the time series of the tendencies of tangential wind at $1000 \mathrm{~m}$ induced by the outer rainband heating with different strength averaged over the radii of 50-80 $\mathrm{km}$ subtracted by the tendency induced by the eyewall heating, a quantity that may be used to assess the development of secondary tangential wind maximum; and the time series of the ratio of the strongest outer rainband heating over the radius range between $50 \mathrm{~km}$ and $80 \mathrm{~km}$ at a certain time to the strongest eyewall heating, a quantity used here to evaluate the strength of outer rainband heating relative to that of eyewall heating. In the control simulation, the strongest rainband heating remains negligible compared with that of eyewall heating till the $69^{\text {th }} \mathrm{h}$ when the ratio of the strongest outer rainband heating to that of eyewall heating suddenly jumps to about $20 \%$. After that the ratio stays high and gradually increases with the strengthening of outer rainband heating and weakening of eyewall heating. The difference of the tendencies induced by the outer rainband heating and eyewall heating starts to increase about two hours later. This time delay is likely due to the fact the tendency is the integrated result of all heating forcing and suggests that the outer rainband heating is the cause of the formation of the secondary tangential wind maximum. The result also indicates that the strength of outer rainband heating must reach a certain level relative to the eyewall heating to cause SEF and that the ratio of the strongest outer rainband heating to that of eyewall heating may provide a good and practical predictor for a timely forecast of SEF and ERC. The Sawyer-Eliassen experiments suggest that SEF and ERC may still occur when the strength of outer rainband heating reduces to $10 \%$ of that of eyewall heating. Note that in this estimation the eyewall heating remains the same as that in the control simulation. We will show evidence and discuss shortly that the outer rainband heat- 
ing substantially weakens the eyewall as the secondary eyewall develops. This feedback is not considered in the Sawyer-Eliassen experiments. Thus, the strength of eyewall heating is underestimated in the reduced outer rainband heating experiments. Based on this consideration, it may not be possible to initiate SEF and ERC if the strength of outer rainband heating is less than $10 \%$ of that of eyewall heating. We have carefully examined the other periods of the control simulation and all the sensitivity experiments (to be shown in the next chapter). It is fairly consistent that the ratio of the strongest outer rainband heating to that of eyewall heating remains below $10 \%$ when there is no SEF and ERC, whereas the ratio is consistently above $10 \%$ for all ERC events observed in our simulations.

\subsubsection{Development of outer rainband convection during ERC}

Previous analyses demonstrate the crucial role of outer rainband convection in SEF and ERC. One question has yet to be addressed is the sudden enhancement of outer rainband convection, which has been shown to be necessary for initiating and driving SEF and ERC. The answer to this question may also clarify why in some other times outer rainband convection fails to promote SEF and ERC. Again we attempt to investigate this issue in the same symmetric balanced framework. To seek the mechanism underlying the development of outer rainband convection and its relation to SEF, we carefully examined the diabatic heating and the forced transverse circulation diagnosed by the sawyerEliassen equation before, during, and after SEF. To summarize our findings, Figure 3.18 shows the diabatic heating, the induced symmetric contribution to the tangential wind tendency, and the induced divergence of radial flow at different time during SEF. At the 
$69^{\text {th }} \mathrm{h}$ just before SEF, the weak outer rainband convection is seen in the mid-troposphere. As expected, the convection induces convergence and divergence of radial flow at the base and upper part of the convection (Figure 3.18c1), respectively. The convection also accelerates tangential wind near the base of the convection via horizontal advection of absolute vorticity and vertical advection of tangential wind $\left(-\bar{u} \bar{\zeta}_{a}-\bar{w} \frac{\partial \bar{v}}{\partial z}\right)$. At the $71^{\text {st }} \mathrm{h}$, convection extends down to the low troposphere. It is interesting to see that the convergence of radial inflow and acceleration of tangential wind also extend down to the base of the convection. Three hours later, the weak sporadic convection develops into solid convection extending from the top of the boundary layer upward to the tropopause. As a response, the divergence of radial flow and tangential wind tendency show an interesting vertical structure: two strong elongated zones of convergence and divergence of radial flow are seen at the radially outward and inward edge of the convection with the convergence extending down into the boundary layer (Figure 3.18c3); there is a jet of large positive tendency of tangential wind in the upper part of the boundary layer and it gets stronger as the rainbad convection intensifies (Figs. 3.18b3 and 3.18b4). As shown previously, this jet of positive tendency of tangential wind is responsible for the formation and development of secondary maximum of tangential wind. To show the importance of low level convection to the generation of large positive tendency of tangential wind and strong convergence of radial inflow in the boundary layer, we examined the transverse circulation forced by the same outer rainband heating but with the heating below $3 \mathrm{~km}$ being removed. As an example, Figure 3.19 compares the diagnosed symmetric contribution to the tangential wind tendency and divergence of radial flow forced by the outer 
rainband heating with and without heating below $3 \mathrm{~km}$. It clearly shows that without low level convection the large positive tendency of tangential wind and strong convergence of radial inflow in the boundary layer nearly disappear, indicating the crucial role of low level convection in SEF.

What shown in Figs. 3.18 and 3.19 suggests a positive feedback among low-level convection, convergence of radial inflow, and acceleration of tangential wind in the upper boundary layer that leads to the development of outer rainband convection and SEF, which may be described as follows. The sporadic convection in the outer rainband region induces the convergence of radial inflow and accelerates the tangential wind at the base of (or just beneath) convection, which can cause the increase of moisture in the low troposphere. The increased moist instability favors the development of low-level convection, which, according to the Sawyer-SEliassen diagnoses, can result in convergence of radial inflow and acceleration of tangential wind in the boundary layer. While the former leads to the effective transport of moisture into the convection, the latter can enhance surface evaporation. Both processes can foster further development of convection. This can cause runaway interaction leading to the development of outer rainband convection and formation of secondary maximum of tangential wind. To provide further evidence to support this positive feedback mechanism of SEF, Figure 3.20 shows the time evolution of cloud water, rain water, relative humidity, equivalent potential temperature, low troposphere stability, and surface buoyancy flux averaged over the radii of 50-80 km. As indicated by cloud water (Figure 3.20a), there is mid-tropospheric convection $(4-5 \mathrm{~km})$ existing long before SEF. This rainband convection is always seen during the evolution of a TC but 
may not directly link to SEF. At about $60^{\text {th }} \mathrm{h}$, weak shallow convection starts to develop possibly due to the convergence of radial inflow induced by the mid-troposphere convection as discussed previously and could also be partially attributed to the moistening of lower troposphere by the precipitation of mid-tropospheric convection. As indicated by Figs. $3.20 \mathrm{~b}$ and $3.20 \mathrm{c}$, there is light rainfall before $60^{\text {th }} \mathrm{h}$, which appears to be related to the positive perturbation of relative humidity in low-mid troposphere. The increase of low tropospheric moisture favors the development of shallow convection. Once sufficient shallow convection is initiated, its induced convergence of radial inflow and acceleration of tangential wind in the boundary layer can enhance surface evaporation (Figure 3.20f) to result in the increase of relative humidity or equivalent potential temperature in the low troposphere, which favors the further development of convection. Note that in this particular case, the lower tropospheric stability measured by the difference of potential temperature between $3 \mathrm{~km}$ and $2 \mathrm{~km}$ in the outer rainband region does not change much, indicating that the enhanced moist instability leading to SEF is mainly due to the increased low level moisture through the proposed positive feedback among low level convection, convergence of radial inflow and acceleration of tangential wind in the boundary layer, and surface evaporation.

\subsubsection{Interaction between eyewall and outer rainbands during ERC}

Observations and many previous numerical simulations show that the inner eyewall weakens as the outer concentric eyewall forms and eventually disappears with the intensification of the outer eyewall. In fact, as indicated by Figs. 3.7 and 3.11, the inner eyewall starts to weaken long before the secondary tangential wind maximum forms. Although 
intensely studied, there are still debates on what causes the demise of the primary eyewall. Willoughby et al. $(1982 ; 1990)$ argued that the outer eyewall could induce substantial subsidence over the inner eyewall region to inhibit the eyewall convection and eventually lead to the demise of the inner eyewall. This argument is questioned by Rozoff et al. (2008) who derived an analytical solution of transverse circulation equation of a balanced vortex model and showed that the subsidence induced by the outer eyewall is primarily within the eye and the moat. The simulation and Sawyer-Eliassen diagnoses performed in this study allow us to revisit this issue using the realistically simulated numerical data of a TC vortex by a 3D full physics model.

The decomposition of total heating into the eyewall and outer rainband components performed previously provides a useful way to examine the interaction between the eyewall and outer rainband convection during ERC. Figure3.21 shows the subsidence induced by the outer rainband heating overlapped with the WRF simulated azimuthalmean vertical velocity at different time during the ERC. The outer rainband induced subsidence contributes significantly to the moat and the strongest subsidence is at the outer edge of rainband updraft, which is typical for the convection induced subsidence. However, the figure clearly shows that the outer rainband induced subsidence is nonnegligible right over the inner eyewall updraft at all time. As indicated by Figs. 3.11a, $3.11 \mathrm{~b}$, and $3.11 \mathrm{c}$, the inner eyewall starts to weaken well before the outer concentric eyewall forms. Apparently, the rainband induced subsidence is one of reasons to cause such weakening of inner eyewall. This mechanism is enhanced with the increase of subsidence as the outer rainbands evolve into a closed ring of convection. This result pro- 
vides the convincing evidence to support Willoughby et al. (1982;1990)'s argument about the role of downdraft induced by the outer eyewall in causing the demise of the inner eyewall. But it should be emphasized here that the weakening of inner eyewall starts well before the formation of the outer eyewall. The suddenly enhanced outer rainband convection can induce sufficiently strong subsidence to affect the evolution of inner eyewall.

Figure3.22 is a similar plot to Figure 3.21 but for the subsidence induced by the inner eyewall heating. Similar to the subsidence induced by the outer rainband heating, the strongest subsidence occurs at the outer edge of the eyewall updraft. It is the leading cause for the formation and development of moat. The eyewall heating also induces fairly strong subsidence over the outer rainband but its influence decreases with the weakening of inner eyewall as the outer eyewall develops. An immediate question is why the outer rainband induced subsidence can inhibit the development of inner eyewall whereas the inner eyewall induced subsidence does not appear to have a significant impact on the development of the outer rainbands. The answer may lie in the radial inflow induced by the heating and momentum forcing. Figure 3.23 shows the radial flow induced by the eyewall and outer rainband heating and momentum forcing, respectively. Both eyewall and outer rainband forcings induce a similar structure of radial flow with outflow concentrated within the eyewall and rainband updraft and inflow extending radially outward from the outer edge of outflow. Before the $72^{\text {nd }} \mathrm{h}$ when outer rainband convection is weak, the eyewall induced radial inflow extends well beyond the "SEF region". The moist air carried by the radial inflow provides fuel for the development of eyewall. However, the outflow induced by the outer rainband forcing provides a mechanism to cancel 
the inflow induced by the eyewall. The cancellation increases as the outer rainband convection enhances and the eyewall weakens possibly due to the reduced inflow. By the time the outer eyewall forms $\left(78^{\text {th }} \mathrm{h}\right)$, its induced radial outflow extends throughout the vertical column, which works as a barrier to cut off the radial inflow into the inner eyewall, and indeed as the figures indicated the radial inflow induced by the inner eyewall at this time is really confined between the inner and outer eyewall. The cutting off fuel to the inner eyewall causes it to rapidly decay and eventually disappear. Again, the importance of enhanced rainband convection to ERC is clearly demonstrated from the perspective of transverse circulation. In short, the analyses here along with the evidence provided suggest a mechanism for the demise of the inner eyewall that involves the interaction between the transverse circulations induced by the eyewall and outer rainband convection. In particular, the outflow induced by the outer rainbands plays an important role in confining the inflow induced by the eyewall. The cutting off fuel into the inner eyewall appears to be the leading cause for the rapid decay of the inner eyewall.

\subsubsection{Propagation of outer rainband convection during ERC}

Figure 3.4 suggests a radially inward propagation of outer rainbands during ERC. Although frequently observed, the underlying mechanism that governs the propagation of outer rainbands is not well addressed. To look into this issue, we treat eyewall and outer rainband convection as asymmetric PV perturbation superimposed on a symmetric PV field and use the classic Fourier transform to decompose the asymmetric PV perturbations in terms of wavenumbers. Since total energy is conserved under the Fourier transform, the Fourier power spectrum may be used to measure the relative contribution of 
different wavenumber PV perturbations to the total energy of asymmetric perturbations. To do so, we define a normalized power spectrum (NPS), $E(r, n)$, as,

$$
E(r, n)=\frac{\sum_{n=1}^{N-1}|c(r, n)|^{2}}{\max \left(\sigma(r)^{2}\right)},
$$

where $n$ is the wavenumber, $c(r, n)$ is the Fourier transform coefficient at the radius $r$. $\sigma(r)^{2}$ is the variance of PV perturbation at the radius $r . N=180$ is the maximum wavenumber allowed in the Fourier transform since there are 361 azimuthal points in the domain. Figs. 3.24 and 3.25 show the time-radius plots of NPS at the heights of $2 \mathrm{~km}$ and 5 $\mathrm{km}$ overlapped with the radial gradient of azimuthal-mean PV. Low-wavenumber (1-3) components dominate the perturbation energy in the eyewall region, whereas highwavenumber (>3) components dominate the outer rainband region. Wavenumber 1 and 2 components have comparable magnitudes in the eyewall region at both low and mid troposphere. This result appears different from the observational study by Reasor et.al (2000) who found that the wavenumber 2 perturbation prevails at low-level but wavenmber 1 component appears to dominate at mid- and high-troposphere. This discrepancy could be attributed to the fact that there is lack of vortex motion and environmental shear in our simulation, which have been suggested as the leading causes for wavenumber 1 perturbation (Chen et. al, 2006;Reasor et.al, 2000). Most importantly as the figures indicated, the eyewall PV perturbations do not propagate outside of the eyewall region marked by the blue and red contours (which indicate the values of PV radial gradient of $-5 \times 10^{-10}$ and $5 \times 10^{-10} \mathrm{~s}^{-1}$ respectively) and beyond that region the PV radial profile is really flat. According to the finding by Montgomery and Kallenback (1997), 
the outward propagation of vortex Rossby waves requires negative radial PV gradient. It is, thus, not surprise that few perturbations initiated in the eyewall propagate beyond the radius of $40 \mathrm{~km}$ since no sufficient $\mathrm{PV}$ radial gradient supports such propagation. This result is consistent with Judt and Chen (2010)'s simulation of Hurricane Rita (2005) and Sun et. al (2013)'s simulation of Typhoon Sinlaku (2008) in which very weak vortex Rossby wave activities were found outside the eyewall region.

Figs. 3.24 and 3.25 also clearly show that the outer rainband perturbations propagate radially inward with time. The fact that all wavenumber perturbations align nearly parallel to the constant PV radial gradient and that high wavenumber perturbations dominate the outer rainband perturbations indicates that the propagation of outer rainband perturbations must not be explained by the vortex Rossby waves. Willoughby (1978) showed that the inertial-buoyancy (IB) waves in an idealized symmetric TC vortex have an inward-propagating mode, which may be used to explain the inward propagation of outer rainband perturbations. However, some characteristics of IB waves predicted by this theory are not consistent with our simulations. For example, the IB wave theory predicts a substantial increase of frequency toward the vortex center, but such an increase in wave frequency with the decrease of radius is not shown in Figs. 3.24 and 3.25. How to appropriately explain the propagation of outer rainbands using wave theory remains to be issue that needs to be further investigated. Here we attempt to approach this issue in the same balanced symmetric framework based on the Sawyer-Eliassen equation.

SW82 showed that for most cases in their idealized experiments the eyewall diabatic heating induced acceleration of tangential wind is the largest just inside of the 
RMW leading to the contraction of RMW as the vortex intensifies. Since both eyewall and outer rainband diabatic heating are resulted from moist convection, it is interesting to see if SW82's finding can be extended and applied to outer rainbands. Figure 3.26 shows the outer rainband diabatic heating overlapped with its induced convergence of radial inflow and tendency of tangential wind. Both convergence of radial flow and acceleration of tangential wind show an interesting tilted vertical structure. The strongest convergence of radial flow is concentrated at the outer edge (radially) of rainband heating and tilted inward as it descends toward the surface. The largest acceleration of tangential wind is in the upper boundary layer and inside the main body of outer rainband heating. Such a structure favors the rainband convection moving inward in a similar manner of eyewall contraction proposed by SW82. However, it should be pointed out unlike eyewall contraction and ERC, which to the first approximation is a symmetric problem, and thus, may be appropriately addressed in the symmetric framework, the outer rainband convection is fundamentally an asymmetric problem. To fully understand the formation, development, and propagation of outer rainbands, further studies are needed. 


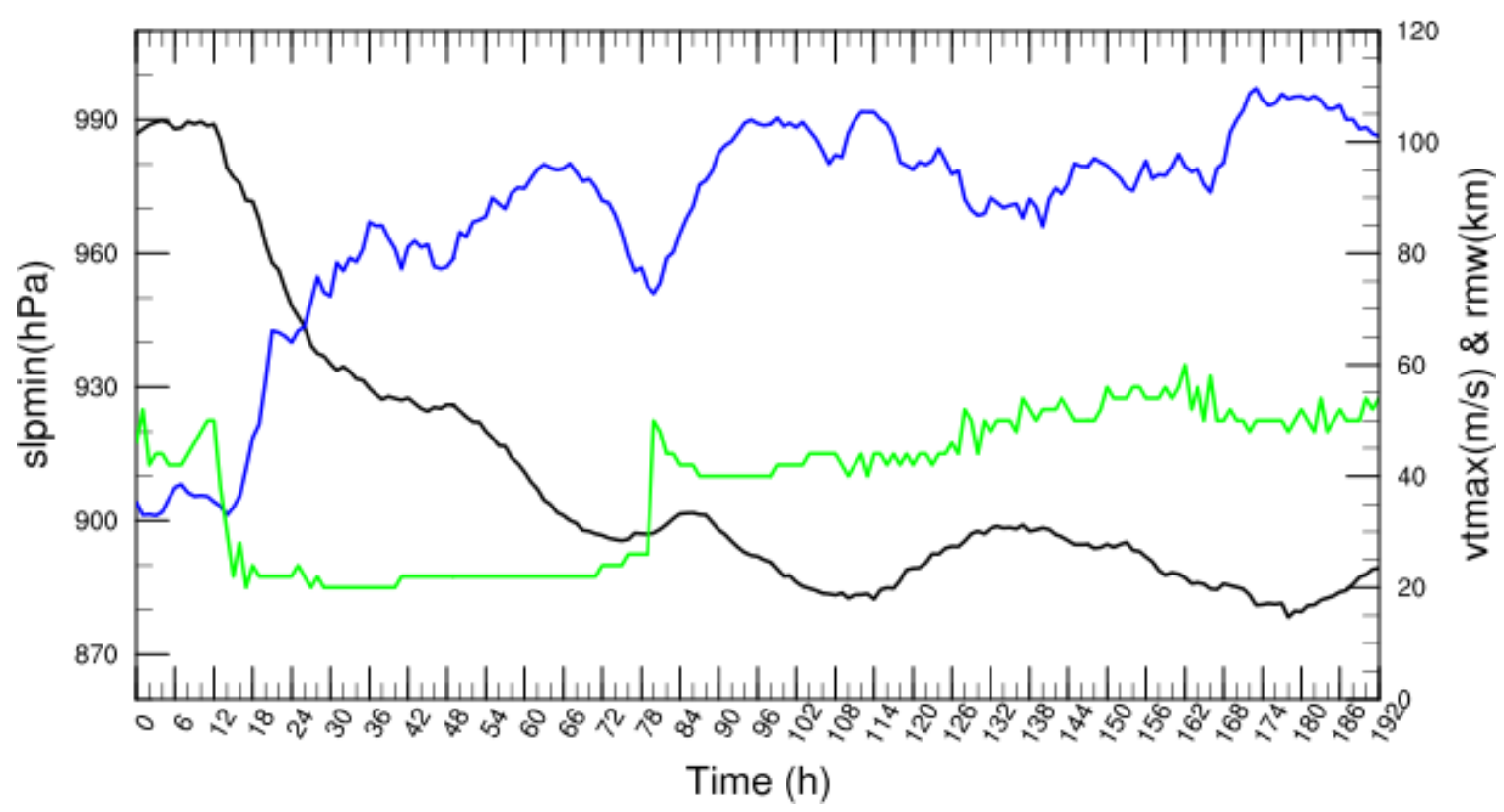

Figure 3.1 Time evolution of the minimum surface pressure (SLPMIN in hPa, black curve), the maximum mean tangential wind at $1 \mathrm{~km}$ (vtmax in $\mathrm{m} / \mathrm{s}$, blue curve), and the radius of maximum tangential wind (RMW in km, green curve). 


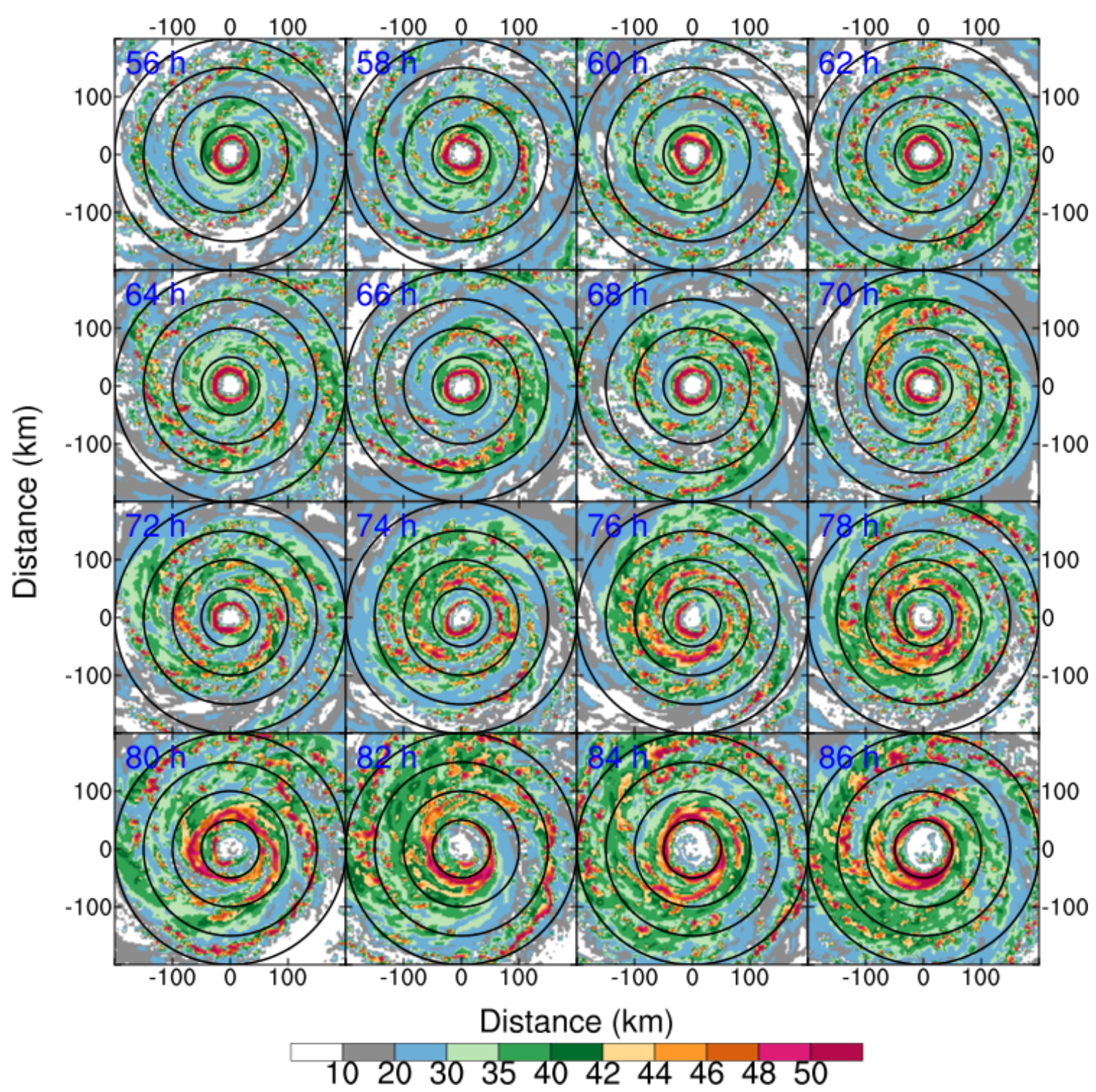

Figure 3.2 Snapshots of synthetic radar reflectivity (dBZ) at $1 \mathrm{~km}$ level from $56 \mathrm{~h}$ to

$86 \mathrm{~h}$ at a time interval of 2 hours. The black circles indicate $50 \mathrm{~km}, 100$

$\mathrm{km}, 150 \mathrm{~km}$ and $200 \mathrm{~km}$ radii. 

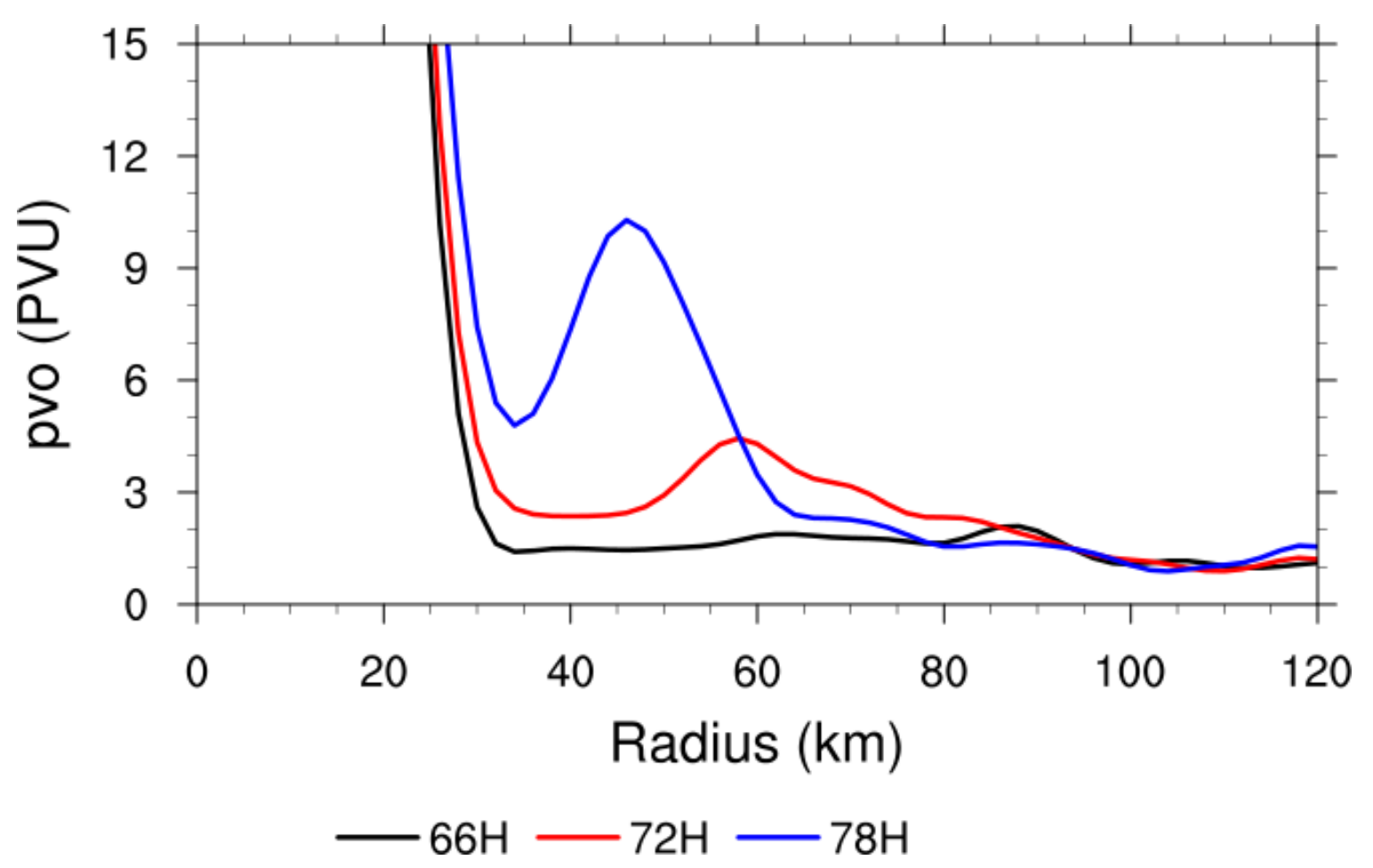

Figure 3.3 Radial profiles of azimuthal mean potential vorticity (smoothed with 5 passes of 1:2:1 smoother) at $1 \mathrm{~km}$ level between 66 and $78 \mathrm{~h}$ at a time interval of $6 \mathrm{~h}$. 


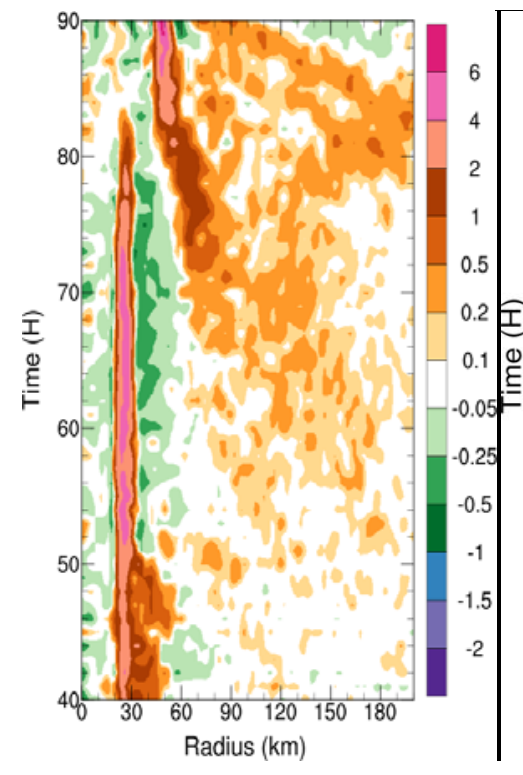

(a)

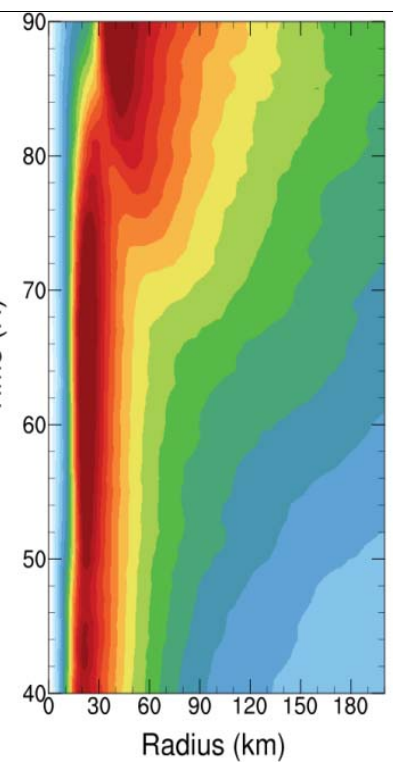

(b)

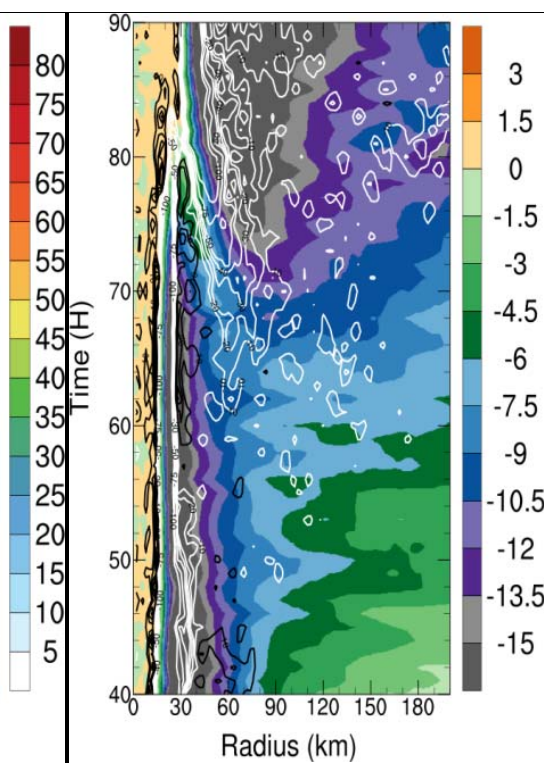

(c)

Figure 3.4 Time-radius plots of azimuthal-mean (a) vertical velocity $(\mathrm{m} / \mathrm{s})$ at $5 \mathrm{~km}$ level, (b) tangential wind $(\mathrm{m} / \mathrm{s})$ at $1000 \mathrm{~m}$ level, $(\mathrm{c})$ radial wind $(\mathrm{m} / \mathrm{s})$ at $500 \mathrm{~m}$ level. The contours in (c) are horizontal divergence at 500m level, with negative and positive values plotted in white and black colors, respectively. Contour intervals are 10 for values between -50 . and 50., and 25 for values above 50 . and below -50 . 

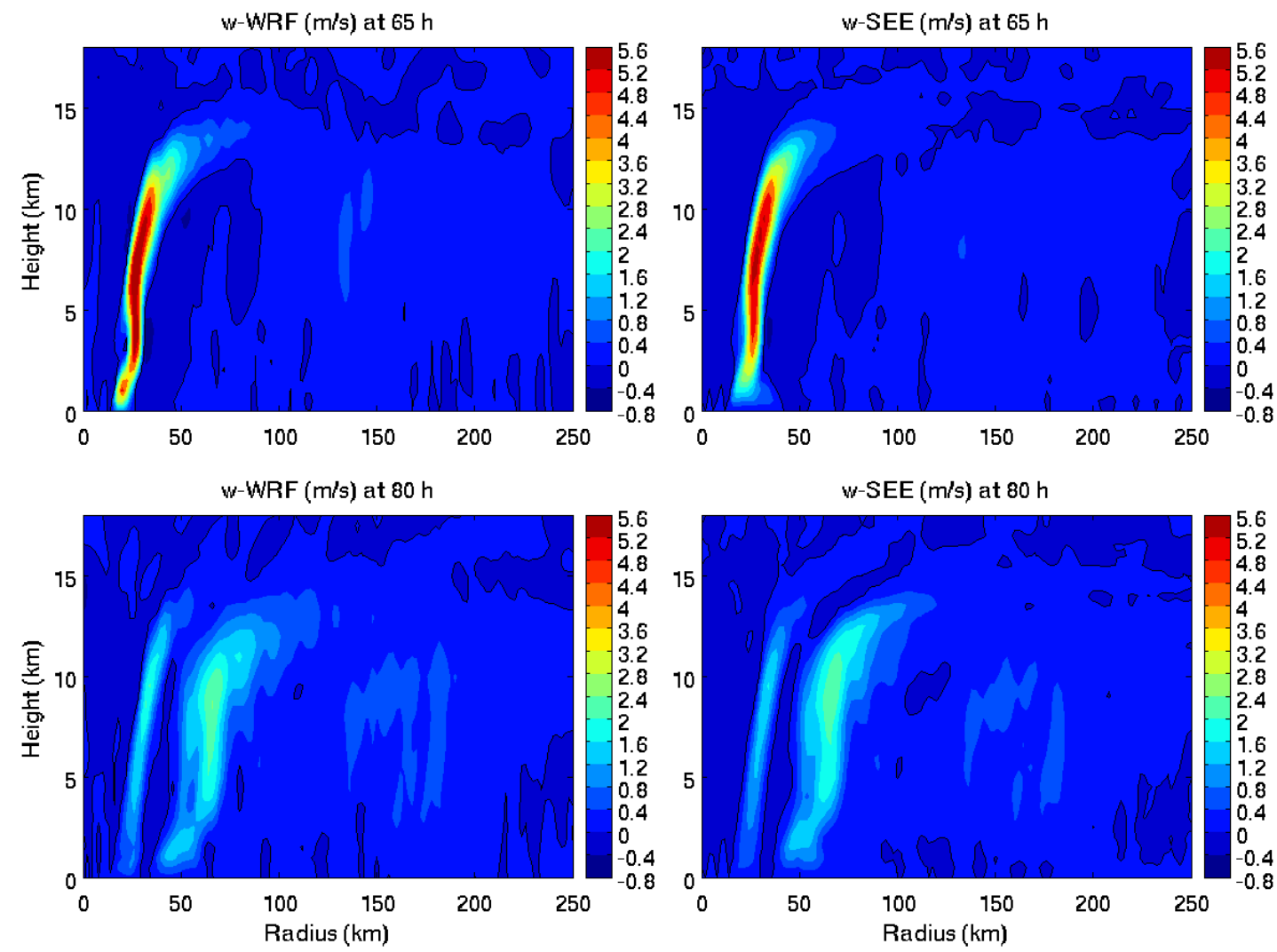

Figure 3.5 Azimuthal-mean vertical velocities simulated by WEF (left column) and those from the Sawyer-Eliassen diagnoses at the $65^{\text {th }} \mathrm{h}$ and $80^{\text {th }} \mathrm{h}$. Black contours in the figure indicate zero vertical velocities. 


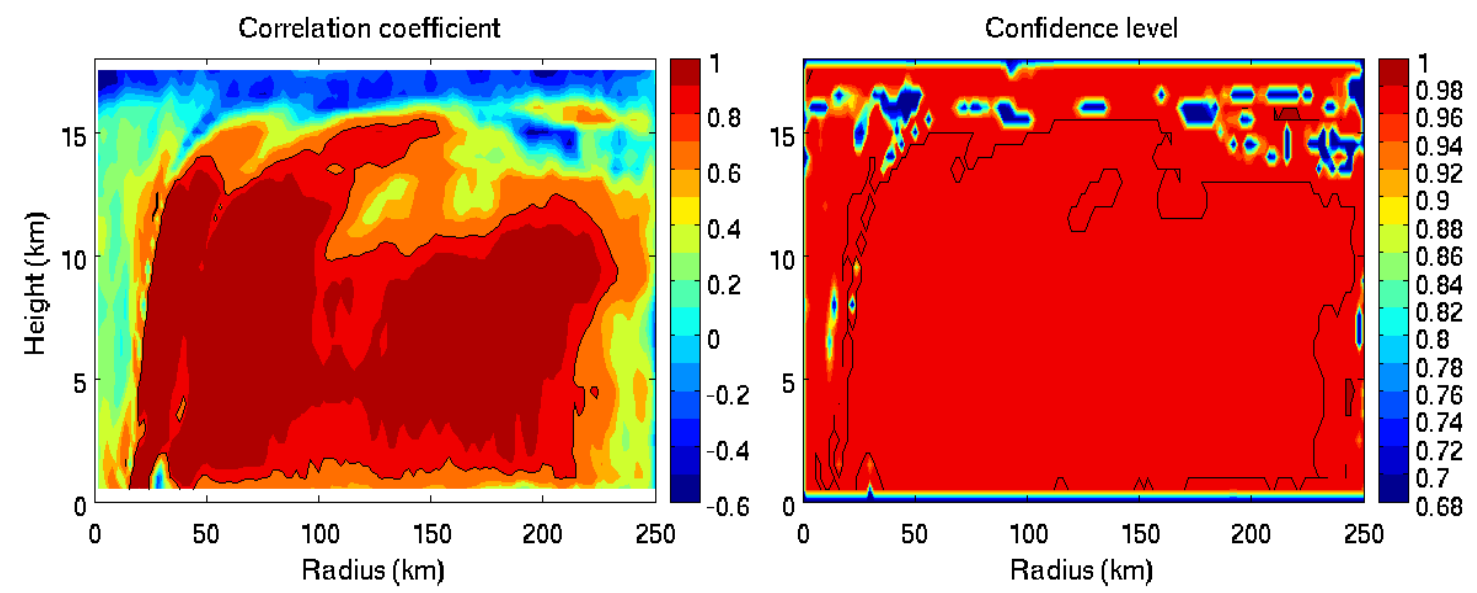

Figure 3.6 Correlation coefficient and the associated confidence level between the azimuthal-mean vertical velocities simulated by WRF and those from the Sawyer-Eliassen diagnoses calculated using the data from the $50^{\text {th }} \mathrm{h}$ to $90^{\text {th }}$ h. 
(a) time $=70 \mathrm{~h}$

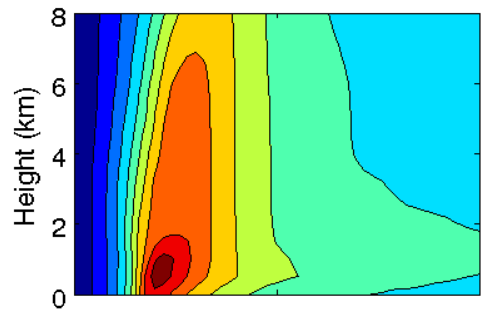

(d) time $=78 \mathrm{~h}$

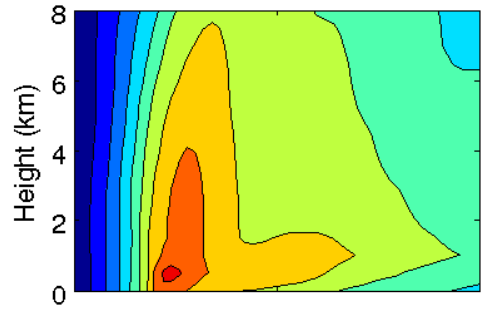

(g) time $=81 \mathrm{~h}$

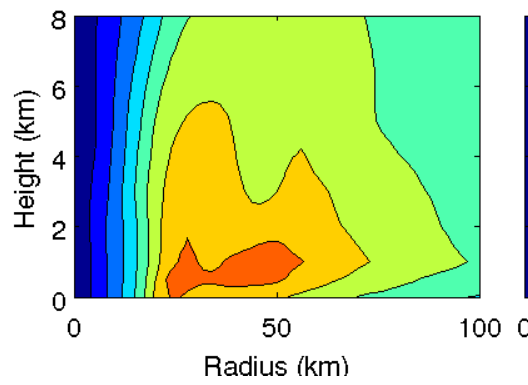

(b) time $=73 \mathrm{~h}$

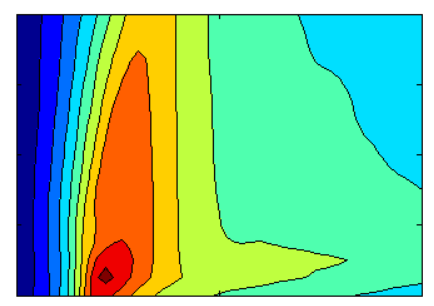

(e) time $=79 \mathrm{~h}$

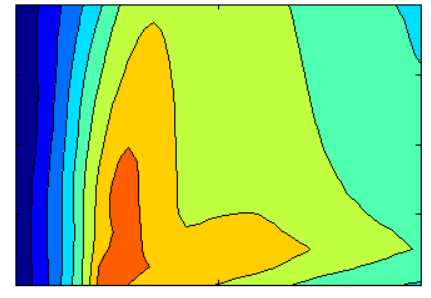

(h) time $=82 \mathrm{~h}$

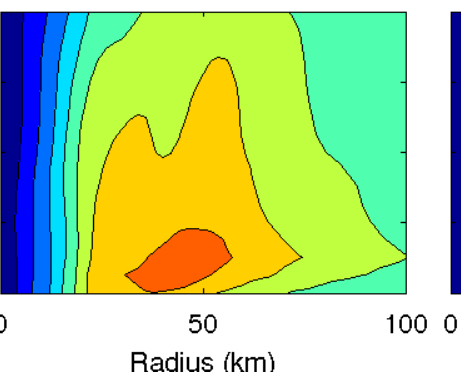

(c) time $=76 \mathrm{~h}$

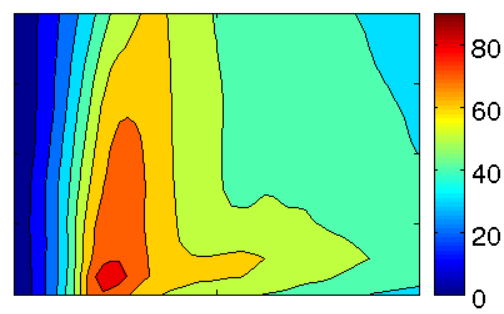

(f) time $=80 \mathrm{~h}$

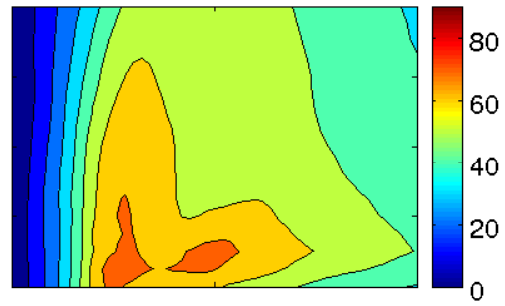

(i) time $=84 \mathrm{~h}$

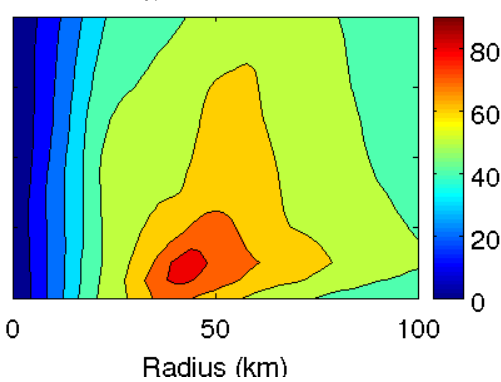

Figure 3.7 Time evolution of azimuthal-mean tangential wind simulated by WRF from the $70^{\text {th }} \mathrm{h}$ to $84^{\text {th }} \mathrm{h}$ 
(a) time $=70 \mathrm{~h}$

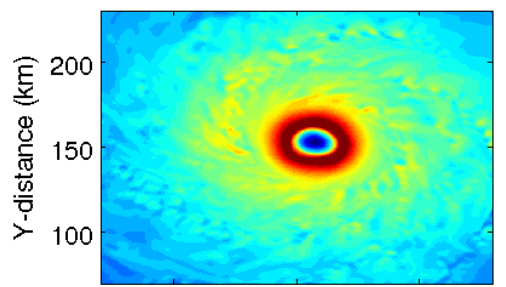

(d) time $=78 \mathrm{~h}$

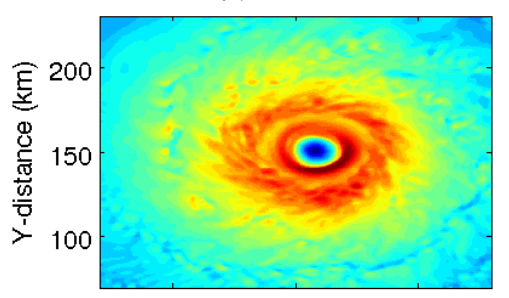

(g) time $=81 \mathrm{~h}$

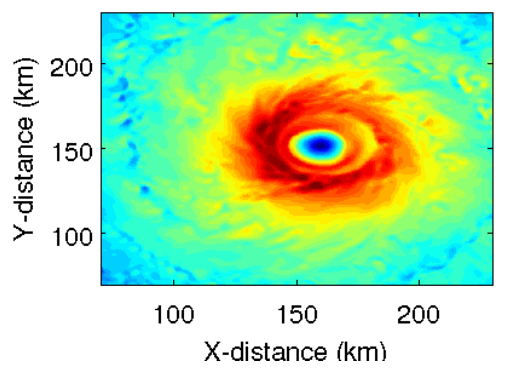

(b) time $=73 \mathrm{~h}$

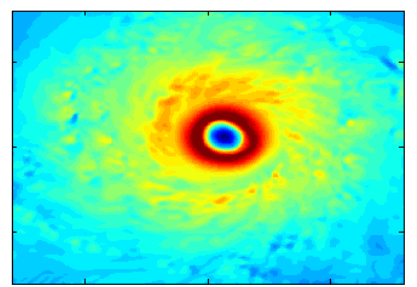

(e) time $=79 \mathrm{~h}$

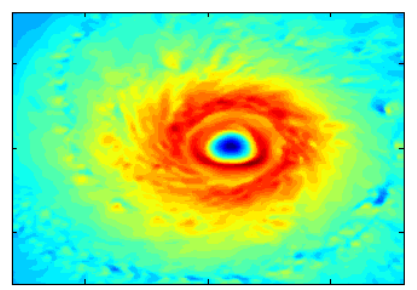

(h) time $=82 \mathrm{~h}$

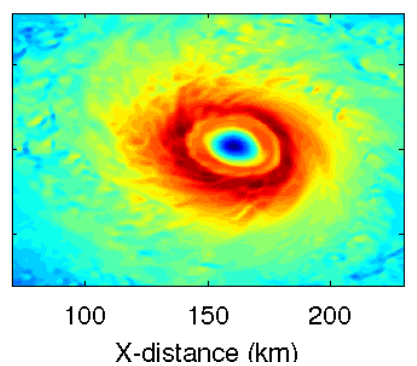

(c) time $=76 \mathrm{~h}$

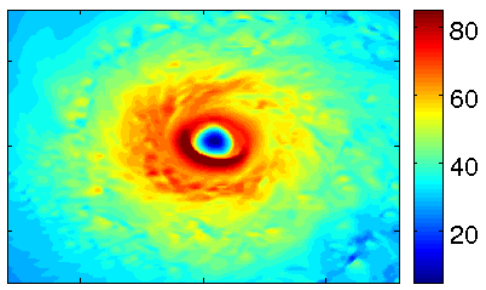

(f) time $=80 \mathrm{~h}$

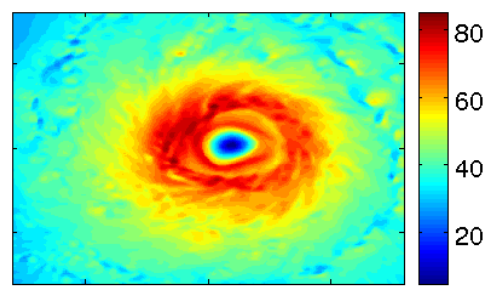

(i) time $=82 \mathrm{~h}$

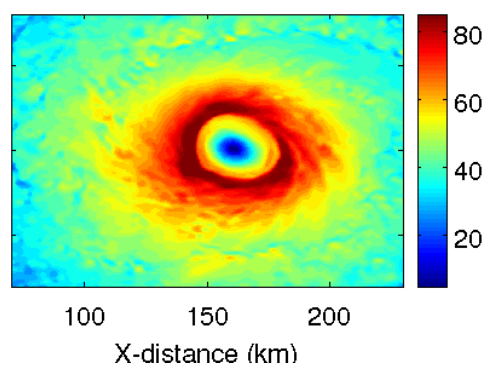

Figure 3.8 Time evolution of horizontal wind speed at $1 \mathrm{~km}$ simulated by WRF from the $70^{\text {th }} \mathrm{h}$ to $84^{\text {th }} \mathrm{h}$. 
(a) Net $\partial \bar{v} / \partial t(\times 10)$

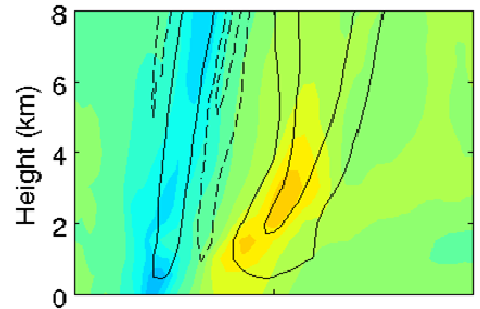

(d) $-\bar{u} \bar{\zeta}_{a}$

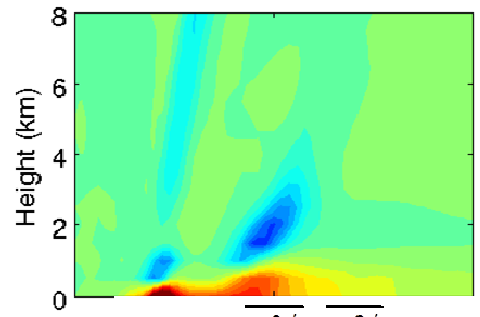

(g) $-\overline{u^{\prime} \xi_{\alpha}^{\prime}}-\overline{v^{\prime} \frac{\hat{v} v^{\prime}}{r O \partial}}-\overline{w^{\prime} \frac{\partial^{\prime}}{\partial z}}(\times 4)$

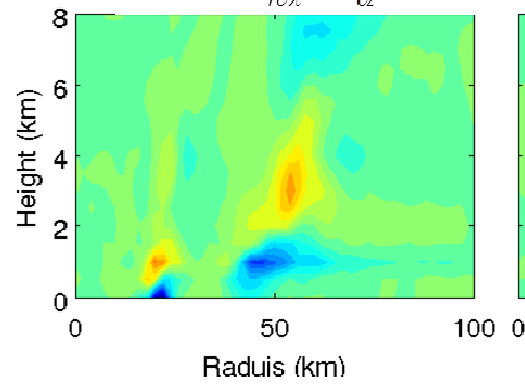

(b) $-\bar{u} \bar{\zeta}_{a}-\bar{w} \frac{\partial \bar{v}}{\partial z}-\overline{u^{\prime} \varsigma_{a}^{\prime}}-\overline{v^{\prime} \frac{v^{\prime}}{r \partial \lambda}}-\overline{w^{\prime} \frac{\partial v^{\prime}}{\partial z}}$

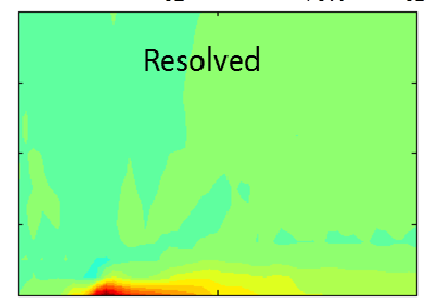

(e) $-\bar{w} \frac{\hat{v} v}{\partial z}$

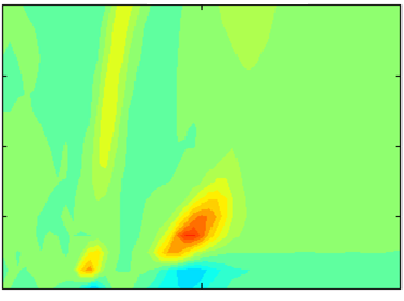

(h) SGS vertical diffusion

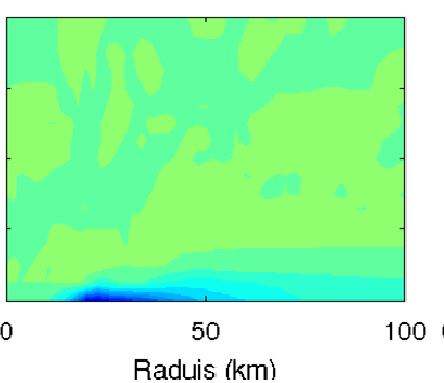

(c) $\overline{T S G S_{v}}$

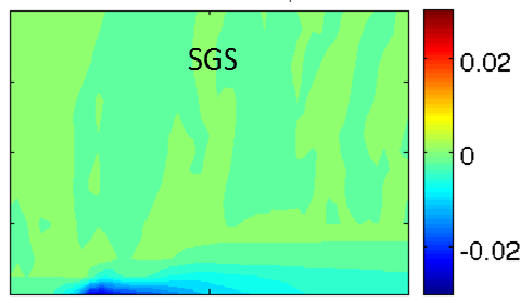

(f) $-\bar{u} \bar{\zeta}_{a}-\bar{w} \frac{\hat{v} \bar{v}}{\hat{\partial} z}$

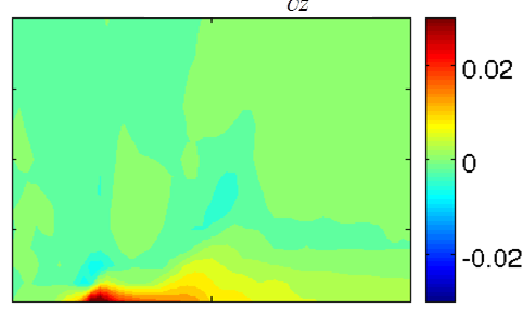

(i) SGS horizontal diffusion (X 20)

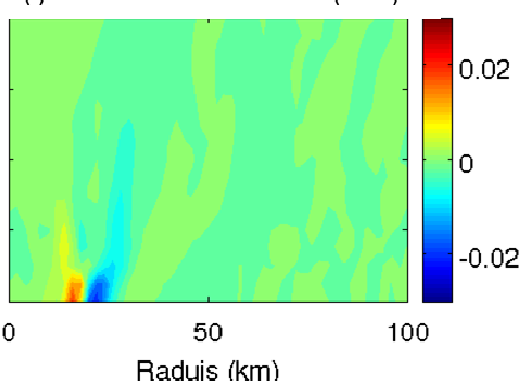

Figure 3.9 Tangential wind budget analyses averaged over the period from the $79^{\text {th }} \mathrm{h}$ to $81^{\text {st }}$ h. (a): Net tendency from the sum of all terms. Black solid and dashed contours indicate the up- and down-drafts. It has been multiplied by a factor of 10 ; (b): Tendency from the sum of all resolved terms (i.e., $\left.-\bar{u} \bar{\zeta}_{a}-\bar{w} \frac{\partial \bar{v}}{\partial z}-\overline{u^{\prime} \varsigma_{a}^{\prime}}-\overline{v^{\prime} \frac{\partial v^{\prime}}{r \partial \lambda}}-\overline{w^{\prime} \frac{\partial v^{\prime}}{\partial z}}\right) ;(\mathrm{c}):$ Tendency from the SGS processes; (d): Tendency from $-\bar{u} \bar{\zeta}_{a} ;(\mathrm{e}):$ Tendency from $-\bar{w} \frac{\partial \bar{v}}{\partial z} ;(\mathrm{f})$ : Tendency from $-\bar{u} \bar{\zeta}_{a}-\bar{w} \frac{\partial \bar{v}}{\partial z} ;(\mathrm{g})$ Tendency from the resolved asymmetric 
eddies, $-\overline{u^{\prime} \varsigma_{a}^{\prime}}-\overline{v^{\prime} \frac{\partial v^{\prime}}{r \partial \lambda}}-\overline{w^{\prime} \frac{\partial v^{\prime}}{\partial z}}$. It has been multiplied by a factor of 4 ; (h): Tendency from the SGS vertical turbulent mixing; (i): Tendency from the SGS horizontal turbulent mixing. It has been multiplied by a factor of 20 . 
(a) Mean tendency $(\times 1000)$ at $500 \mathrm{~m}$ over $79-81 \mathrm{~h}$

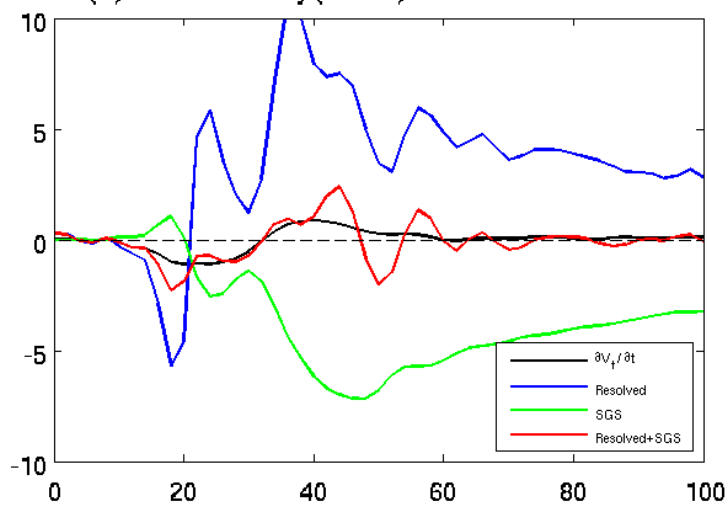

(c) Mean tendency $(\times 1000)$ at $1000 \mathrm{~m}$ over $79-81 \mathrm{~h}$

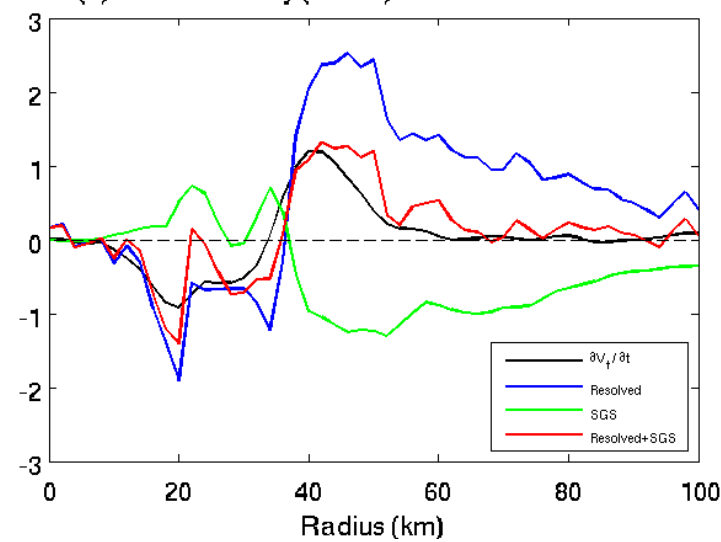

(b) Mean resolved tendency $\left(X_{1000)}\right.$ at $500 \mathrm{~m}$ over $79-81 \mathrm{~h}$

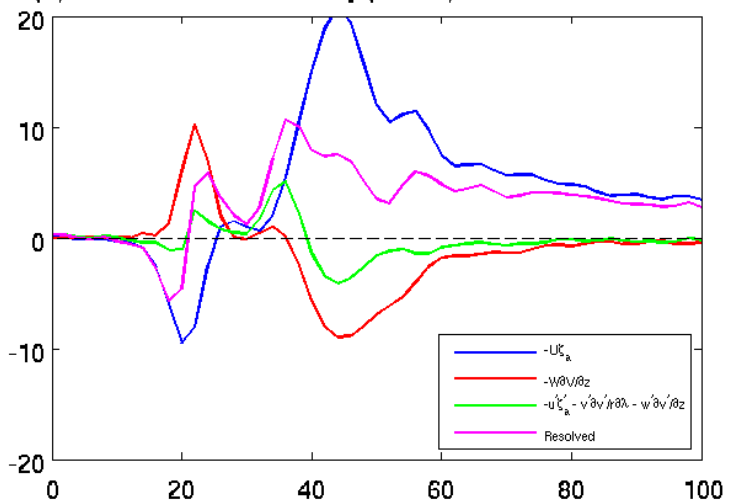

(d) Mean resolved tendency $(\times 1000)$ at $1000 \mathrm{~m}$ over $79-81 \mathrm{~h}$

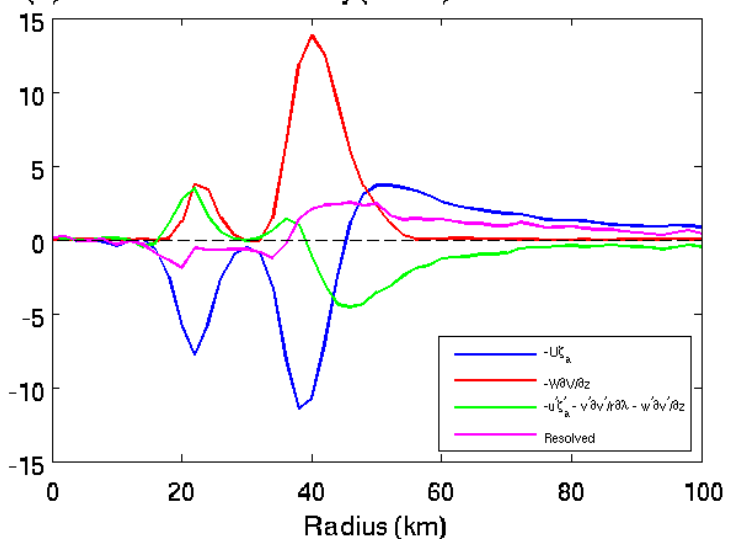

Figure 3.10 The radial profiles of tangential wind budget shown in Figure 3.8 at 500 $\mathrm{m}$ and $1000 \mathrm{~m}$, respectively. 
(a) Heating $(\mathrm{K} / \mathrm{s}) \times 1000$ at $50 \mathrm{~h}$

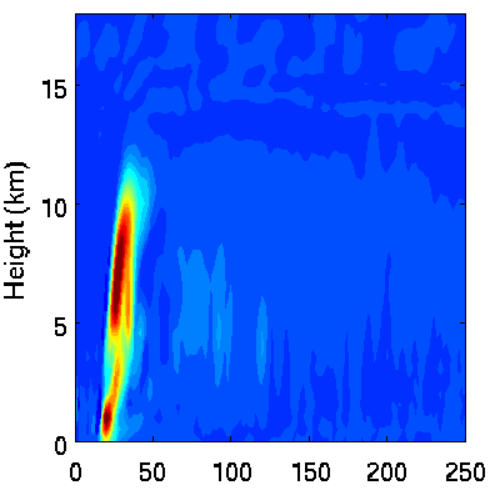

(d) Heating $(\mathrm{K} / \mathrm{s}) \times 1000$ at $76 \mathrm{~h}$

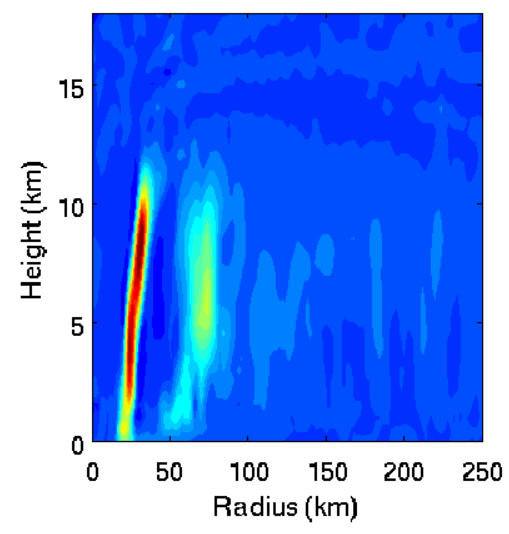

(b) Heating (K/s) $\times 1000$ at $72 \mathrm{~h}$

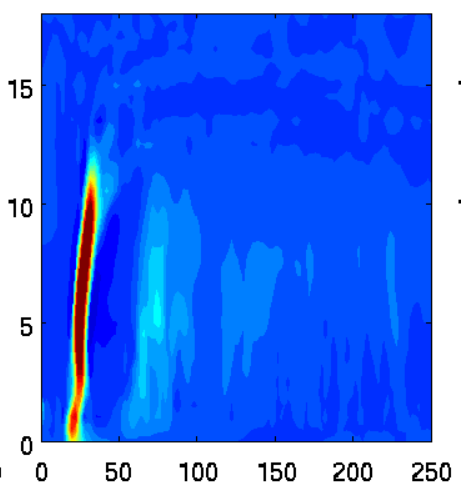

(e) Heating $(\mathrm{K} / \mathrm{s}) \times 1000$ at $78 \mathrm{~h}$

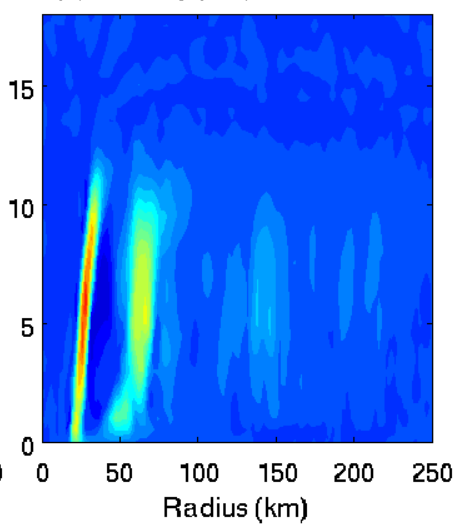

(c) Heating $(\mathrm{K} / \mathrm{s}) \times 1000$ at $74 \mathrm{~h}$

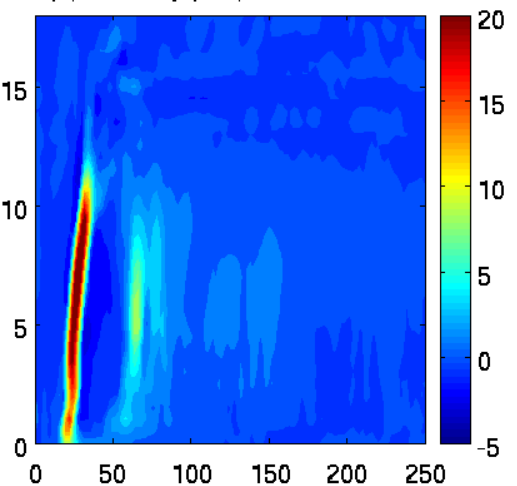

(f) Heating $(\mathrm{K} / \mathrm{s}) \times 1000$ at $80 \mathrm{~h}$

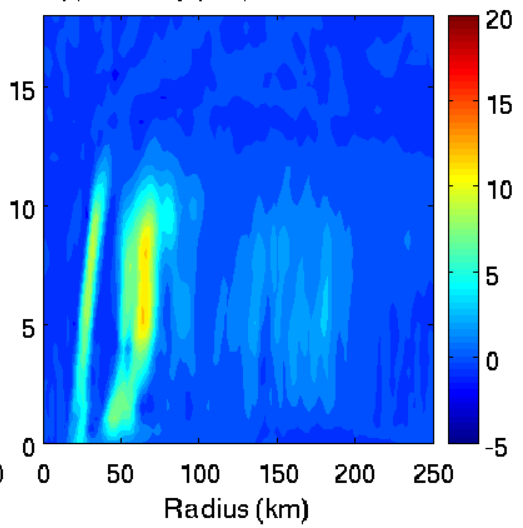

Figure 3.11 Azimuthal-mean diabatic heating derived from the WRF simulations at different time from the $50^{\text {th }} \mathrm{h}$ to $80^{\text {th }} \mathrm{h}$. 


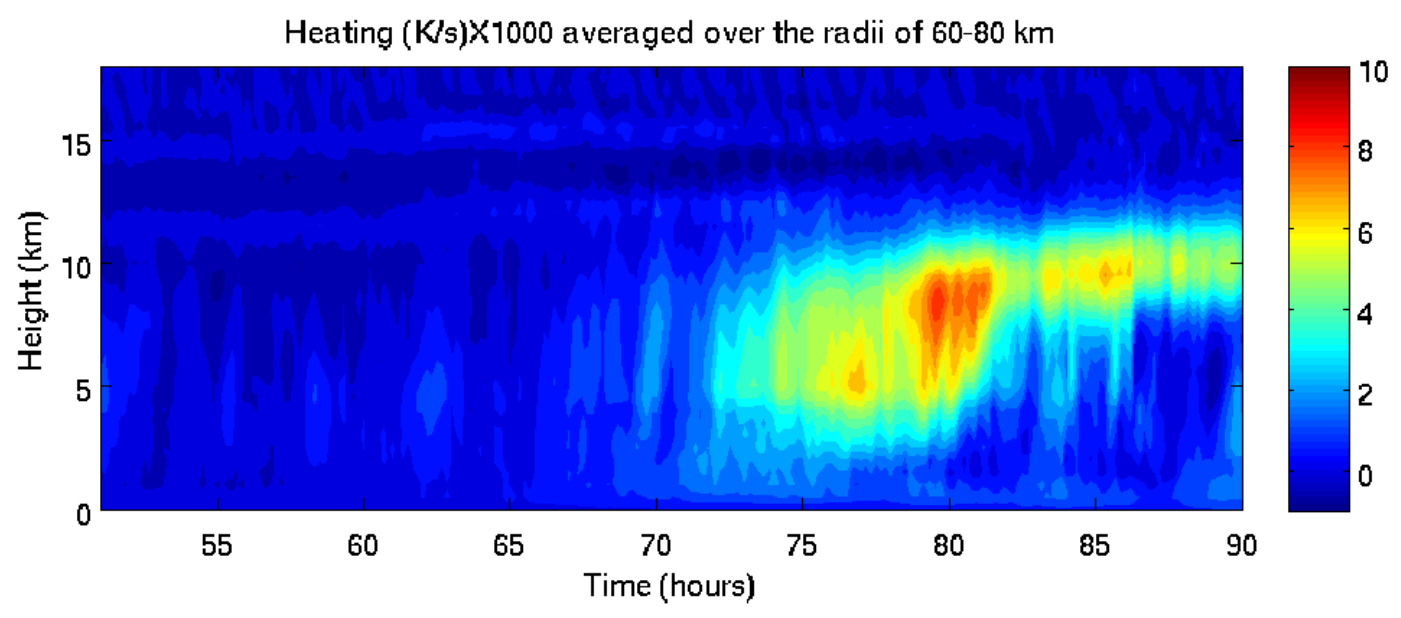

Figure 3.12 Time-height variation of daibatic heating averaged over the radii of 60-80 $\mathrm{km}$. 

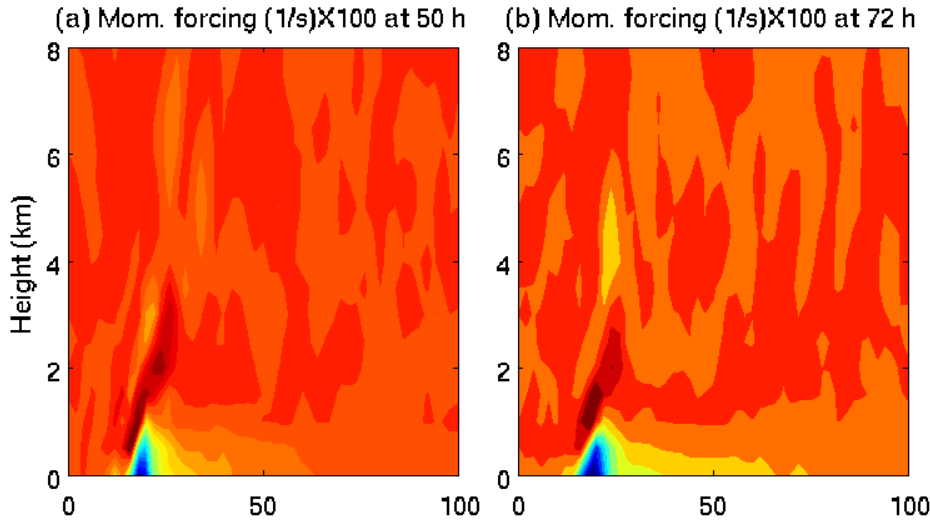

(c) Mom. forcing (1/s) $\times 100$ at $74 \mathrm{~h}$

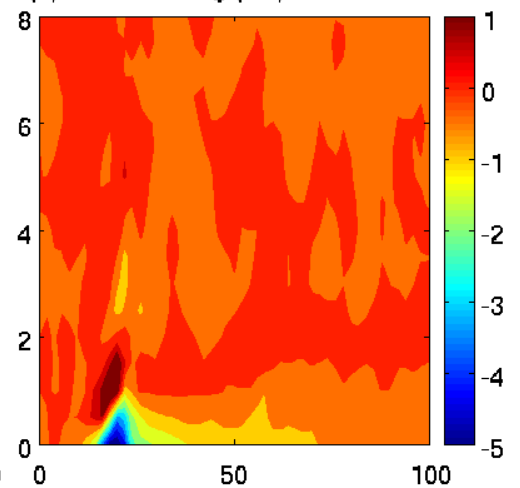

(d) Mom. forcing $(1 / \mathrm{s}) \times 100$ at $76 \mathrm{~h}$

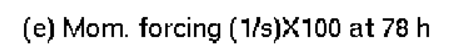

(f) Mom. forcing (1/s) $\times 100$ at $80 \mathrm{~h}$
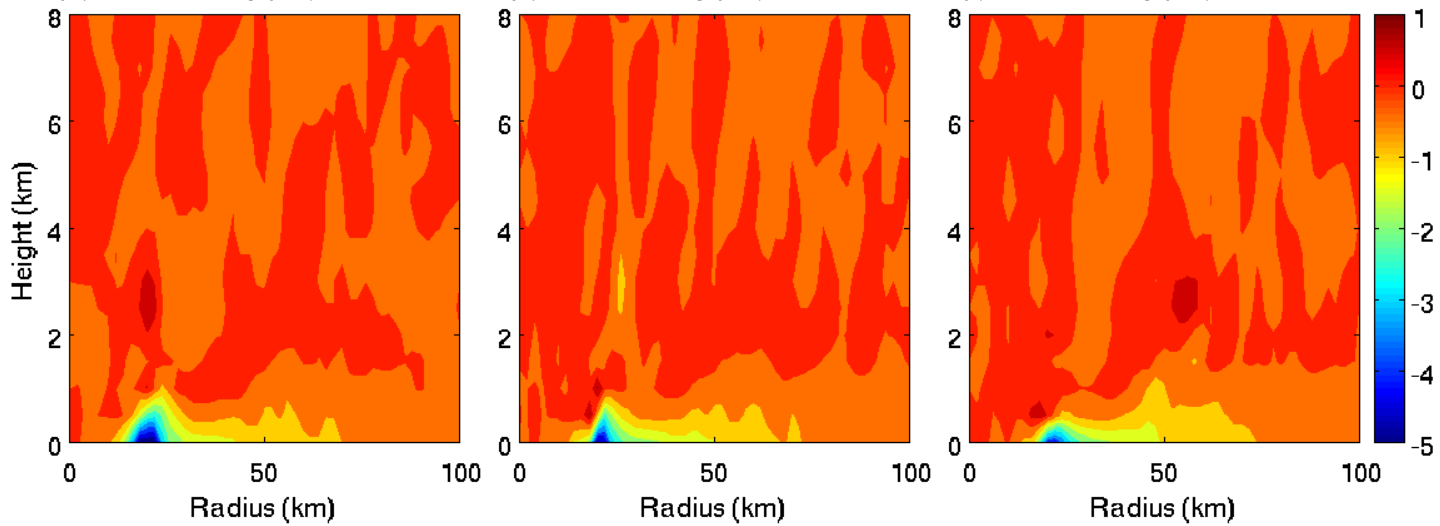

Figure 3.13 Momentum forcing derived from the WRF simulation from $50^{\text {th }} \mathrm{h}$ to $80^{\text {th }}$ h. 


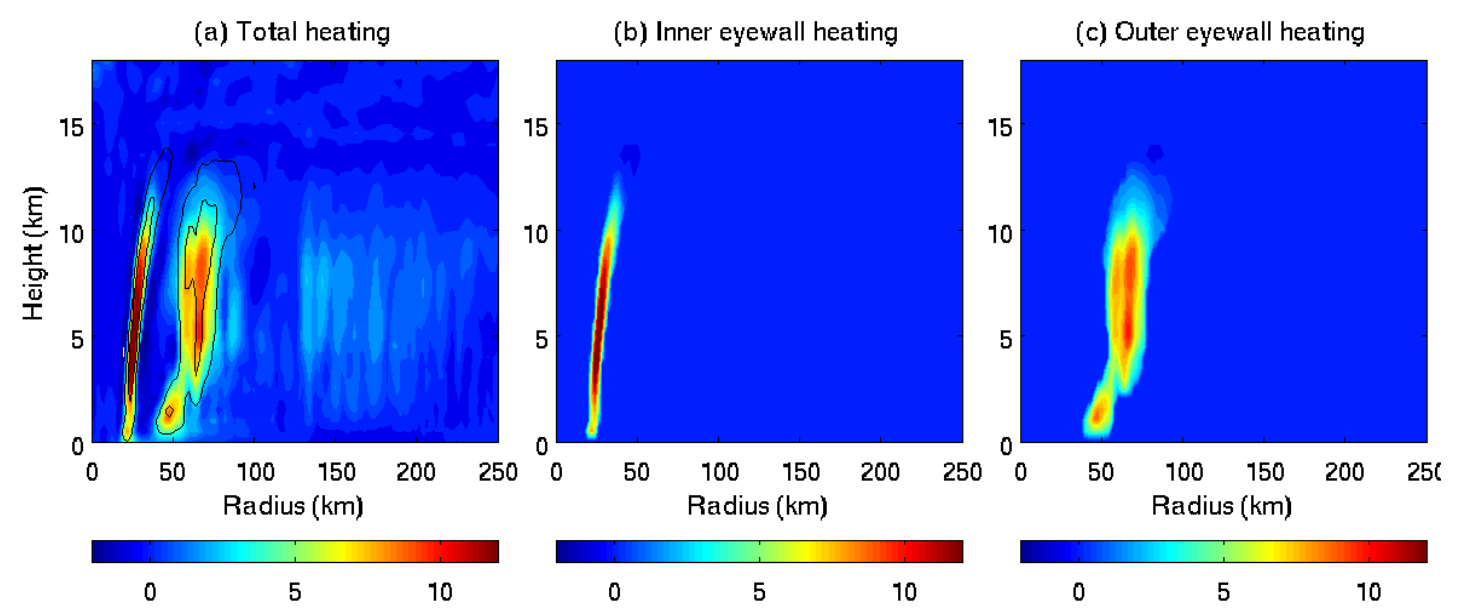

Figure 3.14 (a) Total diabatic heating at the $79^{\text {th }}$ h. (b): Eyewall diabatic heating with vertical velocity greater than $0.5 \mathrm{~m} / \mathrm{s}$. (c): Outer rainband diabatic heating with vertical velocity greater than $0.5 \mathrm{~m} / \mathrm{s}$. 

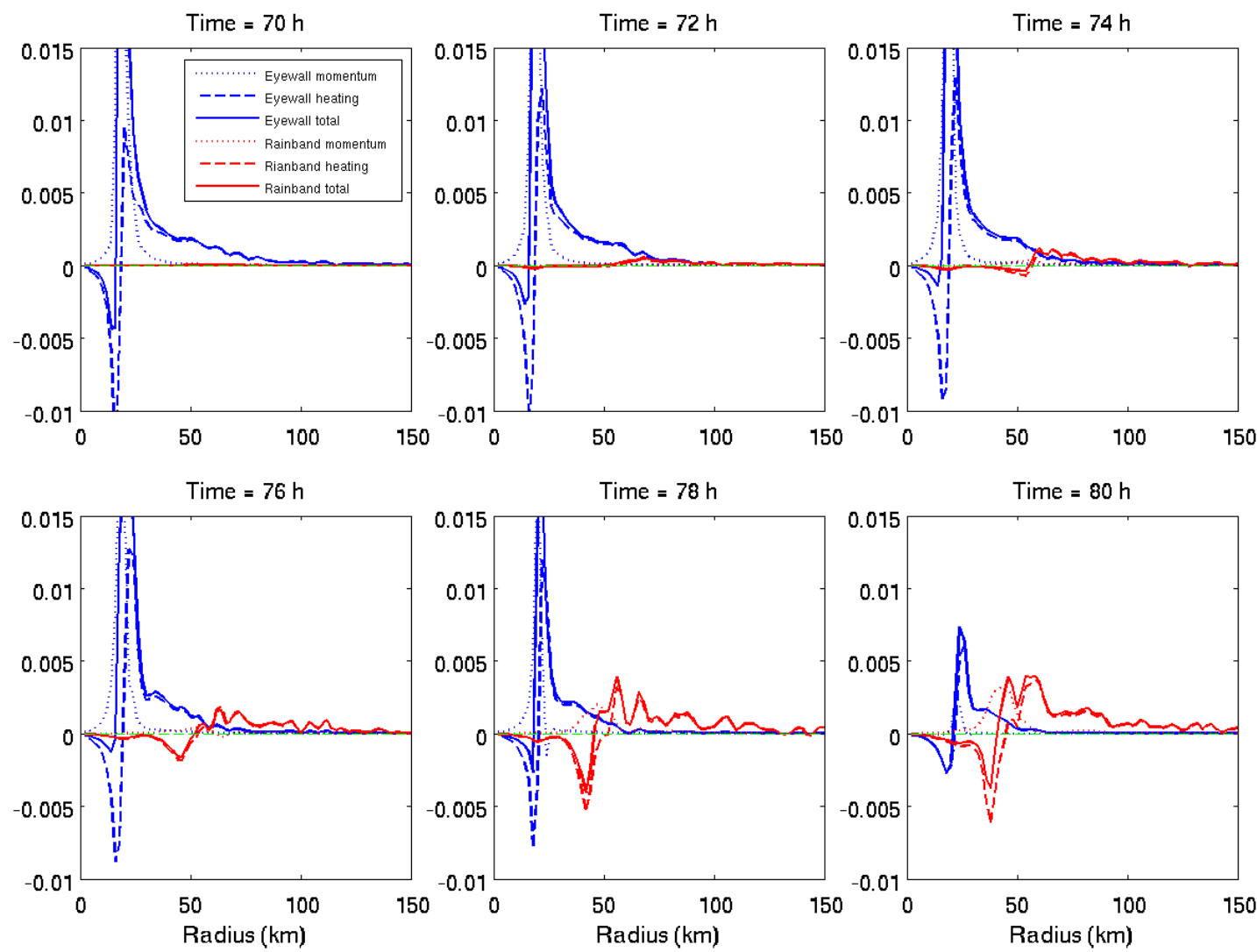

Figure3.15 Tendencies of tangential wind at $500 \mathrm{~m}$ induced by eyewall and outer rainbands derived from the Sawyer-Eliassen diagnoses indicated by the blue and red colors, respectively. The tendencies induced by the diabatic heating are indicated by the dashed curves and the tendencies induced by the momentum forcings are indicated by the dotted curves. 

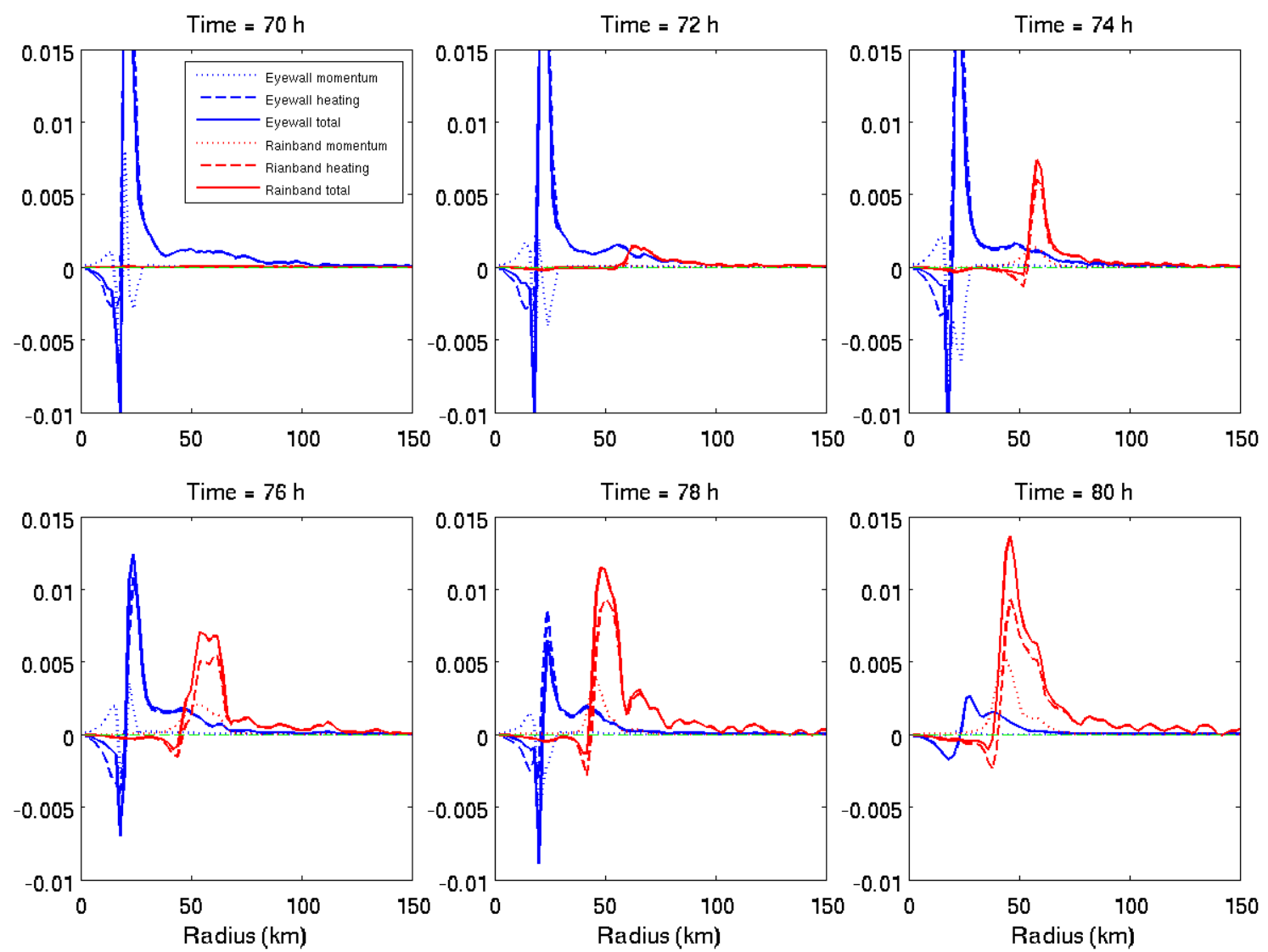

Figure 3.16 The same as Figure 3.15 but for the height of $1000 \mathrm{~m}$. 
(a) $\left.\left(\partial V_{t} / \partial t\right)_{\text {rainband }}-\partial V_{t} / \partial t\right)_{\text {eyewall }}$ averaged over the radii of $50-80 \mathrm{~km}$

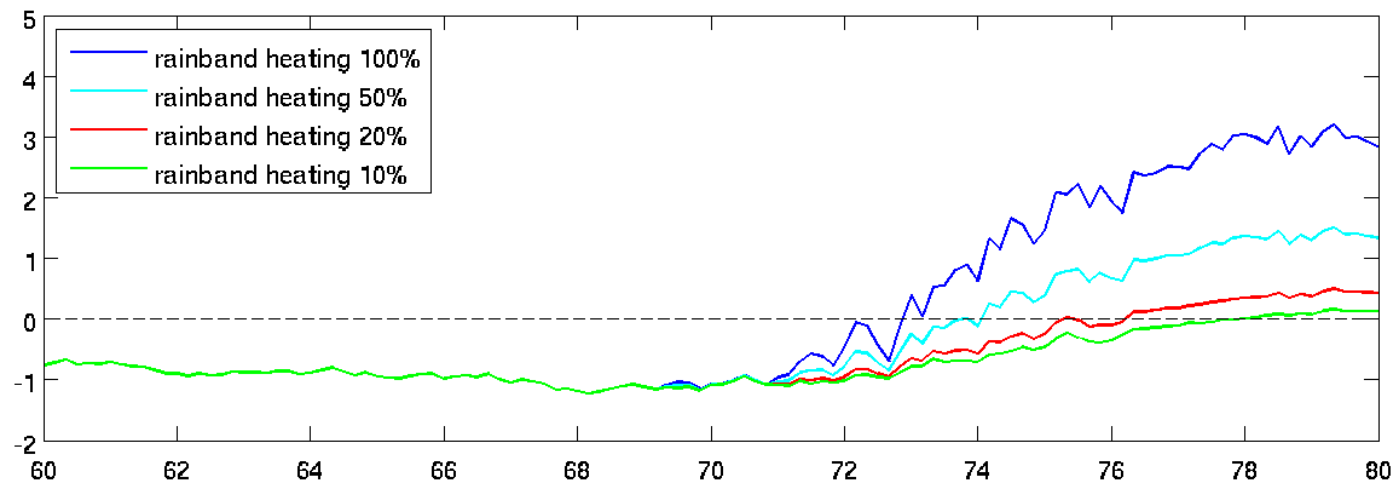

(b) Ratio of the strongest rainband heating to the strongest eyewall heating

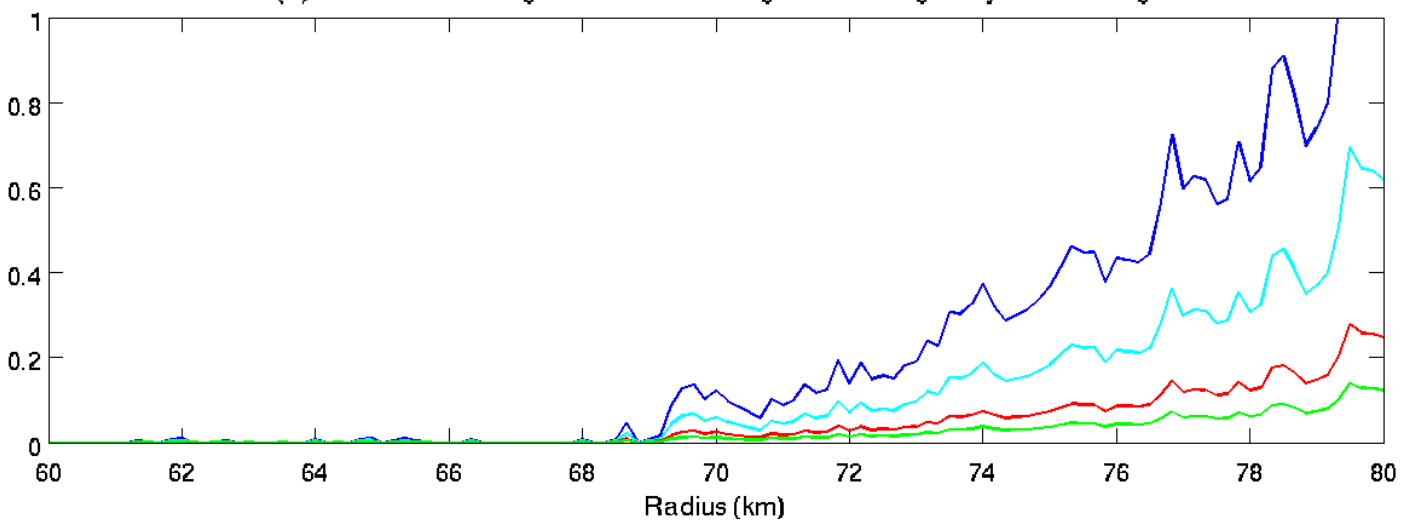

Figure 3.17 (a) Tendencies of tangential wind induced by the outer rainband heating with different strength (indicated by the different colors) averaged over the radii of 50-70 km subtracted by the corresponding tendency induced by the eyewall heating. (b): Ratio of the strongest outer rainband heating to the strongest eyewall heating for different experiments. 

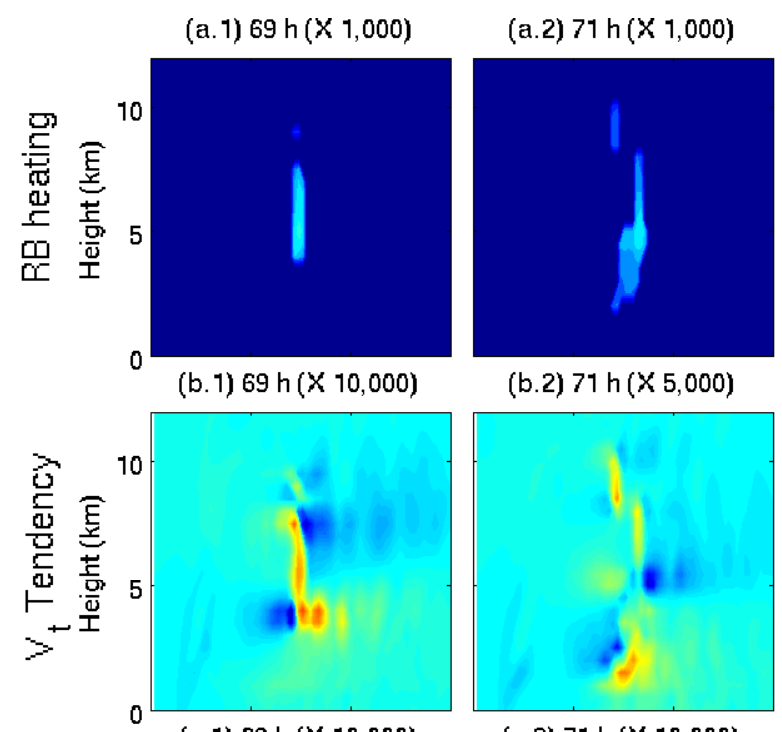

(b.2) $71 \mathrm{~h}(\times 5,000)$

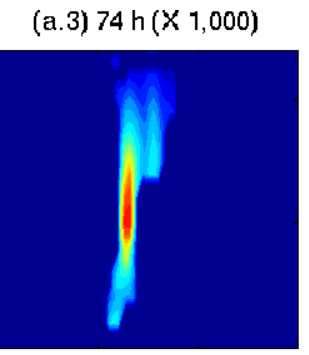

(b.3) $74 \mathrm{~h}(\times 2,000)$
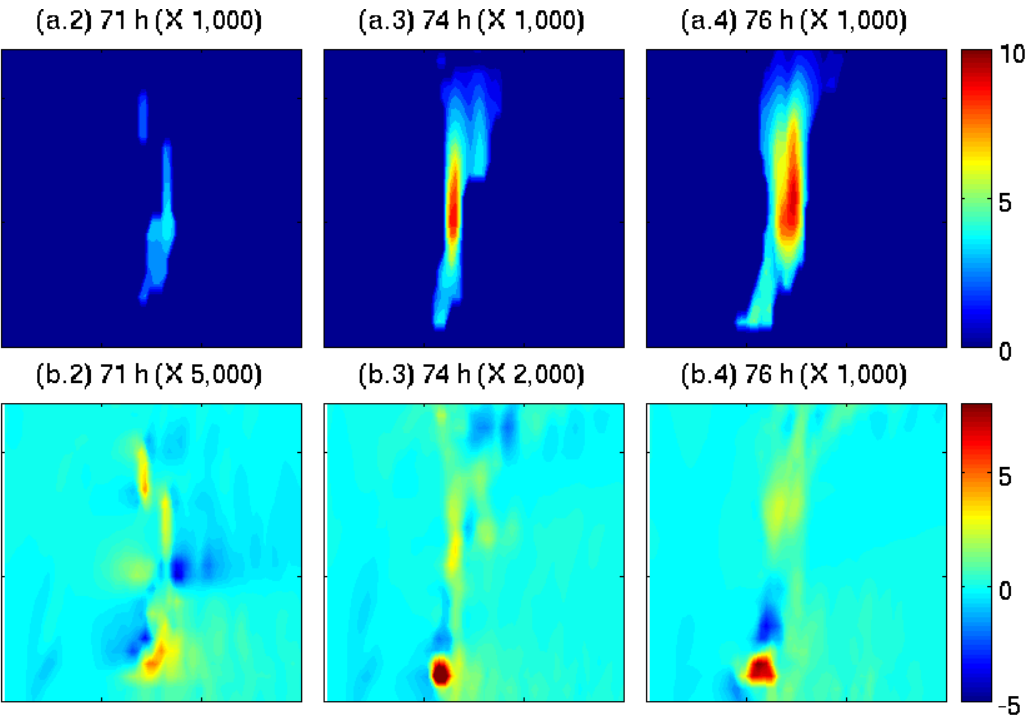

(c. 2$) 71 \mathrm{~h}(\times 10,000)$

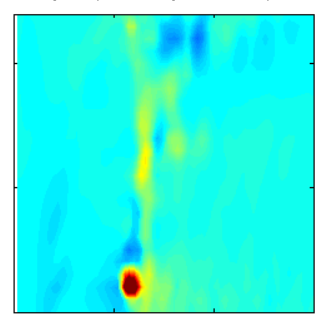

(c.3) $74 h(\times 10,000)$
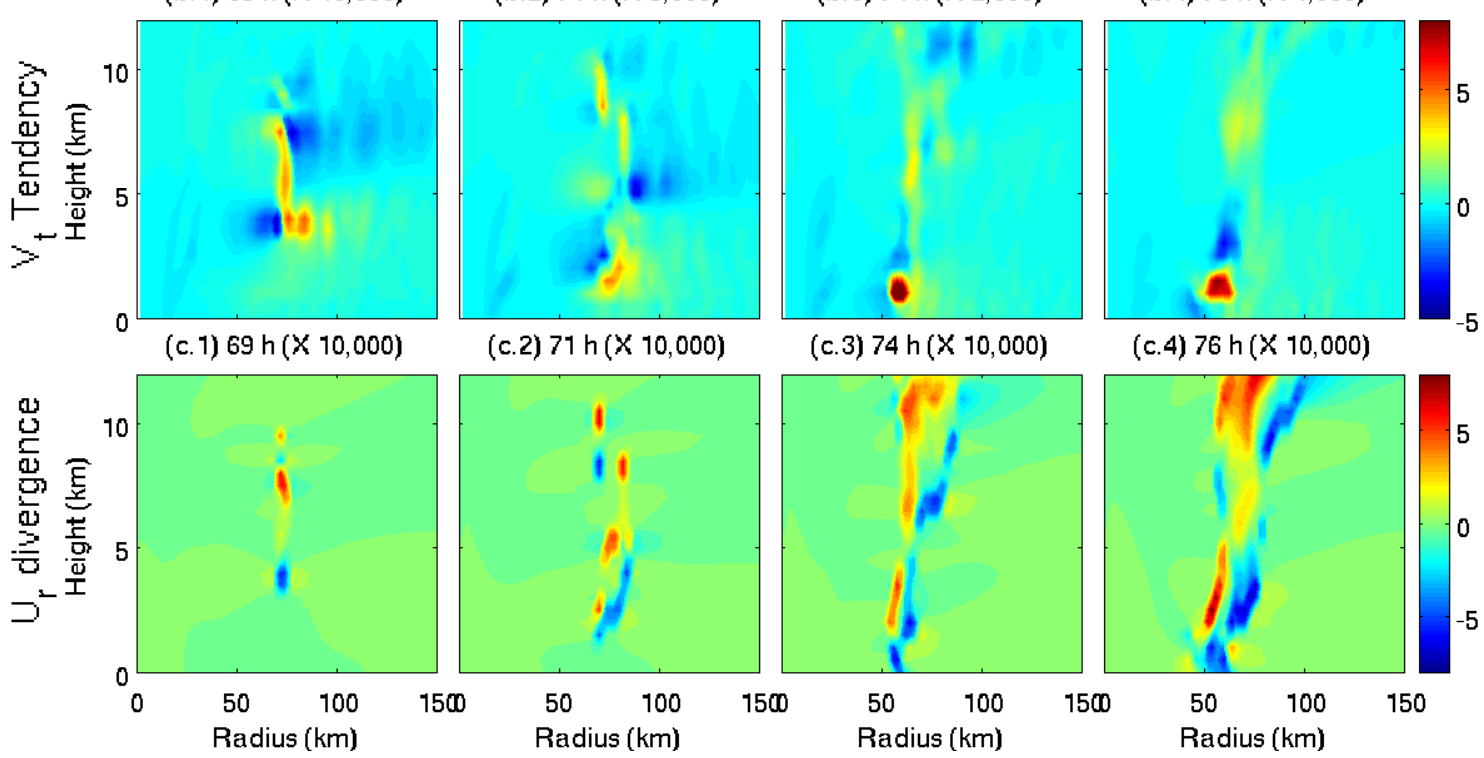

(c.4) $76 \mathrm{~h}(\times 10,000)$

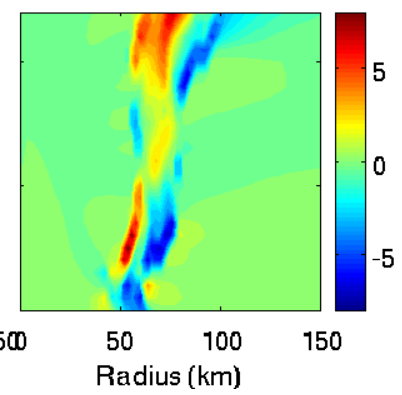

Figure 3.18 Outer rainband diabatic heating (upper row), tendency of tangential wind induced by the rainband heating (mid row), and divergence of radial flow induced by the rainband heating (bottom row) at different time. 
(a) Total RB heating $(\times 1,000)$

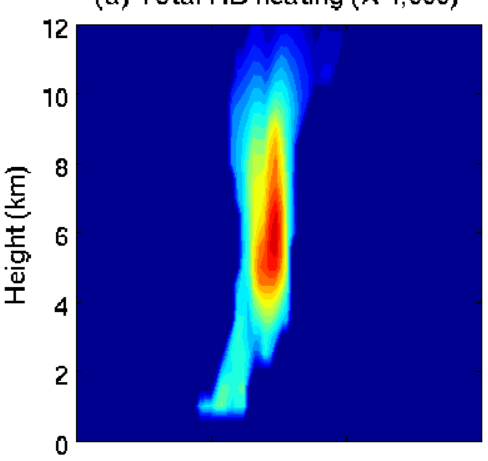

(b1) RB heating $>3 \mathrm{~km}(\times 1,000)$

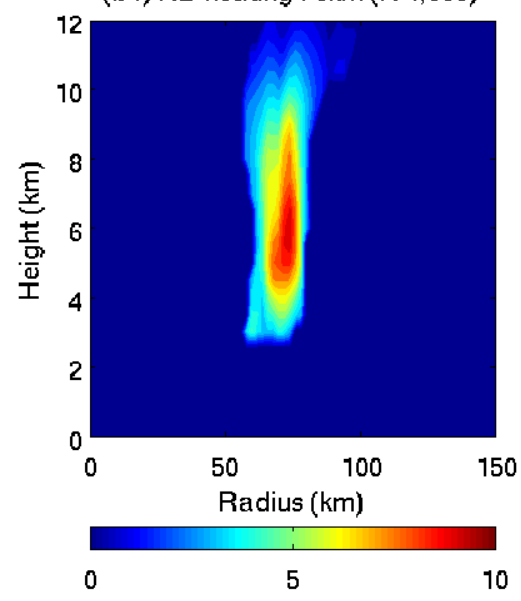

(a2) $V_{t}$ Tendency $(X 1,000)$

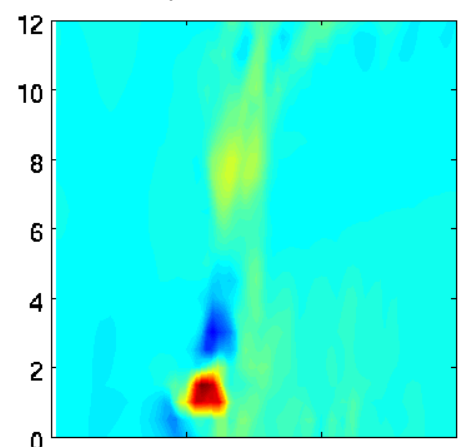

(b2) $V_{t}$ Tendency $(X 1,000)$

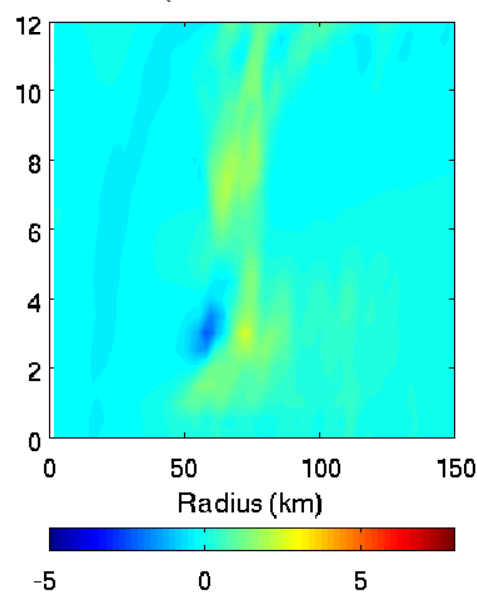

(a3) $U$ divergence $(X 10,000)$

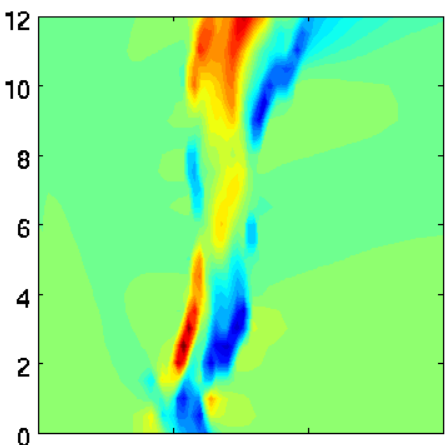

(b3) $U_{r}$ divergence $(\times 10,000)$

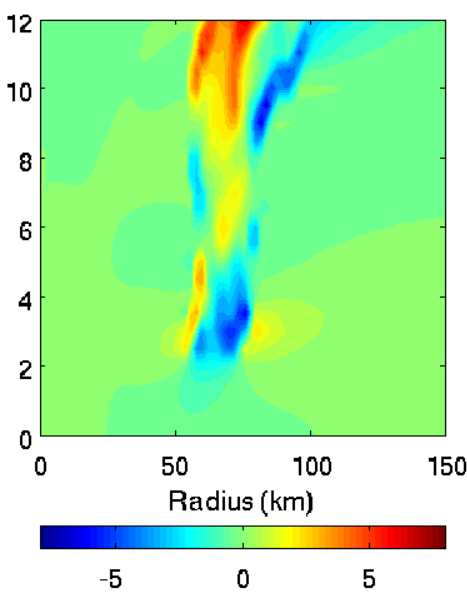

Figure 3.19 Upper row: Outer rainband diabatic heating and the induced tendency of tangential wind and divergence of radial flow at the $78^{\text {th }} \mathrm{h}$. Bottom row: The same as the upper row but with the heating below $3 \mathrm{~km}$ being removed. 
(a) Cloud water ( $g / \mathrm{kg}$ )

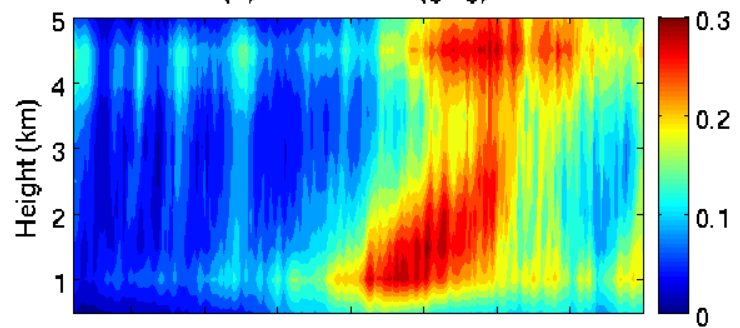

(c) Relative humidity (\%)

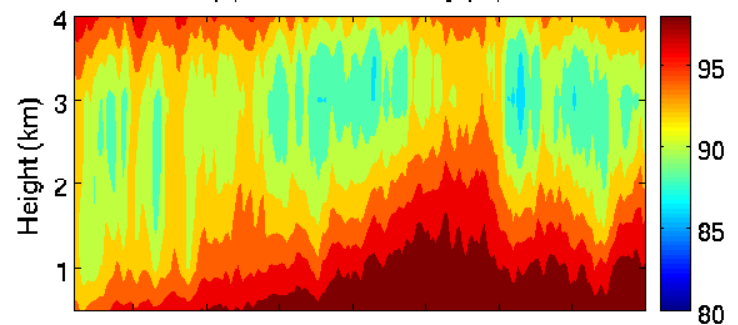

(e) $\Delta \theta=\theta(3 \mathrm{~km})-\theta(2 \mathrm{~km})(\mathrm{K})$

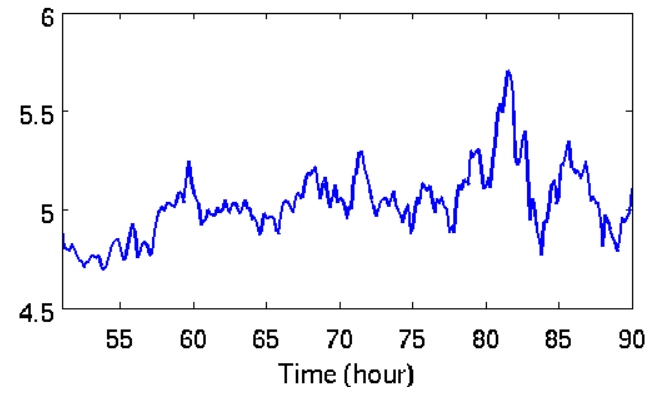

(b) Rain water ( $g / k g)$

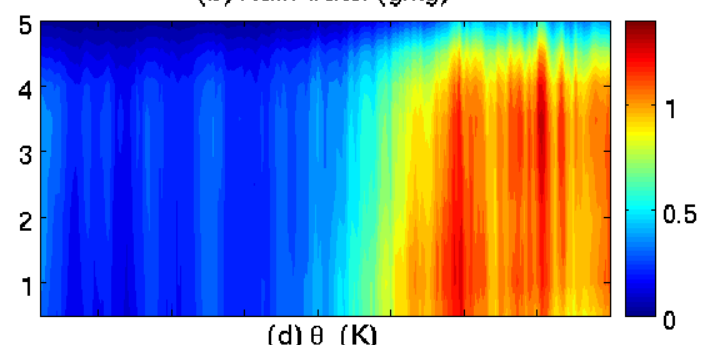

(d) $\theta_{\mathrm{e}}(\mathrm{K})$

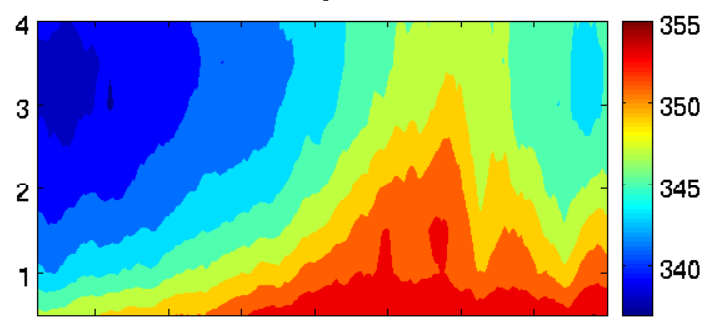

(f) Surface buoynacy flux (W/m2)

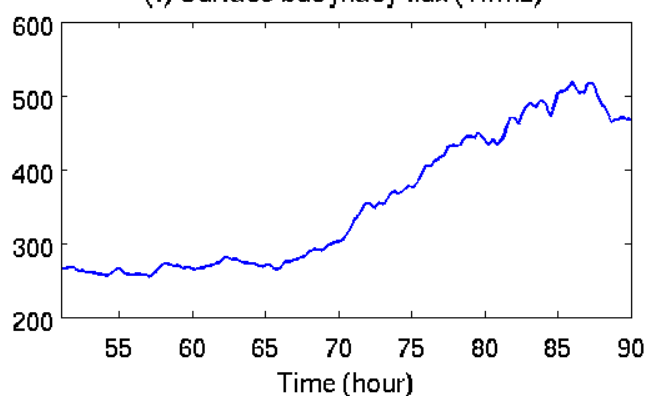

Figure 3.20 Time evolution of cloud water (a), rain water (b), relative humidity (c), equivalent potential temperature (d), difference of potential temperature between $3 \mathrm{~km}$ and $2 \mathrm{~km}$ (e), and surface buoyancy fluxes (f) averaged over the radii of $50-80 \mathrm{~km}$. 
(a) Time $=70 \mathrm{~h}$

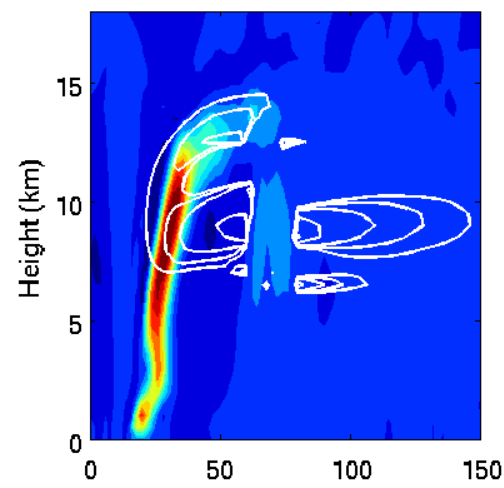

(d) Time $=76 \mathrm{~h}$

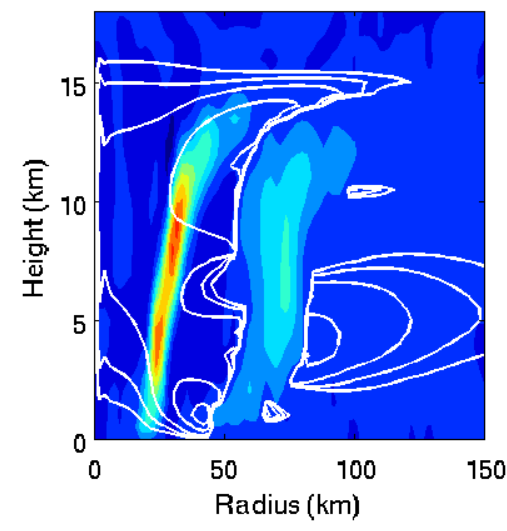

(b) Time $=72 \mathrm{~h}$

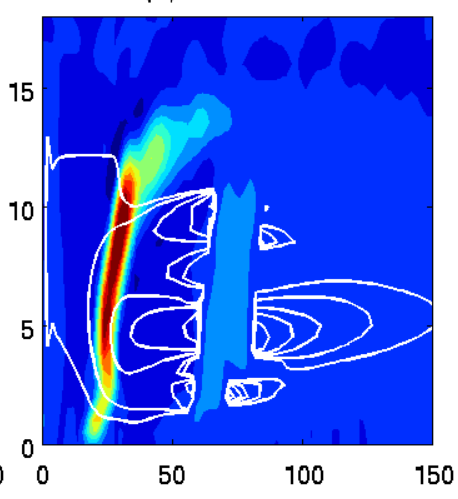

(e) Time $=78 \mathrm{~h}$

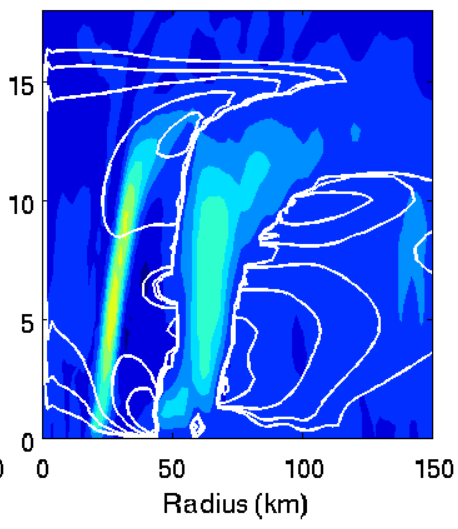

(c) Time $=74 \mathrm{~h}$

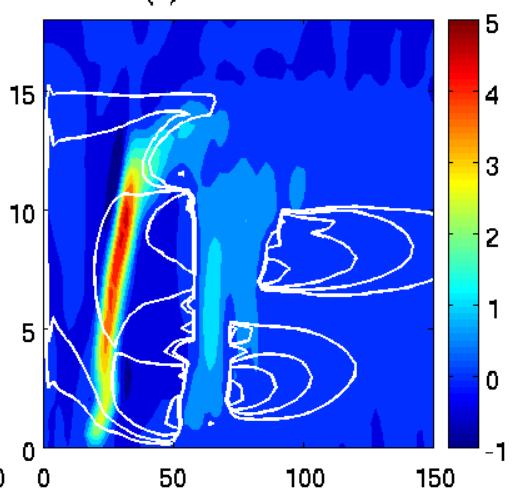

(f) Time $=80 \mathrm{~h}$

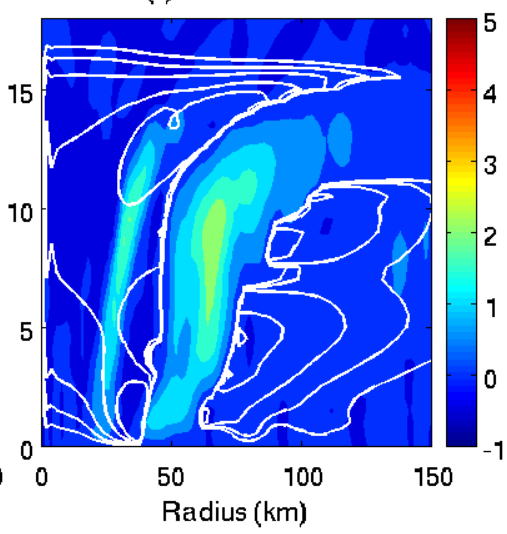

Figure 3.21 Subsidence (white contours) induced by the outer rainbad heating overlapped onto the vertical velocity (color shades) simulated by WRF. 
(a) Time $=70 \mathrm{~h}$

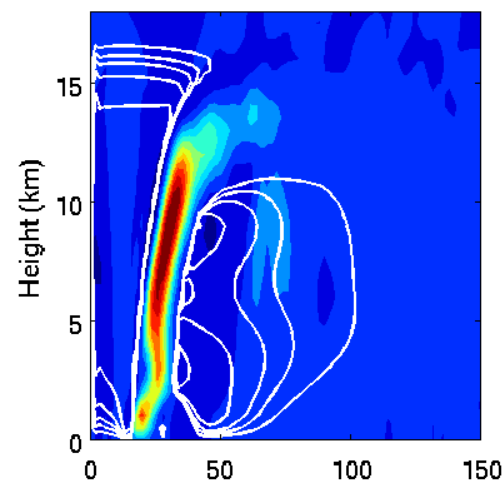

(d) Time $=76 \mathrm{~h}$

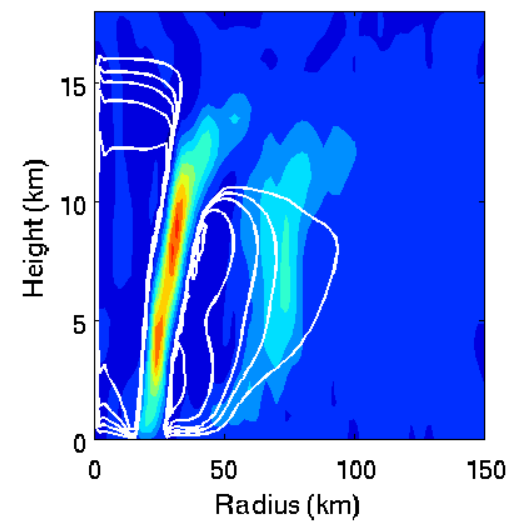

(b) Time $=72 \mathrm{~h}$

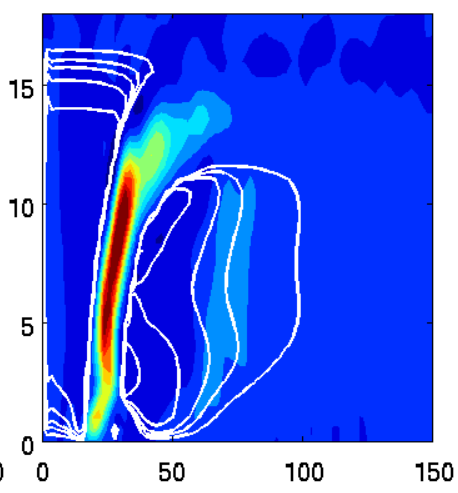

(e) Time $=78 \mathrm{~h}$

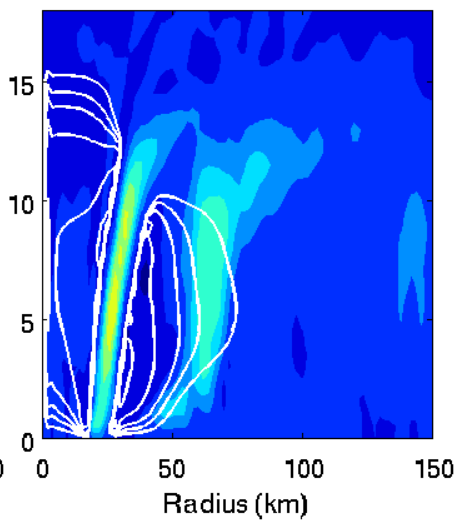

(c) Time $=74 \mathrm{~h}$

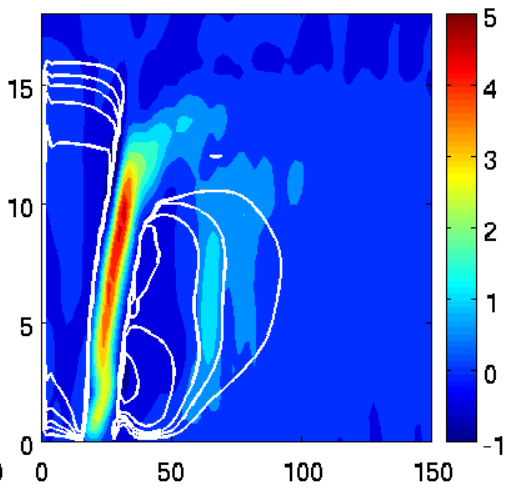

(f) Time $=80 \mathrm{~h}$

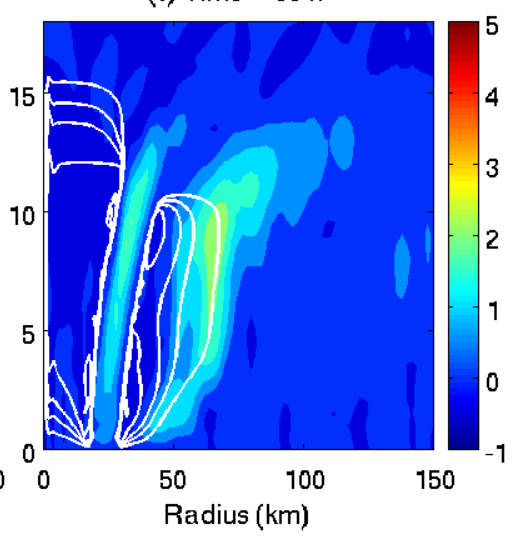

Figure 3.22 Subsidence (white contours) induced by the eyewall heating overlapped with the vertical velocity (color shades) simulated by WRF. 
(a) Time $=70 \mathrm{~h}$

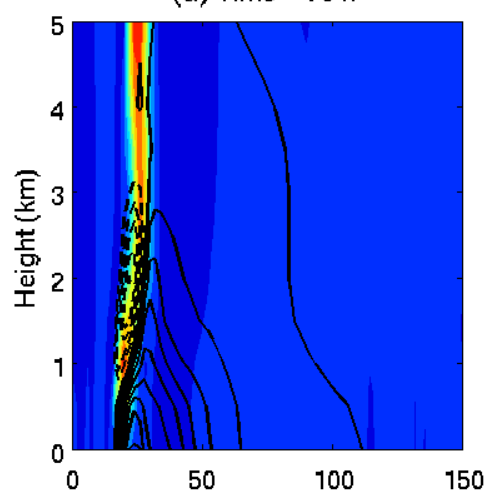

(d) Time $=76 \mathrm{~h}$

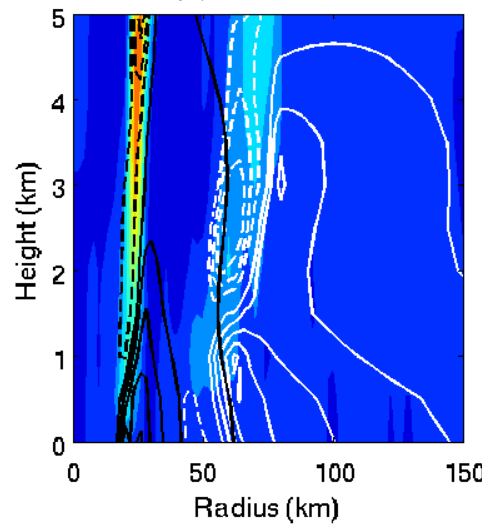

(b) Time $=72 \mathrm{~h}$

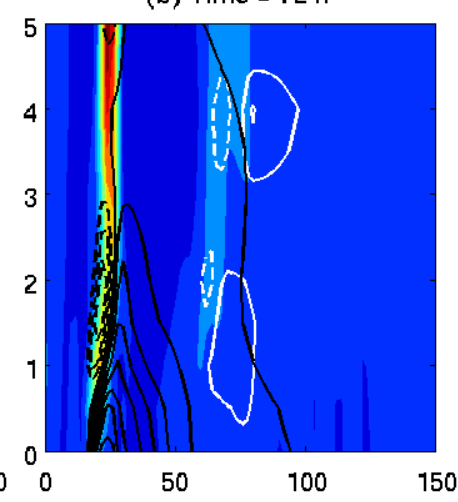

(e) Time $=78 \mathrm{~h}$

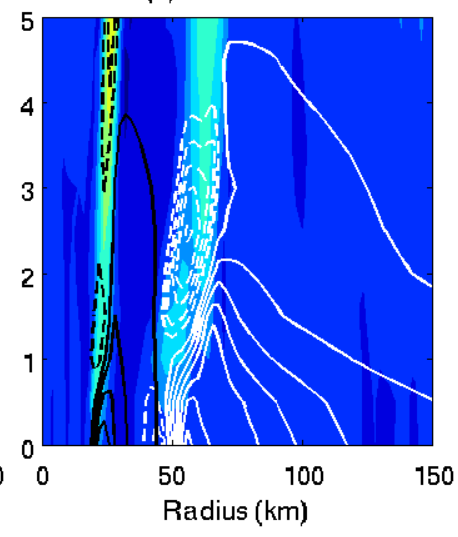

(c) Time $=74 \mathrm{~h}$

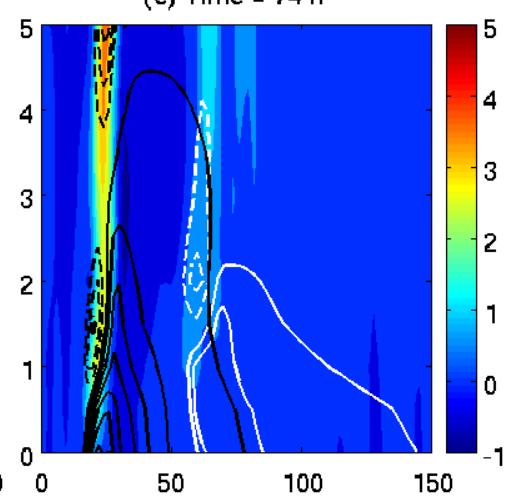

(f) Time $=80 \mathrm{~h}$

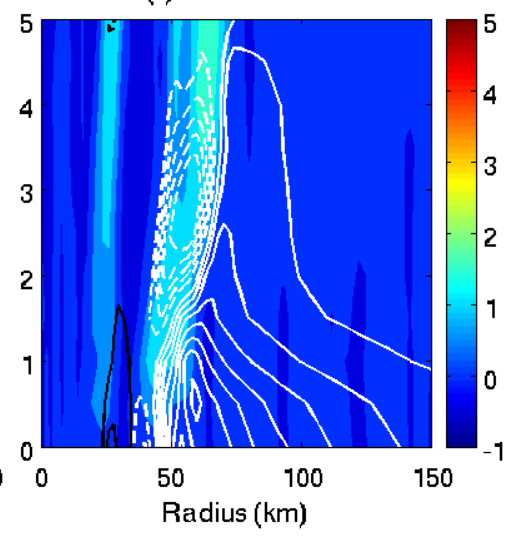

Figure 3.23 Radial flow induced by the eyewall heating (black contours) and outer rainband heating (white contours) overlapped onto the vertical velocity simulated by WRF. Radial inflow and outflow are indicated by solid and dashed contours, respectively. 
(a) NPS for wavenumber $>4$

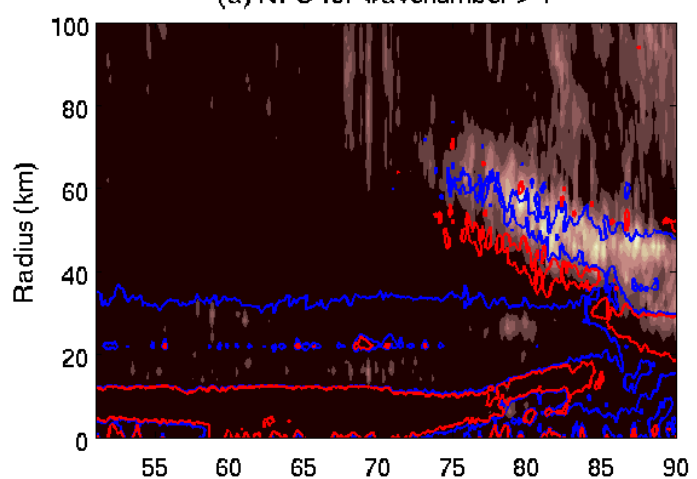

(c) NPS for wavenumber $=2$

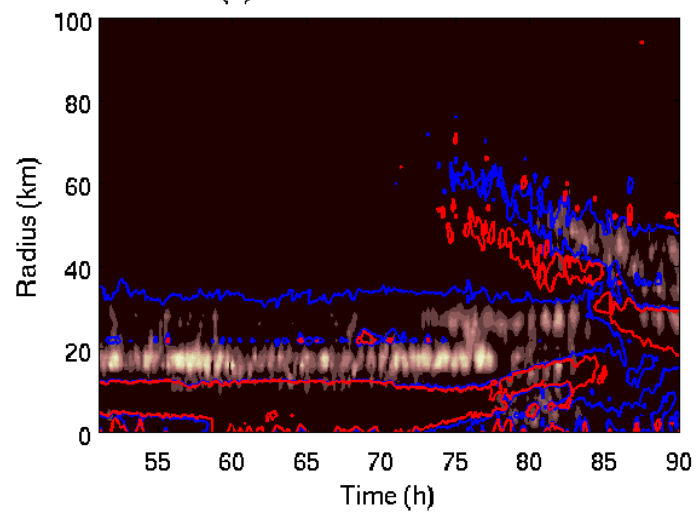

(b) NPS for wavenumber $=3$

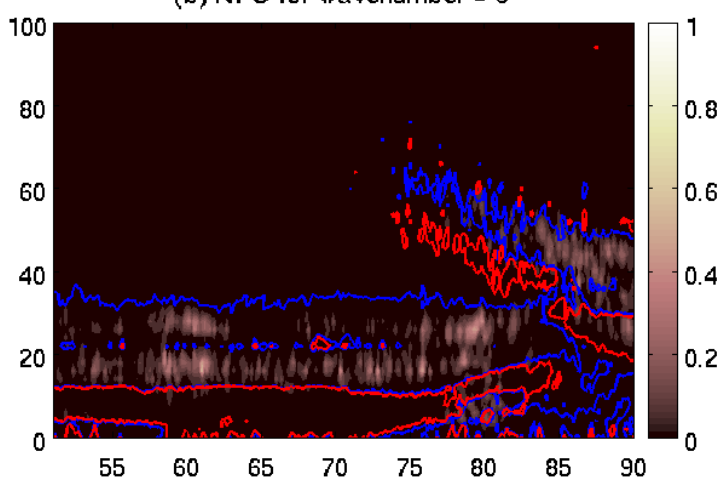

(d) NPS for wavenumber $=1$

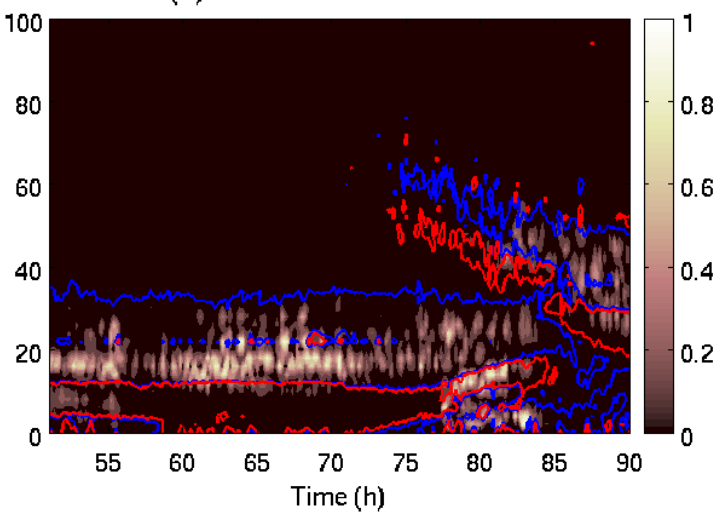

Figure 3.24 NPS of wavenumber 1, 2, 3, and sum of higher wavenumbers than 3 at the height of $2 \mathrm{~km}$ (shades) overlapped with the basic state PV radial gradient (contours). The blue and red contours indicate the PV radial gradient with value of $-5 \times 10^{-10}$ and $5 \times 10^{-10} \mathrm{~s}^{-1}$ respectively. 
(a) NPS for wavenumber $>4$

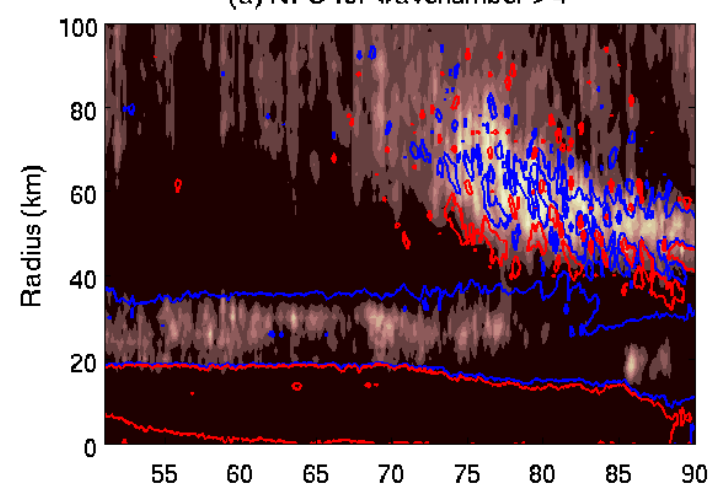

(c) NPS for wavenumber $=2$

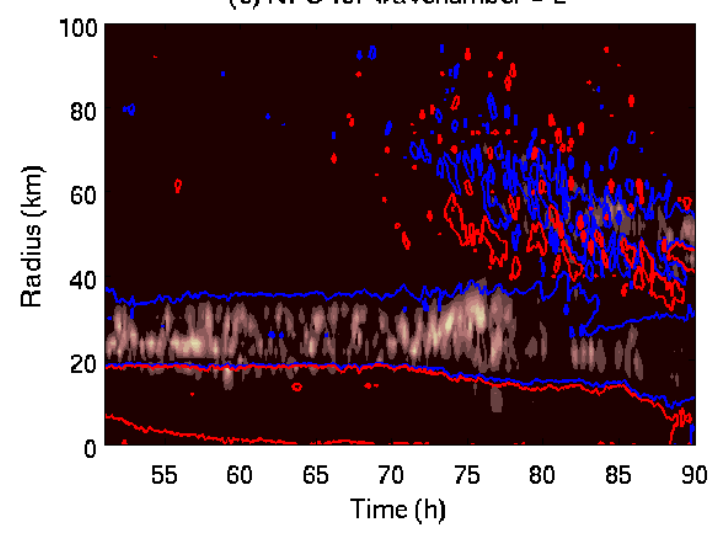

(b) NPS for wavenumber $=3$

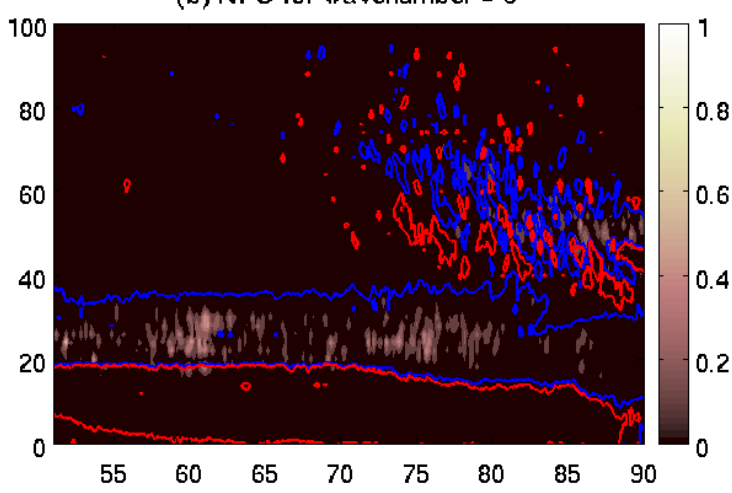

(d) NPS for wavenumber = 1

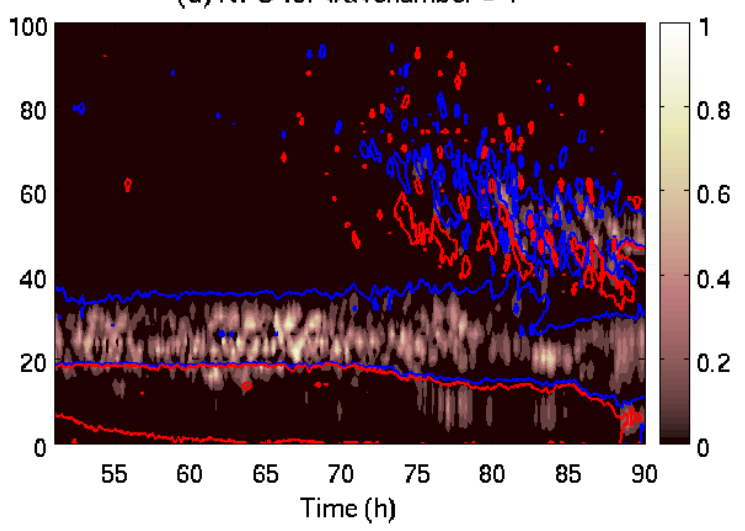

Figure 3.25 The same of Figure 3.24 but for NPS at $5 \mathrm{~km}$. 
(a) $72 \mathrm{~h}$

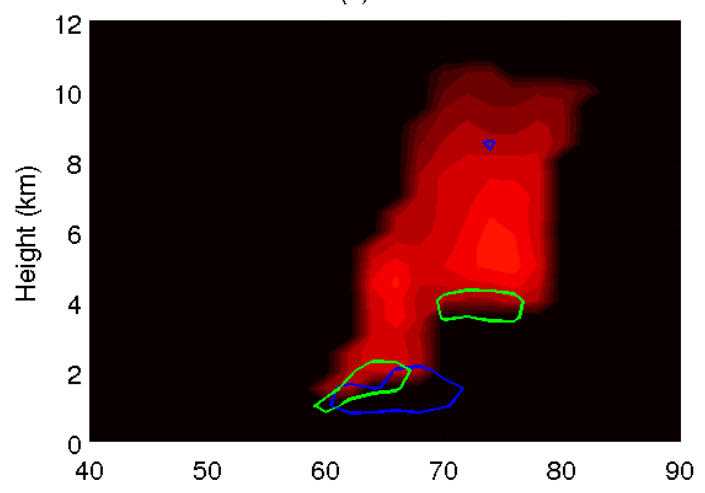

(c) $76 \mathrm{~h}$

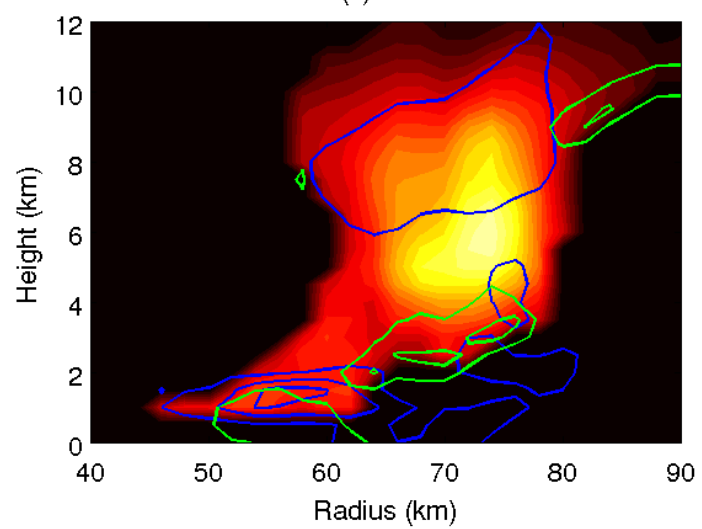

(b) $74 \mathrm{~h}$

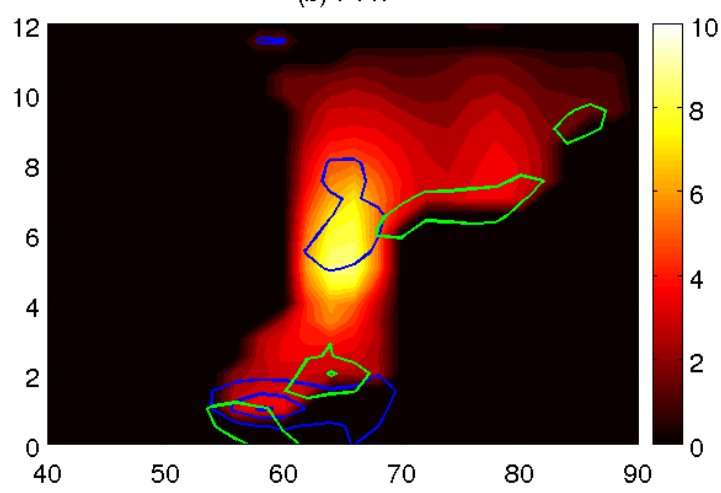

(d) $78 \mathrm{~h}$

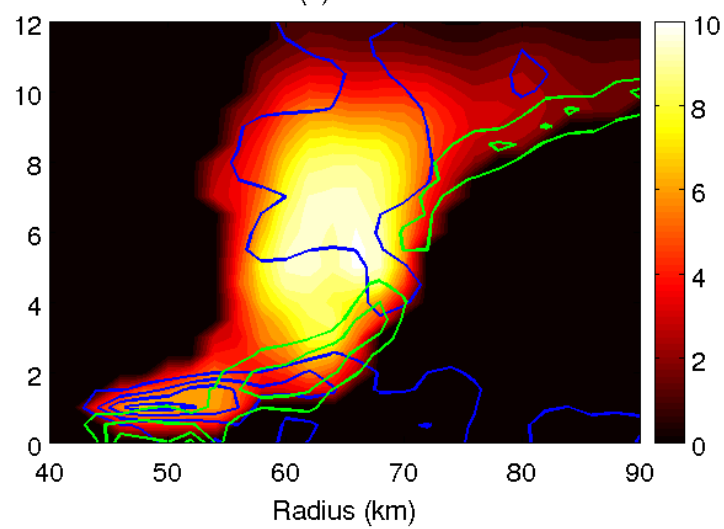

Figure 3.26 Radial-height plot of diabatic heating (shades) overlapped with convergence of radial flow (green contours) and symmetric contribution to the tendency of tangential wind at different time during ERC. The contours of radial flow convergence are: $-3 \times 10^{-4}$ and $-6 \times 10^{-4} \mathrm{~s}^{-1}$. The contours of tangential wind tendency are $1 \times 10^{-3}, 4 \times 10^{-3}$, and $7 \times 10^{-3} \mathrm{~ms}^{-2}$. 


\section{Chapter 4 Sensitivities of ERC to Model Physics}

As we demonstrated in Chapter 3, ERC in 3D numerical simulations results from a complicated interplay between eyewall and outer rainband convection involving both resolved and SGS processes. In particular, our analyses show that the tendency induced by the vertical SGS turbulent mixing is one of the leading contributions to the tangential wind budget. It is, thus, not a surprise that the occurrence, course, and characteristics of modeled ERCs depend on how the SGS processes are parameterized. In this chapter, we will present and discuss the sensitivities of ERC to vertical SGS turbulent mixing scheme, cloud microphysics, radiation, and structure of initial vortex.

\subsection{Sensitivity of ERC to vertical SGS turbulent mixing parameterization}

To investigate the sensitivity of ERC to SGS turbulent mixing parameterization, the exact same simulations as the control run are performed except that the MYJ scheme is replaced by the Yonsei University scheme (YSU, Hong et al. 2006) and the MellorYamada-Nakanishi-Nino-2.5 level TKE scheme (MYNN, Nakanishi and Nino 2004). These two sensitivity experiments are named as YSU run and MYNN run, respectively. Like the MYJ scheme, the MYNN scheme is a local turbulent mixing scheme based on the predicted TKE, but many parameters and coefficients used by the MYNN scheme to close the parameterization are different from those used by the MYJ scheme. Most importantly, the MYNN scheme is formulated with moist thermodynamics based on the variables conserved for moist reversible adiabatic processes, and thus, is often called a "moist" scheme in contrast to the MYJ scheme, which is often termed as a "dry" scheme. A recent study by Zhu et al. (2013) showed that the MYNN scheme tends to produce a 
much larger turbulent eddy exchange coefficients than the MYJ scheme in TC simulations and the difference in eddy exchange coefficients can have a profound impact on eyewall asymmetries and eyewall mesovortices. Below we will show that eddy exchange coefficients are also the important parameters that can affect SEF and ERC. The YSU scheme is a K-closure scheme but includes the effect of non-local mixing. It is also a "dry" scheme in the sense that it is not formulated with the variables conserved for moist reversible adiabatic processes. Both MYNN and YSU schemes use their own surface layer parameterizations, which are different in details although they are all formulated based on the Monin-Obukhov similarity theory. To be consistent with the control run, the Davis formula (Davis et al. 2008) is used to calculate the oceanic surface roughness in the MYNN and YSU experiments.

Like the control simulation, all sensitivity experiments are 8-day simulations, but our analyses here will focus only on the ERC period, the same as that in the control run that has been analyzed in Chapter 3. Figure 4.1 compares the vertical velocities at $5 \mathrm{~km}$ and tangential winds at $1 \mathrm{~km}$ from the YSU and MYNN experiments with those from the control run. The simulated vertical velocity and tangential wind fields show substantial differences due to the change in the vertical SGS turbulent mixing scheme. This is expected from many previous studies that showed that the structure and intensity of simulated TCs are sensitive to the boundary layer schemes (e.g., Li and Pu 2008; Nolan et al. 2009a, 2009b; Zhu et al. 2013). As indicated by the simulated vertical velocity and tangential wind fields, the control run with the MYJ scheme produces a much stronger TC than the YSU and MYNN schemes during this period. This is consistent with the numeri- 
cal simulations of a real hurricane Isabel (2003) by Zhu et al. (2013). Why the MYJ scheme tends to produce a stronger TC than other schemes is an interesting question but is beyond the scope of this study. We will investigate this issue in our future studies.

Figure 4.1 clearly shows that the simulated ERC goes through a different pass and has different characteristics. In the control simulation, there is a clean ERC associated with the development of a secondary maximum of tangential wind occurred at approximately $70-90 \mathrm{~h}$, which has been thoroughly analyzed in Chapter 3. In the YSU and MYNN experiments, the discontinuities in vertical velocity field seem to suggest that there are two consecutive ERCs with one right after the other occurring in a period approximately the same as that in the control run. However, the evolution of tangential wind in the YSU experiment shows that neither of the vertical velocity discontinuities is associated with the development of secondary maximum of tangential winds, suggesting that none of these two vertical velocity discontinuities may be considered as a real ERC since a secondary maximum of tangential wind is a key feature of an ERC. On the other hand, in the MYNN experiment, while the second vertical velocity discontinuity does not appear to involve the formation of a secondary maximum of tangential wind, the first one is accompanied by the development of a clean secondary maximum of tangential wind, and thus, like that in the control run, it may be considered as a real ERC. Looking closely at the vertical velocity fields, it suggests that there is a somewhat similarity between the control run and the two sensitivity experiments in that there is an outer rainband activity right after the ERC in the control run (Figure 4.1a), which may be considered to be analogous to the second outer rainband activity in the YSU and MYNN experiments. Howev- 
er, unlike the two sensitivity experiments, the secondary outer rainband activity does not generate a discontinuity in vertical velocity field. This is most likely due to the fact that the secondary outer rainband activity is too weak compared with the eyewall convection to lead to an ERC as we discussed before.

To better understand the evolution and structure of tangential wind, Figs. 4.2 and 4.3 show a series of height-radius plots of tangential wind during the ERC period from the YSU and MYNN experiments. In the YSU experiments, although there is an expansion of tangential wind (most obvious in Figure4.2d) similar to that in the control run that leads to the secondary wind maximum (Figure 3.7), there is no formation of secondary maximum of tangential wind throughout the simulation. Instead, we see a fairly steady slow increase of RMW with the expansion of the jet core of tangential winds. This confirms that the outer rainband activity that leads to the vertical velocity discontinuity does not generate a real ERC. The underlying mechanism for this phenomenon will be explored shortly in this section. In contrast to the YSU experiment, the height-radius tangential wind plots of the MYNN experiments clearly show that there is a clean secondary tangential wind maximum associated with the first vertical velocity discontinuity, sharing the similar characteristics to that of the ERC in the control simulation. However, like the YSU experiment, the second vertical velocity discontinuity does not lead to a secondary wind maximum but only an expansion of tangential wind, which results in a large core of tangential wind jet. To better understand the vertical and horizontal wind structure during the ERC period, Figs. 4.4 and 4.5 compare the instantaneous vertical velocity at the height of $5 \mathrm{~km}$ and horizontal wind speed at the height of $1 \mathrm{~km}$ during the two outer rain- 
band activities between the YSU and MYNN experiments. The vertical velocity fields clearly show that all four outer rainband activities lead to the concentric rings of convection. However, the horizontal wind speed shows that only the first outer rainband activity in the MYNN experiment generates a clean secondary wind maximum and the other outer rainband activities merely result in the expansion of the wind maximum associated with the inner eyewall. What are shown in Figs. 4.1- 4.5 suggests that the characteristics of ERC including the structure of TC vortex winds are sensitive to the SGS vertical turbulent mixing scheme. But before exploring the underlying mechanisms of the sensitivity, it is worthwhile to examine if the interaction between eyewall and outer rainband convection leading to the ERC in the control run discussed in detail in Chapter 3 is still the leading mechanism for the ERC observed in the MYNN experiment.

Figure 4.6, which is a similar plot to Figure3.9, shows the detailed tangential wind budget analyses averaged over $70-72 \mathrm{~h}$ from the MYNN experiment. It is clear that the main conclusions obtained from the control run still hold, which are worthy to be emphasized here again: (1) the net tendency of tangential wind is a small residual of a large cancellation between tendencies induced by the resolved and SGS processes (note that the net tendency in Figure 4.6a has been multiplied by a factor of 5); (2) The resolved processes are responsible for generating the positive tendency of tangential wind, and thus, are the driving force for SEF and ERC; (3) The horizontal advection of absolute vorticity and vertical advection of tangential wind are the two leading terms in the budget for generating positive tendency of tangential wind in the ERC region, although they have opposite signs within and above the boundary layer (Figs. 4.6d, 4.6e, and 4.6f); (4) 
The SGS vertical turbulent mixing is mainly responsible for generating negative tendency to cancel the positive tendency induced by the resolved processes; and finally, the resolved eddy processes and SGS horizontal turbulent mixing only play a minor role in SEF and ERC. These results suggest that the details in the SGS vertical turbulent mixing parameterization can substantially affect the occurrence and course of ERC although resolved processes are the driving force for ERC.

As demonstrated previously in Chapter 3, the resolved processes intimately involved in SEF and ERC are the outer rainband convective activities. Figure 4.7 shows the time evolution of azimuthal-mean diabatic heating, tangential wind, and net tendency of tangential wind during the ERC period from the MYNN experiment. It clearly shows that there are two outer rainband activities in the ERC period. The first one is approximately from the $61^{\text {st }} \mathrm{h}$ to $77^{\text {th }} \mathrm{h}$ when the outer rainband assumes the role of eyewall. During this period, there is a clean development of secondary maximum of tangential wind. Right after that, there is a second outer rainband activity which ends at the $93^{\text {rd }} \mathrm{h}$, during which the outer rainband evolves and eventually becomes the eyewall. However, although the outer rainband convection clearly generates a positive net tendency of tangential wind, no secondary maximum of tangential wind forms in the outer rainband region. There is only an expansion of tangential wind maximum associated with the primary eyewall. Comparing the two outer rainband activities, it is apparent that the secondary outer rainband moves inward at a greater speed and is able to approach much closer to the inner eyewall than the first one. Because of this, it leaves little room for the development of secondary 
wind maximum. The underlying mechanism of this difference will be explored in detail shortly.

Figure4.8 shows the Saywer-Eliassen equation diagnosed convergence of radial inflow and the acceleration of tangential wind induced by the horizontal advection of absolute vorticity and vertical advection of tangential wind at the height of $1 \mathrm{~km}$. Like what we showed in the control run, the convergence of radial inflow and the acceleration of tangential wind induced by the outer rainband convection go hand in hand, which is the main mechanism that leads to the SEF and ERC. The positive feedback among low-level convection, convergence of radial inflow, acceleration of tangential wind in the upper boundary layer, and surface evaporation that leads to the development of outer rainband convection and SEF in the control run still works for this case. This is supported by Figure 4.9, which shows the relative humidity and equivalent potential temperature at the height of $300 \mathrm{~m}$, surface buoyancy flux, and cloud water mixing ratio averaged over the radii from $50 \mathrm{~km}$ to $80 \mathrm{~km}$. The Sawyer-SEliassen diagnoses suggest that the convection in the outer rainband can result in convergence of radial inflow and acceleration of tangential wind in the boundary layer (Figure 4.8), which can enhance surface evaporation (Figure 4.9c) and lead to the effective transport of moisture into the convection to result in an increase of moisture in the low troposphere (Figs. 4.9a and 4.9b). The increased moist instability favors the further development of low-level convection. This can cause a runaway interaction leading to the development of outer rainband convection and subsequent SEF. The comparison between the control run and the MYNN experiment suggests that the fundamental mechanism that governs ERC remains the same. However, since 
ERC is a delicate process, in particular, the development of secondary wind maximum depends on a large cancellation between tendencies induced by resolved and SGS processes, the details of SGS parameterizations can substantially affect SEF and ERC.

One question that has not been answered here is why the second outer rainband activity in the MYNN experiment does not generate a secondary wind maximum just like the first one. The answer to this question may help us understand why ERCs do not occur in some numerical simulations (e.g., the YSU experiment) despite the vigorous outer rainband activity. To seek the answers to this question, we carefully analyzed the surface and moist convection processes during the first and second outer rainband activities. It turns out that much of the difference between the first and second outer rainband activity and the associated ERCs may be explained by the interaction between the surface processes and low-level moist convection. To clearly illustrate this mechanism, Figure 4.10 shows the radial distribution of diabatic heating, surface latent heat fluxes, 10-m wind speed, and friction velocity at an arbitrary time (here $65^{\text {th }} \mathrm{h}$ and $85^{\text {th }} \mathrm{h}$ are chosen) during the first and second ERC event respectively in the MYNN experiment. As the figure indicated, during the first ERC event there are two maxima of surface latent heat flux, 10-m wind speed, and friction velocity in the radial profile that correspond well with the eyewall and outer rainband heating and are cleanly separated by the moat. This radial structure is somewhat expected since convection can accelerate tangential wind and generate convergence of radial inflow in the upper boundary layer according to the SawyerEliassen diagnoses. The increased wind speed enhances turbulent intensity and promotes surface evaporation, which further favors the moist convection. In the meantime, the sub- 
sidence in the moat suppresses the convection, which leads to the weaker wind speed and smaller latent heat fluxes in the moat. On the other hand, the radial phase relationship between diabatic heating and surface variables in the second ERC event is quite different from that in the first event. What surprises us is that the maxima of surface latent heat flux, surface wind speed, momentum fluxes (measured by friction velocity) do not match with the heating, rather, the peaks reside somewhere in-between the eyewall and outer rainband heating. The shifting of the maximum surface wind radially from eyewall convection is consistent with vertical structure of tangential wind shown in Figure 4.7g. As the figure indicated, the core of tangential wind is shifted radially outward from the eyewall. This shifting results from both the acceleration of tangential wind by outer rainband convection and the de-acceleration associated with the eyewall. This radial phase shift in surface winds is very important for controlling the process of ERC. The strongest surface wind in the moat region (Figure 4.10f) enhances surface evaporation there to overcome the strong subsidence to result in the development of low-level convection in the moat, which is clearly seen from the diabatic heating (Figure $4.10 \mathrm{~b}$ ) at the $85^{\text {th }} \mathrm{h}$. This is the key difference between the first and second ERC events. In the first ERC event, the convection in the moat is really suppressed by the strong subsidence induced by the eyewall and outer rainband convection, which prevents the outer rainband convection from moving too close to the inner eyewall, and thus, leaves a sufficient room both in temporal and spatial to allow for the development of secondary maximum of tangential wind. In contrast, in the second ERC event the low-level convection fostered by the large surface evaporation due to the strong surface winds overcomes the moat subsidence, which serves as a mechanism to allow the outer rainband convection to approach much 
closer to the eyewall at a much faster speed, and indeed as indicated by Figure 4.7, the inward movement of outer rainband in the second ERC is faster than that in the first ERC. As a result, there is no sufficient room for the development of secondary maximum of tangential wind. To support this argument, Figure 4.11 shows the vertical velocity overlapped with the diabatic heating at the $65^{\text {th }} \mathrm{h}$ and $85^{\text {th }} \mathrm{h}$ from the MYNN experiment. In the first ERC event, the subsidence in the moat extends down at the surface, which separates the eyewall and outer rainband convection and prevents convection in the moat; whereas in the second ERC event the base of the subsidence is elevated to the midtroposphere $(>4 \mathrm{~km})$ due to the shallow convection developed in-between the eyewall and outer rainband deep convection, suggesting the important role of shallow convection generated by the boundary layer processes in governing the interaction between eyewall and outer rainband and the course of ERC.

To further diagnose the cause for the difference between the first and second ERC events, we analyzed the Sawyer-Eliassen diagnoses. Since the focus here is trying to understand the mechanism underlying the development of shallow convection in the moat, it is interesting to see how the eyewall and outer rainband heating contributes to this development. Like what we did before, to remove the noise, the eyewall and outer rainband heating is defined and separated using vertical velocity greater than $0.5 \mathrm{~m} / \mathrm{s}$. Figure 4.12 shows radial inflow and outflow induced by the eyewall and outer rainband diagnosed by the Sawyer-Eliassen equation. A key difference between the first and second ERC events is the radial inflow induced by the eyewall heating. In the first ERC event, the strong radial inflow (indicated by the contour of $-1 \mathrm{~m} / \mathrm{s}$ ) is really confined in the moat, which lim- 
its the moisture transported into the moat. In contrast, the radial inflow $(>1 \mathrm{~m} / \mathrm{s})$ induced by the eyewall heating in the second ERC event is much stronger than that in the first event and extends far beyond the outer rainband. Apparently, the moisture transported into the moat by strong radial inflow induced by the eyewall heating provides an important energy source for the development of shallow convection in the moat. Comparing the eyewall diabatic heating at the two different times, it suggests that the vertical structure of the heating is the likely cause for the different radial inflow. In the first ERC event, the strongest eyewall heating is located above freezing level at approximately $6 \mathrm{~km}$; whereas the center of the strongest eyewall heating in the second ERC event is at the lower altitude of $5 \mathrm{~km}$ approximately at the freezing level. To confirm this, we did a Sawyer Eliassen experiment to artificially place the center of the eyewall heating at the $65^{\text {th }} \mathrm{h}$ at lower altitudes, the results show that the heating at lower altitudes generates stronger radial inflow in the boundary layer and extends farther radially outward. Since convection below and above the freezing level involves different cloud microphysics, this result suggests that ERCs are sensitive to the representation of cloud microphysics in numerical model. This issue will be discussed in the next section.

As shown previously in Figs. 4.1d, 4.2, 4.5c, 4.5d, there are two ERC events in the YSU, but none of them is able to produce secondary maximum of tangential wind, a common feature associated with observed ERCs in real TCs. The question here is: is the reason for failure to produce secondary maximum of tangential wind in the YSU experiment the same as that in the second ERC event in the MYNN experiment? To answer this question, we did the similar analyses by looking at the radial phase relationship between 
diabatic heating and surface processes. Figure 4.13 shows the radial distribution of diabatic heating, surface latent heat flux, 10-m wind speed, and friction velocity at an arbitrary time in the two ERC events of the YSU experiment. The radial phase relationship between the diabatic heating and surface evaporation in the YSU experiment exhibits the same characteristics as that in the second ERC event of the MYNN experiment, suggesting that the mechanism of controlling the shallow convection in the moat that we found previously in the MYNN experiment still holds in the YSU experiment. This is interesting considering that the MYNN and YSU schemes are formulated based on different concepts as summarized previously. This result implies that the impact of vertical turbulent mixing parameterization of ERC can be understood despite the fact that schemes are different in many aspects.

However, the fact that the MYNN scheme does produce an ERC with a clean secondary wind maximum suggests that there must be something different between the two schemes in terms of their impact on ERCs. As we showed previously one process that can substantially affect the course of an ERC is the shallow convection in the moat, thus, our focus is on understanding the physical controls of the development of shallow convection. In addition to the surface evaporation, which is an important source of energy for convection, the other important parameter that is critical to convection is the atmospheric stability. Figure 4.14 compares the radial distribution of $2-\mathrm{m}$ potential temperature with respect to diabatic heating during the ERC events between the MYNN and YSU experiments. In the MYNN experiment, the 2-m potential temperature is the highest at the storm center, and it gradually decreases radially outward. This structure appears to be consistent with 
the observed radial temperature profile in Hurricane Frances (2004) by the NOAA WP3D aircraft (Rozoff et al. 2008). However, we note that the aircraft observed profile is obtained from a particular flight leg whereas the simulated profile is an azimuthal mean. Also, it is important to keep in mind that the flight level temperature profile can be substantially different from the 2-m temperature. The examination also shows that the surface temperature profile in the MYNN experiment shares the similar characteristics to that in the control run (not shown here). Interestingly, the radial profile of 2-m potential temperature in the YSU experiment has different characteristics from that in the MYNN experiment and the control run. Unlike the radial temperature profile in the MYNN experiment, which gradually slopes radially outward, the coldest temperature occurs in the moat in-between the eyewall and outer rainband in the YSU experiment. This radial temperature structure is very important for the shallow convection in the moat. Since the SST $\left(29^{\circ} \mathrm{C}\right)$ stays the same in all experiments, the lowest 2-m temperature in moat in the YSU experiment indicates that the surface air is most thermally unstable in the moat, which favors the development of shallow convection there. Because of this and plus the large surface latent heat fluxes, the vigorous shallow convection in the moat causes the outer rainband convection to be able to move quickly inward and approach and merge with the inner eyewall. As we argued before, this leaves little room both temporally and spatially to develop a secondary maximum of tangential wind. It is not clear, however, why the YSU scheme produces different temperature radial profile in the surface layer from that in the MYNN experiment and control run. A possible reason is that the YSU uses its own surface layer parameterization, which is different from that used with the MYNN and MYJ schemes. To clarify this issue, further investigation is needed. 
The vertical turbulent mixing schemes examined in this study were developed independently and differ in many aspects including the associated surface layer parameterization, and thus, it is not easy to understand what exact components in the schemes lead to the different characteristics of ERCs. To isolate the problem, we performed an extra experiment with the MYJ scheme in which everything else is the same except that we doubled the mixing length in the boundary layer during the simulation (the experiment is named as MYJ-BL2 hereafter). The design of this experiment is based on the consideration that turbulent eddy viscosity can have a substantial impact on the TC inner core structure according to recent studies. The change in mixing length, and thus, the change in eddy viscosity allows us to isolate and examine the effect of eddy viscosity on ERC. Figure 4.15 compares the vertical velocity at $5 \mathrm{~km}$ and tangential wind at $1 \mathrm{~km}$ between the control run and the MYJ-BL2 experiment. The time evolution of vertical velocity field in the MYJ-BL2 experiment exhibits two discontinuities associated with the outer rainband activities similar to those in the MYNN and YSU experiment, suggesting that there are two ERCs occurred in this period. It appears that the second outer rainband activity suppressed in the control run is fully developed due to the increase of mixing length. However, as the tangential wind field indicated, the clean secondary maximum of tangential wind associated with the first outer rainband activity in the control run is no longer clearly seen in the MYJ-BL2 experiment. This is confirmed by Figure 4.16, which shows the time evolution of radius-height structure of tangential wind during the ERC period. Like what we showed before, there is an expansion of tangential wind resulting from the outer rainband activity during each ERC event, but no secondary maximum of tangential wind occurs at the top of the boundary layer, rather, we see a double-peak structure 
roughly above $2.5 \mathrm{~km}$ and $4 \mathrm{~km}$ at the $81^{\text {st }}$ and $93^{\text {rd }} \mathrm{h}$, respectively, suggesting that there is an elevated concentric ring structure of tangential wind in this case. Similar phenomenon is also seen in the YSU experiment (e.g., $\sim 73^{\text {rd }}$ h) and MYNN experiment (e.g., $\sim 89^{\text {th }} \mathrm{h}$ ). Such elevated concentric rings of tangential wind may be explained by the vertically tilted structure of outer rainband convection. As shown in Figs. 4.17a and 4.17b, the outer rainband convection tilts toward the eyewall with the decrease of height. Since convection can accelerate tangential wind according to the Sawyer-Eliassen analyses, the distant eyewall and outer rainband convection aloft tends to generate concentric rings of tangential wind, but the nearly merged eyewall and outer rainband convection at low levels can hardly generate separate peaks of tangential wind. As we argued previously, the reason that the outer rainband convection is able to move closer to the eyewall in the low altitudes is due to the shallow convection developed in the moat fostered by the large surface heat fluxes there. This mechanism still holds in this case as supported by Figure 4.17, which shows that the strongest surface wind and largest surface latent heat flux reside in the moat in-between the eyewall and outer rainband, a feature similar to that in the second ERC event in the MYNN experiment and the two ERC events in the YSU experiment.

Since the only difference between the control run and MYJ-BL2 experiment is the mixing length, the similar characteristics of ERC among the MYNN, YSU, and MYJBL2 experiments shown previously suggest that the vertical eddy viscosity is an important parameter that can substantially affect the characteristics and course of an ERC. Such an impact on ERC is mainly through the modulation of shallow convection in the 
moat by the vertical turbulent mixing processes. As we showed here using the SawyerEliassen analyses, the low-level convection has an ability to accelerate tangential wind and induce convergence of radial inflow in the boundary layer, which can enhance surface evaporation to further promote convection via a positive feedback. Because of this, the shallow convection in the moat generated by the boundary layer turbulent mixing in this case serves as a mechanism to allow outer rainband convection to move toward the eyewall in the low levels. This appears to be a key process to determine the duration of an ERC and the tangential wind structure (i.e., whether or not to have a secondary maximum at the top of the boundary layer or an elevated concentric rings) of the ERC. However, it should be emphasized that the vertical eddy viscosity is not the only parameter that can affect ERC. As indicated by Figs. $4.17 \mathrm{~g}$ and $4.17 \mathrm{~h}$, the change of mixing length does not appear to have an impact on determining the radial profile of surface air potential temperature, which also plays a role in controlling the low level convection by altering the atmospheric stability. As we argued before, such change in surface air temperature radial profile is likely caused by the surface layer parameterization. But to clarify this issue, further investigation is needed.

\subsection{Complications of ERC in numerical simulations}

The focuses of our study so far are on demonstrating the role of outer rainband convection in governing ERC and how SGS vertical turbulent mixing affects the concentric ring structure of tangential wind associated with ERC via the interaction between surface processes and shallow convection in the moat. In this section, the robustness of our previous findings and further complications of ERC in numerical simulations will be ex- 
plored. We will briefly present the sensitivity of simulated ERC to initial structure of vortex, other model parameterizations, and background wind, and raise scientific questions for future study.

\subsubsection{Sensitivity of ERC to initial structure of vortex}

All simulations analyzed previously are initialized from the same idealized vortex, thus, it is unclear if our findings are universal or pertain only to this particular setting of vortex. To answer this question, three additional experiments are performed to investigate the sensitivity of simulated ERCs to the change in RMW, PV skirt, and circular structure of the initial vortex. Figure 4.18 compares the radial profiles of vorticity and tangential wind of the initial vortex between the sensitivity experiments and control run. In the first experiment, the RMW of the initial vortex configured in the control run is doubled. This is to examine how the size of a vortex might affect ERC. In the second experiment, the solid vortex in the control run is adjusted to an annulus ring of vortex. This change is based on the consideration that the solid and annulus vortex have different dynamic instabilities to PV perturbations according to the VRW theory (Schubert et al. 1999). In the third experiment, the initial vortex is adjusted to have a large PV skirt. According to Montgomery and Kallenback (1997), the outward propagation of VRW requires negative radial PV gradient, thus, a large PV skirt will support the outward propagation of eyewall disturbances. This will allow us to examine if outward propagation of VRWs generated by the inner eyewall can affect ERCs in our simulations. Figure 4.19 compares the vertical velocity at $5 \mathrm{~km}$ and tangential wind at $1 \mathrm{~km}$ between the control run and sensitivity experiments. Like the control run, all sensitivity experiments generate a clean ERC with 
concentric tangential wind maxima corresponding to the inner and outer eyewall. The time evolution of vertical velocity clearly indicates that all these ERC events are initiated from the outer rainband convection. The detailed procedure of how sporadic outer rainband convection evolves into a concentric outer ring of convection has been thoroughly analyzed in Chapter 3. The same Sawyer-Eliassen analyses are applied to all these sensitivity experiments. Similar results to those of the control run are obtained, suggesting that the major finding of this study still holds regardless of the structure of initial vortices. However, the simulated ERCs in these experiments do show substantial differences. Both large PV skirt and doubling RMW cause the ERC to occur at an earlier time and yield a much wider moat between the inner and outer eyewall than that in the control run. Among them, the moat in the large PV skirt experiment possesses the widest radial width. It appears that the width of moat, to a large extent, determines the structure and duration of concentric rings of tangential wind, which can be seen more clearly by comparing the height-radius structure of tangential winds from different experiments. Figs. 4.20 and 4.21 show the time evolution of azimuthal-mean tangential wind in the doubling RMW and large PV skirt experiments. The secondary maximum of tangential wind in the doubling RMW experiment approximately forms at the $63^{\text {rd }} \mathrm{h}$ and ends at $68^{\text {th }} \mathrm{h}$. The duration of concentric rings of tangential wind ( $\sim 5$ hours) in this experiment does not appear to be substantially lengthened compared with that in the control run (Figure 3.7) despite a wider moat. This is quite different from the large PV skirt experiment in which the secondary tangential wind maximum starts at about $47^{\text {th }} \mathrm{h}$ and lasts over 18 hours till $65^{\text {th }} \mathrm{h}$. The duration of concentric rings of tangential wind is substantially lengthened due to the further increased radial width of moat. This result suggests that a critical width of moat 
might exist beyond which the acceleration of tangential wind induced by the outer rainband convection can be cleanly separated from that induced by the inner eyewall convection. It is also true reversely that it might not be able to generate a secondary maximum of tangential wind if the moat is too narrow as the case of the ERC in the YSU experiment. It is not clear, however, why larger PV skirt tends to produce a wider moat. To understand the underlying mechanism, further investigation is needed, but it suggests that different characteristics of ERC shown in observations and previous numerical simulations could be caused by different structures of TC vortices.

In the annulus experiment, again we see double-eyewall replacement event during the ERC period similar to what we've seen in the MYNN, YSU, and MYJ-BL2 experiments. The detailed examination shows that like the ERCs in the MYNN and MYJ-BL2 experiments only the first ERC event generates a secondary maximum of tangential wind and there is no concentric ring structure of tangential wind in the second ERC event. This result suggests that the double-eyewall replacement is not a unique phenomenon pertaining only to certain SGS vertical turbulent mixing schemes. It can be generated due to the change in vortex structure. Since double-eyewall replacement does not occur in the large PV skirt and doubling RMW experiments, the annulus ring of PV, small PV skirt, and small RMW are likely the favorable conditions for generating this phenomenon. However, the exact reason for causing the double -eyewall replacement is currently not clear. It should be noted here that all simulations performed in this study are idealized simulations starting from an artificially constructed TC-like vortex. Thus, observational studies are required to assess whether-double eyewall replacement is realistic and numerical simula- 
tions with real TCs are needed to further explore the underlying mechanisms of this phenomenon.

\subsubsection{Sensitivity of ERC to snow terminal velocity}

In the previous section, we have shown that diabatic heating from the eyewall and outer rainband convection play an important role in governing ERC. In numerical simulations, the magnitude and distribution of diabatic heating largely depend on cloud microphysics. Thus, it is interesting to see how the changes in microphysics might affect ERCs. A microphysical scheme consists of many complicated components. It is not realistic to look into all of them in a dissertation study. Here we only examined the sensitivity of ERC to snow terminal velocity, a parameter that determines the falling of snow. We performed two sensitivity experiments by doubling the snow terminal velocity and reducing the snow terminal velocity by half. Figure 4.22 compares the vertical velocity at $5 \mathrm{~km}$ and tangential wind at $1 \mathrm{~km}$ between the control run and sensitivity experiments. It shows that doubled snow terminal velocity causes the outer concentric eyewall to move much closer to the inner eyewall. Because of this, it leaves little room for the development of a secondary maximum of tangential wind as we discussed previously. As a result, only an expansion but no concentric ring structure is seen in the tangential wind field. On the other hand, the reduced snow terminal velocity increases the moat width of the first ERC event, which leads to the development of a much clean secondary maximum of tangential wind with a longer duration. In addition, the reduced snow terminal velocity also enhances the second outer rainband activity to allow it to develop into the second ERC event, similar to what we have seen in other sensitivity experiments. As we argued previously, 
the double-eyewall replacement phenomenon may be related to the specific setting of the initial vortex used in this study.

To understand the impact of snow terminal velocity on ERC shown in Figure 4.22, these three simulations are further analyzed and compared. Again, we attempt to address this issue within the same symmetric framework as we used in the previous analyses from the perspective that ERC is controlled by the interaction between eyewall and outer rainband convection. Our argument on this issue may be better understood with the assistance of Figure 4.23, which shows the diabatic heating and the radial inflow in the boundary layer at an arbitrary time during the ERC period. As the figure indicated, large snow terminal velocity enhances the diabatic heating. This is expected since the large terminal velocity causes the snow to fall quickly and melt in the lower level, which enhances heating in the upper level. In contrast, small terminal velocity makes it difficult for snow to fall, and thereby reduces the diabatic heating. As a response, the strong diabatic heating tends to induce a strong radial inflow in the boundary layer, and indeed, the enhanced diabatic heating induced by the inner eyewall in the doubling snow terminal velocity experiment results in a much stronger radial inflow than that in the reduced snow terminal velocity experiment. Even in the moat area the radial inflow with a value greater than 12 $\mathrm{m} / \mathrm{s}$ can have a depth comparable to that outside the outer rainband. As we argued before, the moist air with a large enthalpy transported into the moat is one of the likely mechanisms to overcome the strong subsidence induced by eyewall and outer rainband convection and promote shallow convection there that allows the outer rainband convection to move closer to the inner eyewall. On the other hand, the weak inner eyewall heating is 
unable to generate a radial inflow sufficiently strong to foster shallow convection in the moat, and thus, causes a clean separation between the inner and outer eyewalls and leads to the concentric rings of tangential winds. However, the actual process due to the change in snow terminal velocity may be much more complicated than what we described here. We note that the anticipated relationship between the change in diabatic heating and the change in snow terminal velocity appears only valid in the inner eyewall. As shown in Figure 4.23 , the diabatic heating of the outer rainband in the reduced snow terminal velocity experiment is not the weakest among the three simulations. It is still unclear why it does not generate the weaker diabatic heating in the outer rainband than that in the control run as expected. Further analyses are needed to clarify this issue.

\subsubsection{Sensitivity of ERC to radiation}

In all simulations presented previously, although the simulated ERCs show substantially different characteristics due to the changes in SGS vertical turbulent mixing scheme and microphysics, all ERCs occur at approximately the same time. Even for different conditions of the initial vortex, for example, the large PV skirt and doubling RMW, the simulated ERCs occur only slightly earlier (less than 20 hours, Figure 4.19). From the numerical forecast perspective, this result is encouraging since the timing of ERC does not strongly depend on these factors. If SGS vertical turbulent mixing, cloud microphysics, or even the change in vortex structure in a certain range cannot substantially affect the timing of ERC, then, what else is critical to the timing of ERC? It turns out that radiation is one of the key processes that may impose a strong impact on the timing of ERC. We performed an extra experiment, which is exactly the same as the control run but with 
longwave and shortwave radiation turned off. Figure 4.24 compares the vertical velocity at $5 \mathrm{~km}$ and tangential wind at $1 \mathrm{~km}$ in the no-radiation experiment with those from the control run. It shows that the outer rainband activity in the no-radiation experiment is exceptionally quiet in almost entire simulation till the very end of the simulation when the development of outer rainband convection leads to an ERC event. The question is why turning off radiation suppresses outer rainband convection, and thus, substantially delays ERC. This is an interesting question and a complete understanding may require an indepth analysis. One possibility is that the convective instability of the lower troposphere is substantially different with and without cloud top radiative cooling. Although cloudtop radiative cooling may not be critical to deep convection, which is fundamentally buoyancy driven, it is the driving forcing for turbulent mixing in shallow convection. A key role of shallow convection is to enhance vertical transport of energy and moisture in the low troposphere and moisten mid-troposphere, which provides a favorable condition to promote deep convection. Therefore, the cause for the exceptionally weak outer rainband activity in the no-radiation experiment is most likely due to the lack of cloud-top radiative cooling by turning off radiation. This result suggests that the radiative transfer calculation could be critical for a timely numerical forecasting of ERC.

\subsubsection{Impact of background wind on ERC}

The findings of physical controls of ERC and sensitivity of ERC to model physics and initial condition are obtained from the TC-like vortices in the quiescent condition. In the real world, TCs are commonly embedded in an environment with background wind. In order to show that the background wind might add complications to ERC, two addi- 
tional sensitivity experiments with prescribed background wind of $5 \mathrm{~m} / \mathrm{s}$ and $10 \mathrm{~m} / \mathrm{s}$ are performed. Figure 4.25 shows the vertical velocity at $5 \mathrm{~km}$ and tangential wind at $1 \mathrm{~km}$ from these two experiments compared with those from the control run. In the experiment with $5 \mathrm{~m} / \mathrm{s}$ background wind, the ERC is no longer as clear as that in the control run although the discontinuities of vertical velocity associated with the ERC can still be identified, but no concentric ring of tangential wind is seen. When the background wind increases to $10 \mathrm{~m} / \mathrm{s}$, discontinuities in the vertical velocity field can barely be identified throughout the simulation. These two experiments suggest that the background wind provides a hostile environment for ERC and it may prevent ERC from occurring if background wind is sufficiently strong. Then, what is the mechanism behind this negative impact of background wind on ERC? Since the impact seems to be so strong, it is helpful to know if the processes governing ERC in the quiescent environment that we found previously are still active when background wind exists. To answer this question, we examined the azimuthal-mean vertical structure of simulated TCs. Figure 4.26 shows the height-radius distribution of diabatic heating at an arbitrary time (indicated by the vertical line in Figure4.25) during the ERC period. Similar to the control run, outer rainabnd activity is also seen in the two sensitivity experiments. The difference is that in the control run the outer rainband heating is cleanly separated from the eyewall heating by the moat; whereas in the sensitivity experiments the outer rainband is able to move closer to the eyewall as background wind increases. Although the outer rainband and eyewall heating may be still able to generate the radial discontinuity of vertical velocity, it cannot result in a clean ERC with concentric rings of tangential wind if the width of moat is sufficiently narrow. As the background wind increases to $10 \mathrm{~m} / \mathrm{s}$, the outer rainband in the low levels 
nearly mergers with the eyewall. Because of this, it is unable to generate a clean radial discontinuity of vertical velocity. Therefore, we may conclude that in an environment with background wind the basic physics governing ERC remains the same but the outer rainband is able to move closer to the eyewall. If the background wind is sufficiently strong, it may prevent ERC from occurring for two reasons. First, it leaves little room both spatially and temporally for a full development of a clean ERC. Second, as we showed previously (e.g., Figure 3.22), the subsidence induced by the eyewall heating is the strongest at the outer edge of convection and decreases radially outward. Thus, the stronger subsidence induced by the eyewall heating can efficiently suppress the development of outer rainband convection if it is too close to the eyewall. It is still unclear is why outer rainband convection can move closer to the eyewall in an environment with background wind. This is not an easy question to answer since the background wind not only can affect and reorganize outer rainband convection but also may impose a substantial impact on TC inner core structure. We will leave this question for our future study.

The sensitivity experiments performed here suggest that ERCs are unlikely to happen in an environment with strong background wind. Yet ERCs are frequently observed in real TCs as confirmed by satellite observations. It would be scientifically naïve if one would speculate that most of the observed ERCs might simply occur with weak background wind. To clarify this issue, we performed additional two experiments on the initial vortex with a large PV skirt with the background wind prescribed as 5 and $10 \mathrm{~m} / \mathrm{s}$. Figure 4.27 compares the vertical velocity at $5 \mathrm{~km}$ and tangential wind at $1 \mathrm{~km}$ with and without background wind. Surprisingly, the background wind does not appear to have an 
effect on ERC at all. Although there are differences in details, all three simulations show a clean ERC with well-defined concentric rings of tangential wind, which is completely different from the previous set of experiments with a smaller PV skirt in which background winds nearly destroyed ERC. These two sets of experiments on background wind suggest that the impact of background wind on ERC depends strongly on the TC structure. Although currently we cannot provide a better understanding of the physics underlying the sensitivity of ERC to background wind shown in our experiments, our result provides a helpful guidance for further investigations on ERC in an environment with background wind both observationally and numerically. 
(a) $\mathrm{w}$ at $5 \mathrm{~km}$ (Control run)

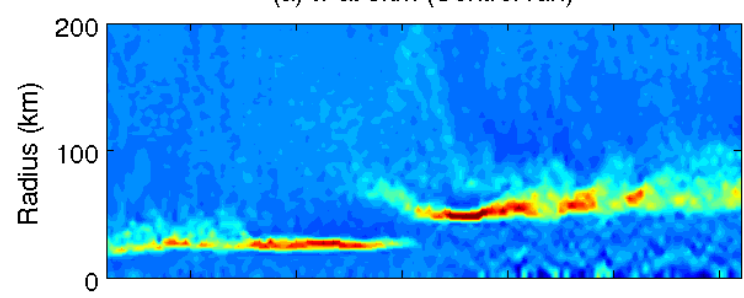

(c) $\mathrm{w}$ at $5 \mathrm{~km}$ (YSU run)

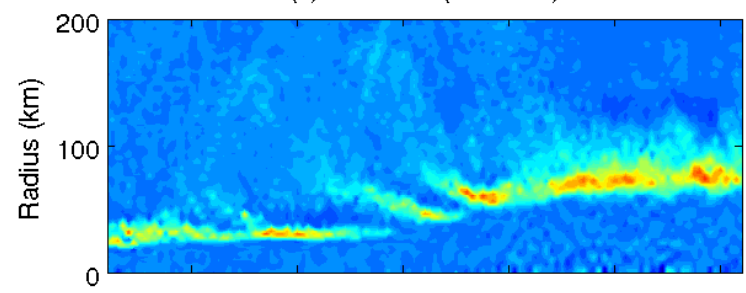

(e) w at 5km (MYNN run)

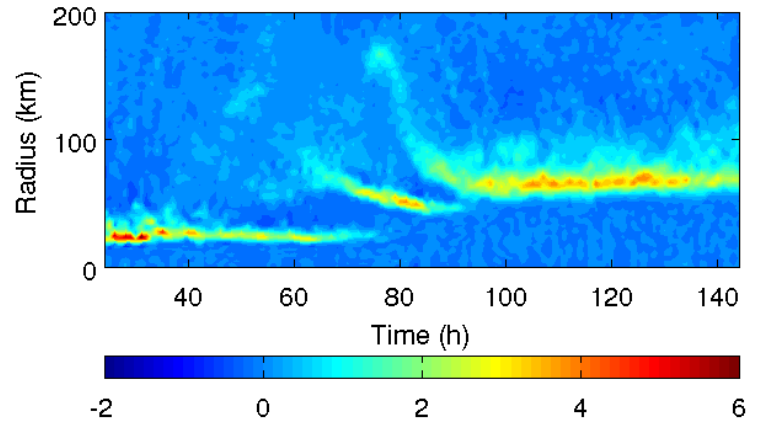

(b) Vt at $1 \mathrm{~km}$ (Control run)

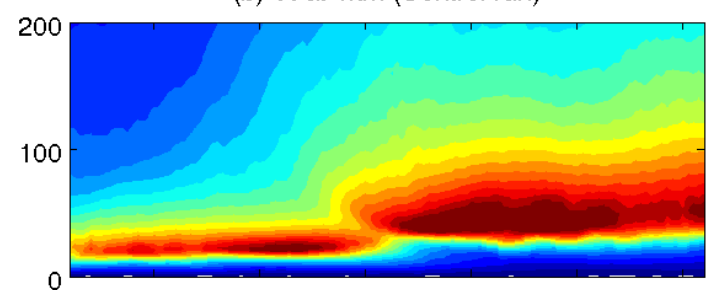

(d) Vt at $1 \mathrm{~km}$ (YSU run)

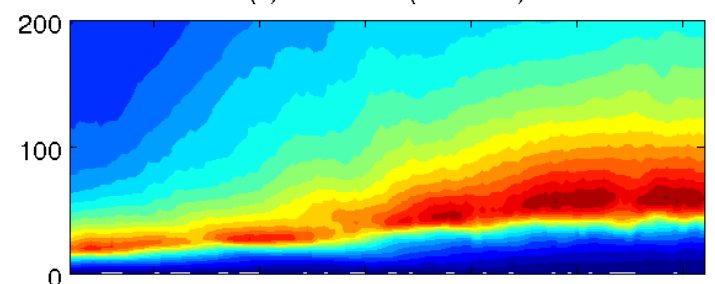

(f) Vt at $1 \mathrm{~km}$ (MYNN run)

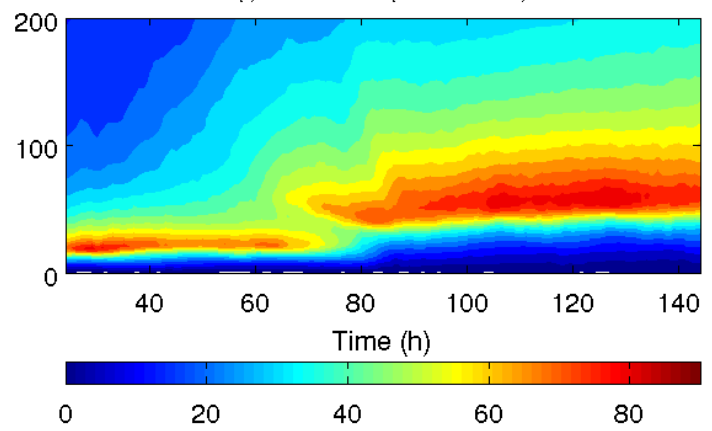

Figure4.1 Vertical velocities at $5 \mathrm{~km}$ (left column) and tangential wind at $1 \mathrm{~km}$ (right column) from the control run, and YSU and MYNN experiments, respectively. 
(a) time $=61 \mathrm{~h}$

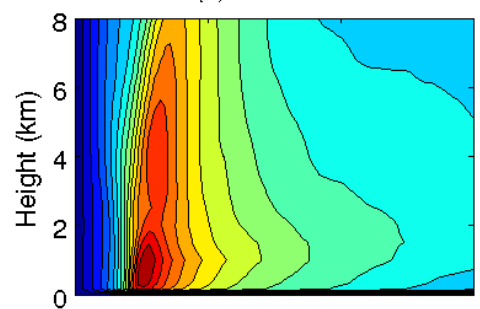

(d) time $=73 \mathrm{~h}$

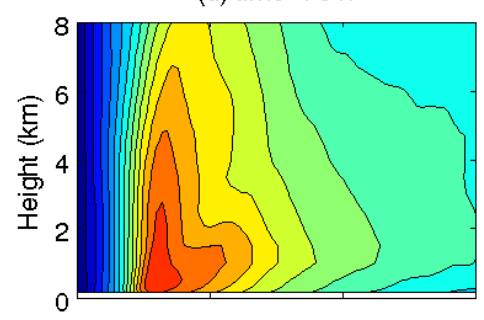

(g) time $=85 \mathrm{~h}$

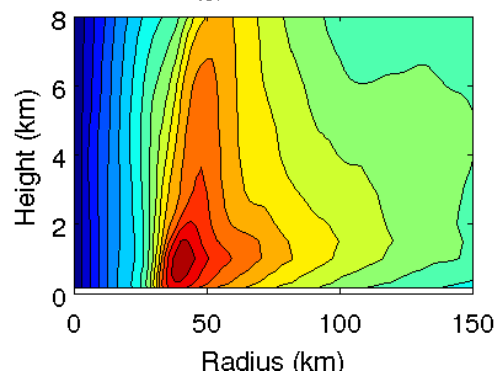

(b) time $=65 \mathrm{~h}$

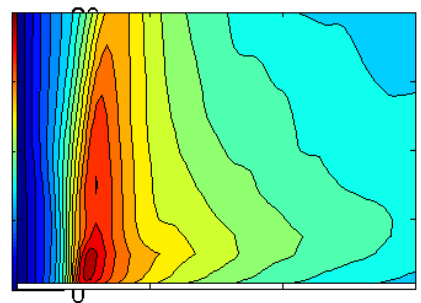

(e) time $=77 \mathrm{~h}$

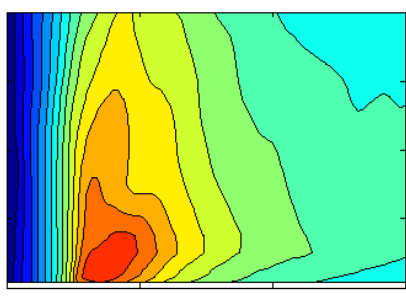

(h) time $=89 \mathrm{~h}$

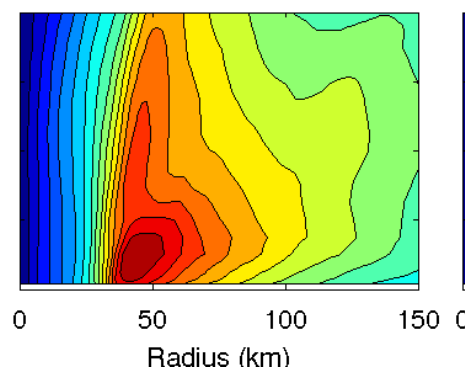

(c) time $=69 \mathrm{~h}$

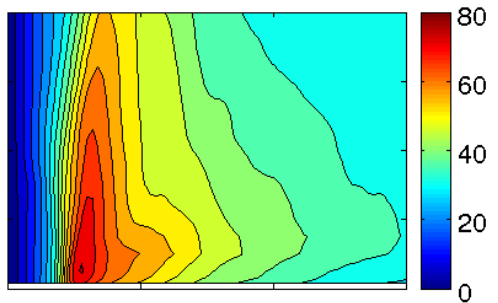

(f) time $=81 \mathrm{~h}$

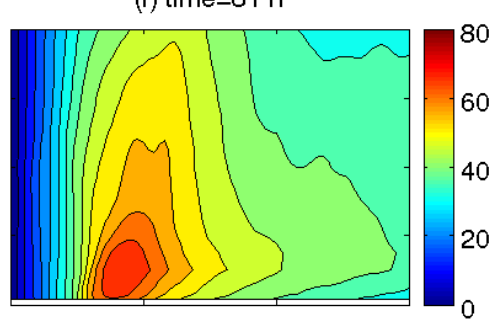

(i) time $=93 \mathrm{~h}$

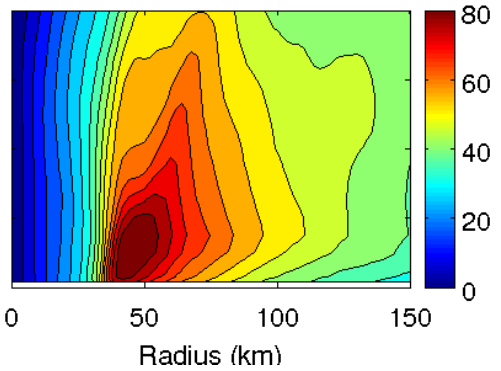

Figure 4.2 Height-radius plots of tangential wind from the YSU experiment at different times from the $61^{\text {st }} \mathrm{h}$ to $93^{\text {rd }} \mathrm{h}$. 
(a) time $=61 \mathrm{~h}$

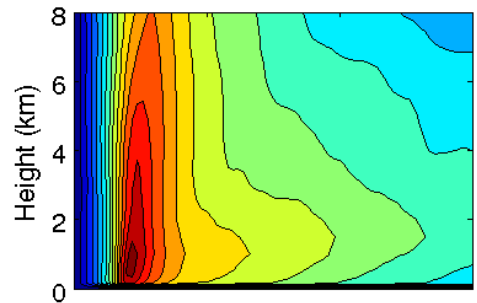

(d) time $=73 \mathrm{~h}$

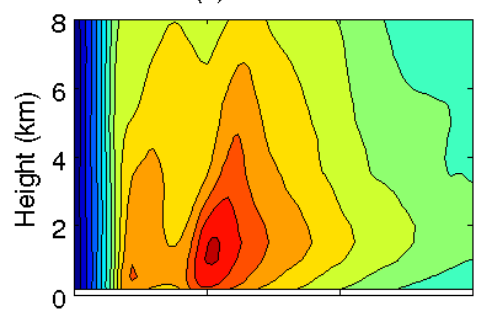

(g) time $=85 \mathrm{~h}$

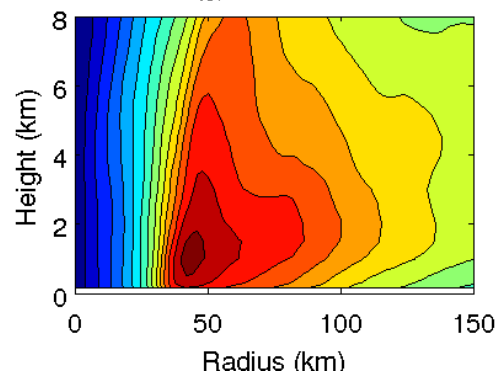

(b) time $=65 \mathrm{~h}$

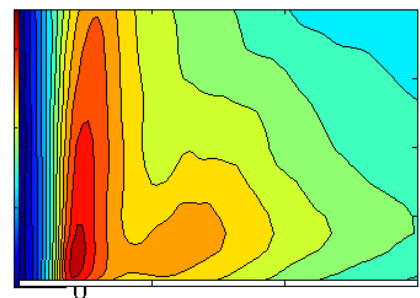

(e) time $=77 \mathrm{~h}$

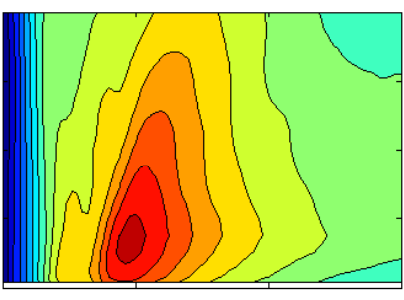

(h) time $=89 \mathrm{~h}$

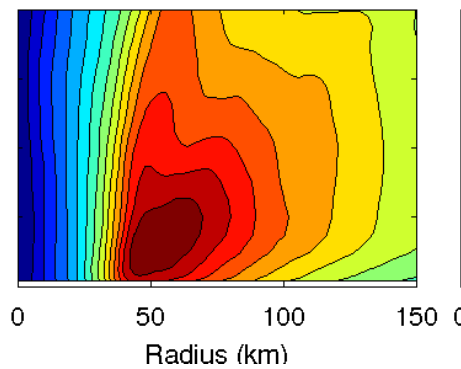

(c) time $=69 \mathrm{~h}$

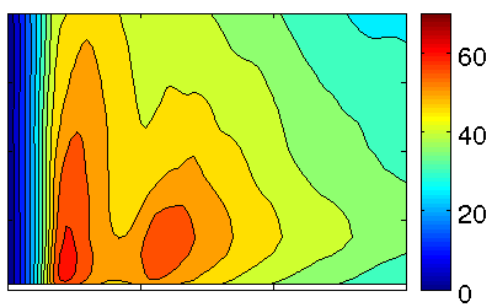

(f) time $=81 \mathrm{~h}$

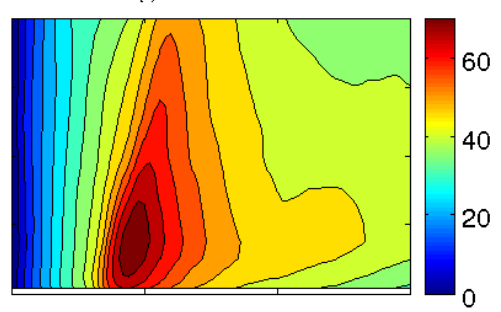

(i) time $=93 \mathrm{~h}$

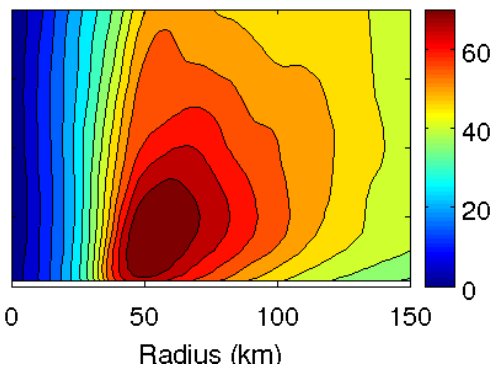

Figure 4.3 The same as Figure 4.2 but for the MYNN experiment. 
(a) MYNN time $=70 \mathrm{~h}$

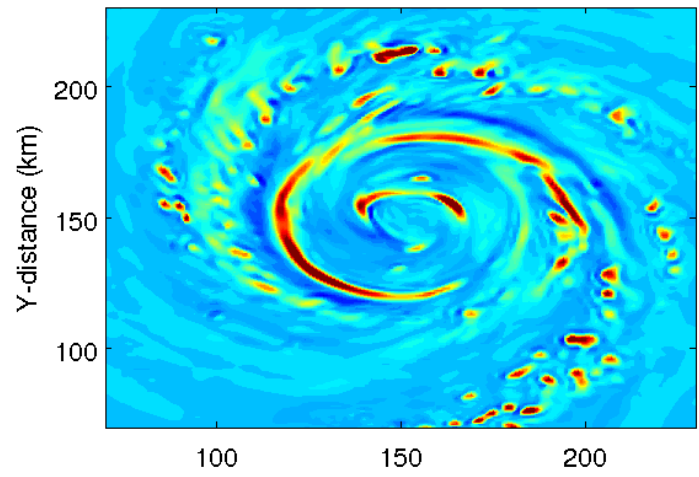

(c) YSU time $=70 \mathrm{~h}$

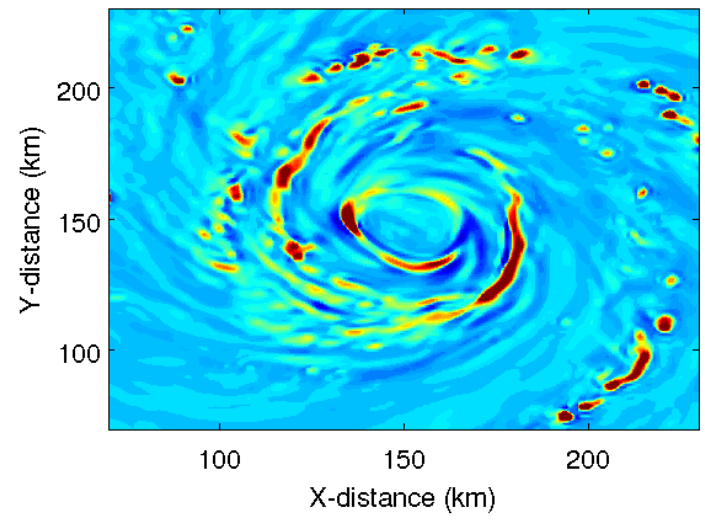

(b) MYNN time $=88 \mathrm{~h}$

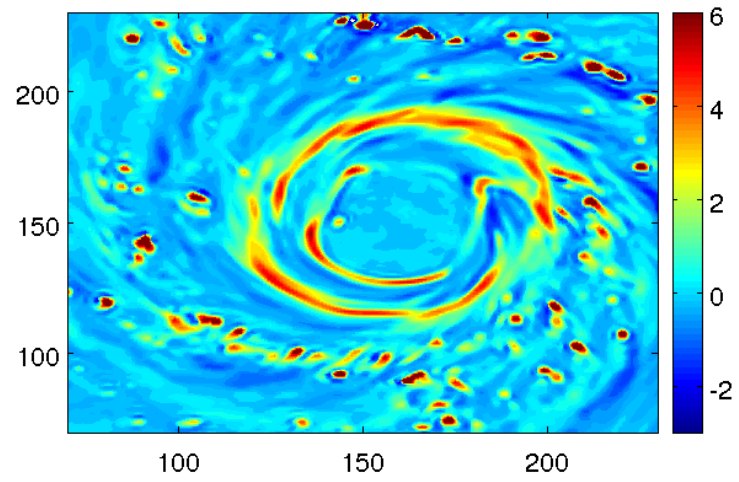

(d) YSU time $=89 \mathrm{~h}$

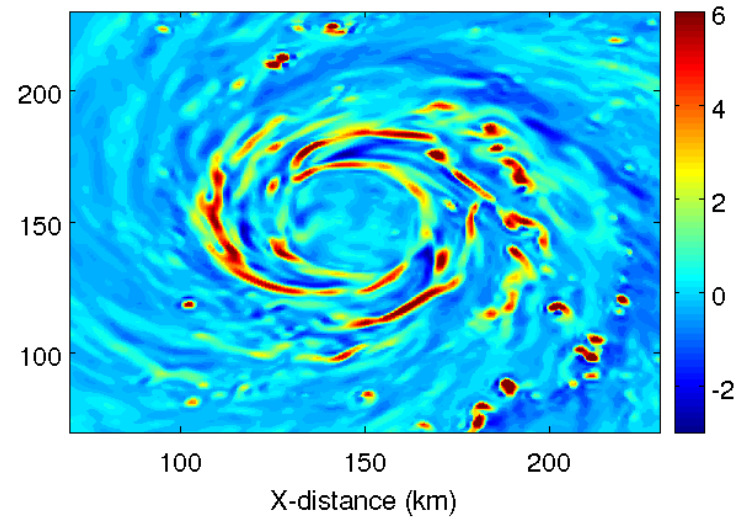

Figure 4.4 Vertical velocity at the height of $5 \mathrm{~km}$ at the two instantaneous times during the first and second outer raniband activity from the MYNN and YSU experiments. 
(a) MYNN time $=70 \mathrm{~h}$

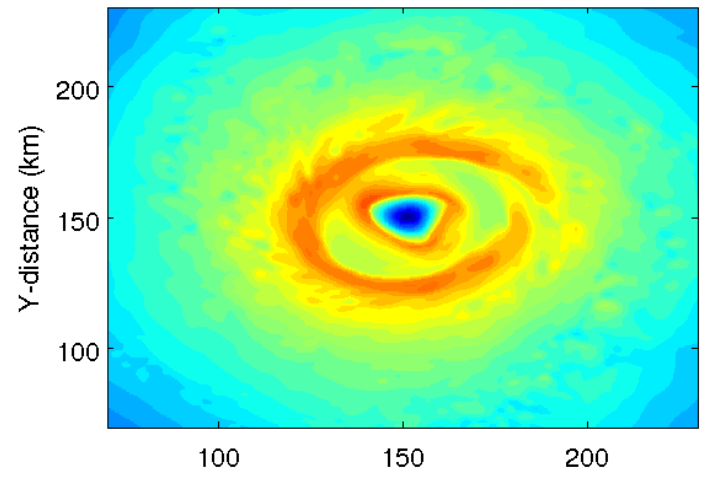

(c) YSU time $=70 \mathrm{~h}$

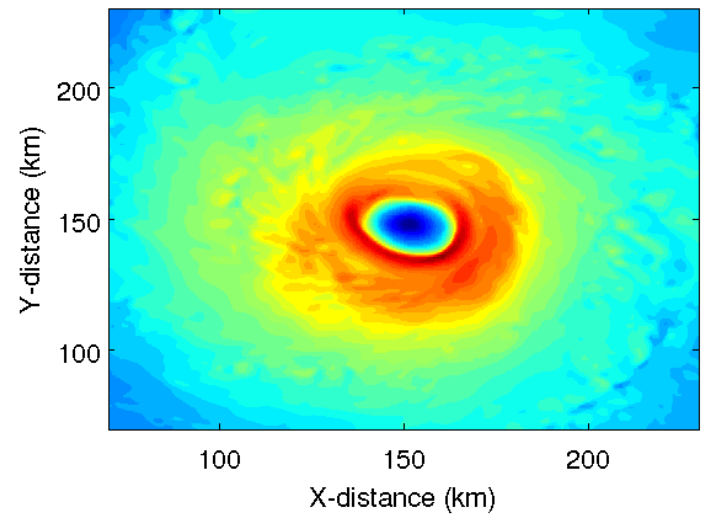

(b) MYNN time $=88 \mathrm{~h}$

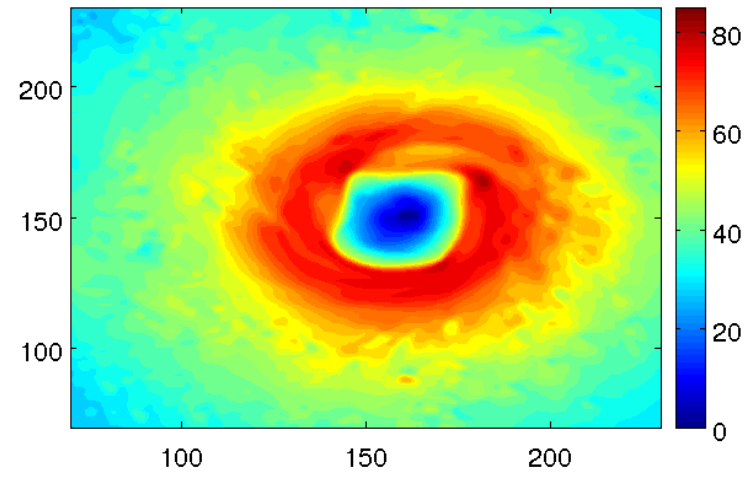

(d) YSU time $=89 \mathrm{~h}$

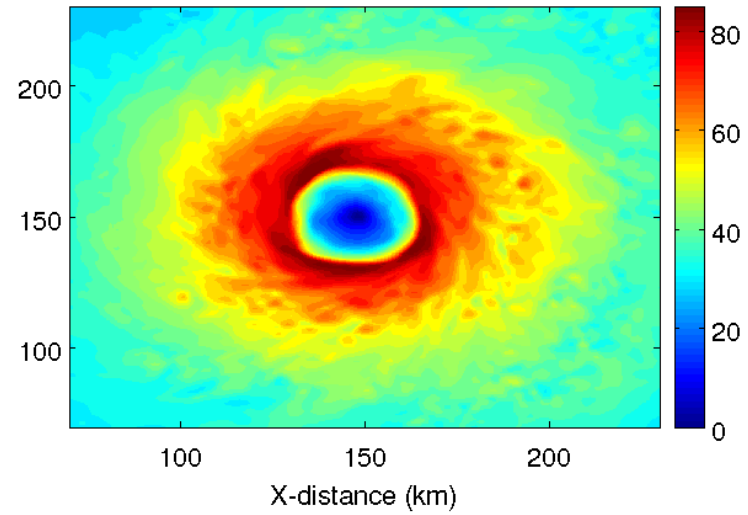

Figure 4.5 The same as Figure 4.4 but for horizontal wind speed at the height of $1 \mathrm{~km}$. 
(a) Total $\partial \mathrm{V} / \partial \mathrm{t} \times 5$

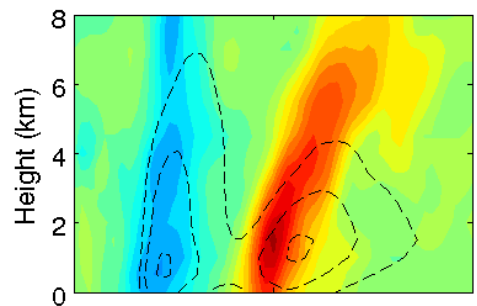

(d) $-U \zeta_{a}$

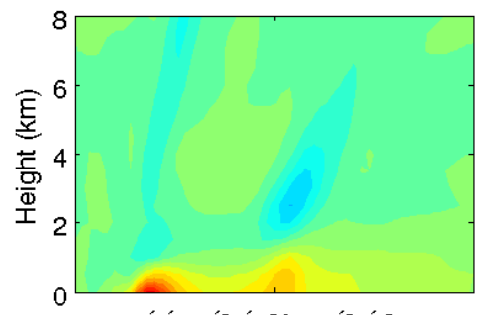

(g) $\left[-u^{\prime} \zeta_{a}^{\prime}-v^{\prime} \partial v^{\prime} / r \partial \lambda-w^{\prime} \partial v^{\prime} / \partial z\right] \times 5$

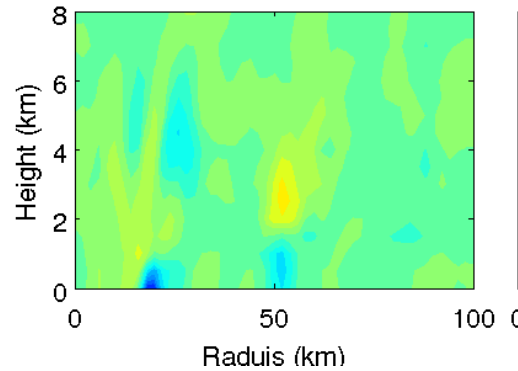

(b) Resolved $\partial \mathrm{V} / \partial \mathrm{t}$

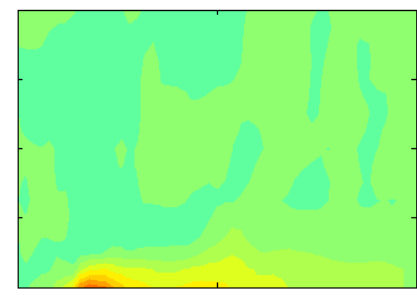

(e) $-W \partial V / \partial z$

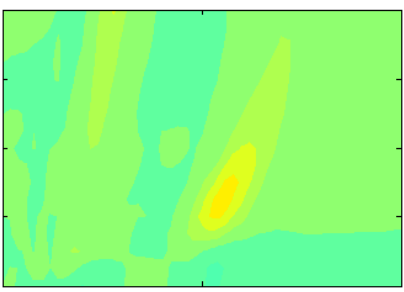

(h) SGS vertical difusion

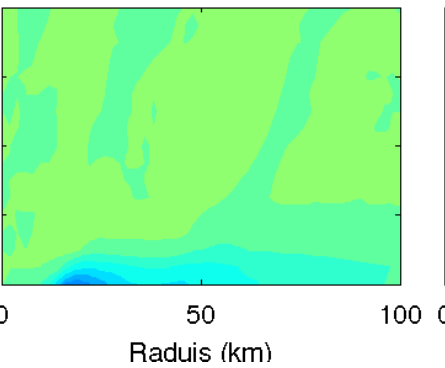

(c) SGS $\partial V / \partial t$

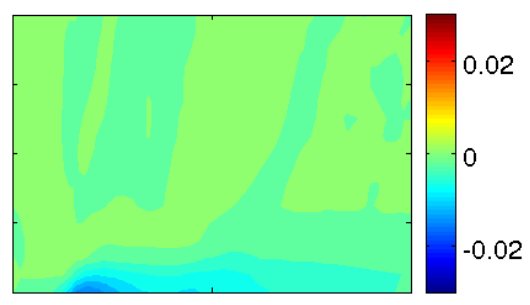

(f) $-U \zeta_{a}-W \partial V / \partial z$

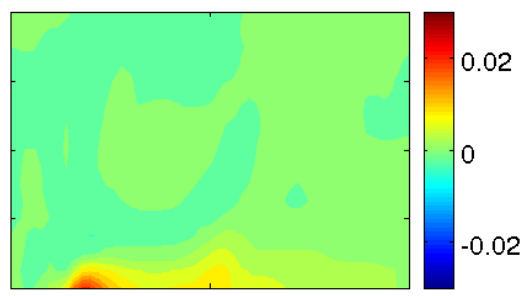

(i) SGS horizontal difusion $X 20$

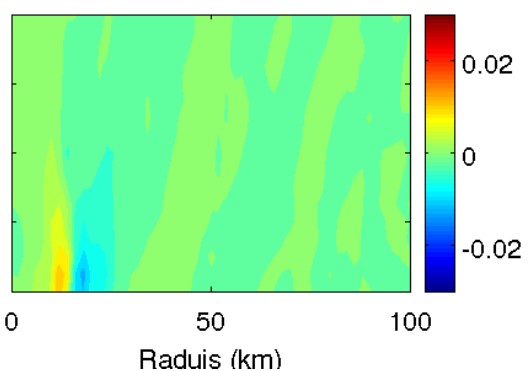

Figure 4.6 Tangential wind budget analyses (the same as Figure 3.9 but for the MYNN experiment) averaged over the period from the $70^{\text {th }} \mathrm{h}$ to $72^{\text {nd }} h$. (a): Net tendency from the sum of all terms. It has been multiplied by a factor of 5. Black dashed contours are the azimuthal-mean tangential wind; (b): Tendency from the sum of all resolved terms (i.e., $-\bar{u} \bar{\zeta}_{a}-\bar{w} \frac{\partial \bar{v}}{\partial z}-\overline{u^{\prime} \varsigma_{a}^{\prime}}-\overline{v^{\prime} \frac{\partial v^{\prime}}{r \partial \lambda}}-\overline{w^{\prime} \frac{\partial v^{\prime}}{\partial z}}$ ); (c): Tendency from the SGS processes; (d): Tendency from $-\bar{u} \bar{\zeta}_{a} ;(\mathrm{e})$ : Tendency from $-\bar{w} \frac{\partial \bar{v}}{\partial z}$; (f): Tendency from $-\bar{u} \bar{\zeta}_{a}-\bar{w} \frac{\partial \bar{v}}{\partial z} ;(\mathrm{g})$ Tendency from the resolved asymmetric eddies, $-\overline{u^{\prime} \varsigma_{a}^{\prime}}-\overline{v^{\prime} \frac{\partial v^{\prime}}{r \partial \lambda}}-\overline{w^{\prime} \frac{\partial v^{\prime}}{\partial z}}$. It has been multiplied by a 
factor of 5; (h): Tendency from the SGS vertical turbulent mixing; (i): Tendency from the SGS horizontal turbulent mixing. It has been multiplied by a factor of 20 . 
(a) Total JV/at at $61 \mathrm{~h}$

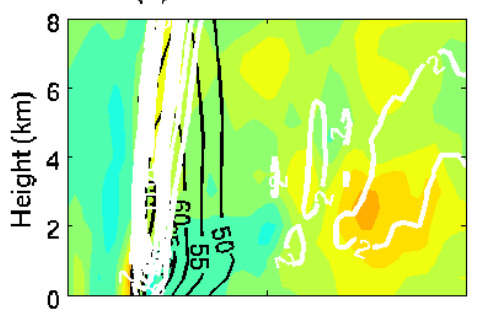

(d) Total JV/at at $73 \mathrm{~h}$

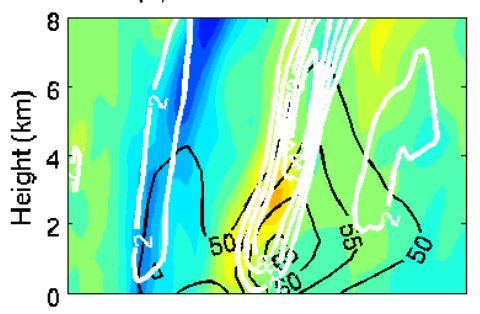

(g) Total av/2t at $85 \mathrm{~h}$

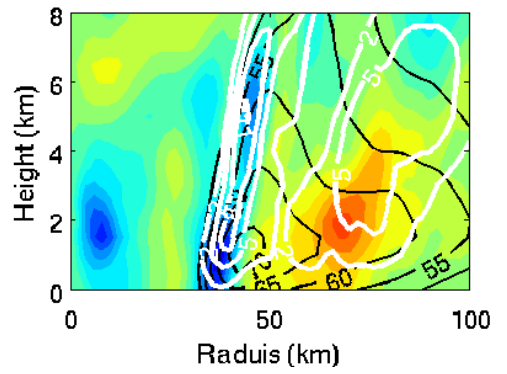

(b) Total aV/at at $65 \mathrm{~h}$

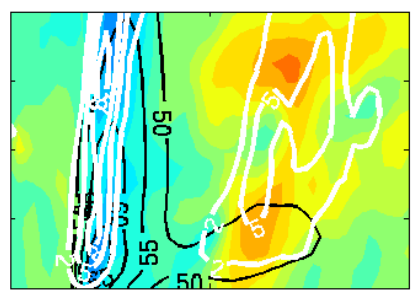

(e) Total av/at at $77 \mathrm{~h}$

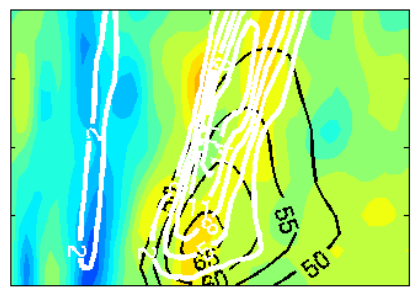

(h) Total av/at at $89 \mathrm{~h}$

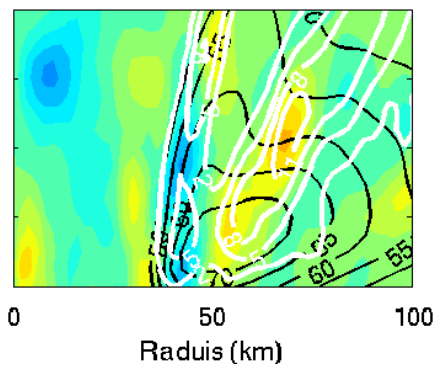

(c) Total av/at at $69 \mathrm{~h}$

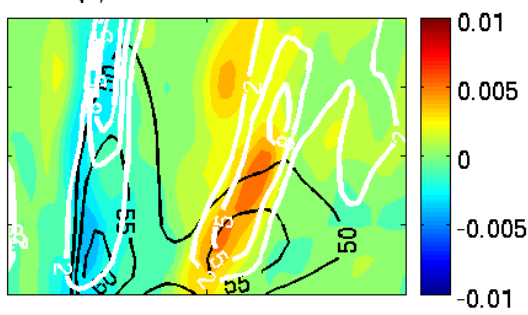

(f) Total aV/at at $81 \mathrm{~h}$

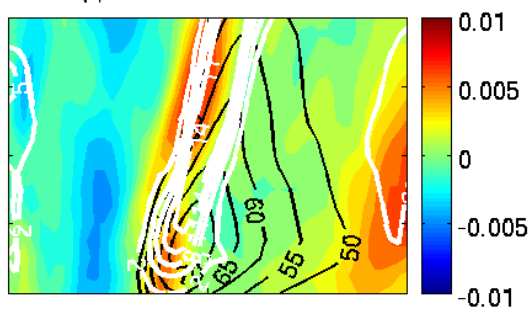

(i) Total av/at at $93 \mathrm{~h}$

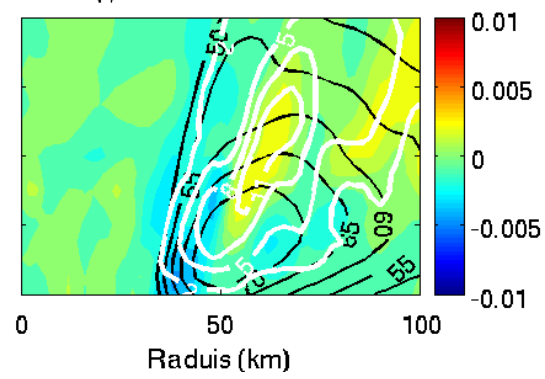

Figure 4.7 Height-radius plot of azimuthal-mean diabatic heating (white contours), tangential wind (black contours), and net tendency of tangential wind at different times in the ERC period from the MYNN experiment. Note that the diabatic heating has been multiplied by a factor of 1000 . The contour starts from 2 with an increment interval of 3 . 


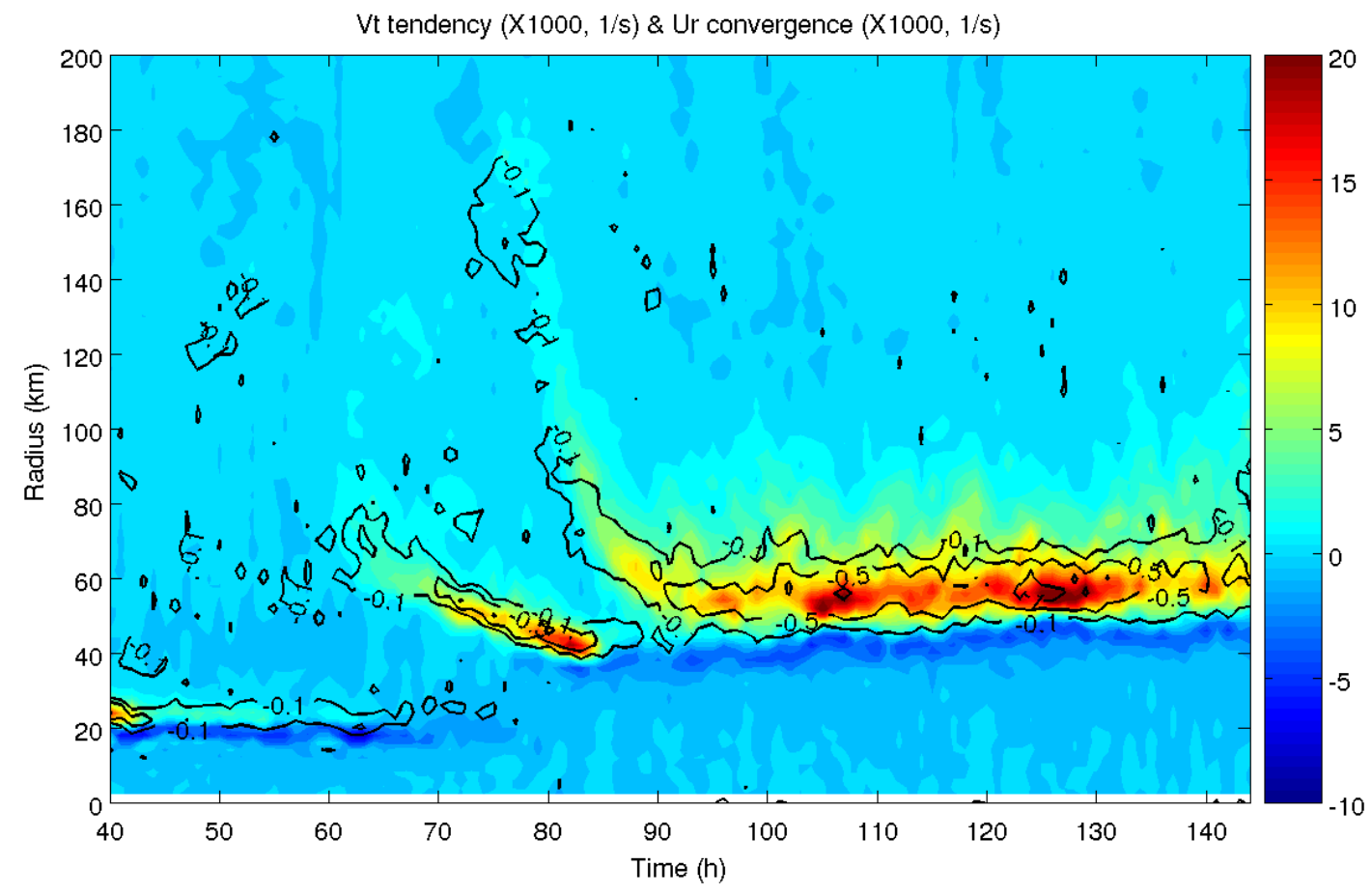

Figure 4.8 Tendency of tangential wind induced by the horizontal advection of absolute vorticity and vertical advection of tangential wind (color shades) and convergence of radial inflow (black contours with interval $-1,-0.5$, and $-0.11 / \mathrm{s}$ ) at the height of $1 \mathrm{~km}$ from the MYNN experiment. Note that both tangential wind tendency and convergence of radial inflow have been multiplied by a factor of 1000. 
(a) Relative humidity (\%) at $300 \mathrm{~m}$

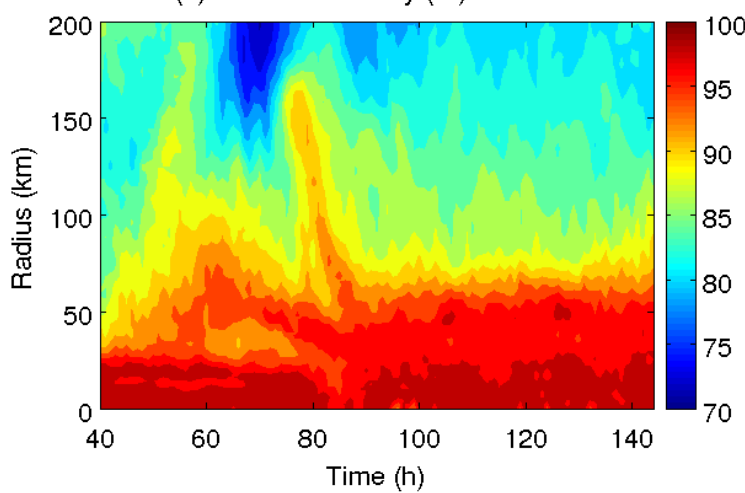

(c) Surface buoyancy flux $(\mathrm{W} / \mathrm{m} 2)$

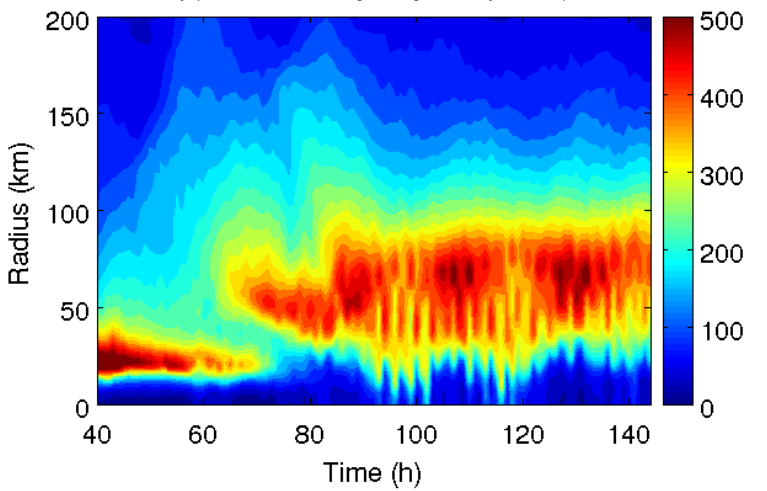

(b) $\theta_{\mathrm{e}}(\mathrm{K})$ at $300 \mathrm{~m}$

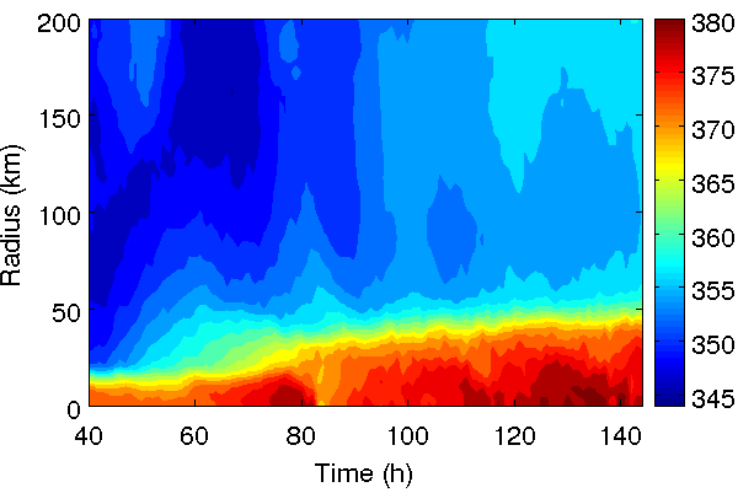

(d) Cloud mixing ratio $(\mathrm{g} / \mathrm{kg})$ over the radii of $60-100 \mathrm{~km}$

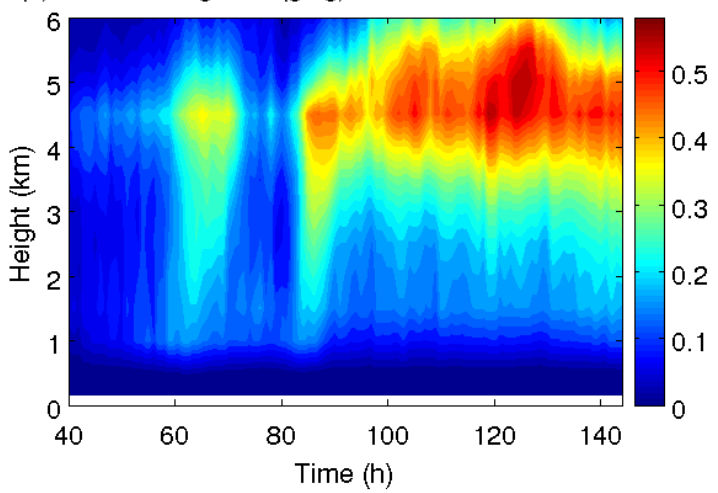

Figure 4.9 Time evolution of (a) relative humidity at the height of $300 \mathrm{~m}$, (b) equivalent potential temperature at the height of $300 \mathrm{~m}$, (c) surface buoyancy fluxes, and (d) cloud water mixing ratio averaged over the radii of $60-100 \mathrm{~km}$. 
(a) Heating at $70 \mathrm{~h}$

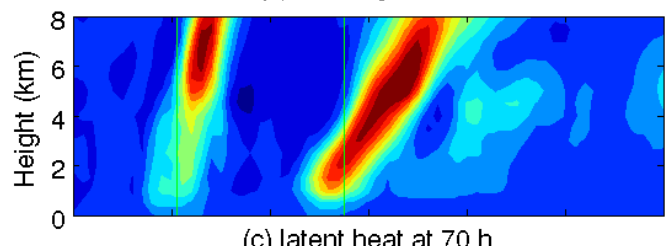

(c) latent heat at $70 \mathrm{~h}$

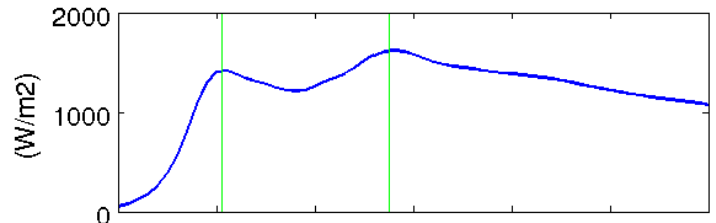

(e) $10-\mathrm{m}$ wind speed at $70 \mathrm{~h}$

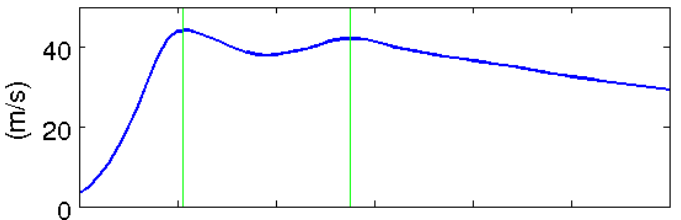

(g) friction velocity at $70 \mathrm{~h}$

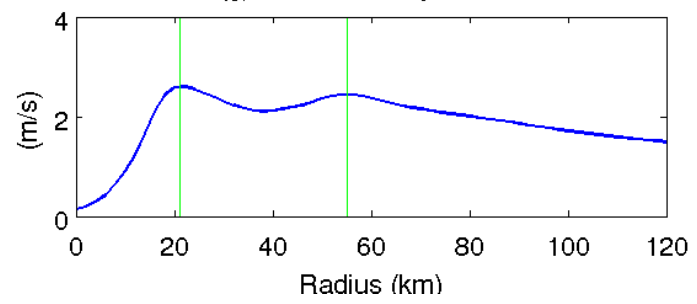

(b) Heating at $85 \mathrm{~h}$
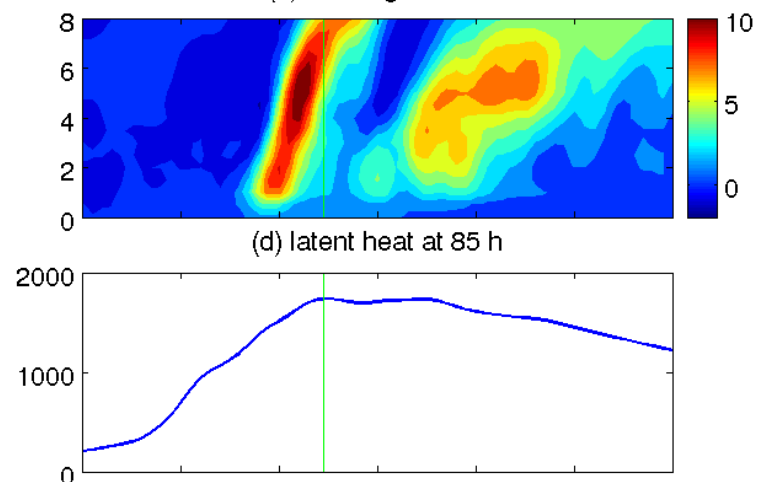

(f) 10-m wind speed at $85 \mathrm{~h}$

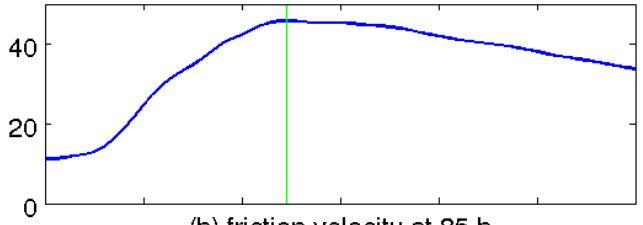

(h) friction velocity at $85 \mathrm{~h}$

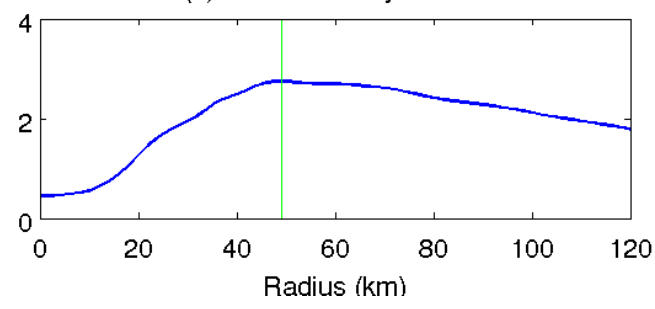

Figure 4.10 Diabatic heating, surface latent heat fluxes, 10-m wind speed, and friction velocity at the $70^{\text {th }} \mathrm{h}$ and $85^{\text {th }} \mathrm{h}$ respectively from the MYNN experiment. 
(a) Diabatic heating and $w$ at $65 \mathrm{~h}$

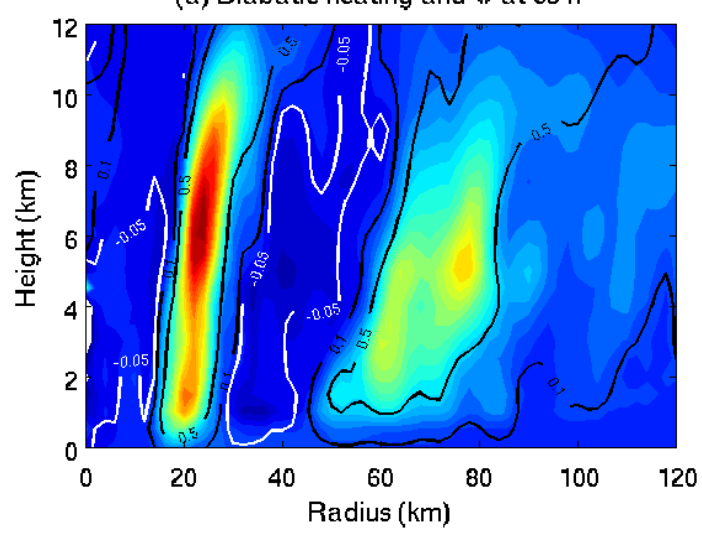

(b) Diabatic heating and $w$ at $85 \mathrm{~h}$

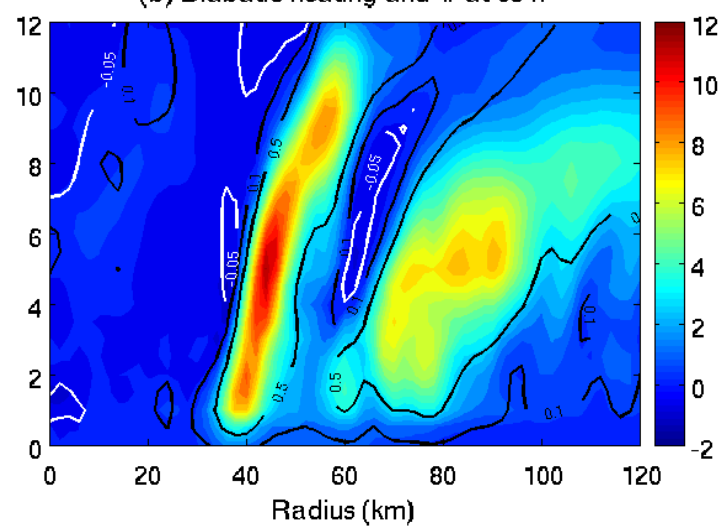

Figure 4.11 Diabatic heating (color shades) and vertical velocity (contours) at the $65^{\text {th }}$ $\mathrm{h}$ and $85^{\text {th }} \mathrm{h}$ from the MYNN experiment where updraft and downdraft are indicated by black $(0.1$ and $0.5 \mathrm{~m} / \mathrm{s})$ and white contours $(-0.05 \mathrm{~m} / \mathrm{s})$, respectively. 
(a) Radial flow at $65 \mathrm{~h}$

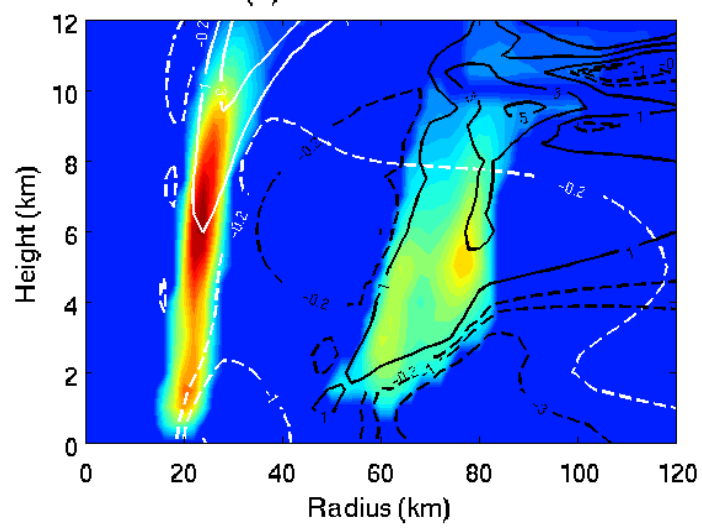

(b) Radial flow at $85 \mathrm{~h}$

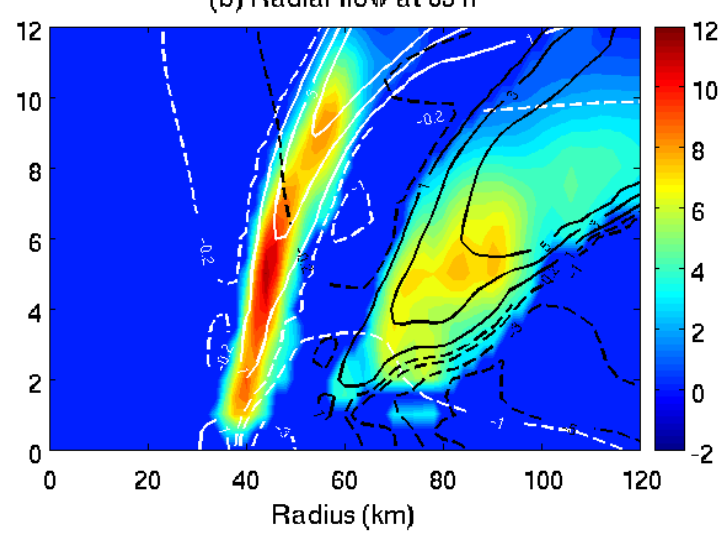

Figure 4.12 Radial flow induced by the eyewall and outer rainband heating at the $65^{\text {th }} \mathrm{h}$ and $85^{\text {th }} \mathrm{h}$ from the MYNN experiment where the radial inflow and outflow induced by the eyewall heating are indicated by solid and dashed white contours and those induced by the outer rainband heating are indicated by solid and dashed black contours, respectively. The contour values of inflow and outflow are $-0.2,-1$, and 1,3 , and $5 \mathrm{~m} / \mathrm{s}$, respectively. 
(a) Heating at $75 \mathrm{~h}$

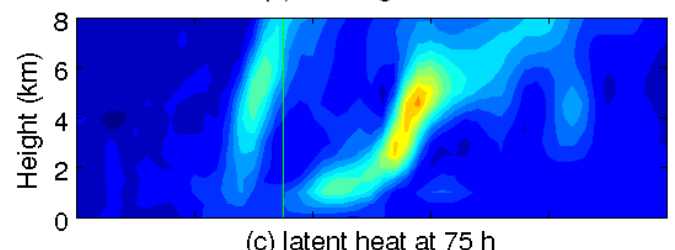

(c) latent heat at $75 \mathrm{~h}$

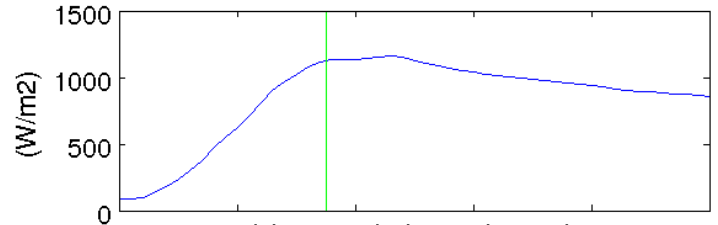

(e) $10-\mathrm{m}$ wind speed at $75 \mathrm{~h}$

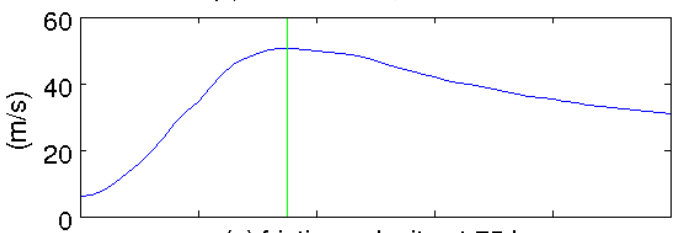

(g) friction velocity at $75 \mathrm{~h}$

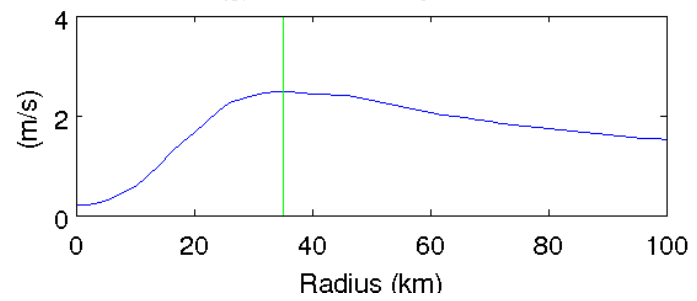

(b) Heating at $89 \mathrm{~h}$
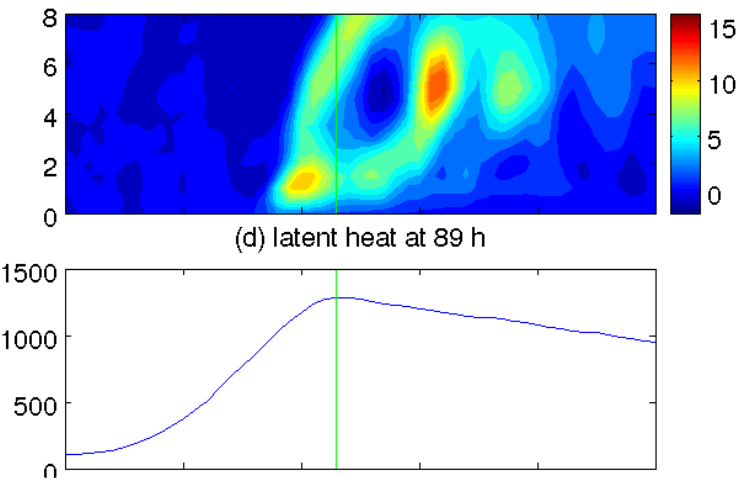

(f) 10-m wind speed at $89 \mathrm{~h}$

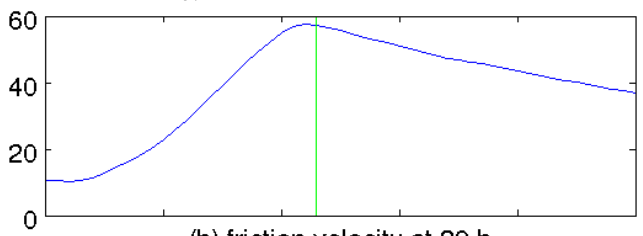

(h) friction velocity at $89 \mathrm{~h}$

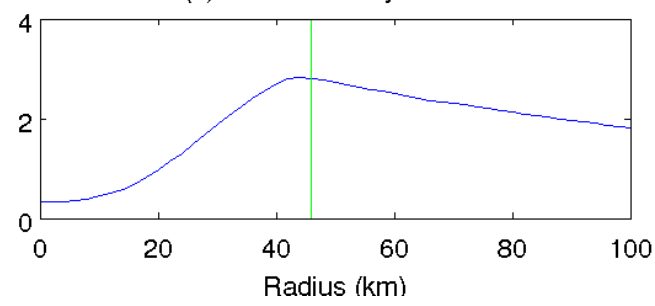

Figure 4.13 Diabatic heating, surface latent heat fluxes, $10-\mathrm{m}$ wind speed, and friction velocity at the $75^{\text {th }} \mathrm{h}$ and $85^{\text {th }} \mathrm{h}$ respectively from the YSU experiment. 
(a) Heating at $70 \mathrm{~h}$, MYNN

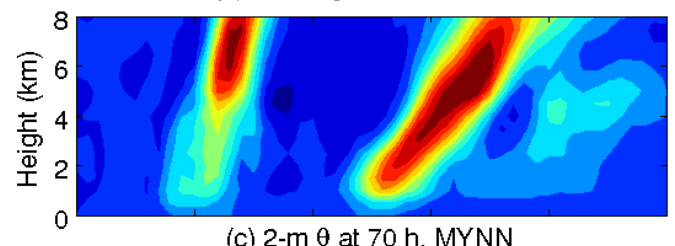

(c) 2-m $\theta$ at $70 \mathrm{~h}, \mathrm{MYNN}$

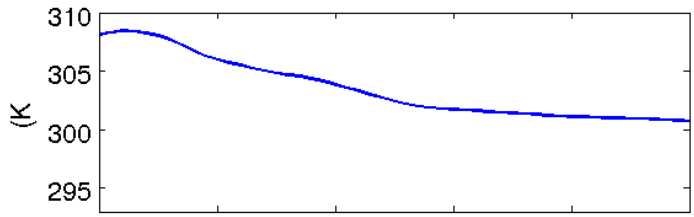

(e) Heating at $75 \mathrm{~h}, \mathrm{YSU}$

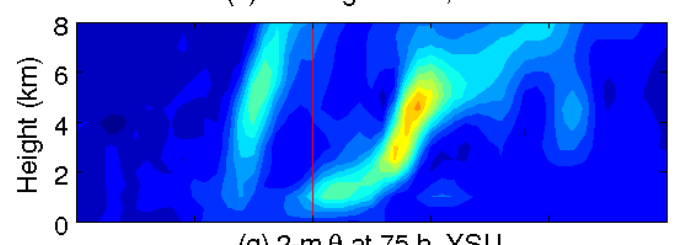

(g) 2-m $\theta$ at $75 \mathrm{~h}, \mathrm{YSU}$

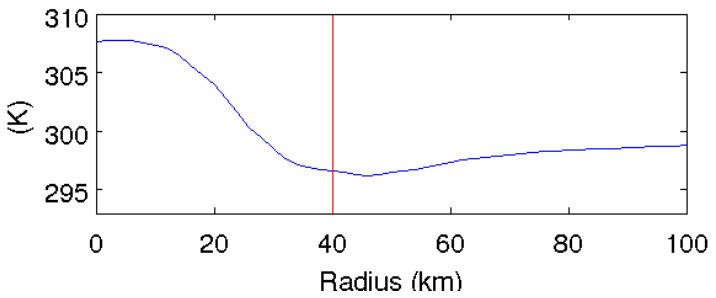

(b) Heating at $85 \mathrm{~h}$, MYNN
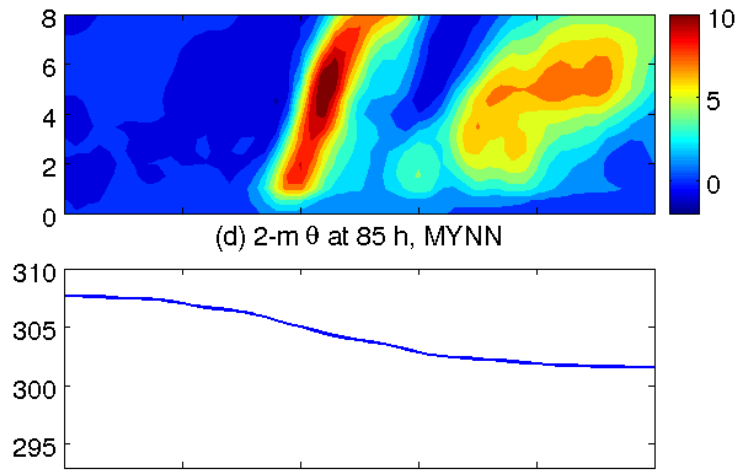

(f) Heating at $89 \mathrm{~h}$

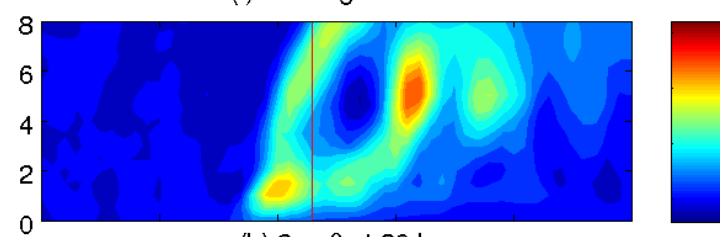

(h) $2-\mathrm{m} \theta$ at $89 \mathrm{~h}$

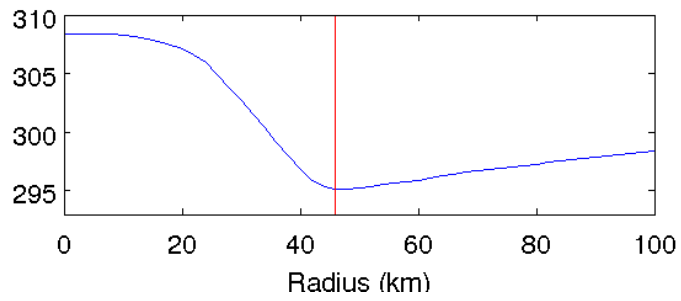

Figure 4.14 Radial distribution of diabatic heating and 2-m potential temperature during ERC events from the MYNN and YSU experiments. 
(a) $w$ at $5 \mathrm{~km}$ (Cont)

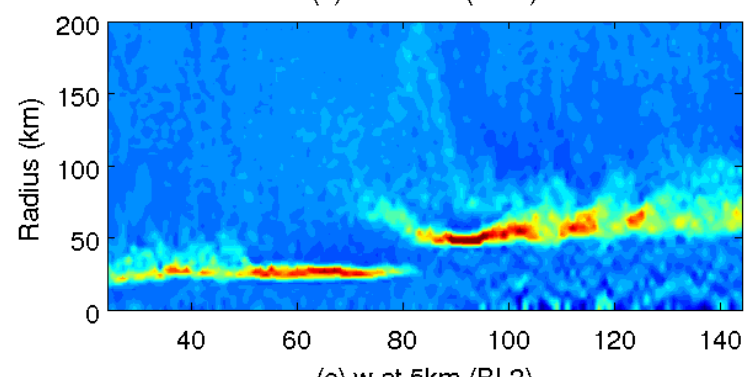

(c) $w$ at $5 \mathrm{~km}$ (BL2)

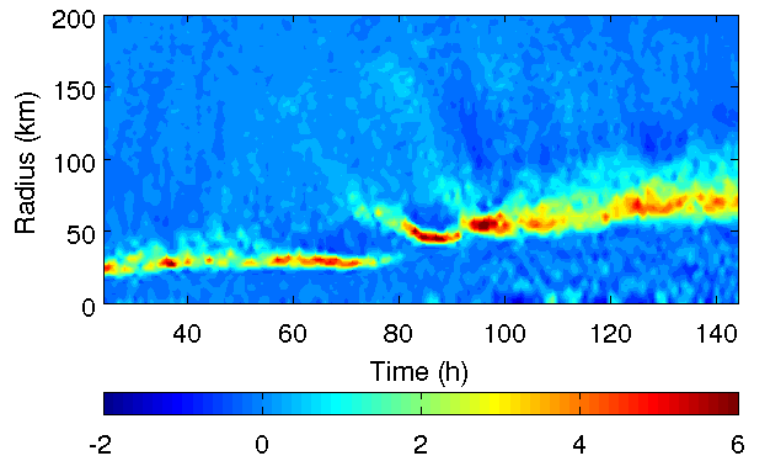

(b) Vt at $1 \mathrm{~km}$ (Cont)

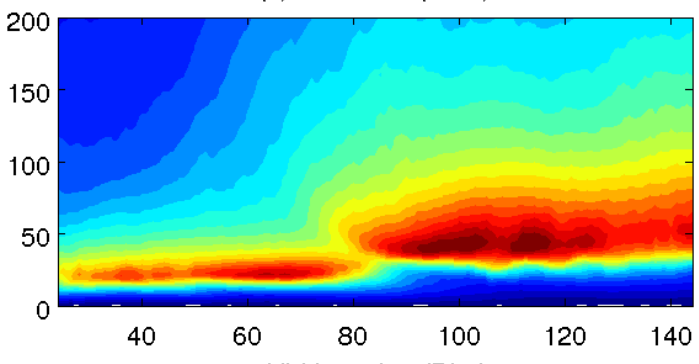

(d) $\mathrm{Vt}$ at $1 \mathrm{~km}(\mathrm{BL} 2)$

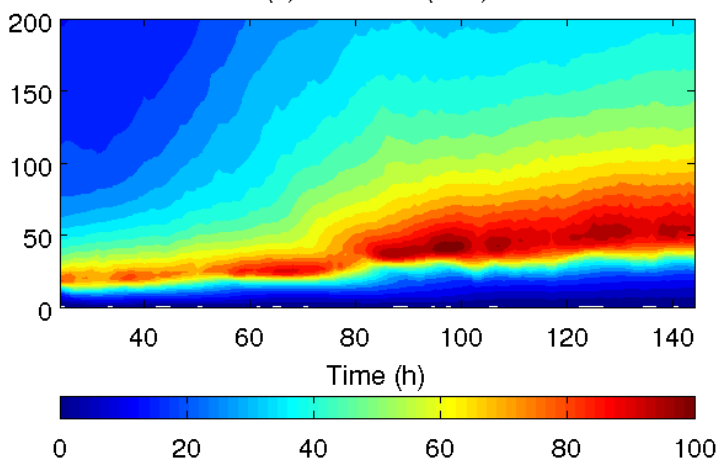

Figure 4.15 Time evolution of vertical velocity at $5 \mathrm{~km}$ and tangnetial wind at $1 \mathrm{~km}$ from the control run and MYJ-BL2 experiment. 
(a) time $=61 \mathrm{~h}$

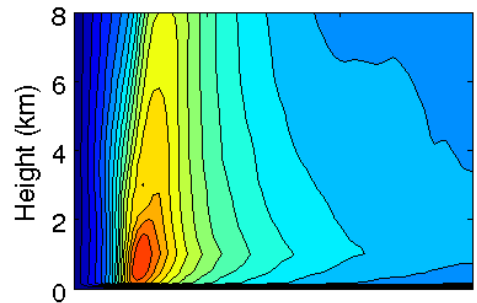

(d) time $=73 \mathrm{~h}$

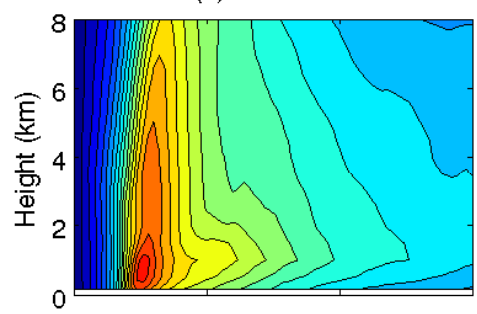

(g) time $=85 \mathrm{~h}$

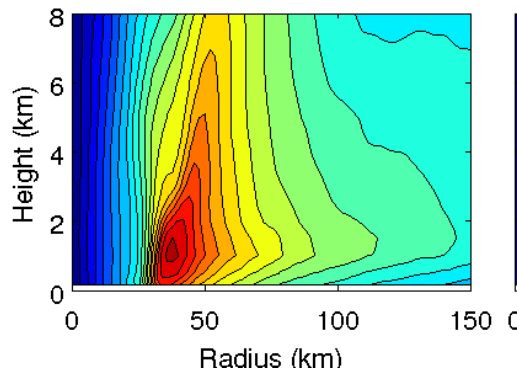

(b) time $=65 \mathrm{~h}$

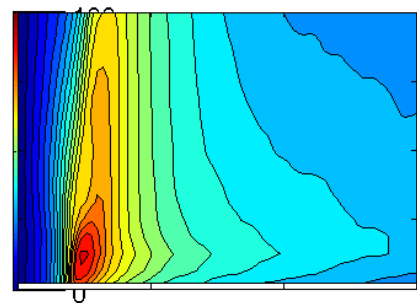

(e) time $=77 \mathrm{~h}$

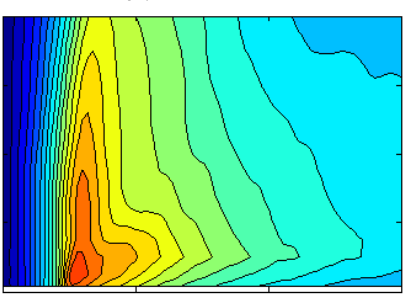

(h) time $=89 \mathrm{~h}$

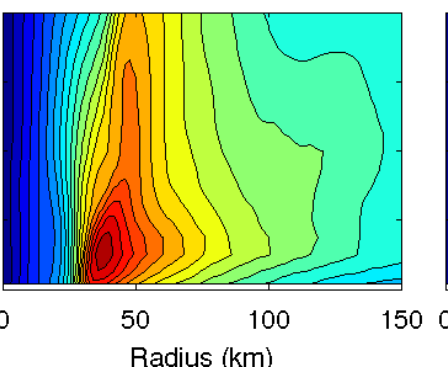

(c) time $=69 \mathrm{~h}$
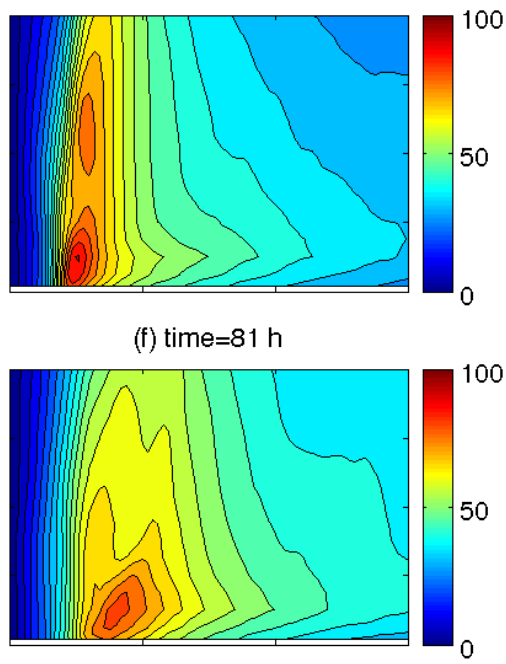

(i) time $=93 \mathrm{~h}$

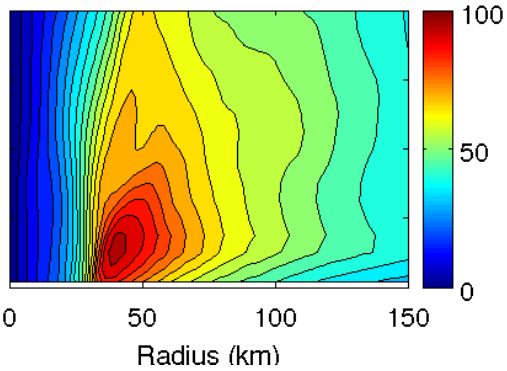

Figure 4.16 Height-radius plots of tangential wind from the MYJ-BL2 experiment at different times from the $61^{\text {st }} \mathrm{h}$ to $93^{\text {rd }} \mathrm{h}$. 
(a) Heating at $73 \mathrm{~h}$

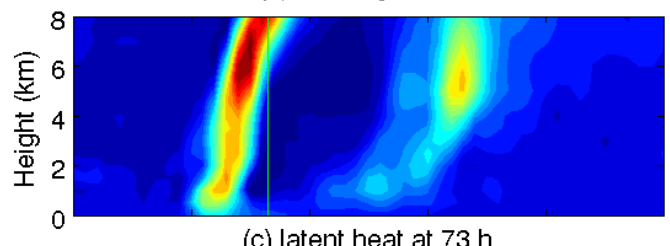

(c) latent heat at $73 \mathrm{~h}$

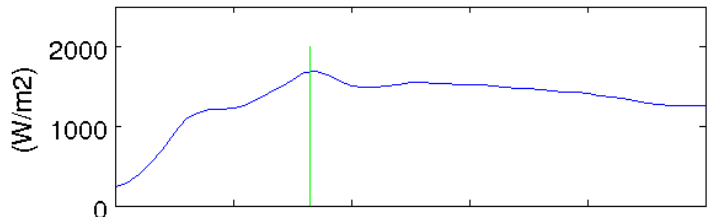

(e) $10-\mathrm{m}$ wind speed at $73 \mathrm{~h}$

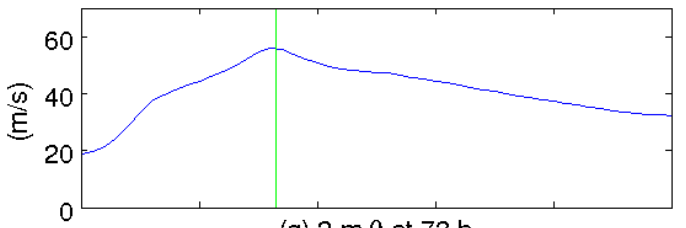

(g) $2-\mathrm{m} \theta$ at $73 \mathrm{~h}$

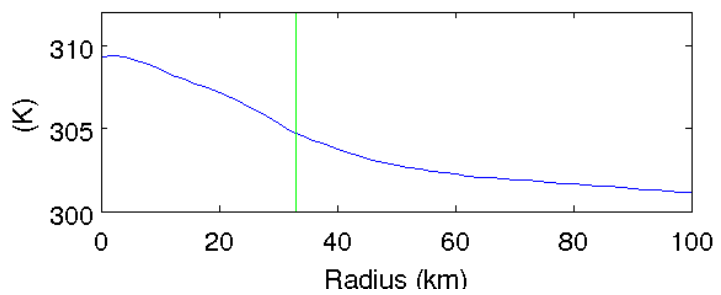

(b) Heating at $89 \mathrm{~h}$
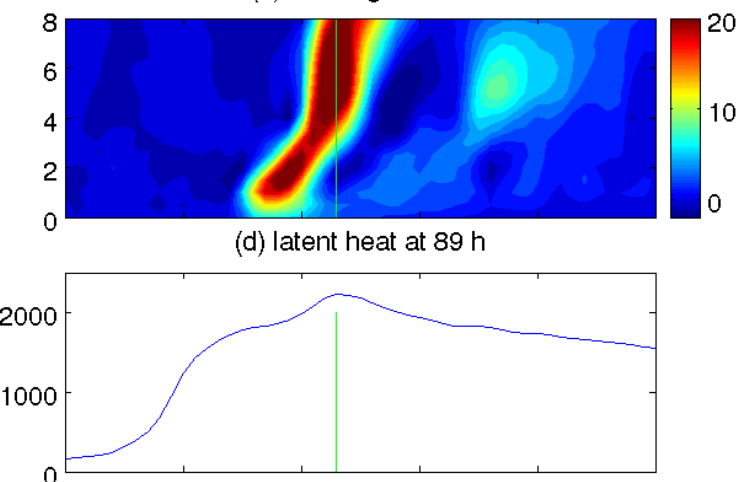

(f) 10-m wind speed at $89 \mathrm{~h}$

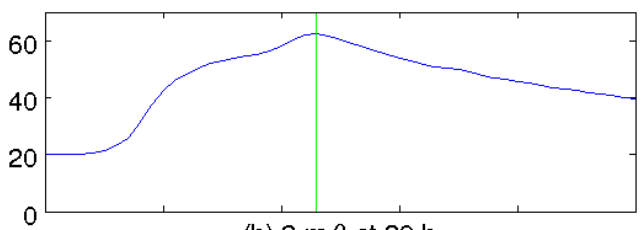

(h) $2-\mathrm{m} \theta$ at $89 \mathrm{~h}$

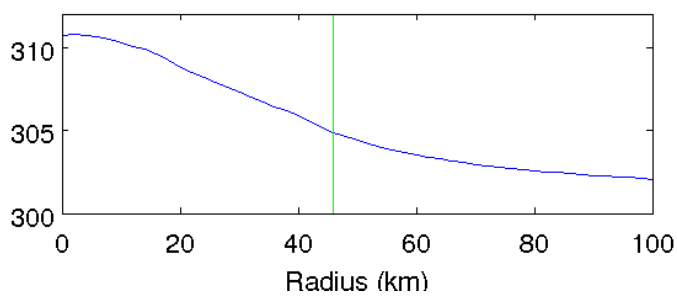

Figure 4.17 Radial distribution of diabatic heating, surface latent heat flux, 10-m wind speed, and 2-m potential temperature during ERC events from the MYJ-BL2 experiment. 


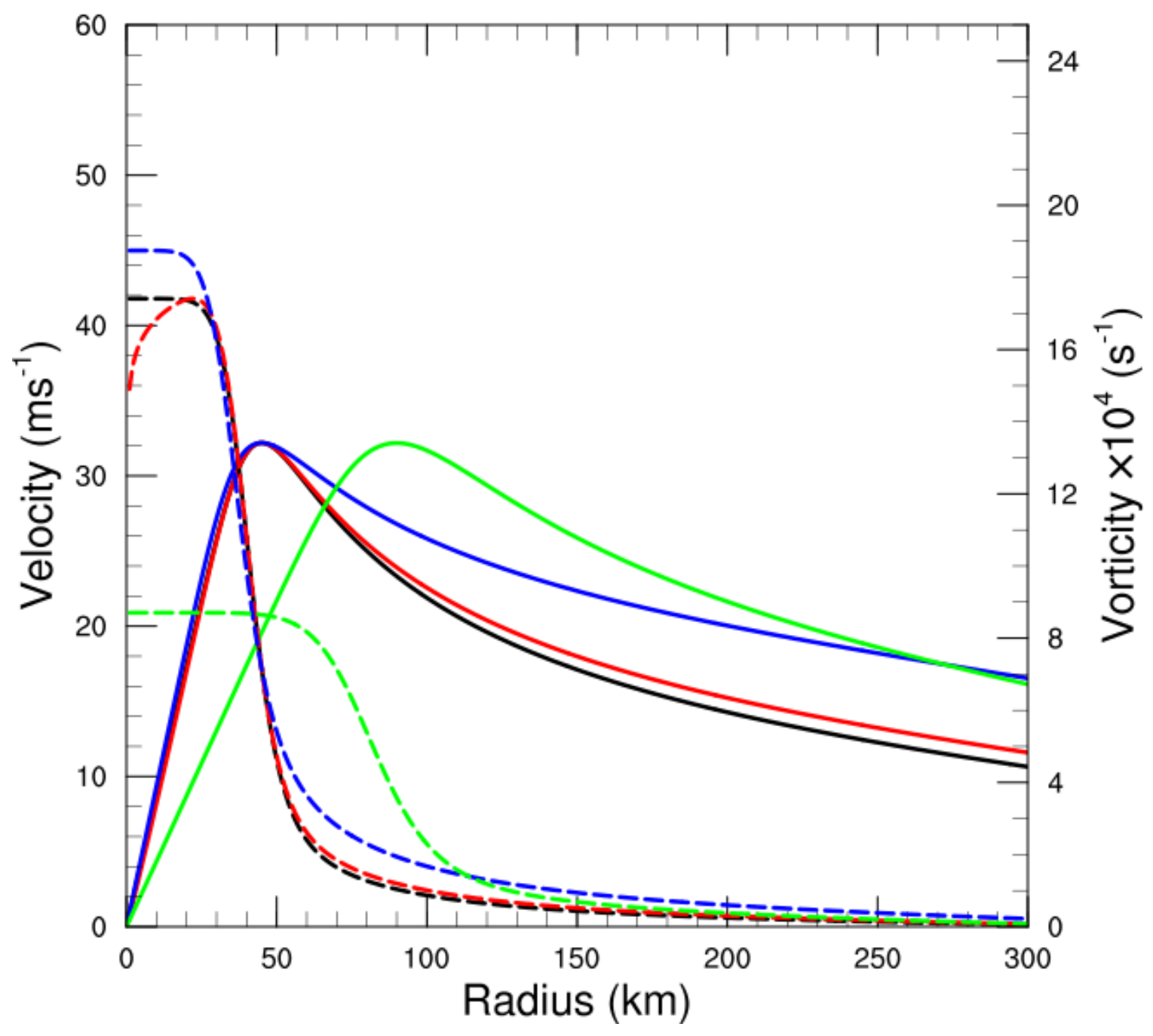

Figure 4.18 Radial profiles of tangential wind (solid) and relative vorticity (dash) for control simulation (black) and sensitivity tests with double RMAX (green), stronger tangential winds outside $\operatorname{RMAX}(\mathrm{n}=1.35$; blue) and with an annulus $\operatorname{vortex}(\mathrm{k}=1.05 ;$ red $)$. 
(a) $w$ at $5 \mathrm{~km}$ (Cont)

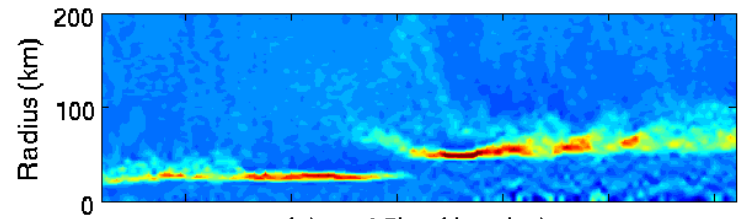

(c) $w$ at $5 \mathrm{~km}$ (Annulus)

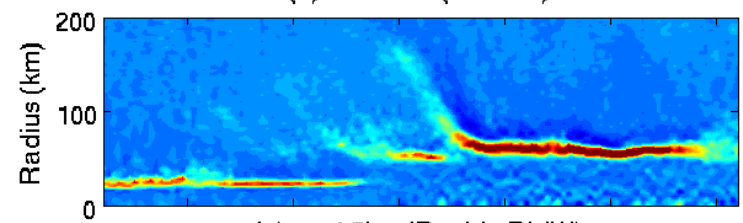

(e) $w$ at $5 \mathrm{~km}$ (Double RMW)

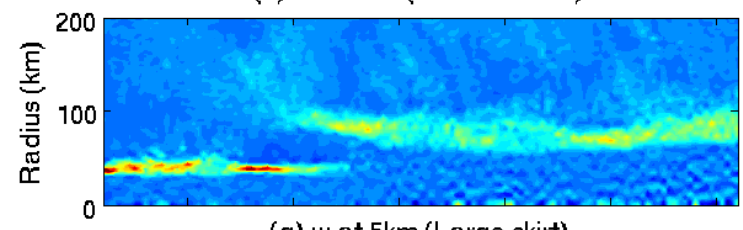

(g) w at $5 \mathrm{~km}$ (Large skirt)

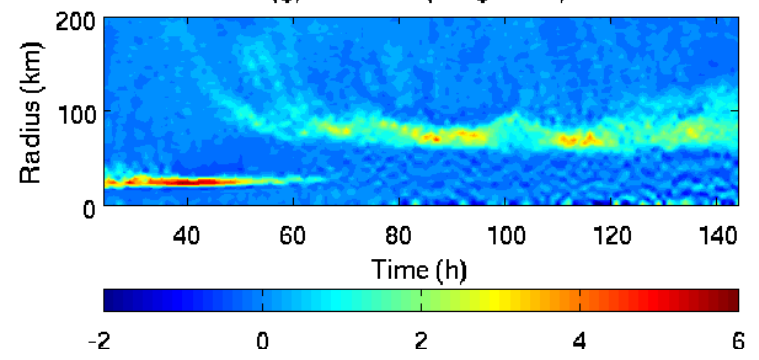

(b) Vt at $1 \mathrm{~km}$ (Cont)

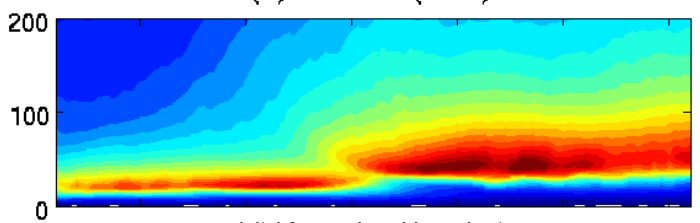

(d) Vt at $1 \mathrm{~km}$ (Annulus)

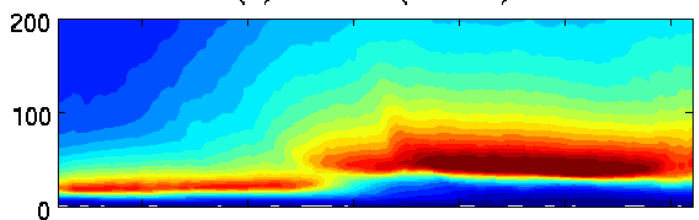

(f) Vt at $1 \mathrm{~km}$ (Double RMW)

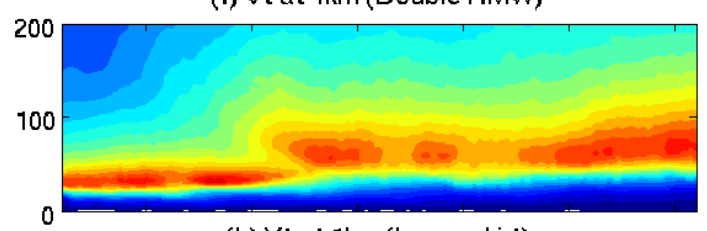

(h) Vt at $1 \mathrm{~km}$ (Large skirt)

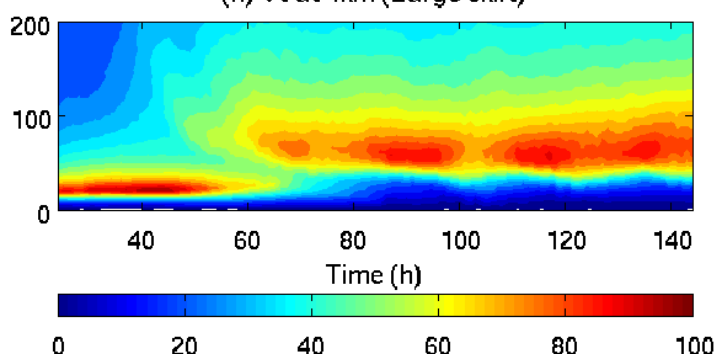

Figure 4.19 Time evolution of vertical velocity at $5 \mathrm{~km}$ and tangential wind at $1 \mathrm{~km}$ from the control run, annulus, doubling RMW, and large PV skirt experiments. 
(a) time $=40 \mathrm{~h}$

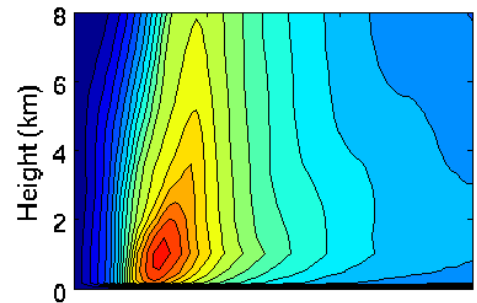

(d) time $=52 \mathrm{~h}$

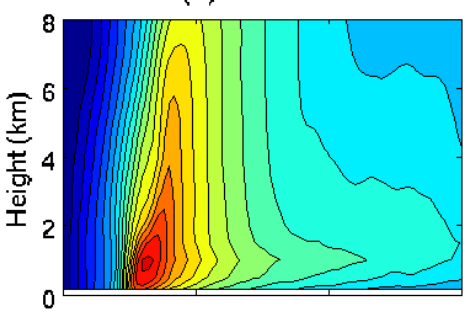

(g) time $=64 \mathrm{~h}$

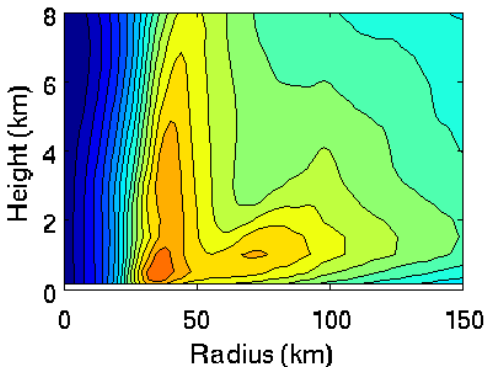

(b) time $=44 \mathrm{~h}$

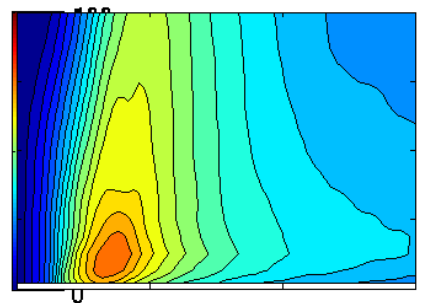

(e) time $=56 \mathrm{~h}$

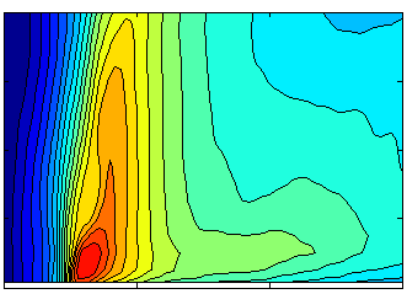

(h) time $=68 \mathrm{~h}$

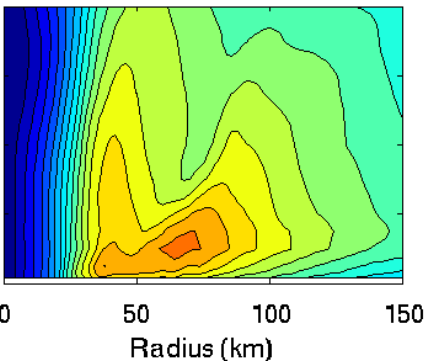

(c) time $=48 \mathrm{~h}$

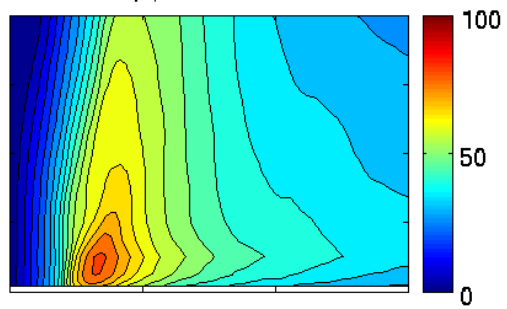

(f) time $=60 \mathrm{~h}$

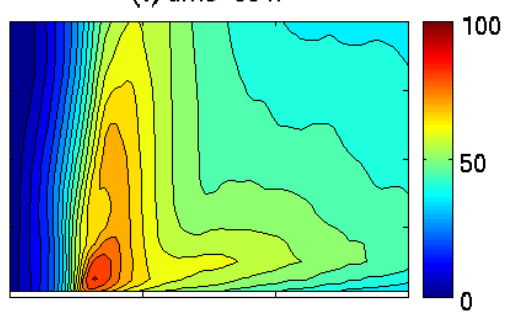

(i) time $=72 \mathrm{~h}$

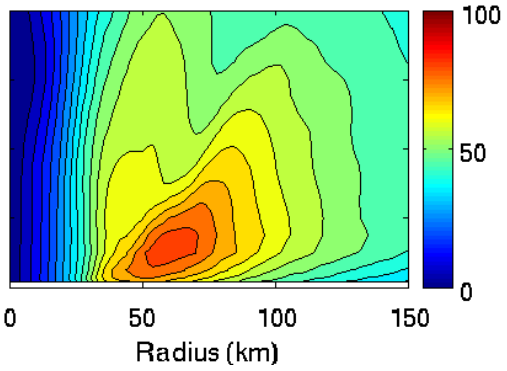

Figure 4.20 Height-radius plots of tangential wind at different times during the ERC period from the double RMW experiments. 
(a) time $=40 \mathrm{~h}$

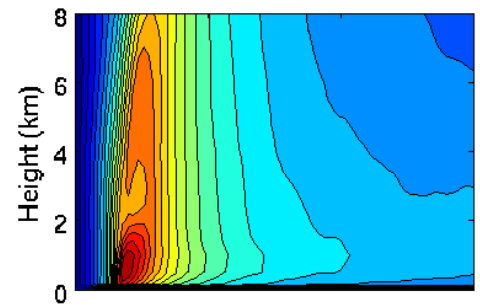

(d) time $=52 \mathrm{~h}$

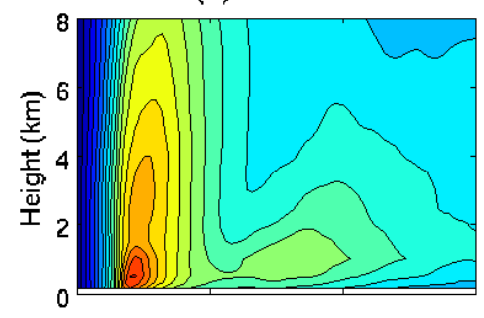

(g) time $=64 \mathrm{~h}$

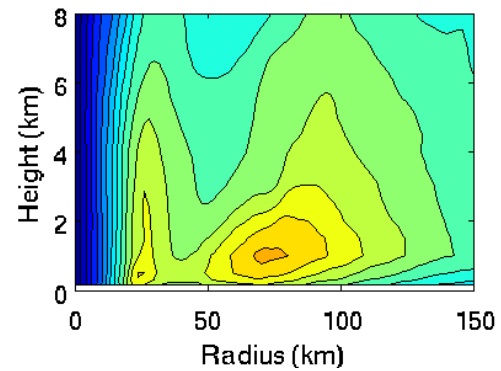

(b) time $=44 \mathrm{~h}$

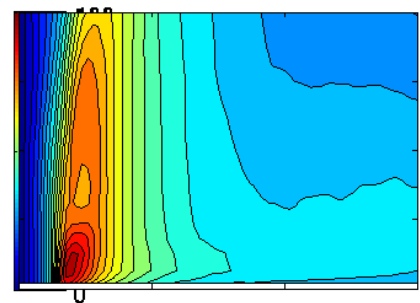

(e) time $=56 \mathrm{~h}$

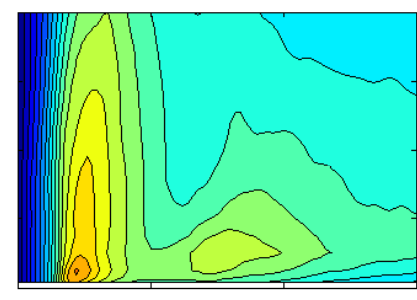

(h) time $=68 \mathrm{~h}$

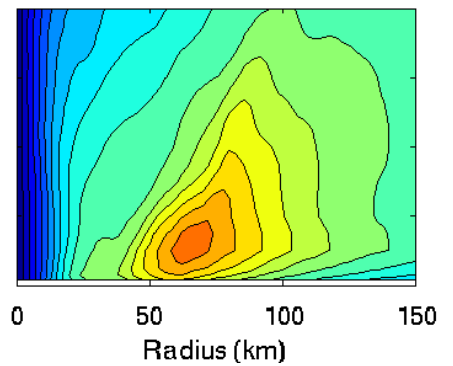

(c) time $=48 \mathrm{~h}$

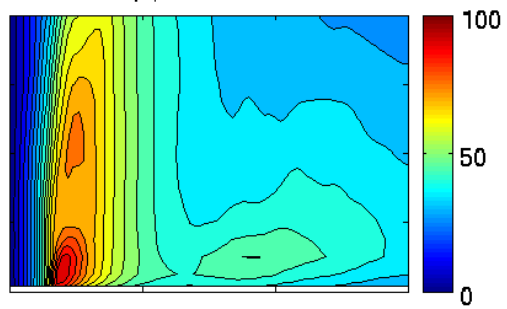

(f) time $=60 \mathrm{~h}$

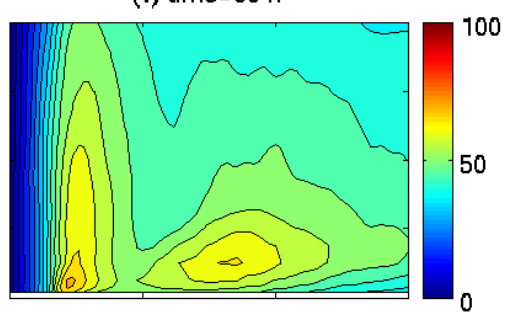

(i) time $=72 \mathrm{~h}$

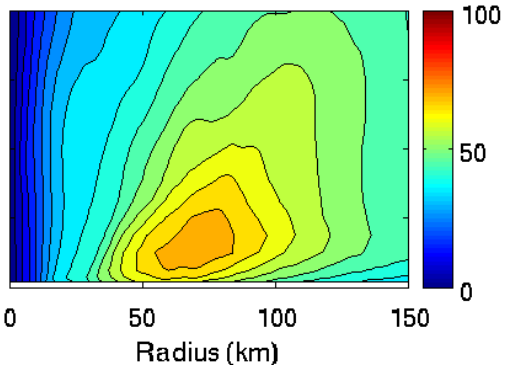

Figure 4.21 Height-radius plots of tangential wind at different times during the ERC period from the large PV skirt experiments. 
(a) $\mathrm{w}$ at $5 \mathrm{~km}$ (Control run)

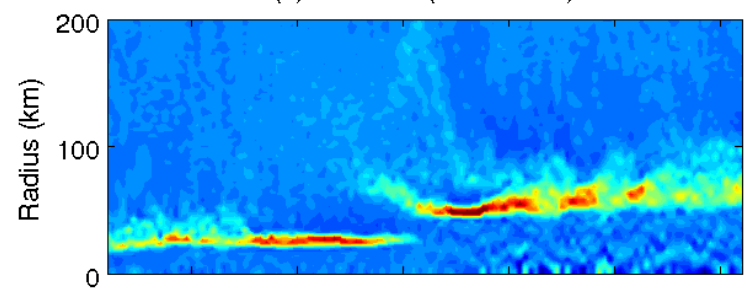

(c) $\mathrm{w}$ at $5 \mathrm{~km}$ (double snow terminal velocity)

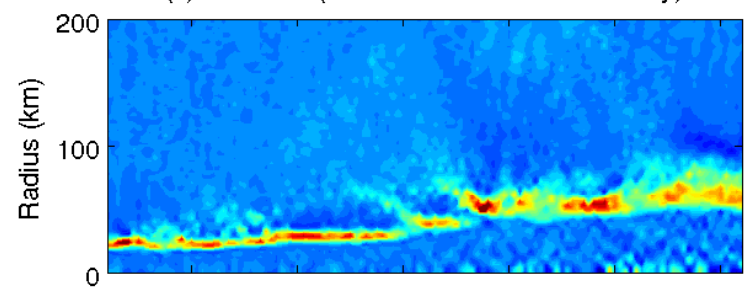

(e) $w$ at $5 \mathrm{~km}$ (half snow terminal velocity)

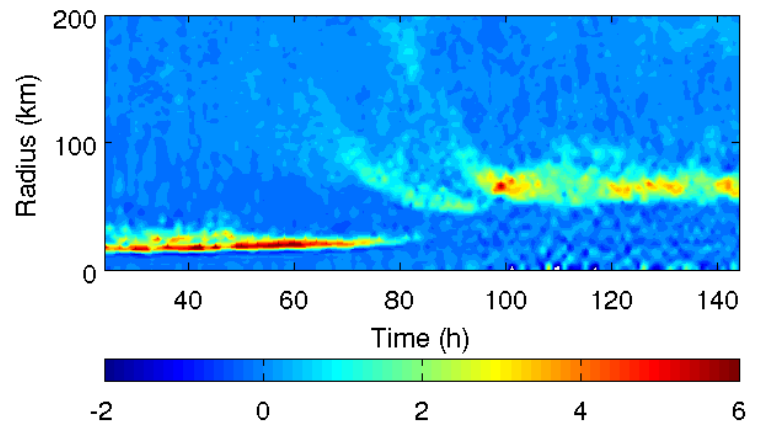

(b) Vt at $1 \mathrm{~km}$ (Control run)

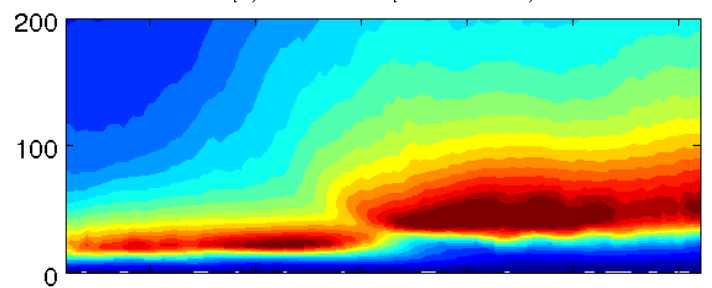

(d) Vt at $1 \mathrm{~km}$ (double snow terminal velocity)

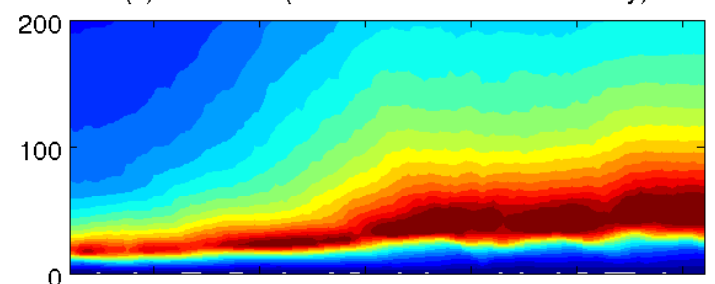

(f) $\mathrm{Vt}$ at $1 \mathrm{~km}$ (half snow terminal velocity)

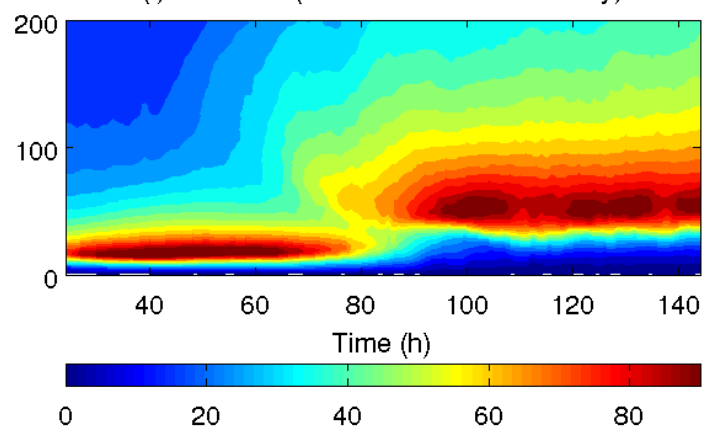

Figure 4.22 Time evolution of vertical velocity at $5 \mathrm{~km}$ and tangential wind at $1 \mathrm{~km}$ from the control run and large/small snow terminal velocity experiments. 
(a) Control run

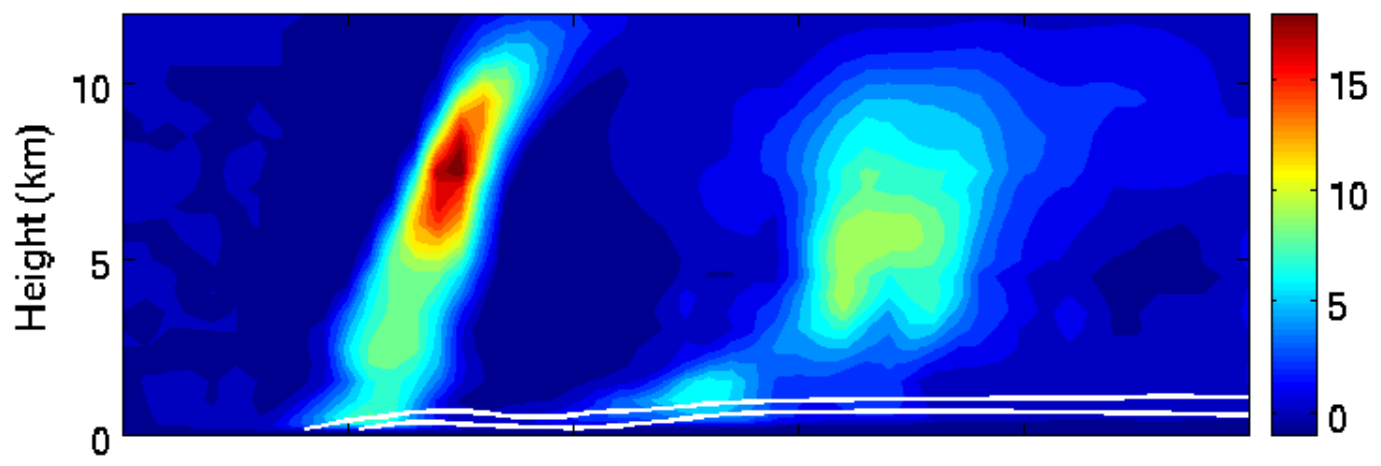

(b) Doubling snow terminal velocity

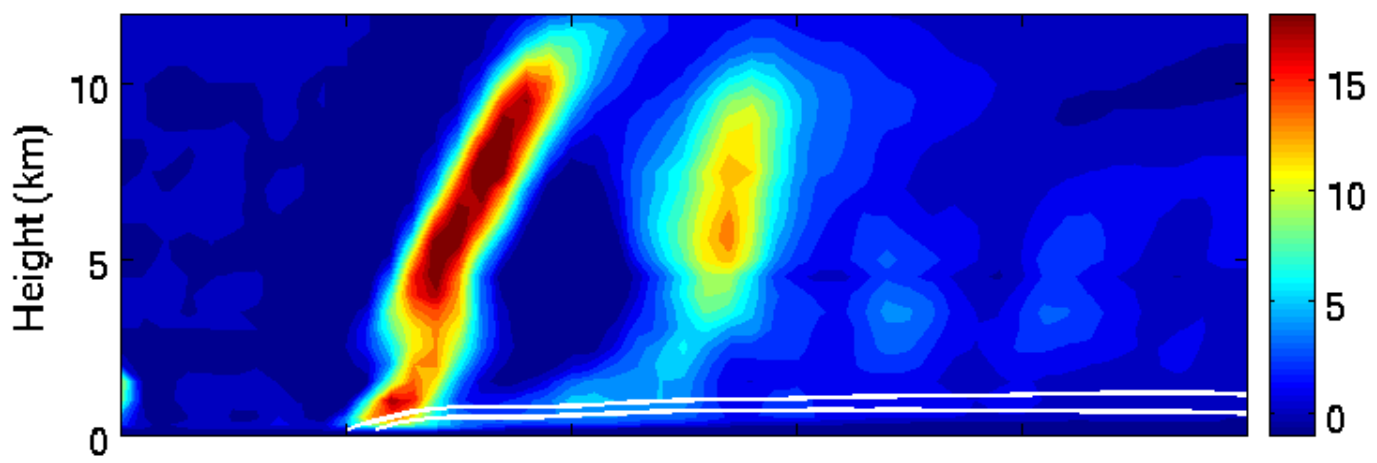

(c) Reducing snow terminal velocity bu half

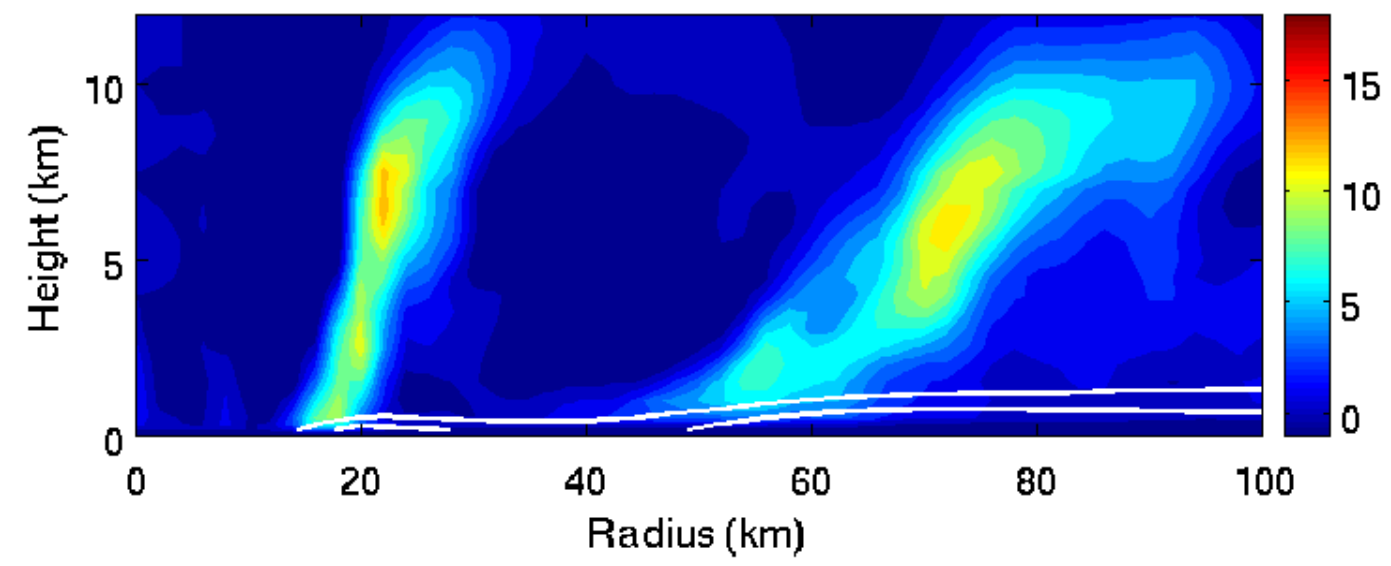

Figure 4.23 Diabatic heating (color shades) and radial inflow (white contours, $-3 \mathrm{~m} / \mathrm{s}$ and $-12 \mathrm{~m} / \mathrm{s}$ ) at the $78^{\text {th }} \mathrm{h}$ from the control run and large/small snow terminal velocity experiments. 
(a) $w$ at $5 \mathrm{~km}$ (Cont)

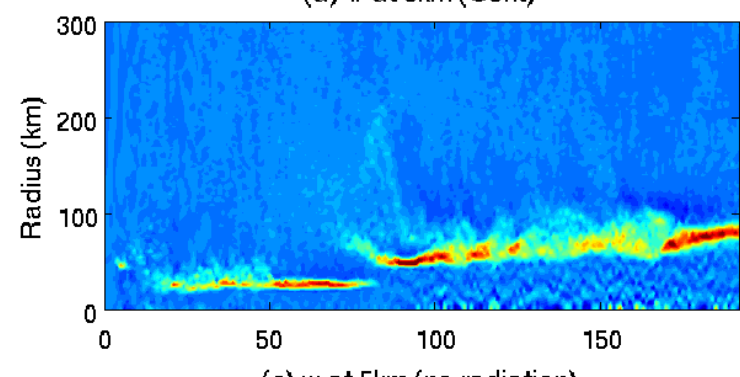

(c) $w$ at $5 \mathrm{~km}$ (no radiation)

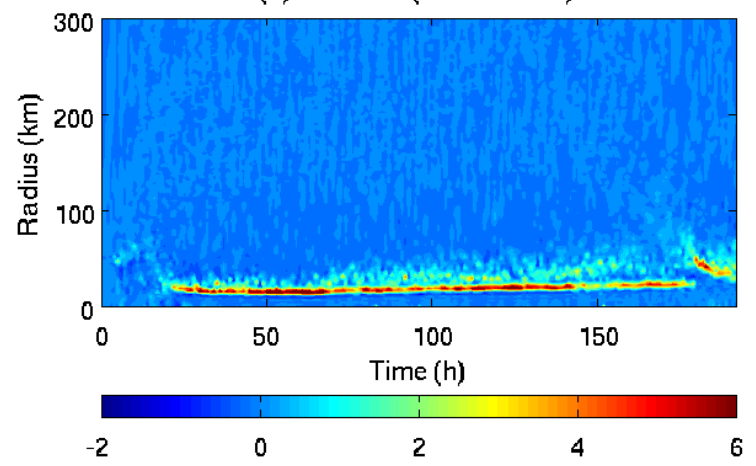

(b) Vt at $1 \mathrm{~km}$ (Cont)

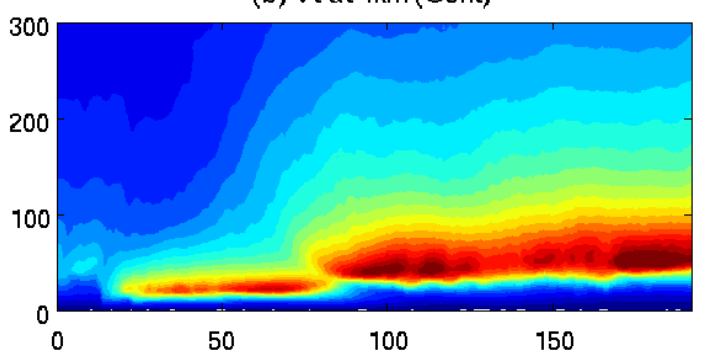

(d) $V t$ at $1 \mathrm{~km}$ (no radiation)

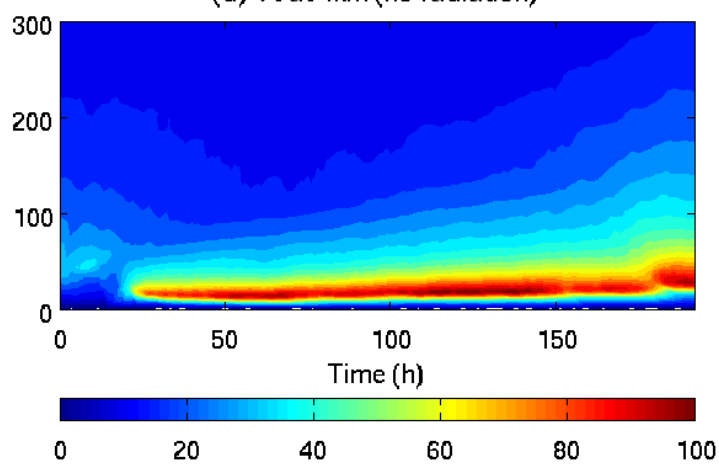

Figure 4.24 Time evolution of vertical velocity at $5 \mathrm{~km}$ and tangential wind at $1 \mathrm{~km}$ from the control run and the no radiation experiment. 
(a) $w$ at $5 \mathrm{~km}$ (Control run)

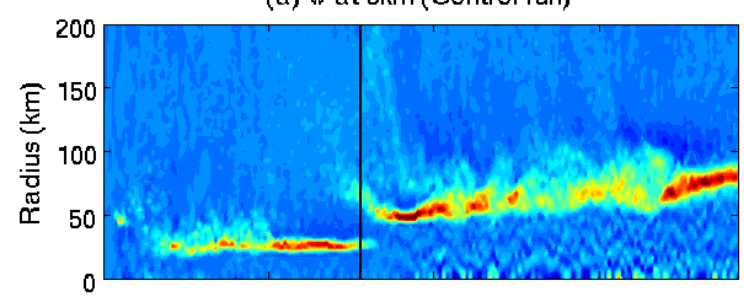

(c) $w$ at $5 \mathrm{~km}(5 \mathrm{~m} / \mathrm{s}$ background wind)

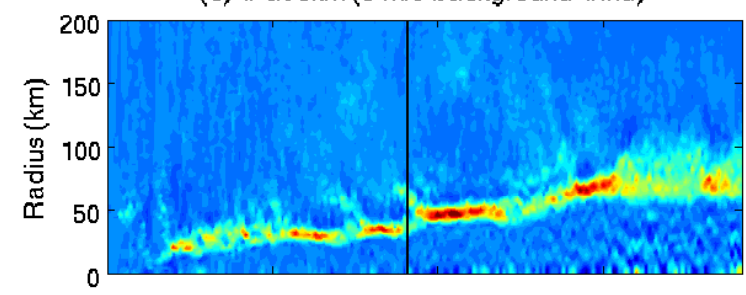

(e) $w$ at $5 \mathrm{~km}(10 \mathrm{~m} / \mathrm{s}$ background wind)

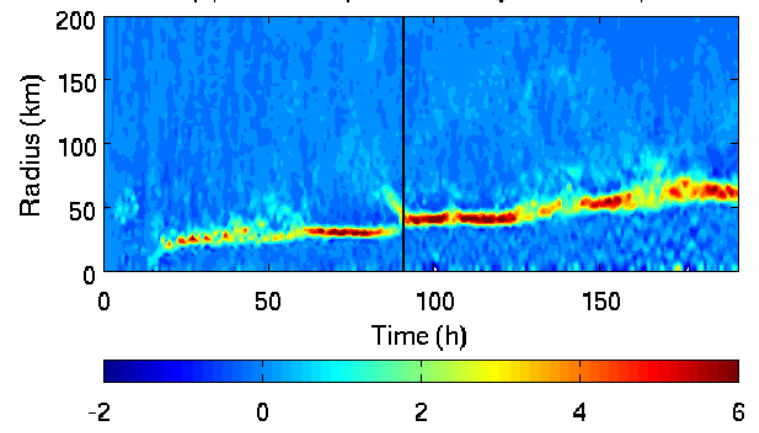

(b) Vt at $1 \mathrm{~km}$ (Control run)

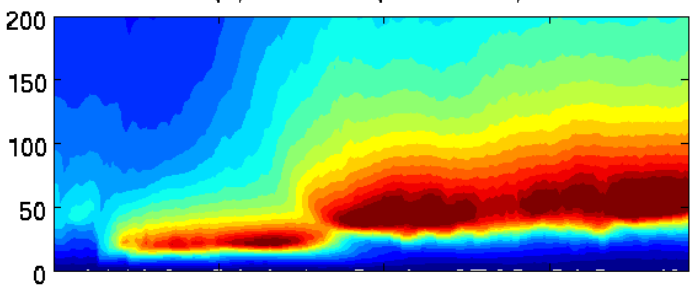

(d) Vt at $1 \mathrm{~km}$ ( $5 \mathrm{~m} / \mathrm{s}$ background wind)

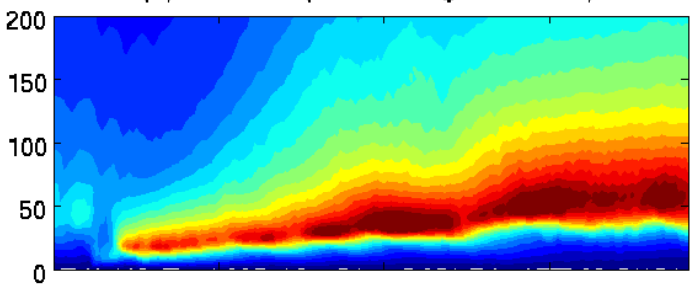

(f) $\mathrm{Vt}$ at $1 \mathrm{~km}$ ( $10 \mathrm{~m} / \mathrm{s}$ background wind)

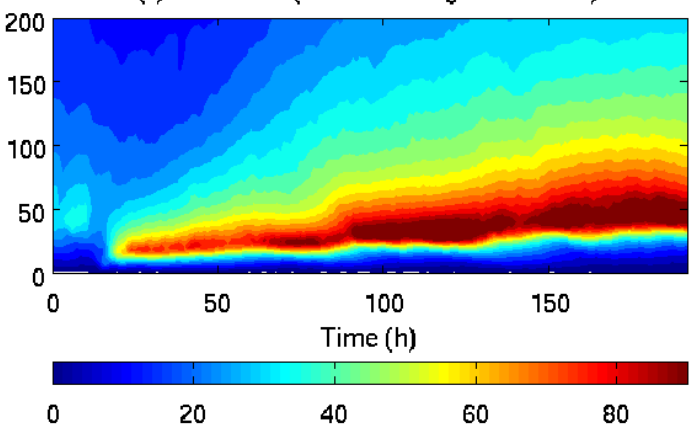

Figure 4.25 Time evolution of vertical velocity at $5 \mathrm{~km}$ and tangential wind at $1 \mathrm{~km}$ from the control run and sensitivity experiments with background wind of 5 $\mathrm{m} / \mathrm{s}$ and $10 \mathrm{~m} / \mathrm{s}$, respectively. The vertical black line in (a), (c), and (e) indicates the time for the height-radius structure analyses shown in Figure 4.26 . 
(a) Diabatic heating at $78 \mathrm{~h}$ (Control run)

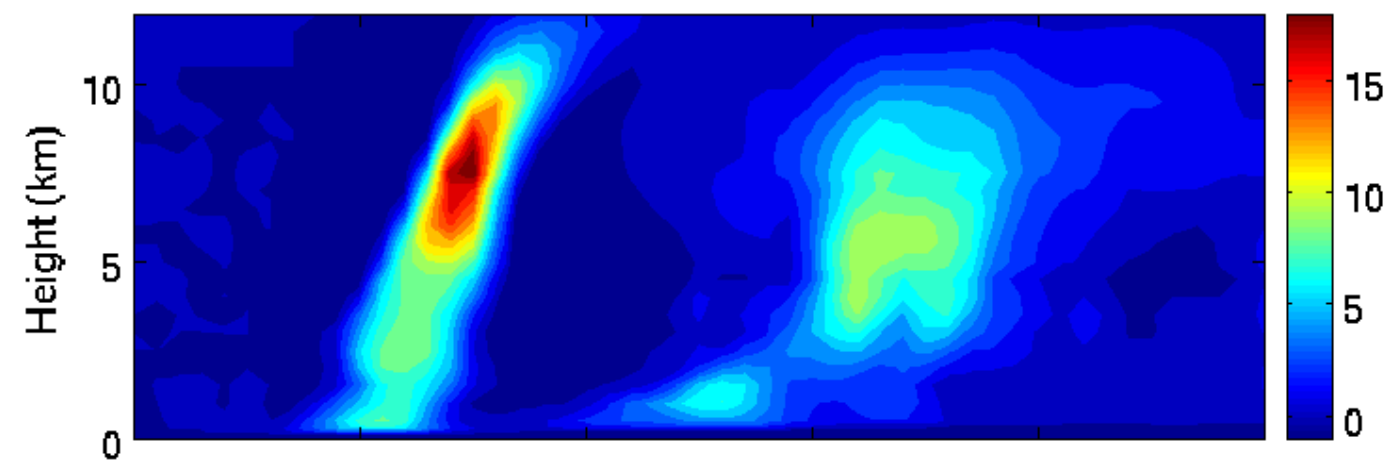

(b) Diabatic heating at $91 \mathrm{~h}$ (BGW05)

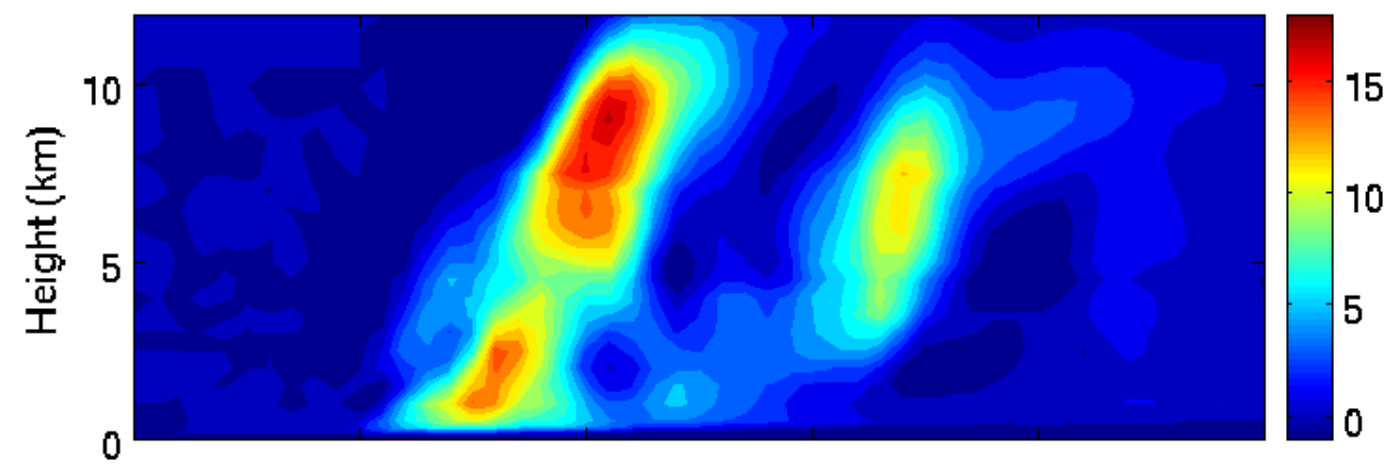

(c) Diabatic heating at $91 \mathrm{~h}(\mathrm{BGW} 10)$

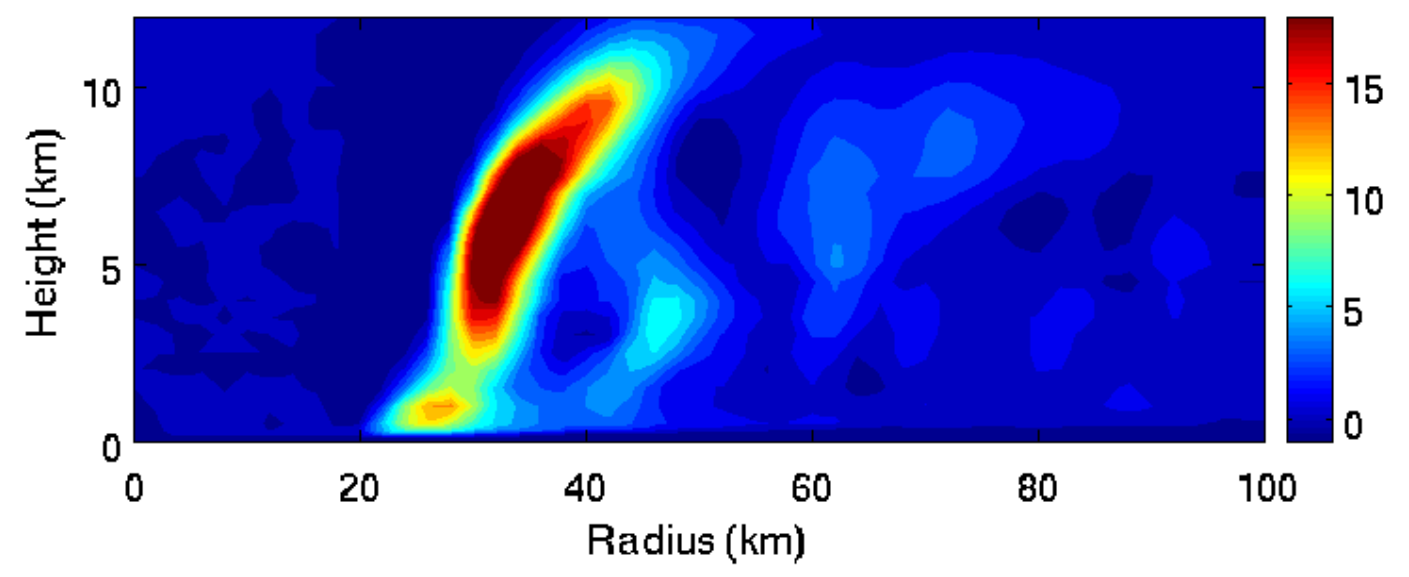

Figure 4.26 Height-radius plots of diabatic heating at an arbitrary time (indicated in Figure 4.25) during the ERC period from the control run (a), experiments with background wind of $5 \mathrm{~m} / \mathrm{s}$ (b) and $10 \mathrm{~m} / \mathrm{s}(\mathrm{c})$. 
(a) Large skirt without bgw

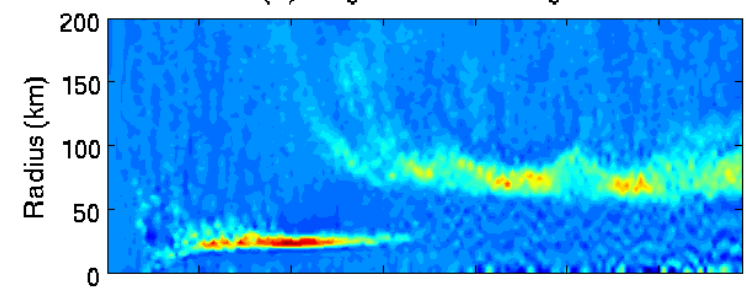

(c) Large skirt with bgw $=5 \mathrm{~m} / \mathrm{s}$

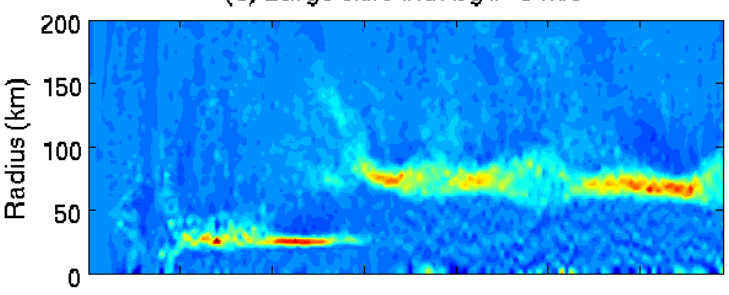

(e) Large skirt with bgw $=10 \mathrm{~m} / \mathrm{s}$

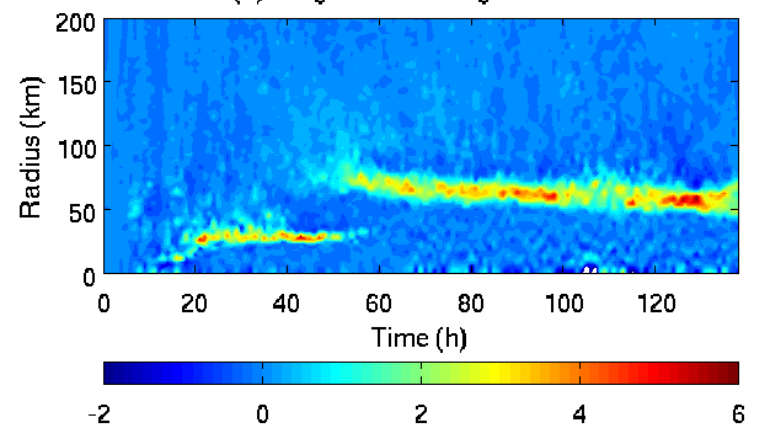

(b) Large skirt without bgw

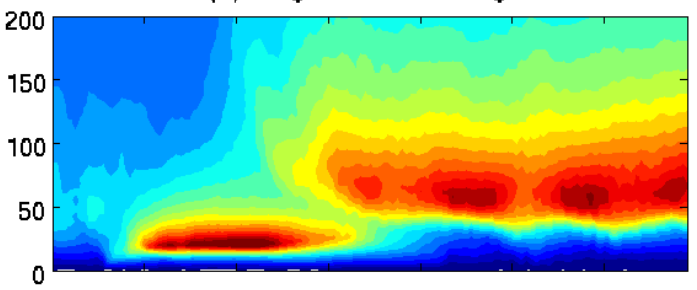

(d) Large skirt with $\mathrm{bgw}=5 \mathrm{~m} / \mathrm{s}$

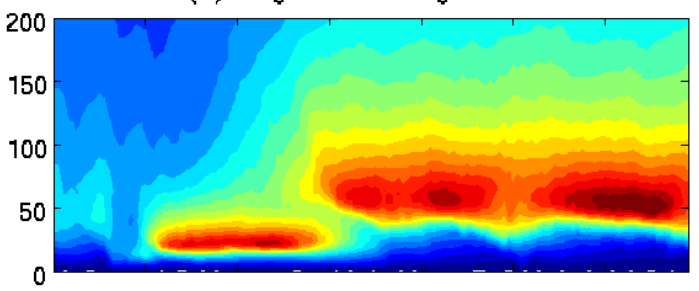

(f) Large skirt with bgw $=10 \mathrm{~m} / \mathrm{s}$

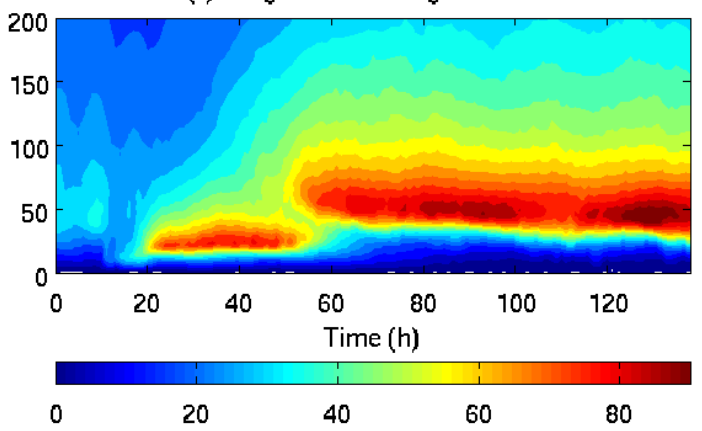

Figure 4.27 Time evolution of vertical velocity at $5 \mathrm{~km}$ and tangential wind at $1 \mathrm{~km}$ from the three sensitivity experiments on the initial vortex with a large PV skirt, (a) without background wind, (b) with $5 \mathrm{~m} / \mathrm{s}$ prescribed background wind, and (c) with $10 \mathrm{~m} / \mathrm{s}$ prescribed background wind. 


\section{Chapter 5 Conclusions and future work}

Eyewall replacement cycle (ERC), which is often accompanied with dramatic changes in intensity and inner-core structure of a tropical cyclone (TC), is frequently observed during the evolution of an intensifying TC. Although intensely studied in recent years, the underlying mechanisms that goven ERC are still poorly understood, and a correct timely forecasting of ERC remains to be a great challenge in numerical weather prediction. To advance our understanding of this unique phenomenon of TCs and provide insights into the improvement of numerical forecast of ERC, in this dissertation study, a series of carefully designed $3 \mathrm{~d}$ full physics simulations by weather research \& forecasting (WRF) model is performed to investigate ERC in TC-like vortices constructed from idealized wind and vorticity profiles on a f-plane. Using these simulations, this dissertation addresses three critical but yet unanswered questions of ERC: (1) what is the role of outer rainband convection in ERC including the secondary eyewall formation (SEF) and the demise of inner primary eyewall? (2) what physical processes determine the formation and development of secondary maximum of tangential wind? and (3) how SGS parameterizations, TC vortex radial structure, and background wind affect SEF and ERC in 3D full physics numerical simulations? In this study, we attempted to address all these three questions in a balanced symmetric framework because to the first order approximation ERC may be considered as an axisymmetric phenomenon although it involves both $3 \mathrm{~d}$ symmetric and asymmetric processes, and some of them are not balanced particularly for those in the boundary layer. However, from the evidence provided throughout this dissertation, which will be summarized shortly, the asymmetric unbalanced processes do not 
appear to have a substantial effect to alter the main conclusions of this study. The major tool that we used to analyze the simulated data for addressing these three questions is the Sawyer-Eliassen equation, which was first introduced by SW82 for understanding the evolution of balanced symmetric TC-like vortices via the transverse circulation induced by point heat and momentum sources. Here we show that this powerful diagnostic tool provides an appropriate means to analyze physical processes governing ERC in $3 \mathrm{~d}$ full physics simulations that can be well understood in the balanced symmetric framework. The main conclusions obtained from our simulations and diagnoses are summarized as follows:

1. Although our simulations are started from an idealized vortex constructed from the prescribed profiles of tangential wind and vorticity, the simulated ERC in the control run captures all the key features of observed ERC in real TCs summarized from various observations. These include (a) an apparent secondary wind maximum associated with the outer concentric eyewall; (b) weakening in storm intensity as the concentric outer ring of convection forms; (c) reintensification of the storm after ERC completes; and (d) a larger radius of maximum wind (RMW) than that before ERC occurring. This gives us confidence that the simulations performed in this study are appropriate and can be used to address various issues of ERC.

2. Detailed comparisons between the vertical velocities and radial flow directly simulated by WRF and those diagnosed by the Sawyer-Eliassen equation show that there is a very good agreement between them before, during, and after ERC. The correlation coefficients between the vertical velocity and radial inflow simulated by WRF and the 
Sawyer-Eliassen diagnoses during the ERC period exceeds 0.8 for most areas away from the lateral boundaries below $16 \mathrm{~km}$ and above the boundary layer. Even in the boundary layer, the correlation coefficients are still greater than 0.6, suggesting that the SawyerEliassen equation provides a useful analytical tool for investigating SEF and ERC in realistic 3D full physics simulations.

3. The tangential wind budget analyses reveal several important facts of the formation and development of concentric rings of tangential wind during the ERC period. First, the net tendency of tangential wind is a small residual resulted from the cancellation between two large tendencies induced by the model resolved processes and the sub-grid scale (SGS) processes. Second, the resolved processes are responsible for generating positive tendencies of tangential wind in the boundary layer, whereas the SGS tendency is negative. Since ERC involves the development of a secondary wind maximum, which requires a positive tendency, it suggests that the resolved processes are the driving force for ERC and the SGS processes may be considered as a response to the changes induced by the resolved processes. However, since the SGS tendency has a magnitude comparable to that of resolved tendency, the SGS parameterizations can have a profound impact on the occurrence and characteristics of ERC, posing a great challenge for numerical forecasting of ERC. Third, the tendency induced by the SGS vertical mixing is nearly 20 times larger than that induced by the SGS horizontal mixing. This result suggests that SGS horizontal mixing only plays a minor role in ERC although recent numerical studies show that it can have a substantial impact on TC development (e.g., Bryan and Rotunno et al. 2009; Rotunno et al. 2009). Fourth, compared with leading terms in the budget, the tendency induced by the resolved asymmetric eddies is 
small and generally negative in the boundary layer, indicating that asymmetric eddies are not a driving force for the development of the secondary wind maximum. Finally, the resolved horizontal advection of absolute vorticity and vertical advection of tangential wind are the two leading contributions in the budget but they have opposite signs within and above the boundary layer. The cancellation of the two terms, however, results in the positive tendency of tangential wind in the boundary layer, which is responsible for the development of secondary maximum of tangential wind.

4. With the Sawyer-Eliassen diagnoses, we are able to answer Question 1 and 2 raised at the beginning of the dissertation. The separated contribution from eyewall and outer rainband convection by the Sawyer-Eliassen equation clearly indicates that the eyewall forcing alone can never generate a secondary wind maximum. It is the outer rainband diabatic heating that induces a large tangential wind tendency leading to the formation of secondary wind maximum. The Sawyer-Elisssen diagnoses further showed that the outer rainband convection must reach a certain strength relatively to the eyewall convection, approximately $10 \%$ according to this study, before it can initiate and drive the secondary wind maximum via horizontal advection of absolute vorticity and vertical advection of tangential wind. In practice, the ratio of strength of outer rainband convection to that of eyewall convection may be used as a predictor for a timely forecast of ERC.

5. Our analyses reveal a positive feedback among low-level convection, convergence of radial inflow, acceleration of tangential wind in the upper boundary layer, and surface evaporation that leads to the development of outer rainband convection and the subsequent ERC, which may be described as follows: The sporadic convection in the 
outer rainband region induces the convergence of radial inflow and accelerates the tangential wind at the base of convection, which increases the moisture in the low troposphere, and thus, favors the development of low level convection, which, according to the Sawyer-SEliassen diagnoses, can result in convergence of radial inflow and acceleration of tangential wind in the upper boundary layer. While the former leads to the effective transport of moisture into the outer rainband convection, the latter enhances the surface evaporation. Both processes can foster further development of convection. This can cause a runaway interaction leading to the development of outer rainband convection and the formation of secondary wind maximum.

6. It is found that the eyewall heating can induce fairly strong subsidence over the outer rainband region and the outer rainband heating can induce non-negligible subsidence over the eyewall updraft, which is different from the previous study by Rozoff et al. (2008) who showed that the eyewall and outer rainband heating is mainly concentrated in the eye and moat based on an idealized TC model. However, the demise of inner eyewall to end an ERC appears to be intimately involved with the development of radial flow. The decomposed radial flow by the Saywer-Eliassen equation clearly indicates that the radial outflow induced by the outer rainband convection provides a mechanism to cancel the radial inflow induced by the eyewall. When the outer rainabnd convection becomes sufficiently strong, its induced radial outflow can extend throughout the vertical column from the lower boundary layer to tropopause, which works as a barrier to cut off the radial inflow into the inner eyewall. This cutting off fuel into the inner eyewall by the outer rainband induced outflow causes the inner eyewall to decay rapidly and disappear quickly. 
7. The SGS vertical turbulent mixing scheme can substantially affect the characteristics of simulated ERC, in particular, the concentric ring structure of tangential wind. Such an impact of SGS vertical turbulent mixing on ERC is through a complicated interaction among eyewall/outer rainband heating, radila inflow in the boundary layer, surface layer processes, and shallow convection in the moat. Our sensitivity experiments show that different SGS vertical turbulent mixing schemes can lead to different vertical structures of eyewall diabatic heating, which, according to the Sawyer-Eliassen diagnoses, can result in radial inflow with different strength. For the eyewall diabatic heating with its maximum located approximately at the freezing level, stronger radial inflow can be generated far beyond the moat region. The moisture transported into the moat provides an important energy source for the development of shallow convection in the moat, which accelerates the surface wind to enhance surface evaporation here, and hence, further promoting shallow convection. The enhanced shallow convection in the moat allows the outer rainband convection to move closer to the eyewall, leaving little room for the outer rainband convection to develop a clean secondary maximum of tangential wind. In contrast, the eyewall diabatic heating with its maximum located above the freezing level generates a weaker radial inflow, which can barely extend radially beyond the moat. Without sufficient energy supply, the shallow convection in the moat is suppressed by the subsidence induced by the eyewall and outer rainband heating. As a result, the outer rainband heating can be cleanly separated from the eyewall heating to generate well-defined concentric rings of tangential wind. Our simulations also show that different SGS vertical turbulent mixing scheme can yield different radial temperature profiles in the surface layer. For example, the YSU scheme generates a profile with the minimum temperature oc- 
curring in the moat in contrast to the one generated by the MYJ and MYYNN schemes that gradually decreases radially outward. The different temperature profile in the surface layer can lead to different convective instability to further affect the shallow convection in the moat assuming that SST is constant. The sensitivity experiments show that the different characteristics of ERC resulted from different SGS vertical turbulent mixing schemes to some extent can be reproduced by a scheme through adjusting the mixing length used in the scheme. Since the mixing length largely determines the eddy viscosity, this result suggests the importance of eddy viscosity to the formation and development of concentric ring structure of tangential wind during an ERC.

8. Snow terminal velocity poses a great impact on the concentric ring structure of tangential wind associated with an ERC. Large snow terminal velocity causes snow to fall rapidly and melt in the low level, and therefore, enhances the diabatic heating to result in a strong radial inflow in the boundary layer, which favors the development of shallow convection in the moat allowing the outer rainband convection to move closer to the eyewall, leaving little room for a full development of a secondary maximum of tangential wind for the reason that has been stated in conclusion 7. On the other hand, small snow terminal velocity may not be able to generate a radial inflow sufficiently strong to foster shallow convection in the moat, so that clean concentric rings of tangential winds may develop by the separated eyewall and outer rainband heating.

9. The occurrence of ERC shows a substantial sensitivity to radiation. The sensitivity tests show that the outer rainband activity in the no-radiation experiment is exceptionally quiet and it takes a long time for the outer rainband convection to develop, which 
eventually leads to an ERC event. We argue that the inactive shallow convection due to lack of cloud-top radiative cooling provides an unfavorable condition to promote deep convection in the outer rainband region.

The simulations performed in this study also raise a few unanswered questions regarding ERC that need to be further investigated in future research:

1. The detailed procedure of outer rainband convective activity governs the evolution of an ERC in numerical simulations has been clearly demonstrated and described from a symmetric perspective in this study. One aspect of this argument that has not been thoroughly addressed is the radially inward propagation of outer rainbands. Although we attempted to provide an explanation in the same symmetric framework that we used to address ERC, propagation of outer rainband is fundamentally an asymmetric problem. Moreover, as shown by the sensitivity experiments, the development and propagation of outer rainbands are sensitive to model physics, such as SGS vertical turbulent mixing scheme, microphysical scheme, and radiative transfer calculation. To clarify all these issues of outer rainband activity and propagation, further investigations are needed.

2. The simulations show that ERC, particularly the concentric ring structure of tangential wind, is sensitive to the vortex radial structure. It is found that large RMW and PV skirt tend to yield clean concentric rings of tangential wind with a long duration, whereas small RMW and PV skirt may end up with an ERC without a secondary maximum tangential wind or may generate double-eyewall replacement. However, the mechanism underlying this sensitivity is not understood and it is currently unknown if these features of ERC and their relationship to vortex radial structure shown in simulations are 
also seen in observations. These questions cannot be answered solely by the idealized simulations used in this study. To clarify these issues, long term observation data and simulations of real TCs with different vortex structures are needed, which will be one of the focuses of our future study.

3. In this study, we have demonstrated the sensitivity of ERC to snow terminal velocity. However, snow terminal velocity is just one of many important parameters used in a microphysical scheme. It remains unclear how other parameters affect ERC. More sensitivity experiments and in-depth analyses are needed to thoroughly understand the impact of microphysics on ERC.

4. Our sensitivity experiments show that depending on the structure of a TC vortex the background wind may substantially change the characteristics of an ERC. For example, a sufficiently large background wind may completely smooth out an ERC, which would otherwise occur without background wind, but it appears to have little impact on an ERC if the TC vortex has a sufficiently large PV skirt. Although the impact of background wind on ERC and its dependence on TC vortex structure are clearly shown in the sensitivity experiments, the underlying mechanisms have not been explored. Analyzing these sensitivity experiments and obtaining a better understanding of the role of background wind in ERC will be the immediate goal of our future research. 


\section{References}

Abarca, S. F., and M. T. Montgomery, 2013: Essential Dynamics of Secondary Eyewall Formation. J. Atmos. Soc., 70, 3216-3230

Adams, J. (1989), MUDPACK: Multigrid Fortran Software for the Efficient Solution of Linear Elliptic Partial Differential Equations, Applied Math. and Comput. 34, 113-146, doi: http://dx.doi.org/10.1016/0096-3003(89)90010-6

Black, R. A., and J. Hallett, 1986: Observations of the distribution of ice in hurricanes. $J$. Atmos. Sci., 43, 802-822.

Braun, S. A., and W.-K. Tao, 2000: Sensitivity of high-resolution simulations of Hurricane Bob (1991) to planetary boundary layer parameterizations. Mon. Wea. Rev., 128, 3941-3961.

Bryan, G. H, and R. Rotunno, 2009: The maximum intensity of tropical cyclones in axisymmetric numerical model simulations. Mon. Wea. Rev., 137, 1770-1789.

Bui, H. H., R. K. Smith, M. T. Montgomery, and J. Peng, 2009: Balanced and unbalanced aspects of tropical cyclone intensification. Quart. J. Roy. Meteor. Soc., 135, 1715-1731.

Charnok, H., 1955: Wind stress on a water surface. Quart. J. Roy. Meteor. Soc., 81, 639640.

Chen S. S., J. Knaff, and F. D. Marks, 2006: Effects of vertical wind shear and storm motion on tropical cyclone rainfall asymmetries deduced from TRMM. Mon. Wea. Rev., 134, 3190-3208.

Davis C., and Coauthors, 2008: Prediction of landfalling hurricanes with the Advanced Hurricane WRF model. Mon. Wea. Rev., 136, 1990-2005.

Donelan,M. A., B. K. Haus, N. Reul, W. J. Plant, M. Stiassne, H. C. Graber, O. B. Brown, and E. S. Saltzman, 2004: On the limiting aerodynamic roughness of the ocean in very strong winds. Geophys. Res. Lett., 31, L18306, doi:10.1029/2004GL019460.

Dudhia, J., 1989: Numerical study of convection observed during the winter monsoon experiment using a mesoscale two-dimensional model. J. Atmos. Sci., 46, 3077-3107.

Dunion, J. P., C. S. Marron, 2008: A reexamination of the Jordan mean tropical sounding based on awareness of the Saharan air layer: Results from 2002. J. Climate., 21, 52425253.

Eliassen, A., 1951: Slow thermally or frictionally controlled meridional circulation in a circular vortex. Astrophys. Norv., 5, 19-60.

Emanuel, K. A., 1986: An air-sea interaction theory for tropical cyclone. Part I: Steady state maintenance. J. Atmos. Sci., 43, 585-604. 
Fang, J., and F. Zhang, 2012: Effect of Beta Shear on Simulated Tropical Cyclones. . Mon. Wea. Rev., 140, 3327-3346.

Fierro A. O., R. F. Rogers, F. D. Marks, and D. S. Nolan, 2009: The impact of horizontal grid spacing on the microphysical and kinematic structures of strong tropical cyclones simulated with the WRF-ARW model. Mon. Wea. Rev., 137, 3717-3743.

Fovell, R. G., K. L., Corbosiero, and H. C. Kuo, 2009: Cloud microphysics impact on hurricane track as revealed in idealized experiments. J. Atmos. Sci., 66, 1764-1778.

Fudeyasu, H., and Y. Wang, 2011: Balanced contribution to the intensification of a tropical cyclone simulated in TCM4: Outer-core spinup process. J. Atmos. Sci., 68, 430-449.

Hawkins, H. F., 1983: Hurricane Allen and island obstacles. J. Atmos. Sci.,40, 1360-1361.

Hawkins, J. D., and M. Helveston, 2008: Tropical cyclone multiple eyewall characteristics. Preprints, 28th Conf. on Hurricanes and Tropical Meteorology. Orlando, FL, Amer. Meteor.Soc., 14B.1.

Hill, K. A., and G. M. Lackmann, 2009a: Influence of environmental humidity on tropical cyclone size. Mon. Wea. Rev., 137, 3294-3315.

Hill, K. A., and G. M. Lackmann, 2009b: Analysis of idealized tropical cyclone simulations using the Weather Research and Forecasting model: Sensitivity to turbulence parameterization and grid spacing. Mon. Wea. Rev., 137, 745-765.

Hong S. Y., Y. Noh, and J. Dudhia, 2006: A new vertical diffusion package with an explicit treatment of entrainment processes. Mon. Wea. Rev., 134, 2318-2341.

Hong, S. Y., J. Dudhia, and S. H. Chen, 2004: A revised approach to ice microphysical processes for the bulk parameterization of clouds and precipitation. Mon. Wea.

Rev., 132, 103-120.

Houze, R. A., S. S. Chen, B. F. Smull, W.-C. Lee, and M. M. Bell, 2007: Hurricane intensity and eyewall replacement. Science, 315, 1235-1239.

Huang, Y.-H., M. T. Montgomery, and C.-C. Wu, 2012: Concentric eyewall formation in Typhoon Sinlaku (2008). Part II: Axisymmetric dynamical processes. J. Atmos. Soc., 69, $662-674$.

Irish, J. L., D. T. Resio, and J. J. Ratcliffe, 2008: The influence of storm size on hurricane surge. J. Phys. Oceanogr., 38, 2003-2013.

Janjic, Z. I., 1996: The surface layer in the NCEP Eta Model. Preprints, 11th Conf. on Numerical Weather Prediction, Norfolk, VA, Amer. Meteor. Soc., 354-355.

Janjic, Z. I., 2002: Nonsingular implementation of the Mellor-Yamada level 2.5 scheme in the NCEP Meso model. NCEP Office Note 437, 61 pp. 
Jordan, C. L., 1958: Mean soundings for the West Indies area. J. Meteor., 15, 91-97.

Judt, K., and S. S. Chen, 2010: Convectively generated potential vorticity in rainbands and formation of the secondary eyewall in Hurricane Rita of 2005. J. Atmos. Sci., 67, 3581-3599.

Kain, J. S., 2004: The Kain-Fritsch convective parameterization: an update.Journal of Applied Meteorology, 43, 170-181.

Kaplan, J., and M. DeMaria, 2003: Large-scale characteristics of rapidly intensifying tropical cyclones in the North Atlantic basin. Wea. Forecasting, 18, 1093-1108.

Kepert, J. D., 2012: Choosing a boundary layer parameterization for tropical cyclone modeling. Mon. Wea. Rev., 140, 1427-1445.

Kepert, J. D., 2013: How Does the Boundary Layer Contribute to Eyewall Replacement Cycles in Axisymmetric Tropical Cyclones?. J. Atmos. Soc.,70, 2808-2830

Kuo, H.-C., L.-Y. Lin, C.-P. Chang, and R. T. Williams, 2004: The formation of concentric vorticity structures in typhoons. J. Atmos. Sci., 61, 2722-2734.

Kuo, H.-C., W. H. Schubert, C.-L. Tsai, and Y.-F. Kuo, 2008: Vortex interactions and barotropic aspects of concentric eyewall formation. Mon. Wea. Rev., 136, 5183-5198.

Kuo, H-C., R. T. Williams, and J-H. Chen, 1999: A possible mechanism for the eye rotation of Typhoon Herb. J. Atmos. Sci, 56, 1659-1673.

Landsea, C. W., J. L. Franklin, C. J. McAdie, J. L. Beven, J. M. Gross, B. R. Jarvinen, R. J. Pasch, E. N. Rappaport, J. P. Dunion, and P. P. Dodge, 2004: A reanalysis of Hurricane Andrew's intensity. Bull. Am. Meteorol. Soc., 85, 1699-1712.

Laprise, R., 1992: The Euler equations of motion with hydrostatic pressure as an independent variable. Mon. Wea Rev., 120, 197-207.

$\mathrm{Li}, \mathrm{X}$. and Z. Pu, 2008: Sensitivity of numerical simulation of early rapid intensification of Hurricane Emily (2005) to cloud microphysical and planetary boundary layer parameterizations . Mon. Wea. Rev.136, 4819-4838,

Maclay, K. S., M. DeMaria, and T. H. V. Haar, 2008: Tropical cyclone inner-core kinetic energy evolution. Mon. Wea. Rev., 136, 4882-4898.

Mallen, K. J., M. T. Montgomery, and B. Wang, 2005: Reexamining the near-core radial structure of the tropical cyclone primary circulation: Implications for vortex resiliency. $J$. Atmos. Sci., 62, 408-425.

Martinez, Y., G. Brunet, and M. K. Yau, 2010: On the dynamics of two-dimensional hurricane-like concentric rings vortex formation. J. Atmos. Sci., 67, 3253-3268.

Mellor, G. L., and T. Yamada, 1974: A hierarchy of turbulence closure models for planetary boundary layers. J. Atmos. Sci., 31, 1791-1806. 
Mlawer, E. J., S. J. Taubman, P. D. Brown, M. J. Iacono, and S. A. Clough, 1997: Radiative transfer for inhomogeneous atmosphere: RRTM, a validated correlated-k model for the longwave. J. Geophys. Res., 102 (D14), 16 663-16 682.

Montgomery, M. T., and R. J. Kallenbach, 1997: A theory for vortex Rossby-waves and its application to spiral bands and intensity changes in hurricanes. Q. J. Roy. Meteor. Soc., $123,435-465$.

Moon, Y., and D. S. Nolan, 2010: The dynamic response of the hurricane wind field to spiral rainband heating. J. Atmos. Sci., 67, 1779-1805.

Nakanishi, M. and H. Niino, 2004: An improved Mellor-Yamada Level-3 model with condensation physics: Its design and verification. Boundary-Layer Meteor., 112, 1-31.

Nolan D. S., J. A. Zhang, and D. P. Stern, 2009a: Evaluation of planetary boundary layer parameterizations in tropical cyclones by comparison of in-situ data and high-resolution simulations of Hurricane Isabel (2003). Part I: Initialization, maximum winds, and outer core boundary layer structure. Mon. Wea. Rev., 137, 3651-3674.

Nolan D. S., D. P. Stern, and J. A. Zhang, 2009b: Evaluation of planetary boundary layer parameterizations in tropical cyclones by comparison of in-situ data and high-resolution simulations of Hurricane Isabel (2003). Part II: Inner-core boundary layer and eyewall structure. Mon. Wea. Rev, 137, 3675-3698.

Nolan, D. S., 2011: Evaluating Environmental Favorableness for Tropical Cyclone Development with the Method of Point-Downscaling. Journal of Advances in Modeling Earth Systems, 3.

Nolan, D. S., and M. T. Montgomery, 2002: Nonhydrostatic, three-dimensional perturbations to balanced, hurricane-like vortices. Part I: Linearized formulation, stability, and evolution. J. Atmos. Sci., 59, 2989-3020.

Nolan, D. S., Y. Moon, and D. P. Stern, 2007: Tropical cyclone intensification from asymmetric convection: Energetics and efficiency. J. Atmos. Sci., 64, 3377-3405.

Nong, S., and K. Emanuel, 2003: A numerical study of the genesis of concentric eyewalls in hurricanes. Quart. J. Roy. Meteor. Soc., 129, 3323-3338.

Powell, M. D., P. J. Vickery, and T. A. Reinhold, 2003: Reduced drag coefficient for high wind speeds in tropical cyclones. Nature, 422, 279-283.

Reasor, P. D., M. T. Montgomery, F. D. Marks Jr., and J. F. Gamache, 2000: Lowwavenumber structure and evolution of the hurricane inner core observed by airborne dual-Doppler radar. Mon. Wea. Rev., 128, 1653-1680.

Rotunno, R., Y. Chen, W. Wang, C. Davis, J. Dudhia, G. J. Holland, 2009: Large-Eddy Simulation of an Idealized Tropical Cyclone. Bull. Amer. Meteor. Soc., 90, 1783-1788. 
Rozoff, C. M., D. S. Nolan, J. P. Kossin, F. Zhang, and J. Fang, 2012: The roles of the expanding wind field and inertial stability in tropical cyclone secondary eyewall formation. J. Atmos. Sci., 69, 2621-2643.

Rozoff, C. M., W. H. Schubert, and J. P. Kossin, 2008: Some dynamical aspects of tropical cyclone concentric eyewalls. Quart. J. Roy. Meteor. Soc., 134, 583-593.

Schubert W. H., M. T. Montgomery, R. K. Taft, T. A. Guinn, S. R. Fulton, J. P. Kossin, and J. P. Edwards. 1999: Polygonal eyewalls, asymmetric eye contraction, and potential vorticity mixing in hurricanes. J. Atmos. Sci., 56, 1197-1223.

Shamarock, W., J. B. Klemp, J. Dudhia, D. O. Gill, D. M. Barker, M. Duda, X. Y. Huang, W. Wang, and J. G. Powers, 2008: A description of the advanced research WRF version 3. NCAR technical note NCAR/TN/u2013475+ STR.

Shapiro, L. J., and H. E. Willoughby, 1982: The response of balanced hurricanes to local sources of heat and momentum. J. Atmos. Sci., 39, 378-394.

Smagorinsky, J. ,1963: General circulation experiments with the primitive equations: i. The basic experiment*. Mon. Wea. Rev.91, 99-164.

Smith R. K., M.T. Montgomery, H. Zhu, 2005: Buoyancy in tropical cyclones and other rapidly rotating vortices. Dyn. Atmos. Oceans 40: 189-208.

Stern, D. P., and D. S. Nolan, 2009: Reexamining the vertical structure of tangential winds in tropical cyclones: Observations and theory. J. Atmos. Sci., 66, 3579-3600.

Stikowski, M., J. P. Kossin, and C. M. Rozoff, 2011: Intensity and structure changes during hurricane eyewall replacement cycles. Mon. Wea. Rev., 139, 3829-3847.

Sun, Y. Q., Y. Jiang, B. Tan, F. Zhang, 2013: The Governing Dynamics of the Secondary Eyewall Formation of Typhoon Sinlaku (2008). J. Atmos. Sci., 70, 3818-3837.

Terwey, W. D., and M. T. Montgomery, 2008: Secondary eyewall formation in two idealized, full-physics modeled hurricanes. J. Geophys. Res., 113, D12112.

Thompson, G., R. M. Rasmussen, and K. Manning, 2004: Explicit forecasts of winter precipitation using an improved bulk microphysics scheme. Part I: Description and sensitivity analysis. Mon. Wea. Rev., 132, 519-542.

Wang, Y., 2009: How do outer spiral rainbands affect tropical cyclone structure and intensity? J. Atmos. Sci., 66, 1250-1273.

Willoughby H. E., and P. G. Black, 1996: Hurricane Andrew in Florida: dynamics of a disaster. Bull. Am. Meteorol. Soc., 77, 265-274.

Willoughby, H. E., 1978: A Possible Mechanism for the formation of hurricane rainbands. J. Atmos. Sci., 35, 838-848. 
Willoughby, H. E., 1979: Forced secondary circulations in hurricanes. J. Geophys. Res., 84, 3173-3183.

Willoughby, H. E., 1990: Temporal changes of the primary circulation in tropical cyclones. J. Atmos. Sci., 47, 242-264.

Willoughby, H. E., J. A. Clos, and M. G. Shoreibah, 1982: Concentric eyewalls, secondary wind maxima, and the evolution of the hurricane vortex. J. Atmos. Sci., 39, 395-411.

Wood, V. T., and L. W. White, 2011: A new parametric model of vortex tangential-wind profiles: Development, testing, and verification. J. Atmos. Sci., 68, 990-1006.

Wu, Y.-H. Huang, and G.-Y. Lien, 2012: Concentric eyewall formation in Typhoon Sinlaku (2008). Part I: Assimilation of T-PARC data based on the ensemble Kalman filter (EnKF). Mon. Wea. Rev., 140, 506-527.

Zhang, D. L., Y., Liu, and M. K. Yau, 2002: A multiscale numerical study of Hurricane Andrew (1992). Part V: Inner-core thermodynamics. Mon. Wea. Rev., 130, 2745-2763.

Zhou, X., and B. Wang, 2011: Mechanism of concentric eyewall replacement cycles and associated intensity change. J. Atmos. Sci., 68, 972-988.

Zhu P., M. Konstantinos, and Z. Zhu, 2013: Impact of subgrid-scale vertical turbulent mixing on eyewall asymmetric structures and mesovortices of hurricanes. Q. J. Roy. Meteor. Soc., doi: 10.1002/qj.2147. 
VITA

ZHENDUO ZHU

Born, Yongning, Ningxia, China PR

B.A., Atmospheric Sciences

Nanjing University

Nanjing, Jiangsu, China PR

2007

M.S., Atmospheric Sciences

Chinese Academy of Meteorological Sciences

Beijing, China PR

2007-2008

National Meteorological Center

Bejing, China PR

2011-2012

Teaching Assistant

Florida International University

Miami, Florida

2013-2014

Doctoral Candidate

Florida International University

Miami, Florida

\section{PUBLICATIONS AND PRESENTATIONS}

Zhu P., M. Konstantinos, and Z. Zhu, 2013: Impact of subgrid-scale vertical turbulent mixing on eyewall asymmetric structures and mesovortices of hurricanes. Q. J. Roy. M teor. Soc., doi: 10.1002/qj.2147.

Zhu, P., J. Dudhia, P. R. Field, K. Wapler, A. Fridlind, A. Varble, E. Ziper, J. Petch, M. Chen, Z.-D. Zhu, 2012: A limited area model (LAM) intercomparison study of a TWP ICE active monsoon mesoscale convective event. J. Geophys. Res., 117, D11208, 21 PP. doi:10.1029/2011JD016447.

Zhu, P., B. A. Albrecht, V. P. Ghate, and Z. Zhu, 2010: Multiple-scale simulations of stratocumulus clouds, J. Geophys. Res., 115, D23201, doi:10.1029/2010JD014400. 